\title{
Development of a Landing Period Indicator and the use of Signal Prediction to Improve Landing Methodologies of Autonomous Unmanned Aerial Vehicles on Maritime Vessels
}

\author{
by
}

\section{Shadi Abujoub}

A thesis submitted to the Faculty of Graduate and Postdoctoral Affairs in partial fulfillment of the requirements for the degree of

\author{
Master of Applied Science \\ in \\ Mechanical Engineering \\ Department of Mechanical and Aerospace Engineering \\ Carleton University \\ Ottawa, Ontario \\ (c) 2019 \\ Shadi Abujoub
}




\section{Abstract}

Unmanned aerial vehicles (UAVs) are becoming more prevalent in maritime operations. One of the key challenges to the safe operation of UAVs at sea is the relative motion that exists between the UAV and ship. The scope of this thesis is the creation and evaluation of a methodology for improving the overall landing performance for UAVs using signal prediction and a developed Landing Period Indicator (LPI). The research is conducted in a synthetic environment, where the test vehicle is a quad rotor UAV that is equipped with a Light Detection and Ranging (LIDAR) system to aerially detect ship motion. The observed ship motion is forecasted using signal prediction which identifies and notifies the UAV of potential landing opportunities. The Signal Prediction Algorithm (SPA) is also used for Active Heave Compensation (AHC) to facilitate the UAV in maintaining a safe low hover position above the ship deck. Further, an algorithm is developed to use the AHC system to plan trajectories that land the UAV with a specified impact velocity. The development of the LPI system is presented and its performance as a standalone and supplemental system is evaluated. ShipMo3D was used to generate 105 sets of ship motion in sea states 2-6. The results in this thesis indicate that the developed landing methodologies can improve the landing performance of ocean going helicopters. For the 105 sets of ship motion, using a combination of the SPA, AHC, and the LPI improved landing performance by $25 \%$ in two separate test groups. Moreover, the results indicate that with further tuning of the SPA, the likelihood of a safe landing can be further improved. 


\section{Contents}

Abstract $\quad$ i

List of Figures $\quad$ vi

List of Tables viii

List of Abbreviations \& Symbols $\quad$ ix

1 Introduction 1

2 Background and Literature Review $\quad 6$

2.1 Helicopter Sea Keeping . . . . . . . . . . . . . . . . . . . . . . 7

2.2 Ocean Waves, Wind and Ship Interaction . . . . . . . . . . . . . . . 10

2.3 Unmanned Landing Technology . . . . . . . . . . . . . . . . . . 13

2.4 Ship Motion Prediction . . . . . . . . . . . . . . . . . . . . 15

2.5 Quad Rotor . . . . . . . . . . . . . . . . . . . 17

2.5.1 Quad Rotor Control ................. 18

2.6 Modelling Methods . . . . . . . . . . . . . . . . . . . . 19

2.7 Summary . . . . . . . . . . . . . . . . . . 24

3 Model \& Synthetic Environment 25

3.1 Model Architecture . . . . . . . . . . . . . . . . . . . . . . . 25

3.2 Motor Modelling \& Propeller Theory . . . . . . . . . . . . . . . 28

3.2.1 Motor Modelling . . . . . . . . . . . . . . . . . 29

3.2.2 Propeller Theory . . . . . . . . . . . . . . . . . . 30

3.2 .3 Ground Effect . . . . . . . . . . . . . . . . . . . 36

3.3 UAV Aerodynamics . . . . . . . . . . . . . . . . . . . . 36

3.3.1 UAV Form Drag . . . . . . . . . . . . . . . . . . 37

3.3 .2 UAV Skin Drag . . . . . . . . . . . . . . . . . . . . . . 41

3.4 Wind Modelling . . . . . . . . . . . . . . . . . . . . . 44

3.4.1 Wind Gusts . . . . . . . . . . . . . . . . . 44

3.4.2 Atmospheric Turbulence . . . . . . . . . . . . . . . 46

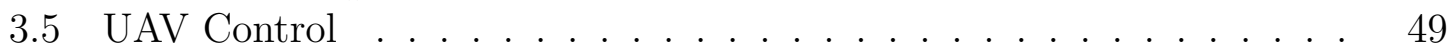

3.5.1 Altitude Control .................. 50

3.5.2 Yaw Control . . . . . . . . . . . . . . . . 52

3.5.3 Roll and Pitch Control . . . . . . . . . . . . . . 54 
3.5.4 Trajectory Tracking Control . . . . . . . . . . . . . . 56

3.6 Trajectory Tracking Results . . . . . . . . . . . . . . . . . . . . . 59

3.7 Ship Motion . . . . . . . . . . . . . . . . . . . . . 64

3.8 LIDAR and Ship Pose Detection . . . . . . . . . . . . . . . . 68

3.8.1 LIDAR Emulation . . . . . . . . . . . . . . . . . . . . . 69

3.8.2 Ship Pose Detection _... . . . . . . . . . . . 71

3.8.3 LIDAR Noise \& Filtering . . . . . . . . . . . . . . . . 72

3.9 Summary . . . . . . . . . . . . . . . . . . . . . . . . . . . . . 78

4 Flight Control For Autonomous Landing at Sea 80

4.1 Signal Prediction Algorithm . . . . . . . . . . . . . . . . 81

4.1 .1 SPA Derivation . . . . . . . . . . . . . . . . . 82

4.1.2 SPA Prediction Horizon for Landing . . . . . . . . . . . 85

4.1 .3 Go-NoGo Command Latching . . . . . . . . . . . . . . . 87

4.2 Active Heave Compensation using Signal Prediction . . . . . . . 87

4.2.1 Active Heave Compensation - Low Hover . . . . . . . . . . . 88

4.2.2 Active Heave Compensation - Landing . . . . . . . . . . 88

4.2.3 Performance of AHC for landing . . . . . . . . . . . . . 92

4.3 Landing Period Indicator . . . . . . . . . . . . . . . . . . . . 93

4.3.1 Proposed Landing Period Indicator . . . . . . . . . . . . . 94

4.3.2 Comparison of LPI and SPA . . . . . . . . . . . . . . 101

4.4 Flight Controller . . . . . . . . . . . . . . . . . . . . . . . . 103

4.5 Summary . . . . . . . . . . . . . . . 106

5 Results 107

5.1 Summary of Results . . . . . . . . . . . . . . . . . . . . . . . . 109

5.2 Results - Low Hover, $2.5 \mathrm{~m}$. . . . . . . . . . . . . . . . . . 112

5.3 Results - Low Hover, $5 \mathrm{~m} \ldots \ldots \ldots \ldots$

5.4 Discussion . . . . . . . . . . . . . . . . . . . . . . . . . . . 117

6 Conclusions and Future Work 119

6.1 Future Work . . . . . . . . . . . . . . . . . . . . . . . . . . . . 121

$\begin{array}{ll}\text { References } & 123\end{array}$

A Full Results - Low Hover, $2.5 \mathrm{~m} \quad 133$

B Full Results - Low Hover, 5 m 141 


\section{List of Figures}

1.1 UAV and ship body coordinate systems with respect to the global coordinate system. . . . . . . . . . . . . . . 2

2.1 Bell Helicopter's 20 year, world-wide, crash history (1985-2004) [12] . 7

2.2 USCG general launch and recovery wind and ship motion envelope for helicopter landing $[5] \ldots \ldots$. . . . . . . . . . . . . . . . . . 9

2.3 Information structure of the Signal Prediction Algorithm [9]. . . . . . 17

2.4 Typical quad rotor configurations. + (left),$\times$ (right) . . . . . . . 18

2.5 Simulated wind flow over a ship deck using CFD [52] . . . . . . . . . 22

3.1 Front view dimensional drawing of UAV with units displayed in $\mathrm{m}$ and

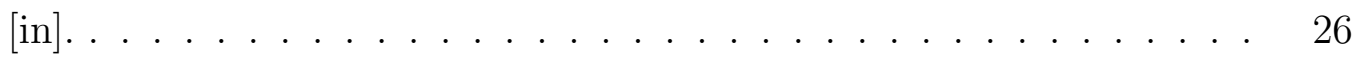

3.2 Model information flow within the simulator. . . . . . . . . . . . 27

3.3 Subsystems of the quad rotor UAV model. . . . . . . . . . . . . . . 28

3.4 PWM controlled motor with torque feedback in Simscape. . . . . . . 29

3.5 Static thrust and error comparison between measured data, theoretical propeller thrust equation and G.S corrected thrust equation. . . . . . 32

3.6 Propeller performance characteristics for a $10 \times 5$ in propeller. . . . . . 34

3.7 UIUC propeller $10 \times 5$ in propeller thrust and torque characteristics. . 35

3.8 Static thrust comparison between measured data, theoretical calculations and empirically driven equations. . . . . . . . . . . . 35

3.9 (a) $2 \mathrm{D}$ projection of UAV during accent with no cross winds. (b) 2D projection of pitched UAV travelling in head wind. (c) Rolled and pitched UAV traveling with equal x-y velocity components. . . . . . . 40 
3.10 Coefficient of lift Vs. angle of attack for a flat plate. . . . . . . . . .

3.11 1-cosine shape wind gust with a peak velocity reached at a fetch length of $10 \mathrm{~km} . \ldots \ldots \ldots \ldots \ldots \ldots$

3.12 Atmospheric turbulence and wind gusts a UAV encounters when flying in sea state 6 with a wind direction of $60^{\circ}$. . . . . . . . . . . . . 49

3.13 PID controller for UAV altitude. . . . . . . . . . . . . . . 51

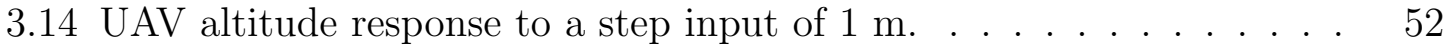

3.15 P-PI control strategy for UAV yaw. . . . . . . . . . . . . . . . 54

3.16 Yaw P-PI controller vs. PID controller disturbance rejection to a load applied on the UAV arm, resulting in a 0.7 Nm moment about the CG. 54

3.17 Cascade PI-PID control strategy for UAV roll and pitch controllers. . 55

3.18 Outer and inner-loop response of a UAV roll controller subjected to a $15^{\circ}$ step input. . . . . . . . . . . . . . . . 56

3.19 Control strategy for the UAV trajectory tracking controllers. . . . . . 57

3.20 Trajectory tracking results for a $50 \mathrm{~m}$ step input. . . . . . . . . . . . 58

3.21 (a) Satisfactory UAV trajectory tracking of a spiral in sea state 4 winds with a wind direction of $90^{\circ}$. (b) Unsatisfactory trajectory tracking results of a UAV following a spiral in sea state 5 winds with a wind direction of $0^{\circ}$ (c) UAV unable to track spiral trajectory in sea state 5 winds blowing at $30^{\circ} \ldots \ldots$. . . . . . . . . . . . . . . 60

3.22 Starting position of UAV during simulations . . . . . . . . . . . 62

3.23 Tracking error between UAV and ship landing zone while maintaining a low hover of $5 \mathrm{~m} . \ldots \ldots \ldots$. . . . . . . . . . . . 63

3.24 (a) $30^{\circ}$ ship heading,(b) $120^{\circ}$ ship heading. . . . . . . . . . . . 65

3.25 Roll and pitch angles of a ship travelling in sea state 3 with a $15^{\circ}$ heading and corresponding Go NoGo states. . . . . . . . . . . . . 66

3.26 Ship heading angle influence on ship motion during sea state $4 . \quad$. . . 67

3.27 Schematic of UAV and the notation used to derive the simulated LIDAR outputs. . . . . . . . . . . . . . . . . . 69

3.28 Ship pose detection illustrated notation. . . . . . . . . . . . . 72 
3.29 LIDAR spacing influence on gathered ship motion RMSE and SNR. .

3.30 LIDAR noise Filtering RMSE results for improving LIDAR based ship pose detection. . . . . . . . . . . . . . . . . . . . 77

3.31 LIDAR noise Filtering SNR results for improving LIDAR based ship pose detection. . . . . . . . . . . . . . . . . . . 77

4.1 Information structure of the Signal Prediction Algorithm [9] . . . . . 84

4.2 UAV landing protocol using the SPA. . . . . . . . . . . . . 86

4.3 Using the signal prediction algorithm to develop landing trajectories for the UAV. . . . . . . . . . . . . . . . . . . . . . . . . . . . 89

4.4 Predicted impact velocities from the AHC landing trajectories. . . . . 90

4.5 Determining ship heave motion in the global coordinate frame. . . . . 92

4.6 120 second evaluation of ship motion for Go periods lasting longer than 5 seconds. . . . . . . . . . . . . . . . . . . 95

4.7 Energy index of ship roll pitch and heave. . . . . . . . . . . . . 96

4.8 Normalized energy index of ship roll pitch and heave. . . . . . . . . . 97

4.9 Un-penalized landing period indicator. . . . . . . . . . . . . . . 98

4.10 Landing period indicator with penalty function. . . . . . . . . 100

4.11 Simulink block structure for real time implementation of LPI. . . . . 101

4.12 State machine flight controller . . . . . . . . . . . . . . 106 


\section{List of Tables}

2.1 Douglas sea state [25] \& Beaufort wind scale [26]. . . . . . . . . . . . 13

3.1 Modeled PWM, H-bridge, and motor parameters. . . . . . . . . . . . 30

3.2 Operational limits of UAV. . . . . . . . . . . . . . . . 43

3.3 Expected wind velocities and fetch lengths for sea states 2-6. . . . . . 45

3.4 PID gains used for UAV control and trajectory tracking. . . . . . . . 58

3.5 Spiral trajectory tracking results. . . . . . . . . . . . . . . 61

3.6 UAV ship tracking error for sea state 6 ship motion with winds of $10 \mathrm{~m} / \mathrm{s} .63$

3.7 Ship motion cases created in ShipMo3D used in UAV-ship simulator. 64

3.8 Average Go time sustained for $5 s$ or more vs. sea state for simulated ship motion (21 sets of ship motion data per sea state). . . . . . . 68

3.9 LIDAR filtering combinations. Trial numbers correlate to x-axis of Figures 3.30. \& 3.31 . . . . . . . . . . . . . . . . . . . . 77

4.1 Impact velocity comparison between using the standard landing methods and using the active heave compensation algorithm for headings of $0^{\circ}, 30^{\circ}, 60^{\circ}$, and $90^{\circ}$ in sea states 4,5 , and 6 . . . . . . . . . 93

4.2 Efficiencies and state changes for LPI system and SPA . . . . . . . 103

5.1 Ship Landing Test Cases . . . . . . . . . . . . . . . . . . . . . . . 108

5.2 Ship Motion Combinations . . . . . . . . . . . . . . . . . . 108

5.3 Executive summary of 2.5 m low hover results. . . . . . . . . . . . . . 110

5.4 Executive summary of $5 \mathrm{~m}$ low hover results. . . . . . . . . . . . . . . 112

5.5 Summary of $2.5 \mathrm{~m}$ low hover results. . . . . . . . . . . . . . . . . 113 
5.6 Average land time, simulation time, impact velocity and land angle for the $2.5 \mathrm{~m}$ low hover test cases. . . . . . . . . . . . . . . . . . 114

5.7 Summary of $5 \mathrm{~m}$ low hover results. . . . . . . . . . . . . 116

5.8 Average land time, simulation time, impact velocity and land angle for the $5 \mathrm{~m}$ low hover test cases. . . . . . . . . . . . . . . 117

A.1 Unregulated $2.5 \mathrm{~m}$ low hover full results. . . . . . . . . . . . . 134

A.2 SPA $2.5 \mathrm{~m}$ low hover full results. . . . . . . . . . . . . 135

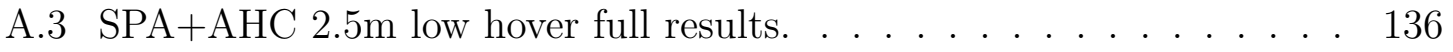

A.4 LPI 2.5m low hover full results. . . . . . . . . . . . . . . . 137

A.5 LPI+SPA 2.5m low hover full results. . . . . . . . . . . 138

A.6 LPI+SPA+AHC $2.5 \mathrm{~m}$ low hover full results. . . . . . . . . . . . . 139

A.7 LPI+AHC $2.5 \mathrm{~m}$ low hover full results. . . . . . . . . . . . . 140

B.1 Unregulated 5m low hover full results. . . . . . . . . . . . . . 142

B.2 SPA $5 \mathrm{~m}$ low hover full results. . . . . . . . . . . . . . . . . 143

B.3 SPA+AHC 5m low hover full results. . . . . . . . . . . . . 144

B.4 LPI 5m low hover full results. . . . . . . . . . . . . . . . 145

B.5 LPI+SPA 5m low hover full results. . . . . . . . . . . . . 146

B.6 LPI+SPA+AHC 5m low hover full results. . . . . . . . . . . . 147

B.7 LPI+AHC 5m low hover full results. . . . . . . . . . . . . 148 


\title{
List of Abbreviations \& Symbols
}

\author{
List Of Abbreviations
}

$\begin{array}{ll}\text { AHC } & \text { Active Heave Compensation } \\ \text { AR } & \text { Auto Regressive } \\ \text { ARMA } & \text { Auto Regressive Moving Average } \\ \text { AW } & \text { Air Worthiness } \\ \text { CAD } & \text { Computer Aided Design } \\ \text { CFD } & \text { Computational Fluid Dynamics } \\ \text { EI } & \text { Energy Index } \\ \text { EMA } & \text { Exponential Moving Average } \\ \text { FEM } & \text { Finite Element Modelling } \\ \text { FFT } & \text { Fast Fourier Transform } \\ \text { G.S. } & \text { Gabriel Staples } \\ \text { GPS } & \text { Global Positioning System } \\ \text { JONSWAP } & \text { Joint North Sea Wave Project } \\ \text { KF } & \text { Kalman Filter } \\ \text { LH } & \text { Low Hover } \\ \text { LIDAR } & \text { Light Detection and Ranging } \\ \text { LPI } & \text { Landing Period Indicator } \\ \text { LQR } & \text { Linear Quadratic Regulator } \\ \text { LA } & \text { Landing Zone } \\ \text { MAing Average }\end{array}$




$\begin{array}{ll}\text { MCA } & \text { Minor Component Analysis } \\ \text { MPC } & \text { Model Predictive Control } \\ \text { NN } & \text { Neural Network } \\ \text { PDE } & \text { Partial Differential Equation } \\ \text { PID } & \text { Proportional-Integral-Derivative } \\ \text { PWM } & \text { Pulse Width Modulation } \\ \text { QR } & \text { Quad Rotor } \\ \text { RAST } & \text { Recovery, Assist and Traverse } \\ \text { SHOL } & \text { Ship Helicopter Operating Limit } \\ \text { SPA } & \text { Signal Prediction Algorithm } \\ \text { TAS } & \text { True Air Speed } \\ \text { UAV } & \text { Unmanned Aerial Vehicle } \\ \text { VTOL } & \text { Vertical Take Off and Landing } \\ \text { Z-N } & \text { Ziegler-Nicholas }\end{array}$

\section{Greek Symbols}

$\begin{array}{ll}\alpha & \text { Angle of attack } \\ \bar{x}_{k} & \text { Filtered averaged output } \\ \epsilon_{k} & \text { Error covariance } \\ \eta_{m} & \text { Motor efficiency } \\ \gamma & \text { Wave peak } \\ \kappa & \text { Correction factor } \\ \Lambda & \text { Discrete system matrix } \\ \lambda_{k} & \text { Forgetting factor } \\ \omega & \text { Angular frequency } \\ \Omega_{m} & \text { Motor angular velocity } \\ \Phi & \text { X rotation angle } \\ \Phi_{i} & \text { Phase of signal }\end{array}$




$\begin{array}{ll}\Phi_{u, v, w} & \text { Spectral energy } \\ \Psi & \text { Z rotation angle } \\ \rho & \text { Air density } \\ \sigma & \text { Variance } \\ \sigma_{u, v, w} & \text { Turbulence intensity in } \mathrm{u}, \mathrm{v}, \mathrm{w} \text { direction } \\ \Theta & \text { Y rotation angle } \\ v & \text { Kinematic viscosity of air } \\ n_{s} & \text { Number of samples } \\ w_{k} & \text { Weighting factor }\end{array}$

\section{Roman Symbols}

$\begin{array}{ll}\dot{m} & \text { Mass flow rate } \\ \dot{x} & \text { Observer model } \\ \hat{n}_{L} & \text { LIDAR directional Vector } \\ \hat{V}_{e} & \text { Estimated exit velocity } \\ \hat{V}_{T A S} & \text { True airspeed direction vector } \\ \hat{x}_{k} & \text { State estimate } \\ \mathrm{E} & \text { Matrix of Gains } \\ \vec{L}_{i} & \text { LIDAR Vector } \\ \vec{n}_{L} & \text { LIDAR Normal Vector } \\ \vec{P}_{i} & \text { LIDAR measurement vector to ship } \\ \vec{S}_{i} & \text { Vector to point on ship } \\ \vec{V}_{T A S} & \text { UAV true airspeed } \\ \vec{V}_{U A V} & \text { UAV ground airspeed } \\ \vec{V}_{W i n d} & \text { Wind speed } \\ \widetilde{X}_{w} & \text { Fully developed fetch length } \\ A_{i} & \text { Amplitude of signal } \\ A_{p} & \text { Propeller area } \\ & \end{array}$




\begin{tabular}{|c|c|}
\hline$A_{2 D}$ & Projected area \\
\hline$A_{w e t}$ & Wetted surface area \\
\hline$B$ & Denotes body frame \\
\hline$C$ & System output Matrix \\
\hline$C_{D}$ & Coefficient of drag \\
\hline$C_{f}$ & Coefficient of friction \\
\hline$C_{L}$ & Coefficient of lift \\
\hline$C_{P}$ & Coefficient of power \\
\hline$C_{T}$ & Coefficient of thrust \\
\hline$d_{p}$ & Propeller diametre \\
\hline$F_{D}$ & Drag Force \\
\hline$f_{i}$ & Frequency of signal \\
\hline$F_{L}$ & Lift Force \\
\hline$f_{s}$ & Sampling frequency \\
\hline$g$ & Gravitational acceleration \\
\hline$G_{i}$ & Weighting gain \\
\hline$H(s)$ & Continuous Transfer function \\
\hline$H_{i}$ & LIDAR magnitude \\
\hline$H_{k}$ & Observation Model \\
\hline$H_{1 / 3}$ & Significant wave height \\
\hline$J$ & Advance ratio \\
\hline$K_{d}$ & Derivative gain \\
\hline$K_{e}$ & Kinetic energy \\
\hline$K_{i}$ & Integral gain \\
\hline$K_{k}$ & Kalman Gain \\
\hline$K_{p}$ & proportional gain \\
\hline$K_{u}$ & Ultimate gain \\
\hline
\end{tabular}




\begin{tabular}{|c|c|}
\hline$K_{v}$ & Motor velocity constant \\
\hline$l$ & Length \\
\hline$L_{d}$ & Vertical component of LIDAR measurement \\
\hline$L P I$ & Landing Period Indicator value \\
\hline$N$ & Number of modes detected by FFT \\
\hline$N_{i}$ & Normalizing coefficient \\
\hline$p$ & Ship roll angle \\
\hline$p_{i}$ & LIDAR measurement point on ship \\
\hline$P_{p}$ & Propeller pitch \\
\hline$P_{L P I}$ & Penalty value \\
\hline$p_{R M S}$ & Root mean square of roll \\
\hline$Q$ & Process noise covariance \\
\hline$q$ & Ship pitch angle \\
\hline$Q_{P}$ & Propeller reaction torque \\
\hline$q_{R M S}$ & Root mean square of pitch \\
\hline$R$ & Measurement noise covariance \\
\hline$r$ & Enhancement factor \\
\hline$R_{e}$ & Reynolds number \\
\hline$r_{p}$ & Propeller radius \\
\hline$R_{x}$ & Rotation about $\mathrm{x}$ axis \\
\hline$R_{y}$ & Rotation about y axis \\
\hline$R_{z}$ & Rotation about $\mathrm{z}$ axis \\
\hline$R_{F F T}$ & FFT resolution \\
\hline$s_{i}$ & Point on ship \\
\hline$T_{d}$ & Derivative gain \\
\hline$T_{p}$ & Thrust produced by propeller \\
\hline$T_{\text {Pred }}$ & Prediction horizon \\
\hline
\end{tabular}




$\begin{array}{ll}T R & \text { Thrust ratio } \\ u & \text { Longitudinal component } \\ U_{19.5} & \text { Wind velocity at } 19.5 \mathrm{ft} \\ U A V_{x y z} & \text { UAV point cloud coordinates } \\ v & \text { Lateral component } \\ V_{e} & \text { Exit velocity } \\ V_{L} & \text { UAV landing velocity } \\ V_{m} & \text { Voltage to motor } \\ V_{o} & \text { Inflow velocity } \\ v_{t} & \text { Static offset } \\ V_{g u s t} & \text { Wind gust velocity } \\ V_{L i} & \text { Landing velocity } \\ w & \text { Ship Heave position } \\ w & \text { Vertical component } \\ x_{k} & \text { model state } \\ x_{g u s t M a x} & \text { Distance into wind field to reach maximum wind velocity } \\ x_{g u s t} & \text { Distance travelled into wind gust field } \\ z_{w} & \text { Current measurement signal } \\ & \text { Fetch length } \\ & \text { Dito }\end{array}$




\section{Chapter 1}

\section{Introduction}

Rotorcraft are used for widespread civilian and military purposes such as surveillance [1], oceanographic data collection [2], wildlife monitoring [3] and search and rescue [4]. The availability of remote and autonomous systems is prompting the transition from previously manned missions to unmanned. Typical oceanographic flight missions for Unmanned Aerial Vehicles (UAVs) include coastal and oceanographic surveying, ice cap observation and oil spill monitoring. Data procurement packages such as cameras and ranging devices are commonly outfitted to rotorcraft to aid with research operations as well as to assist with complex flight manoeuvres.

Due to the limited range of small rotorcraft, and the isolated nature of oceanographic missions, it is often necessary to recover the UAV on the deck of a marine vessel (ship deck). The most common recovery method for rotary vehicles is a vertical landing onto the ship deck, however, ship deck landings are a complicated manoeuvre for any rotorcraft. Figure 1.1 illustrates the relative motion which exists between the two bodies, and highlights the independent body frames with respect to the global frame. The UAV and ship move independently of one another, during Vertical Take Off and Landing (VTOL) the UAV attempts to remain in-line with Landing Zone (LZ), indicated by the yellow circle with $H$ in the centre. Safety guidelines for 

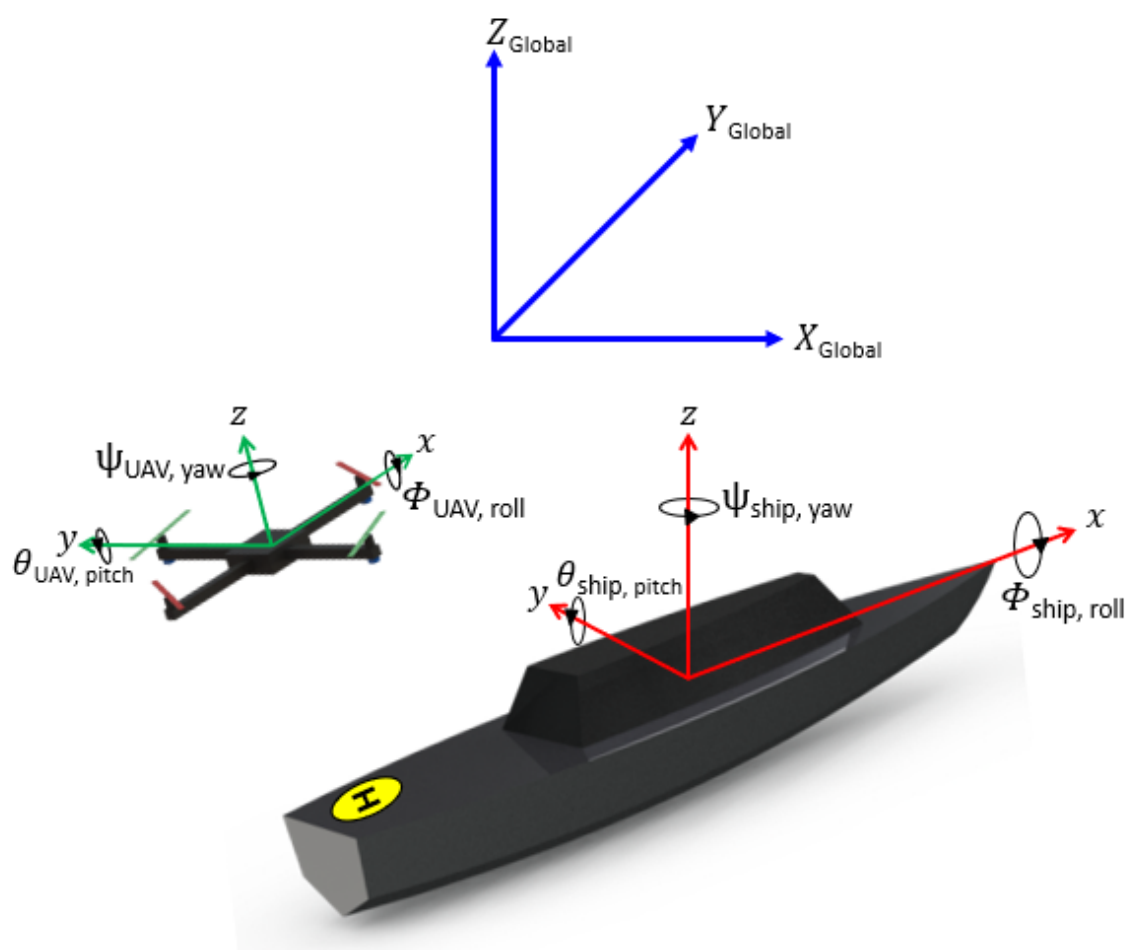

Figure 1.1: UAV and ship body coordinate systems with respect to the global coordinate system.

shipboard UAV landing operations prescribe threshold ship pitch and roll limits for landing [5]. These threshold values are indicators of when it is or is not safe to land.

Conventional VTOL relies on a human pilot's prior experience and intuition to predict and compensate for the relative motion between the two bodies, and accommodate for future ship displacements. However, on autonomous UAV's the challenges associated with landing safely increase. Autonomous UAV's must be able to decipher when to begin or abort a landing, as well as perform all other flight critical operations. Ships capable of accommodating VTOL vehicles may employ secondary hardware such as positioning beacons, transmitters and receivers to facilitate UAVs with landing [6]. Yet, the necessity for secondary hardware limits the cross-platform capability between UAVs and ally ships. Thus, the need arises for a UAV capable of autonomous VTOL with minimal secondary deck hardware or communication with 
the mating ship. Further, a platform is required to enable testing of various landing scenarios and methodologies. The first outcome of this research is to develop a simulator model includes:

- A quad rotor model capable of autonomous flight, ship tracking, and landing

- A flight environment that emulates an oceanic environment

- Baseline metrics for the success of the model simulations

Due to the stochastic nature of ship motion, there will be inherent uncertainty in predicting and delineating ship movement. This uncertainty adds increased strain on the already complex problem of landing a rotorcraft. However, if future ship motion were known, the landing performance of UAVs at sea could be improved. Signal prediction independent of vessel type is currently used for Active Heave Compensation (AHC) on ships utilizing towing winches, allowing the system to actively pay-out or reel-in line to the towed object to attenuate the transmission of the ship's motion to the submerged equipment [7]. It is hypothesized that the same Signal Prediction Algorithm (SPA) used by Woodacre [7] and improved upon by McPhee $[8,9]$ may be used on-board a UAV to facilitate landing. The second outcome of this thesis is the methodology for landing the UAV on a dynamic platform using the same signal prediction algorithm previously improved upon by McPhee. The objective is investigated by using the SPA to search for periods where the ship motion is within specified safe thresholds to land. The objective is further expanded to use the SPA to improve landing performance by compensating for the relative vertical displacement between the UAV and the ship. The developed system uses the SPA to predict future ship heave positions and preemptively adjusts the UAV's position to compensate for the expected ship movement. The Active Heave Compensation (AHC) system is also used to plan trajectories that land the UAV with a specified relative impact velocity. Initial testing of the AHC system is conducted to analyze its effectiveness. 
This research led to the development of a Landing Period Indicator (LPI) that was found to improve landing performance without the use of signal prediction. The LPI self-tunes its system parameters based off the observed ship motion recorded while in a high hover above the ship deck. The LPI attempts to identify the energy and rate of change of energy of the ship from the data collected during tuning and from the current ship motion. The LPI system will attempt to land the UAV when the ship energy is low.

A Proof-of-Concept $(\mathrm{PoC})$ study is performed to evaluate the landing performance of the UAV when landing based off the current ship motion and when using either the SPA, LPI, or AHC. The study assess the UAV's ability to land within specified roll and pitch thresholds with an impact velocity less than $1 \mathrm{~m} / \mathrm{s}$. The PoC study also analyzes the effects of combining the landing methodologies together and highlights the effectiveness of using the SPA to compensate for heave motion. The final outcome of this thesis is to analyze the effectiveness of the developed systems and highlight their respective operating bounds.

The key contributions of the research shown in this thesis are:

1. The development of an active heave compensation system which facilitates the UAV in:

- Maintaining a safe low hover position above the ship deck

- Landing with a specified goal impact velocity

2. The formulation of a landing period indicator to improve UAV landing performance without the use of signal prediction

3. A portion of this research has been presented and published in the proceedings of the IEEE Oceans 18 Conference [9]

There are six chapters in this thesis. Chapter 2 reviews existing literature pertaining to helicopter-ship operations, including: wave modelling, ship motion prediction, 
current landing technology, the UAV platform used in this thesis, and simulation modelling methods. Chapter 3 describes the methodology used to develop a simulator for autonomously landing a UAV on a ship. In Chapter 4, the various strategies used for improving the landing performance of UAVs is introduced, and the state-machine flight control is described. Chapter 5 highlights the results of using the developed systems to land on a maritime vessel in sea states 2 through 6 . Finally, the concluding remarks are given in Chapter 6. 


\section{Chapter 2}

\section{Background and Literature Review}

The success of modern day helicopter aviation may partially be attributed the Second World War. That is, there is correlation between wars and cash flow towards military ingenuity. The dawn of the Second World War accelerated helicopter research and development, casting a light on the future of maritime-helicopter operations. For example, the German Focke-Achgelias Drache, a twin three-bladed rotor helicopter was designed to be used for anti-submarine warfare, reconnaissance, search and rescue and transportation of cargo [10]. Further, the Germans developed the first helicopters primarily geared toward maritime operations, the Flettner Fl 265 and Fl 282. The Flettner's concentric counter-rotating propellers, the first of its kind, allowed for an air frame small enough for ship deck launch and recovery [11]. Thus began the rapid evolution from the, relatively speaking, primitive 20th century helicopters to today's generation of sophisticated rotorcraft.

Today's era of helicopters are able to sustain longer flights in harsher environments, with most capable of ship deck launch and recovery. Due to perpetually advancing avionics and design, helicopter crashes are on a steady decline from just 30 years ago [12]. Figure 2.1 is a 20 year history spanning from 1985-2004 of Bell Helicopter crashes. The crashes are categorized into a general all cause group and 
two Air Worthiness (AW) failures groups. AW relates to either flight system or mechanical failures. The AW failures were split into two categories, engine AW and non-engine AW related crashes. There is a steady decline in all 3 groups, correlating to a significant increase in safety considering that in 2002 there were over 2.1 million helicopter aviation flight hours [13]. Further, from 2007-2017 it was found that 33\% off all helicopter crashes in Canada occurred during landing, where the helicopter either collided with an object, had a hard landing, collided with terrain or lost control [14]. The dangers of landing are further exacerbated while a helicopter attempts to land on a ship due to the confided space of the ship deck, poor visibility from oceanic weather, and the relative motion between the helicopter and ship.

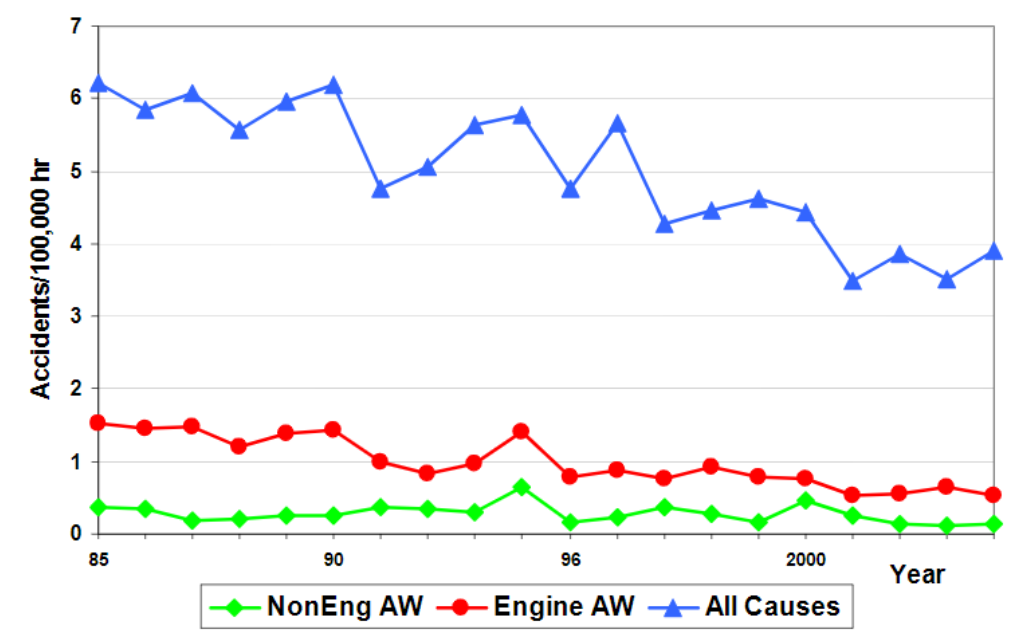

Figure 2.1: Bell Helicopter's 20 year, world-wide, crash history (1985-2004) [12]

\section{$2.1 \quad$ Helicopter Sea Keeping}

Even with the advancement in helicopter aviation, helicopter operation is far from trivial. As mentioned, one particularly challenging operating environment for helicopters is VTOL on a ship. The relative motion between a helicopter and a ship is the primary challenge, which is further intensified by oceanic wind loading and airflow hazards from rotor vortices and superstructure wake turbulence [15]. De- 
creased visibility from weather and night time operation further complicate ship deck VTOL. To increase the probability of safe deck landings there are stringent landing protocols as well as deck and crew requirements [16]. An example of deck requirements are visual landing aids, deck flood lighting during night operation, and nylon guard webbing [16]. Personnel are essential to successful helicopter landings; officers, ground crew and safety divers facilitate VTOL [16]. To further ensure safe landings, Ship Helicopter Operating Limits (SHOL) are used to outline operating bounds between helicopters and mating ships. SHOLs are expressed in terms of relative wind direction and velocities, and ship roll and pitch threshold limits. Figure 2.2 is an example of the United States Coast Guard SHOL for general helicopter ship pairings, however more specific SHOL's pertaining to individual ship-helicopter pairings are available [5]. The safe motion thresholds for landing are indicated at the bottom of the figure; in this instance, it is deemed safe to land a helicopter if the absolute value of pitch and roll are less than $2^{\circ}$ and $4^{\circ}$, respectively. The relative velocity direction between the helicopter and ship is outlined on the boundary of the SHOL, and on the centre axis is the maximum allowable relative wind velocity to attempt a landing. In order to be cleared for landing the ship motion must be less than the threshold limits, and the relative wind velocity and direction must be within the SHOL envelope. For example, if the relative wind velocity direction is $20^{\circ}$ and is blowing at less than 15 knots $(\mathrm{kn})$ the helicopter would be cleared to land if the ship's motion is less than the threshold values. However, if the relative wind direction is $45^{\circ}$ at $15 \mathrm{kn}$ the helicopter is not cleared to land regardless of the ship motion. With proper equipment, staff, and abiding by the SHOL, the probability of a safe helicopter landing increases.

Circumstances exist in which a helicopter may not be able to obey a SHOL for landing, such as during prolonged storms or rough seas. If adverse weather conditions persist, a pilot may decide to land despite the established safe landing guidelines. To mitigate the risks while landing during unfavorable wind and ship motion conditions, 


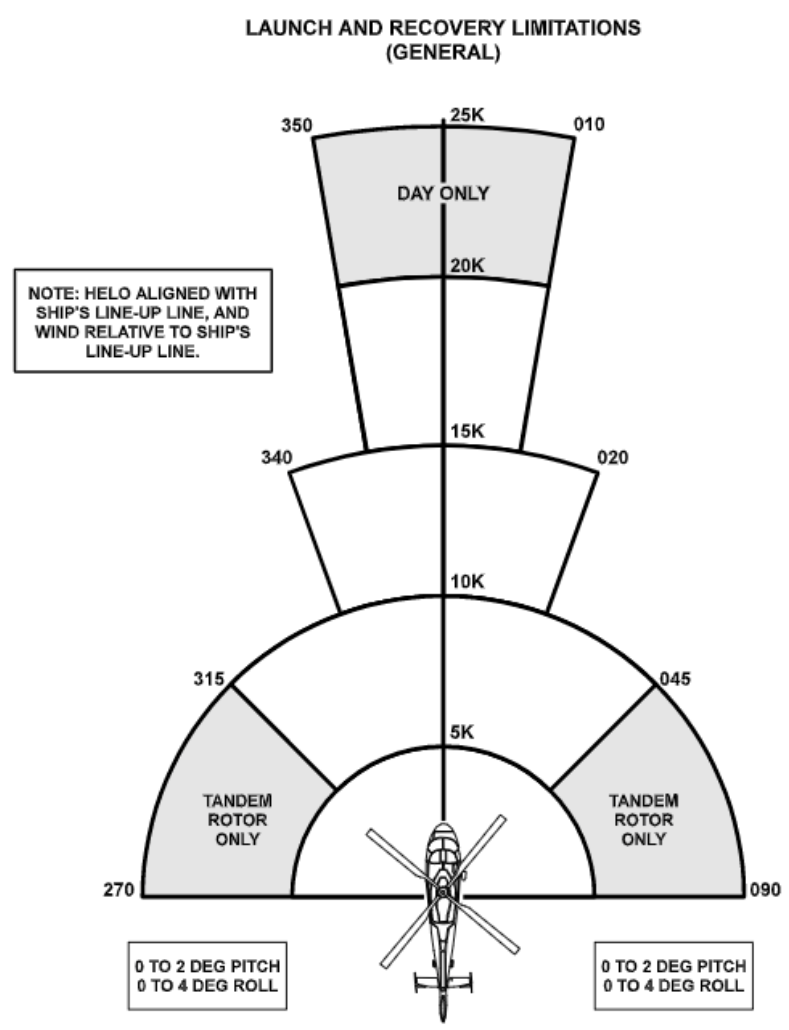

Figure 2.2: USCG general launch and recovery wind and ship motion envelope for helicopter landing [5].

systems have been developed to assist helicopters mate with ships. Curtiss-Wright, a Canadian company which specializes in Recovery, Assist, Secure and Traverse (RAST) systems, developed a ship deck mounted system, TC-Assist, that latches onto a probe hanging from a helicopter to aid pilots in landing. Their system encompasses a vision system to guide the recovery hardware beneath the probe which subsequently latches onto the probe [17]. Other RAST systems, such as the Heligrid [18], install a harpoon onto helicopters and a grid plate onto the mating ship. When landing, the harpoon claw is open and extended beneath the helicopter. The pilot manoeuvres the helicopter to position the claw into the grid plate and subsequently latches on, thus locking the helicopter to the ship. Both of the aforementioned RAST systems rely on pilot skills to safely reach the deck mounted hardware. To improve landing performance in extreme ship motion conditions, Curtiss-Wright developed the Mark 6. 
The Mark 6 uses a deck winch-cable system to reel the helicopter to the deck. RAST systems are effective for landing helicopters, however, the reliance on deck mounted hardware, their high cost, and the need for additional operators in the loop limits their widespread use. Researchers are continually searching for methods to improve helicopter-ship interactions. In order to develop landing methodologies and equipment to facilitate inter-operability of helicopter-ship interfacing, the correspondence between oceanic conditions, ship motion, and SHOLs must be examined.

\subsection{Ocean Waves, Wind and Ship Interaction}

Ship motion is predominately influenced by sea wave interactions, however, sea wave actions themselves are less deterministic. Ocean waves are the result of an accumulation of various types of waves including capillary waves generated from atmospheric pressure, wind waves, and planetary waves [19]. The bi-product of the accumulation of waves is the stochastic nature of sea motion, which is often described using an energy density spectrum. Wave spectrum intensifies as low frequency waves accumulate over time from the presence of wind, where eventually waves exhibit nonlinear energy transfer and are believed to travel up to or faster than the wind speed, known as a fully developed sea [20]. The spectral density $S(\omega)$ of a sea wave may be found using a Fast Fourier Transform (FFT). At a high level, the variance $\sigma_{\zeta}^{2}$ of wave surface elevation is found using a spectral moment analysis. A common term in sea keeping, significant wave height $H_{1 / 3}$, describes the average amplitude of the upper $30 \%$ of observed sea waves. Significant wave height may be solved for by using the standard deviation of wave elevation as

$$
H_{1 / 3}=4 \sqrt{\sigma_{\zeta}^{2}}
$$

The observed significant wave height may be used to find sea wave energy spectra, as 
well as estimate ocean winds. Assuming a fully developed sea, significant wave height may be correlated to wind speed at $19.5 \mathrm{ft} U_{19.5}$ and gravity $g$ using the PiersonMoskowitz wave model [20] as

$$
H_{1 / 3}=0.21 \frac{U_{19.5}^{2}}{g} .
$$

Further, Pierson-Moskowitz wave spectral density model for fully developed seas allows wave energy spectra to be modeled from angular wave frequency $\omega$, wind speed at $19.5 \mathrm{ft}$ and gravity g, such that the Pierson-Moskowitz wave model is

$$
S(\omega)=\alpha g^{2} \omega^{-5} \exp \left[-\beta\left(\frac{g}{\omega U_{19.5}}\right)^{-4}\right],
$$

where $\alpha=8.1(10)^{-3}$ and $\beta=0.74$. By deriving wave spectral density, sea waves may be modeled computationally allowing for the interaction between sea waves and ships to be analyzed. Ship motion may be modeled using a ship's Response Amplitude Operator (RAO), which is an estimated transfer function of a ship. RAOs are useful for ship design, ship analysis and the development of control strategies for traversing various sea ways [21]. The ship motion for this thesis was created using an RAO of a $30 \mathrm{~m}$ vessel traversing through various wave spectra. However, instead of using the Pierson-Moskowitz wave model, the Joint North Sea Wave Project (JONSWAP) model can be used. The JONSWAP model is based off the Pierson-Moskowitz model, however, JONSWAP assumes that the seas are never fully developed. The assumption presumes that the waves only develop over the length of the wind gusts, leading to more narrow spectral bandwidths with enhanced nonlinear effects [19]. The JONSWAP wave spectra equation is the same as equation 2.3 , but with an added peak enhancement factor $\gamma^{r}$. The base of the peak enhancement factor is a constant, $\gamma=3.3$, and its exponent $r$, is a ratio given by 


$$
r=\exp \left[-\frac{\left(\omega-\omega_{p}\right)^{2}}{2 \sigma_{p} \omega_{p}^{2}}\right],
$$

where, the peak frequency $\omega_{p}$ is solved as

$$
\omega_{p}=0.0879 \frac{g}{U_{19.5}}
$$

and the width of the peak region $\sigma_{p}$ is found using

$$
\sigma_{p}=\left\{\begin{array}{ll}
0.07, & \omega \leq \omega_{p} \\
0.00, & \omega>\omega_{p}
\end{array} .\right.
$$

The JONSWAP and Pierson-Moskowitz models are both accepted by oceanographers, and will produce the same result when $\gamma^{r}=1$. For this thesis, 105 sets of ship motion data were created for testing the landing performance of a UAV with ShipMo3D [22] using the JONSWAP spectrum. ShipMo3D was validated by the US Defense Technical Institute Center and was found to have a correspondence range of $10-30 \%$ to measured ship motion when comparing Root Mean Square (RMS) values $[23]$.

Describing wave conditions by their energy spectra is used in literature, however wave spectra is less tangible of a concept in gauging ocean activity for non oceanographers and marine scientists. Traditional but still widely accepted methods, such as the Douglas Sea Scale and Beaufort wind scale are commonly used in maritime literature [24]. The Douglas Sea Scale [25], devised in 1917 by English Admiral H.P. Douglas, was an attempt to standardize ocean roughness. The Beaufort sea scale [26], developed by Francis Beaufort, relates significant wave height to ocean winds. Table 2.1 outlines the sea states ranging from calm to phenomenal sea conditions from the Douglas Sea Scale and shows the equivalent Beaufort values. The ship motion created using ShipMo3D for use in this thesis ranges from sea state 2 to sea state 6 with 
wind velocities ranging between $3.4-15.4 \mathrm{~m} / \mathrm{s}$. In summary, wave energy increases from persistent wind gusts ultimately leading to increased significant wave height, thus the more intense the wave spectra, the higher the sea state and the less likely SHOL requirements will be met. Researchers are continually investigating a plethora of solutions to improve helicopter-ship landing performance.

Table 2.1: Douglas sea state [25] \& Beaufort wind scale [26].

\begin{tabular}{|c|c|c|c|c|c|}
\hline $\begin{array}{c}\text { Douglas } \\
\text { Sea } \\
\text { State }\end{array}$ & $\begin{array}{c}\text { Beaufort } \\
\text { Wind }\end{array}$ & $\begin{array}{c}\text { Wind Speed } \\
{[\mathbf{m} / \mathbf{s}]}\end{array}$ & $\begin{array}{c}\text { Signigicant } \\
\text { Wave Height } \\
{[\mathbf{m}]}\end{array}$ & Sea Desription & Wind Description \\
\hline 0 & 0 & $0-0.2$ & 0 & Calm & Calm \\
\hline 1 & 1 & $0.3-1.5$ & $0-0.1$ & Rippled & Light air \\
\hline 2 & 2 & $1.6-3.3$ & $0.1-0.3$ & Smooth & Light breeze \\
\hline 3 & 3 & $3.4-5.4$ & $0.3-1$ & Slight & Gentle breeze \\
\hline $3-4$ & 4 & $5.5-7.9$ & $0.7-1.5$ & Slight-moderate & Modertate breeze \\
\hline 4 & 5 & $8.0-10.7$ & $1.5-2.5$ & Moderate & Fresh breeze \\
\hline 5 & 6 & $10.8-13.8$ & $2.5-4$ & Moderate- rough & Strong \\
\hline $5-6$ & 7 & $13.9-17.1$ & $3.4-5.5$ & Rough-very rough & Neare gale \\
\hline $6-7$ & 8 & $17.2-20.7$ & $5-7.5$ & Very rough - high & Gale \\
\hline 7 & 9 & $20.8-24.4$ & $6-9$ & High & Severe gale \\
\hline 8 & 10 & $24.5-28.4$ & $8-12.5$ & Very high & Storm \\
\hline 8 & 11 & $28.5-32.6$ & $11-16$ & Very high & Violent Storm \\
\hline 9 & 12 & $32.7+$ & $14+$ & Phenomenal & Huricane \\
\hline
\end{tabular}

\subsection{Unmanned Landing Technology}

To improve landing manned, unmanned and autonomous helicopters, UAV-naval systems implement a combination of GPS, visual systems, and mounted radar and radio frequency beacons to orient the vehicle in relation to the ship deck [6]. Arora et al. [6] found success in using LIDAR and camera based control to track and land 
a helicopter on a ship without secondary deck mounted infrastructure. Sanchez et al. [27] suggest using a downward facing camera with a state estimator to robustly track ship motion for autonomous landing. Oh et al. [28] propose using a tether between a helicopter and ship to assist with landing, however, unlike a RAST system, the tether angle would be used to find the relative orientation between the helicopter and ship to facilitate with ship deck tracking. Alternatively, Kong et al. [29] used a deck mounted, infrared stereo vision system to track UAVs for autonomous landing. Kong et al. comment that their system should be fused with UAV sensor packages for better performance. However, infrastructure free landing is desired, thus Oh et al.'s and Kong et al.'s methods do not directly fit within the scope of this thesis.

Researchers have examined methods that allow for autonomous flight control, and landings on both stationary and moving decks. Garratt and Anavatti [30] used a neural network controller to produce heave trajectories for an unmanned helicopter. Similarly, Moriarty et al. [31] suggest using a stereo camera to track a ship's landing zone and a neural network to predict opportune landing windows. Hervas et al. [32] developed a landing control algorithm for unmanned vehicles on moving platforms that controlled the landing based solely on the relative heave motion between UAV and ship deck. While their simulations showed the algorithm was effective, the algorithm did not discriminate between safe landing times nor provide a method for tracking the ship trajectory. Ngo and Sultan [33] presented a model predictive control (MPC) design for helicopter shipboard operations in the presence of ship air wakes and rough seas. While the MPC method proved to be feasible in simulations, the researchers did not address the issue of the compatibility of their control method to other helicopter-ship combinations other than that which was simulated. Fourie [34] successfully used a Global Positioning System (GPS) for the autonomous landing of a remote control helicopter on a towed moving platform. Fourie's work was part of a larger initiative to use signal prediction to predict ship energy [35] to further the field 
in autonomous helicopter-ship landings. Fourie comments on the need to investigate and gauge the performance of landing systems and quantify when they should be used. Research in the field of autonomous tracking, control, and landing of UAV's is continuing to advance.

To improve the field, this research focuses on the development of a methodology for an infrastructure free, non-vehicle specific system, that facilitates UAV's in determining safe landing times while also compensating for the relative motion between the UAV and ship. Further, the methods used in this thesis, which are developed using position based control can be adapted for image based control.

\subsection{Ship Motion Prediction}

Forecasting ship motion on-line accurately can serve to improve daily maritime activities such as active ship stability, cargo transfer, fire accuracy of weapon systems, and the interfacing of aerial and ocean vehicles [36]. Despite the nonlinearity of ship motion, linear methods of forecasting such as Auto Regressive (AR) [37] and Auto Regressive Moving Average (ARMA) [38] have been used to predict ship motion, yet the precision from such estimators are questionable [36]. Zhao et al. [39] investigated using a Minor Component Analysis (MCA) prediction algorithm to predict ship motion that outperformed Neural Networks (NN) and an Auto Regressive (AR) model. For a 5 second prediction the NN and AR required 3.4 and 1.6 seconds of computational time on a $1.6 \mathrm{GHz}$ PC, compared to the 0.1 seconds of the MCA. The extended computational time for $\mathrm{NN}$ and $\mathrm{AR}$ draws into question the feasibility of implementing such systems for online estimation. Although outperforming the NN and the AR model the MCA required offline training using data sets. Requiring offline training may limit the performance for predicting ship motion produced by irregular waves.

Chung et al. [40] used a Kalman filter to estimate ship motion on-line through 
using estimated wave-excitation data as an input. The researchers estimated the wave-excitation information based off measured ship motion and used the ship's RAO to extrapolate future ship motion information. However, relying on knowledge of the ship's transfer function limits the compatibility of the system to be used for other vessels or on a UAV. Signal prediction independent of vessel type is currently used for active heave compensation on ships utilizing towing winches, allowing the system to actively pay-in or reel-out line to the towed object to reduce transmitted motion of submerged equipment [7]. The SPA utilizes an FFT and a linear Kalman filter to predict ship motion. Figure 2.3 is a schematic diagram of the SPA which was advanced by McPhee [8], where ship motion, such as roll, is observed over an evaluation window $T_{F F T}$ to initialize an FFT. The FFT and a peak detection algorithm are used to find the dominate modes $N$ and corresponding amplitude $A_{F F T}$, frequency $f_{F F T}$ and phase $\Phi_{F F T}$ of the signal. An observer is initialized to adapt $N$ mode parameters on-line, where the adapted modes are used to the forecast the signal over a prediction horizon $T_{\text {Pred }}$. The predicted signal $S_{\text {Pred }}$ is evaluated against a threshold value, if $S_{\text {Pred }}$ is below the threshold value over the prediction horizon, the SPA will output a Go signal. It is hypothesized that a similar motion prediction algorithm used by Woodacre et al. [7] and advanced by McPhee [8] could prove beneficial to UAV-ship landings by allowing UAVs to land within specified motion thresholds and improve landing efficiency by decreasing the number of landing attempts. Using the SPA to facilitate the landing of a UAV on a ship will be tested in simulation on a Quad Rotor (QR) helicopter. 


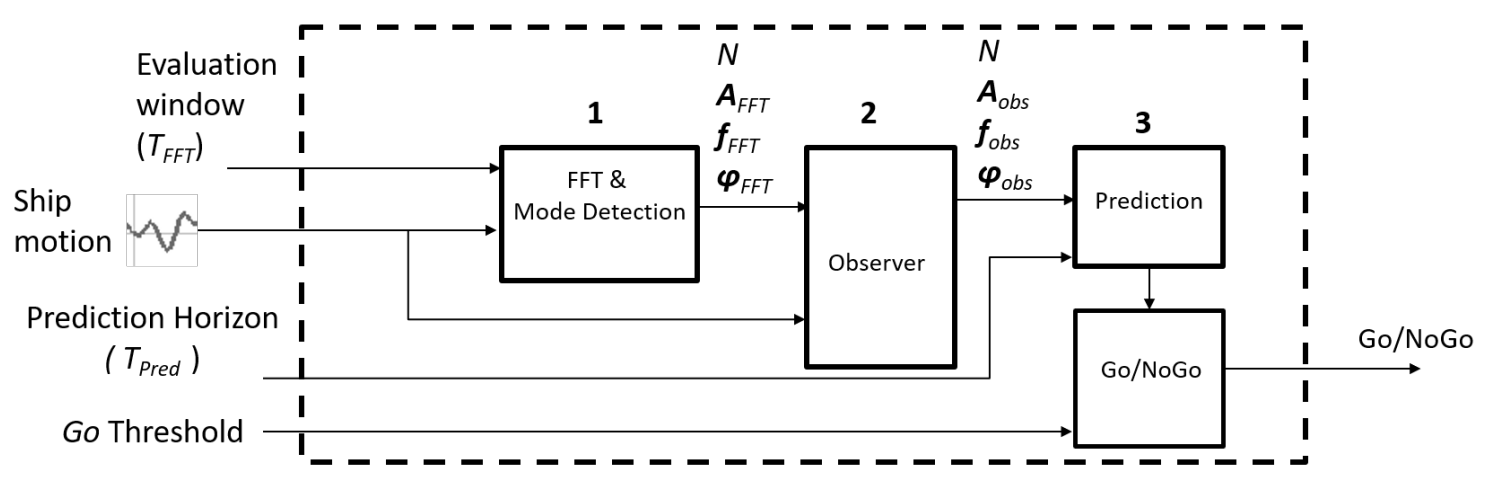

Figure 2.3: Information structure of the Signal Prediction Algorithm [9].

\subsection{Quad Rotor}

A QR was selected for this research due the relatively simple actuation and control in comparison to a standard helicopter. Further, the proof-of-concept is independent of the vehicle type, validating the use of a QR. QRs may very well find their place within maritime operations as portable QRs are gaining popularity in both military and civilian use.

Unlike standard helicopters which traditionally use dual counter-rotating overhead propellers or a single overhead propeller paired with a smaller tail propeller, quad rotors achieve yaw stability through four rotors that counter rotate to the propeller adjacent to them. Figure 2.4 illustrates the two most common structural configurations for quad rotors: + and $\times$. To yaw the aircraft clockwise, the speed of propellers 1 and 3 would be increased, while the speed of propellers 2 and 4 would be decreased, thus changing the net torque about the Centre of Gravity (CG) while still maintaining constant thrust. Assuming that the lateral axis is in line with propellers 2 and 4, to translate on the lateral axis, the thrust would be increased on either propeller 2 or 4, while decreased on the opposite propeller which ensures constant net thrust on the craft, while also rolling the vehicle. The same process would be used for longitudinal travel by pitching the UAV through manipulation of propellers 1 and 3 . 

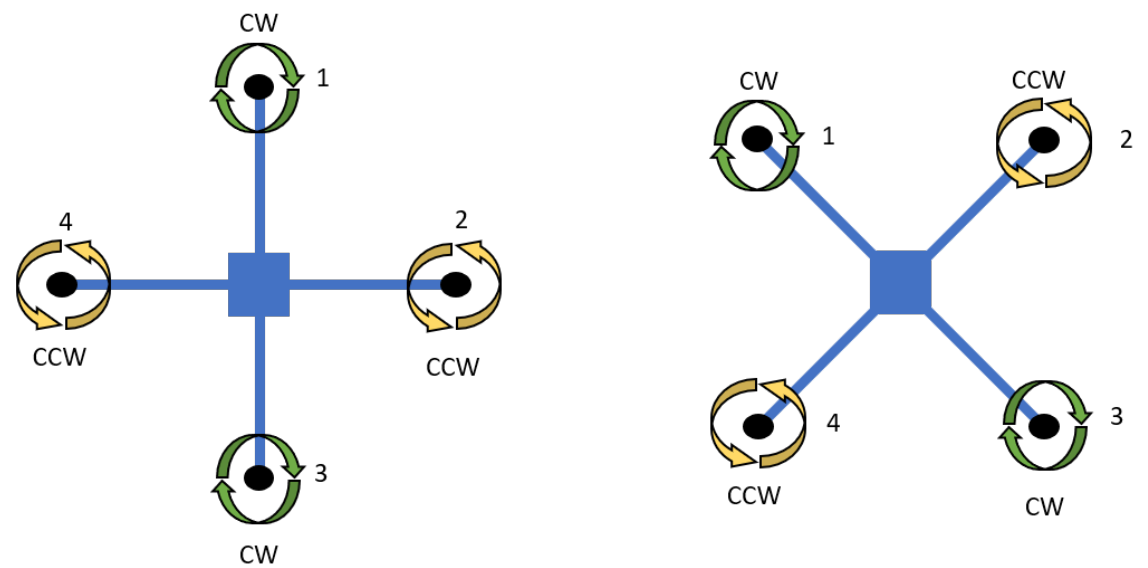

Figure 2.4: Typical quad rotor configurations. + (left),$\times$ (right)

\subsubsection{Quad Rotor Control}

Various control strategies have been developed and used to control quad rotors. Li et al. [41] were able to develop Proportional-Integral-Derivative (PID) controllers for a quad rotor in a Matlab Simulink model based off transfer functions derived from the QR dynamics through commissioning. A four channel (altitude, yaw, pitch, roll) PID control proved effective in reaching stability in response to a step input in simulation; however, the control gains did not translate to the physical system, which may be attributed to an inaccurate plant model. The use of Simulink to capture model nonlinearity and a QR with an accurate Computer-Aided Design (CAD) model may have improved their results.

Gautam et al. [42] developed a self-tuning PID controller using fuzzy logic. Their controller showed stronger control and disturbance rejection than a standard PID controller, while also exhibiting the ability to adapt controller gains to the flying environment. Despite their smart-selection algorithm, over 200 fuzzy parameters have to be tuned per time-step, thus requiring significant computational power versus a standard PID controller which has static gains. Gautam et al. do not comment on the real-time applicability of using a self-tuning fuzzy logic PID controller on a QR. Strong disturbance rejection and self-tuning controls would prove beneficial 
for an air-vehicle subjected to varying gust and atmospheric turbulence loading. A comparison between a PID controller and a Linear Quadratic Regulator (LQR) for a quad rotor was performed by Bouabdallah et al. [43]. The LQR performed similarly to the standard PID controller, though Bouabdallah et al. suggest the LQR will outperform the PID in wind gust environments and state that performance of their LQR may have been less than optimal due to model imperfections. However, the reliance on an accurate linearized plant model along with the more advanced control theory complicates the implementation of an LQR. Further, Salih et al. [44] found PID controllers to perform robustly in simulation and advocate the use of PID controllers for quad rotor control.

Companies such as PIX Hawk [45] and Ardupilot [46] sell flight controllers for QRs which implement PID control. Other researchers have also found success in QR control using standard PID control. Wang et al. [47] suggested implementing a cascade PID control scheme for the yaw, pitch and roll of a quad rotor to increase robustness and disturbance rejection. For attitude positioning, typical PID controllers compensate for angular position error, however, a cascade controller utilizes an inner angular velocity loop that operates at a higher frequency than the primary controller and exhibits faster disturbance rejection and set point convergence. The use of a cascade control structure is a favorable option due to less complex implementation and added performance. In this thesis, control strategies based off the theory discussed by Wang et al. [47] is used for QR control with satisfactory results. To complete the objectives of this thesis a simulator model must be developed.

\subsection{Modelling Methods}

Simulation modelling provides a powerful platform for developing, testing, and conducting research in all fields of engineering. Using simulators, engineers may rapidly 
design and test prototypes in a cost-effective and efficient manner, allowing for faster turn around time from conception to production. Further, simulations enable comprehensive analysis of structural systems and fluid dynamics which may be difficult to obtain, require extensive physical testing, or are dangerous to conduct. For instance, physical testing of the operating bounds of a helicopter may endanger the pilot and others. However, accurately modelling a helicopter and testing it in a synthetic environment is more cost effective and safer. After investigative modelling, physical implementation of the modelled system may be streamlined with fewer unforeseen repercussions.

Different methods exist for simulation modelling. Finite Element Modelling (FEM) is a common tool for structural and fluid analysis and is used to quantify physical phenomena such as stress, strain, loading, as well as heat and flow transfer. There are various methods for implementing FEM, however, the underling principle is the use of a mesh of linked finite elements, connected at nodes, to solve Partial Differential Equations (PDEs). The elements of the mesh, each with a set of governing equations, form a stiffness matrix. The solution of the stiffness matrix results in an approximate solution of the PDE. Although FEM methods may be used for fluid analysis, a more common method is Computational Fluid Dynamics (CFD). CFD uses finite volume method modelling derived from the Navier-Stokes equations to discretize and solve for PDEs [48]. CFD analysis more accurately captures the nonlinear behaviour of fluids by solving for the Navier-Stokes equations in a control volume, where if FEM was used the conservation of mass and momentum energy would need to be manually controlled [49]. FEM and CFD are both useful tools for helicopter and ship modelling and analysis. Hughes et al. [50] used FEM to predict the crashworthy response of helicopters onto water to improve upon what is currently offered - Similar FEM analysis on the landing gear of the QR would provide useful metrics on landing performance. Bauchau et al. [51] were successful in modelling helicopter 
dynamics using nonlinear finite element methods. Using a fully articulated dynamic FEM model, Bauchau et al. were able to analyze mechanical interaction parameters, such as stress, between helicopter components. Dynamic FEM analysis proves useful for testing and validation of helicopter design, however is inapplicable to the scope of this thesis. Further, the extreme computational cost of having a model with thousands of Degrees of Freedom (DoF) establishes a time constraint on model evolution and running batch simulations.

To increase the fidelity of a ship-helicopter model, CFD analysis can be used to find the interaction between oceanic winds and ship superstructures. Bogstad et al. were successful in mapping wind flow and induced vortices over a navy vessel for a flight simulator using CFD [52]. The authors comment that although CFD is not applicable to real-time simulations, the results from a CFD analysis may be used in a lookup table similar to empirical methods currently used. Figure 2.5 is an example of Bogstad et al.'s CFD model with a wind angle of $135^{\circ}$ relative to the bow of the ship. A similar CFD wind flow analysis would increase the fidelity of the simulator created for this thesis, however, requires a depth of knowledge in CFD implementation and significantly increase computational costs. Steliji et al. [53] described a framework to analyze helicopter rotor behaviour in hover and forward flight. CFD analysis of the propellers used on the quad rotor would require an accurate CAD model of the rotors, but could provide more accurate thrust modelling in the presence of wind gusts. It was assumed that the propeller data used for this thesis, which is based on empirically derived data, is sufficient for capturing rotor performance for the needs of basic flight. CFD and FEM may also be used together for joined structural and fluid analysis. Tomiki et al. were able to couple CFD and FEM analysis to estimate slamming impact pressure and resulting hydro-elastic response of a ship traversing in rough seas to enhance the structural safety of container ships [54]. Although a FEM-CFD model could enhance the validation of the ship motion used and provide 


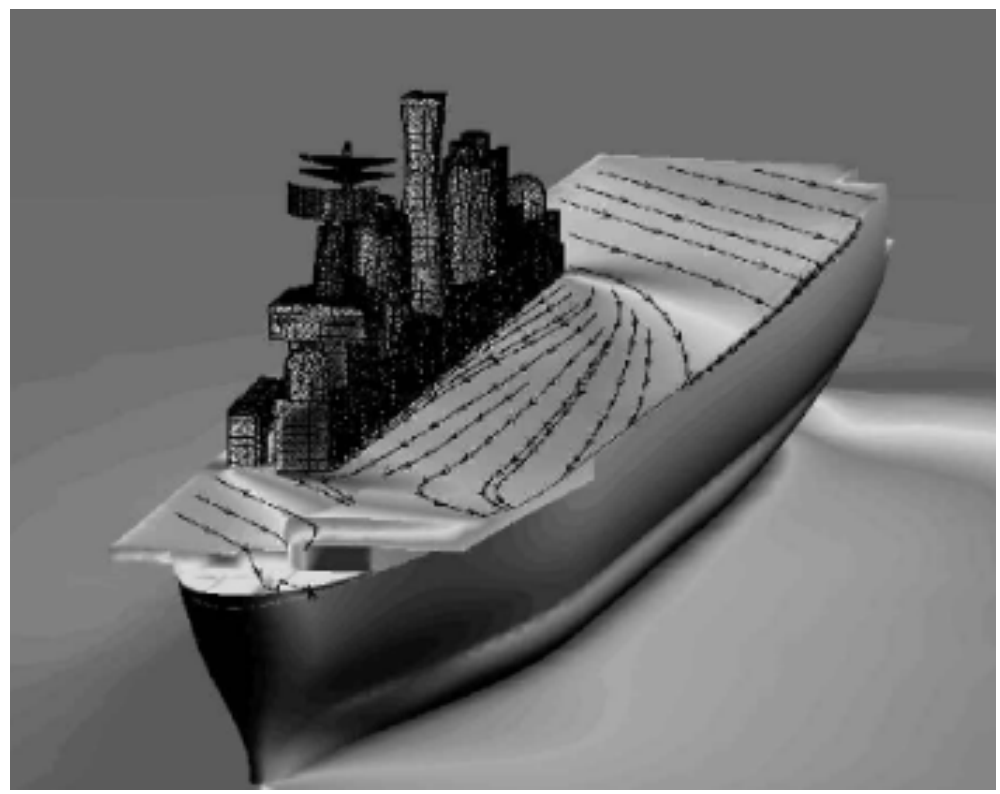

Figure 2.5: Simulated wind flow over a ship deck using CFD [52]

useful data on the forces imparted on the helicopter after landing, the current RAO used to model ship motion is sufficient for a simulator focused primarily on landing.

Another widely used method for simulating models is through state space representation. State space models capture dynamic system behaviour through the use of first order differential equations, although higher ordered systems exist, and are capable of accommodating multiple inputs and outputs [55]. State space models are efficient to compute and can be estimated for systems with unknown dynamics using system identification [56]. However, state space representation of models are predominately linear and require further linearization techniques to accurately model nonlinear systems. Ship RAO transfer functions are an example of a linear time-invariant state space model. State space representation is frequently found in literature for quad rotor dynamic modelling $[41,42,44]$. State space models are useful for designing control efforts for systems and evaluating system response, yet may be limited by linear approximations. State space models are useful sub-components of larger simulators where an approximation of system response is sufficient, or the exact 
operating bounds are known and linearized about, though, a simulator may not be comprised solely of state space models. State space models may be implemented in model based design environments such as Matlab's Simulink.

Simulink and other model based design platforms offer a modular work-space environment capable of integrating multiple simulation tools such as state space models, with other sub components: electronics, filters, software and hardware. Furthermore, Simulink automatically generates system equations based on specified system dynamics and model joint articulation, thus increasing model turnaround time while also capturing nonlinear system behavior. A powerful property of Simulink is the ability to generate $\mathrm{C}$ code from model structures. For example, flight software and control efforts designed in the work-space can be converted to $\mathrm{C}$ code and exported to physical hardware for validation and testing or used in other programs. Ribeiro et al. [57] were able to use an autopilot developed in Simulink to fly aircraft in X-plane by linking the two programs. The power to link Simulink with other programs for real-time applications is advantageous to other simulation methods such CFD and FEM which are computationally expensive and difficult to cross-integrate with other platforms. Integrated Simulink tool boxes, such as the Control System Designer toolbox, enhance the design of models. The Control System Designer toolbox expedites the tuning of controllers by providing comprehensive analysis of plant models and controllers. Minh et al. [58] were successful in developing the control efforts for a state space model of a QR using Simulink.

Furthermore, Simulink offers premade modules of code which may be used with existing systems to accelerate the creation of models. However, the code for premade modules may be inaccessible which may bottleneck model analysis. Another powerful property of Simulink is the model visualizer which automatically generates a 3D render of the model. The ability to visualize models allows for a more in depth analysis and faster trouble shooting. Simulink was chosen as the platform for this 
simulator due to its ability to develop and tune controllers, actuate CAD models, and integrate flight software and hardware in a single environment. Overall, simulations are incredibly powerful tools, yet their results are limited by the accuracy of the theory used and the fidelity of the model. Simulations are a great starting point for engineering work, however, results obtained in simulations will always need to be validated through physical testing.

\subsection{Summary}

In this chapter, the key challenges for landing aircraft on maritime vessels was described. An introduction to ocean wave modelling and ship motion was provided. In this thesis the JONSWAP spectrum is used for modelling waves and ShipMo3D is used to simulate ship motion. Relevant technology used for improving the seaworthiness of ocean-going helicopters was discussed including methods for predicting ship motion. In this work, a signal prediction algorithm used for marine applications is used to facilitate the landing of a UAV. The test-bed for this research is a quad rotor UAV which is simulated in an synthetic environment using Simulink model based design.

The following chapter describes the methodology used for creating the UAV model and simulator. 


\section{Chapter 3}

\section{Model \& Synthetic Environment}

In order to properly understand the impetus and methodology of this thesis, it is important to provide some exposition relating to the quad rotor UAV model employed in the simulation. In Section 3.1 a high level overview of the simulation is depicted in a flow diagram and the model components are expanded on. In Section 3.2 the motors are modelled and the propeller theory used is reviewed. Section 3.3 outlines UAV form and skin drag and the operating limits of the UAV. Atmospheric turbulence and wind gusts relating to sea states are introduced in section 3.4. Section 3.5 describes the PID control strategies implemented to control the UAV and track trajectories. In Section 3.6 two case studies evaluate the trajectory tracking performance of the UAV. The influence of sea states and ship motion on opportune landing periods is examined in Section 3.7. Finally, Section 3.8 derives the equations used to emulate the LIDAR used for detecting ship pose.

\subsection{Model Architecture}

The quad rotor UAV used in the simulation was sized to match similar vehicles in its class such as the DJI Phantom [59] and DJI Matrice 200 [60]. Figure 3.1 is a dimensioned front view drawing of the UAV that was developed for the simulation. 
The arms of the UAV extend $0.36 \mathrm{~m}$ (14 in) from the centre of the body, and carry LIDAR systems for ship pose detection. The DC motors are spaced $0.25 \mathrm{~m}$ (10 in) from the centre and are paired with 10 in propellers. The motors are modeled after Mega - RCn 600 motors [61] which have a velocity constant $K_{v}$ of $1370 \mathrm{RPM} / \mathrm{V}$ and are rated for $8.8 \mathrm{~V}$. The motor's theoretical rotational velocity is given by $\Omega_{m}=$ $\eta_{m} K_{v} V_{m}$, where $\eta_{m}=80 \%$ is the motor efficiency, and $V_{m}$ is the voltage supplied to the motor. The motors paired with 10 in propellers are able to generate approximately $14 \mathrm{~N}$ of thrust providing a 1.6 thrust-to-weight ratio for the vehicle. The modelling of the motors is further discussed in Section 3.2.1.

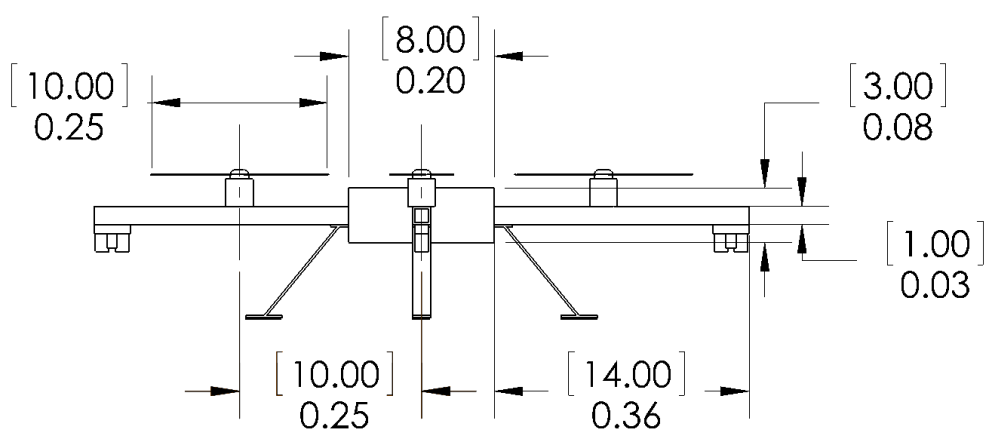

Figure 3.1: Front view dimensional drawing of UAV with units displayed in $\mathrm{m}$ and [in].

The simulation was developed in MATLAB's Simulink environment. Simulink allows for time domain simulations with subsystems operating at specified frequencies. As described in Section 2.6, Simulink enables modelling of the non-linear characteristics of the system and provides a platform for block and text based coding. Further, the modular construction of the simulator allows for simple substitution of other aircraft and vessel models into the synthetic environment.

Figure 3.2 is a high-level diagram of the information flow within the simulator model. The ship motion data, created using ShipMo3D, is imported into the simulation work space and used as a kinematic input to actuate the ship. The UAV, within the same model, launches a set distance behind the ship and actively tracks 
the position of the landing zone. During the simulation, external wind loading from atmospheric turbulence and wind gusts perturb the UAV. While above the landing zone, a sensor system measures the distance to the deck and relays that information to an algorithm for ship pose detection. The ship pose detection algorithm accounts for the orientation of the UAV and measurements of the sensor system. Ship pitch and roll angles are transferred to the SPA to predict when the ship motion is within the SHOLs for landing.

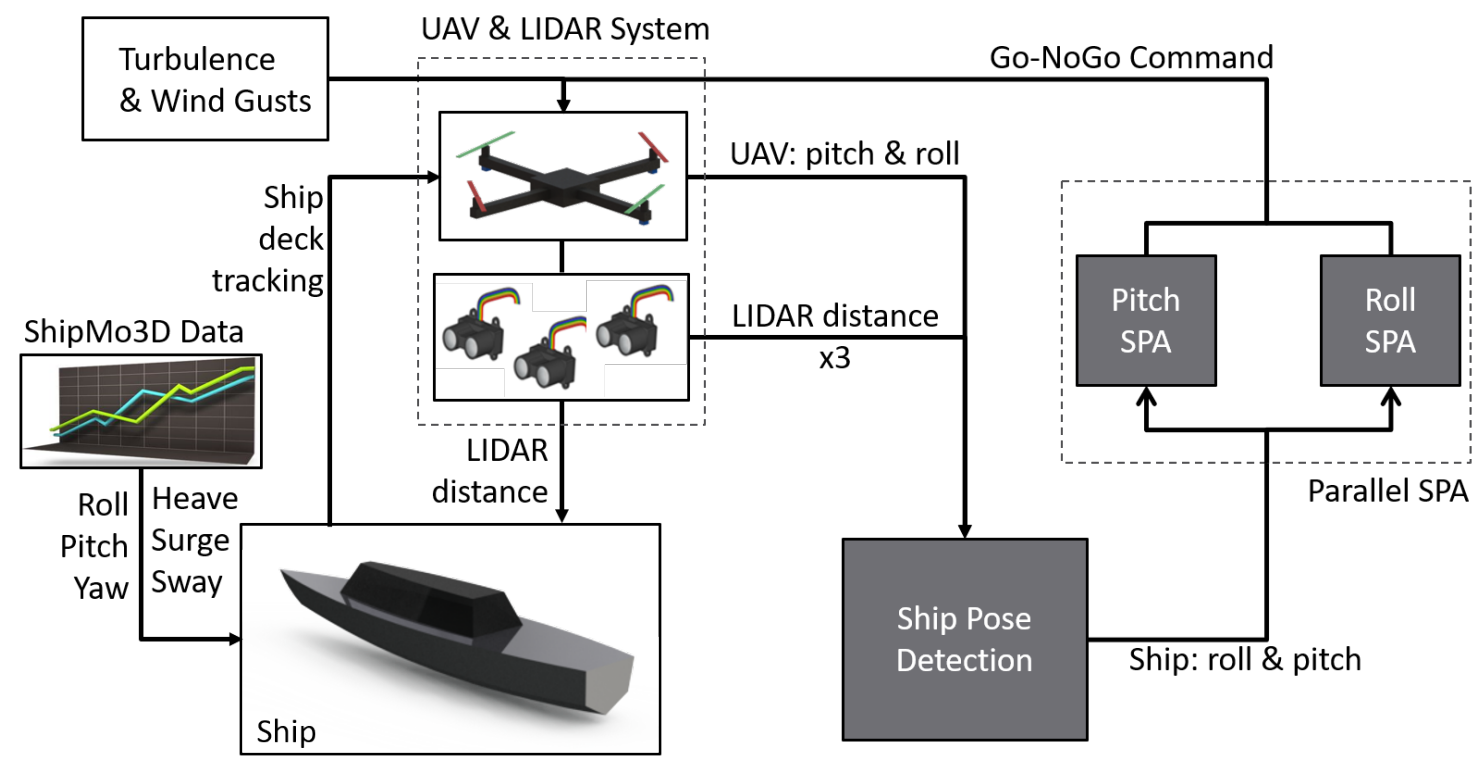

Figure 3.2: Model information flow within the simulator.

The main subsystems used for simulating the UAV are highlighted in Figure 3.3. The Trajectory Tracking controllers, Figure 3.3, are used for controlling the $\mathrm{x}$ and y positions of the UAV to enable ship tracking. Trajectory Tracking control signals are the reference angular positions for the UAV's roll and pitch controllers that minimize the distance error between the landing zone and the UAV. Within the UAV Control subsystems, the altitude, yaw, roll, and pitch controllers are highlighted. Each controller provides a unique control signal to the Motor Mixer, which combines the control signals and distributes the appropriate voltage to each motor. In the Motor \& Propeller subsystems, the control signals are converted 
into Pulse Width Modulated (PWM) signals and are used to drive the motors, the thrust and drag forces generated by the propellers are also considered in the same subsystem. Finally, the UAV Model \& Aerodynamics subsystem contains the CAD model of the UAV, information on the orientation and location of the UAV, as well as the sub-components for calculating the aerodynamic forces.

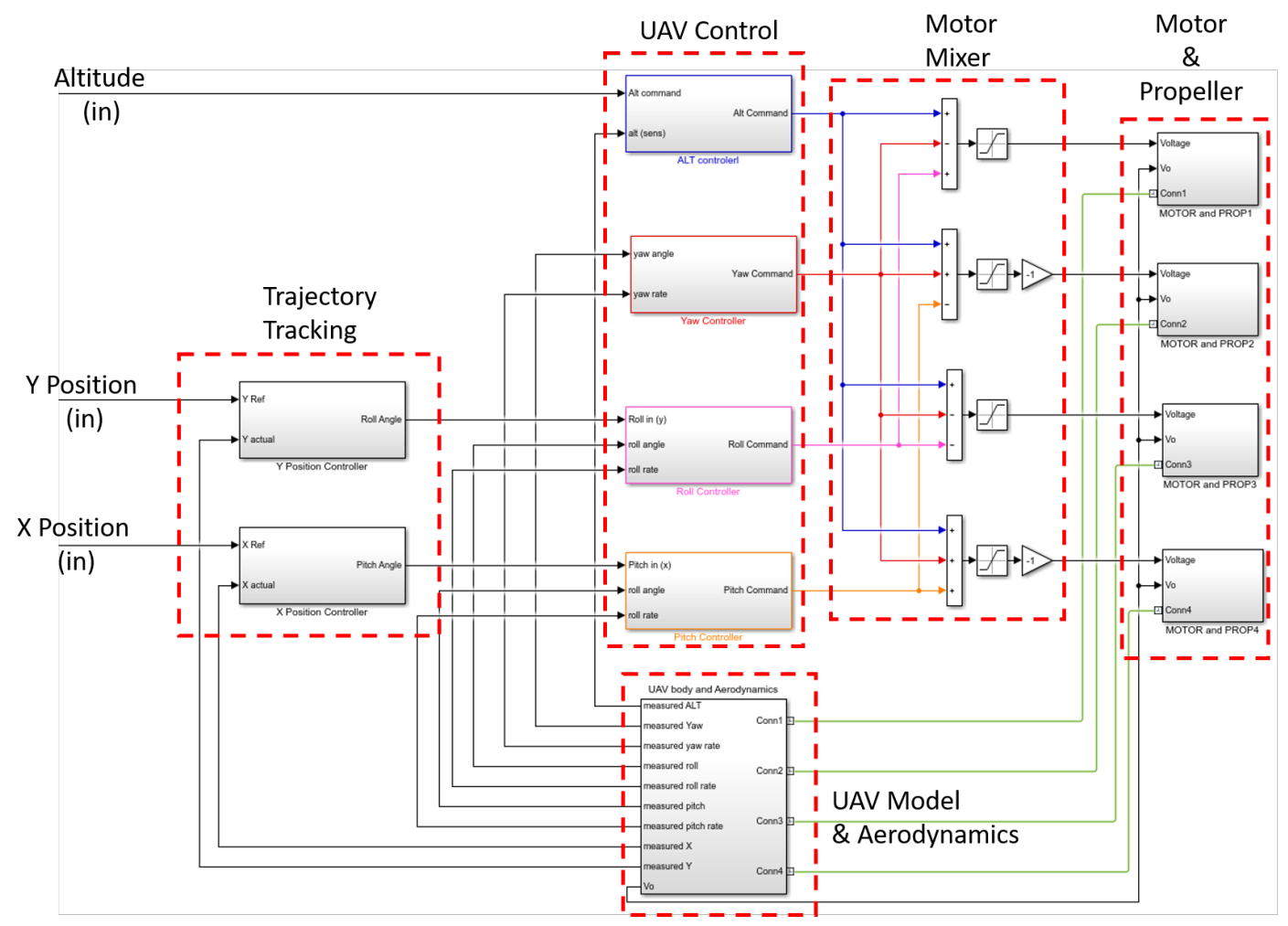

Figure 3.3: Subsystems of the quad rotor UAV model.

\subsection{Motor Modelling \& Propeller Theory}

Four motor-propeller combinations generate the lift and thrust to actuate the UAV. As specified in Section 3.1 the motors are $8.8 \mathrm{~V}$ RCn 600 motors and drive $10 \times 5$ in propellers. The motors are modeled as a DC motors with an H-bridge using Simscape within Simulink. The thrust produced by the propellers is calculated based on empirically derived data that correlates to motor speed and air inflow velocity. 


\subsubsection{Motor Modelling}

Figure 3.4 is the overall model of the motor, which includes the control signal, PWM generation, an H-bridge, and the motor model with propeller inertia and torque feedback. Table 3.1 highlights the parameters of the PWM signal, H-bridge, and motor. Initially the control signal which ranges from 0-100\%, and corresponds to the desired duty cycle, is converted into a $5 \mathrm{~V}$ PWM signal using the Controlled PWM Voltage block. The PWM signal enters the H-Bridge motor drive that has a total bridge resistance of $0.04 \Omega$. The $H$ bridge has a supply voltage of $8.8 \mathrm{~V}$ that is scaled based on the input voltage. The voltage polarity of the H-bridge may also be reversed to achieve counter rotation. The motor parameters are defined as inductance, no-load speed, rated speed, power, and rated voltage. The motor parameters match the values given by [61]. As the motor draws current and overcomes the inertia of the propeller the torque produced from propeller drag adds load to the motor. The motors draw a maximum current of 33.8 A. With the motors modeled, propeller theory can be examined.

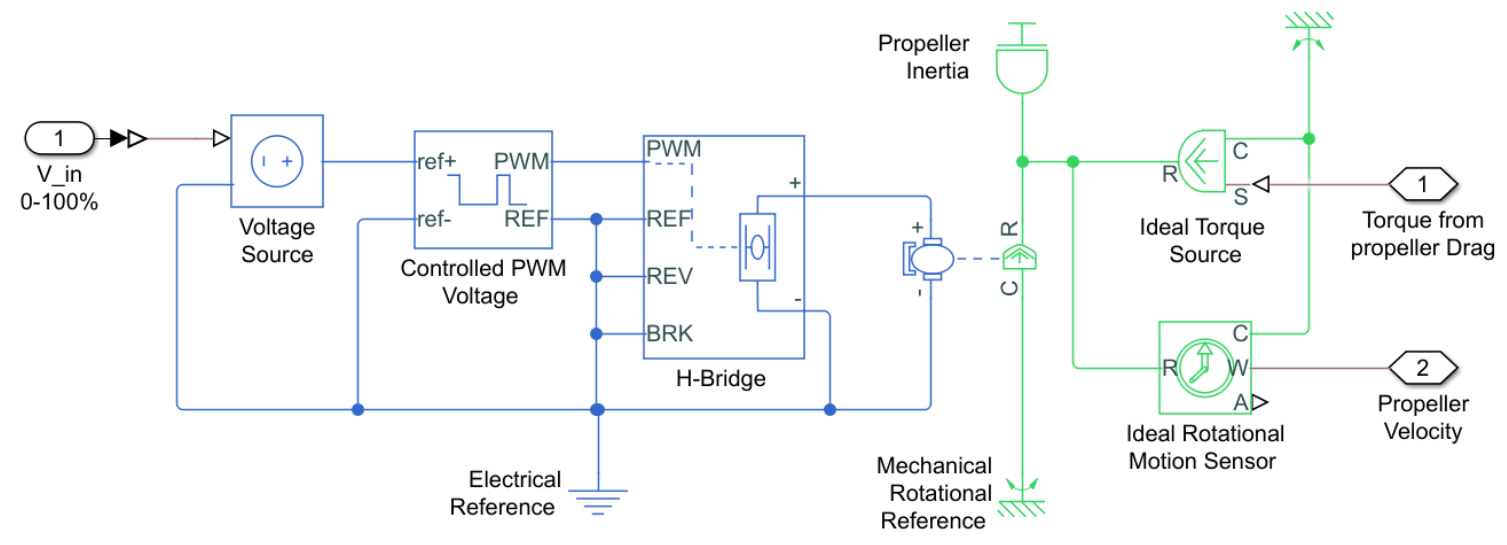

Figure 3.4: PWM controlled motor with torque feedback in Simscape. 
Table 3.1: Modeled PWM, H-bridge, and motor parameters.

\begin{tabular}{|c|c|c|c|c|c|}
\hline \multirow{2}{*}{ Controlled PWM } & Input Scaling & Output Voltage & & & \\
\hline & 0-100\% (control signal) & $0-5 \mathrm{~V}$ & & & \\
\hline \multirow{2}{*}{ H-Bridge } & Total Resistance & PWM Input & Output Voltage & & \\
\hline & $0.04 \Omega$ & $0-5 \mathrm{~V}$ & $0-8.8 \mathrm{~V}$ & & \\
\hline \multirow{2}{*}{ DC Motor } & Inductance & No-load Speed & Rated Speed & Power & Rated Voltage \\
\hline & $1 \mathrm{mH}$ & $12000 \mathrm{RPM}$ & $10,000 \mathrm{RPM}$ & $297 \mathrm{~W}$ & $8.8 \mathrm{~V}$ \\
\hline
\end{tabular}

\subsubsection{Propeller Theory}

For a propeller in a control volume, the only force on the control volume is a result of the change in momentum flux across its boundaries producing a thrust $T_{p}$, equated as

$$
T_{p}=\dot{m}\left(V_{e}-V_{o}\right)
$$

where, $\dot{m}$ is the mass flow rate of the air through the control volume, $V_{e}$ is the velocity of the air exiting the propeller and $V_{o}$ is the incoming airflow speed [62]. Further, noting that mass flow rate $\dot{m}$ is a function of air density $\rho$, propeller area $A_{p}$, and airflow differential $\left(V_{e}-V_{o}\right)$, Equation 3.1 may be expressed as

$$
T_{p}=\rho A_{p} V_{e}\left(V_{e}-V_{o}\right)=\rho A_{p} V_{e}^{2}-\rho A_{p} V_{e} V_{o}
$$

Propeller pitch $P_{p}$, the theoretical distance the propeller advances through the air per revolution, is a propeller parameter supplied by the manufacturer. Therefore, an approximate of the exit velocity of the air leaving the propeller $\hat{V}_{e}$ is found as a function of the angular velocity of the motor $\Omega_{m}$ and propeller pitch $P_{p}$, such that

$$
\hat{V}_{e}=\Omega_{m} P_{p}
$$

Equation 3.3, however, is not representative of the actual exiting air velocity as slip and propeller efficiency at different operating speeds are not considered. If the approximated exit velocity $\hat{V}_{e}$ is assumed to be equal to the actual exit velocity $V_{e}$, an 
equation for thrust based off motor speed and propeller pitch is

$$
T_{p}=\rho A_{p} \hat{V}_{e}\left(\hat{V}_{e}-V_{o}\right) .
$$

A comparison of the thrust calculated using Equation 3.4 to measured thrust confirmed that $\hat{V}_{e}=V_{e}$ is a poor assumption. Gabriel Staples (G.S.), a propeller enthusiast, developed a slip and efficiency propeller correction factor based on propeller pitch and diametre [63]. A propeller's efficiency is directly related to its Reynolds number which increases with the diametre of the propeller [64]. Further, the pitch of a propeller effects flow separation around the blade, where larger angles of attack induce greater flow separation, thus increasing induced drag and decreasing efficiency. From a data set of static propeller thrusts, Gabriel Staples used an iterative goal seeking approach to find a correction factor for Equation 3.4. The correction factor $\kappa_{p}$ was found to be

$$
\kappa_{p}=\left(\kappa_{1} \frac{d_{p}}{P_{p}}\right)^{\kappa_{2}},
$$

where $\kappa_{1}$ is the coefficient constant, $\kappa_{2}$ is the power constant which addresses the nonlinear relationship, and $d_{p}$ is the propeller diametre. Constants $\kappa_{1}$ and $\kappa_{2}$ were found to be a value of 0.303 and 1.5 [63]. The final thrust equation with G.S. correction is

$$
T_{p}=\rho A_{p} \hat{V}_{e}\left(\hat{V}_{e}-V_{o}\right) \kappa_{p} .
$$

To validate the derived propeller thrust equations propeller thrust data was experimentally collected for a generic $10 \times 5$ in propeller, 10 inches diametre with a pitch of 5 in, using a propeller test stand with a load cell and compared to theoretical thrust values obtained using Equations 3.4 and 3.6. Figure 3.5 plots the measured static thrust data for the generic $10 \times 5$ in propeller to the thrusts calculated using 
Equations 3.1 and 3.6. The solid black line shows the measured thrust, the dotted blue line shows the calculated thrust using Equation 3.1, and the dashed red line is the thrust calculated when including the G.S correction factor, Equation 3.6. The second subplot in Figure 3.1 compares the error between the calculated thrusts to the measured thrust, where the dotted blue line is the propeller thrust equation error and the dashed red line is the G.S. propeller thrust equation error. Comparing to the static thrust data, the average error was $5.5 \mathrm{~N}$ and $0.6 \mathrm{~N}$ for the propeller and G.S corrected propeller thrust equations respectively.
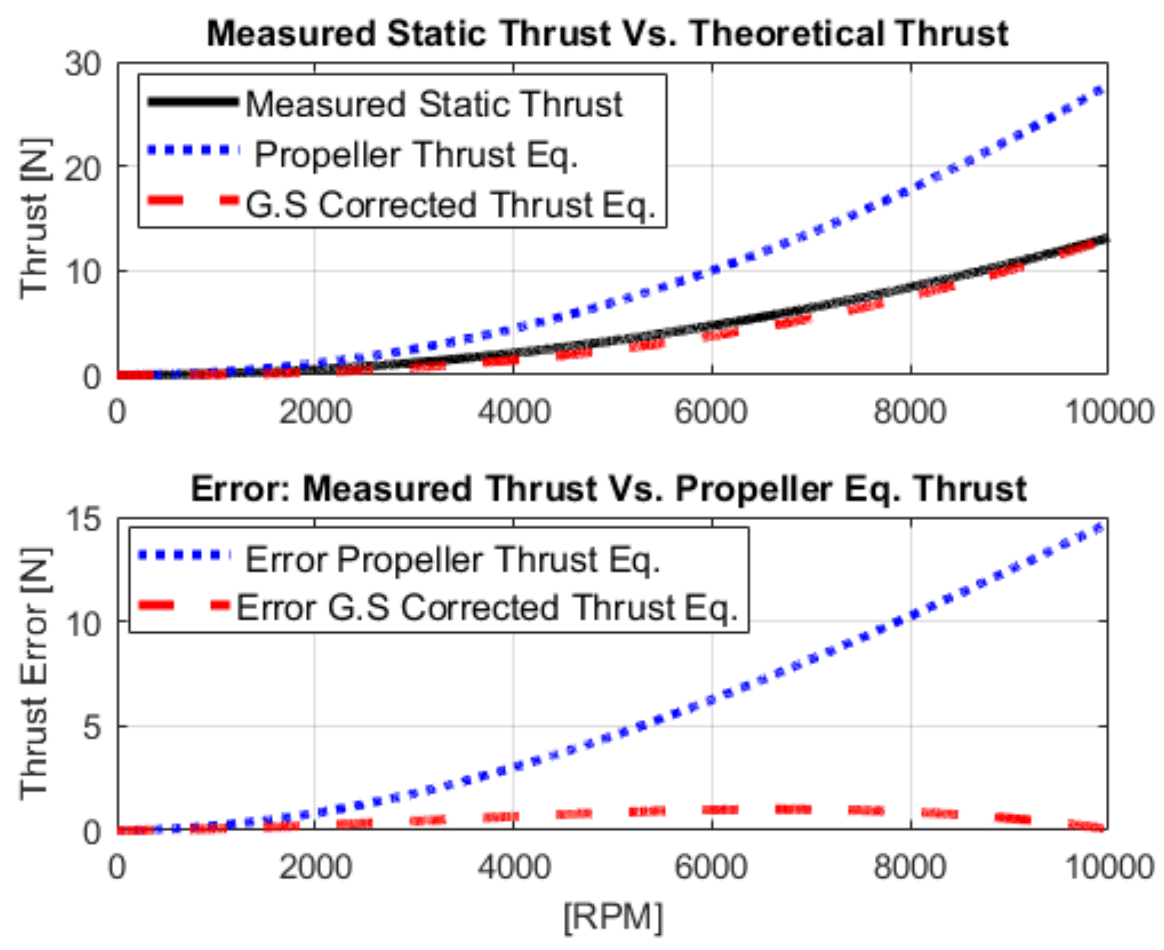

Figure 3.5: Static thrust and error comparison between measured data, theoretical propeller thrust equation and G.S corrected thrust equation.

Wind tunnel testing of a multitude of propellers was also performed by John Brandt and team from Arizona State University and University of Illinois UrbanaChampaign (UIUC) [65]. In their tests a variety of propellers were situated in a wind tunnel while attached to a sensing apparatus comprised of a load cell, a reaction torque transducer and a photo reflector. From their tests propeller power $P$ was 
found as a function air density $\rho$, wind tunnel velocity $V_{o}$ and motor speed $\Omega_{m}$ and were used to derive dimensionless propeller performance equations [65]. Performance parameters include the thrust coefficient $C_{T}$, power coefficient $C_{P}$, and efficiency $\eta_{p}$ which are a function of the advance ratio $J$

$$
J=\frac{V_{o}}{\Omega_{m} d_{p}},
$$

where $V_{o}$ is the airflow velocity of the wind tunnel, $\Omega_{m}$ is the angular velocity of the propeller, and $d_{p}$ is the propeller diametre. The thrust coefficient $C_{T}$, power coefficient $C_{P}$ and propeller efficiency $\eta_{p}$ are defined as

$$
\begin{gathered}
C_{T}=\frac{T_{p}}{\rho \Omega_{m}^{2} d_{p}^{4}}, \\
C_{P}=\frac{P}{\rho \Omega_{m}^{3} d_{p}^{5}}, \\
\eta_{p}=J \frac{C_{T}}{C_{P}} .
\end{gathered}
$$

The performance coefficients all relate to the propeller advance ratio and can be used to find the ideal operating envelope of a given propeller. For instance, Figure 3.6 highlights the performance parameters of a $10 \times 5$ in thin propeller. The coefficient of thrust, outlined by the black dashed line, decreases as the advance ratio increases. The coefficient of power, the blue dotted line, follows a similar trend. The peak efficiency of the propeller $\eta_{p}$, the solid orange line, shows that the peak efficiency for this propeller is obtained with an advance ratio between 0.3 and 0.6 . It is notable that when sizing propellers to pair with motors they should both have similar efficiency curves in their operating envelope [66]. 


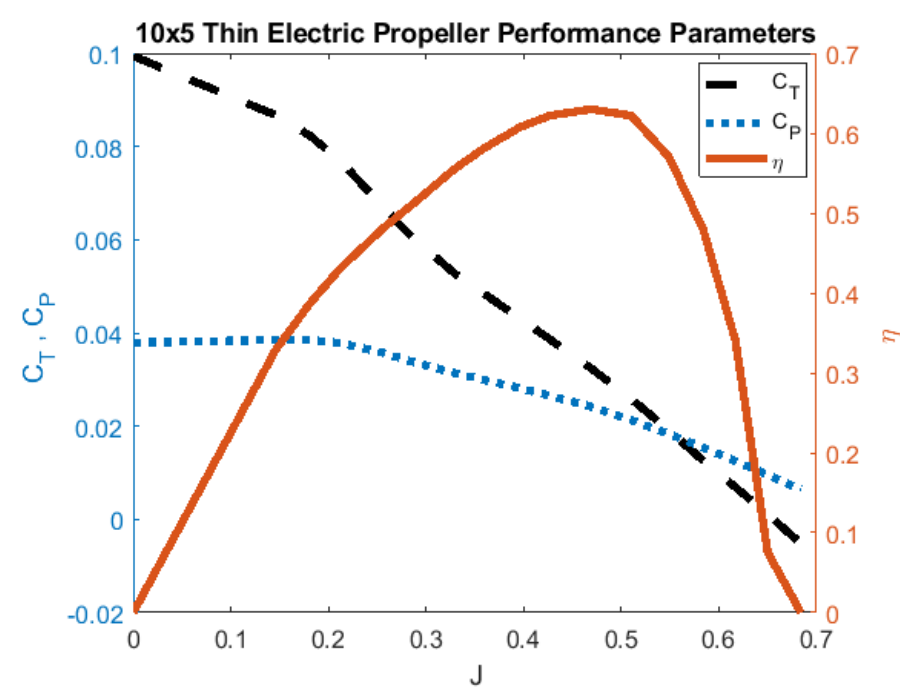

Figure 3.6: Propeller performance characteristics for a $10 \times 5$ in propeller.

Another parameter used in the model is propeller drag reaction torque $Q_{p}$. The torque is found by relating propeller power $P$ to motor velocity $\Omega_{m}$ by

$$
Q_{p}=\frac{P}{\Omega_{m}} .
$$

Substituting the above relation into Equation 3.9, the induced propeller torque $Q_{p}$ is found to be

$$
Q_{p}=C_{p} \rho \Omega_{m}^{2} d_{p}^{5} .
$$

The static thrust and torque values for a $10 \times 5$ in propeller are given in Figure 3.7, where the solid blue line is the thrust following the scale on the left y axis, and the dashed orange dashed line is the corresponding torque with the scale on the right $y$ axis. The static thrust and torque were found by solving for the advance ratio using Equation 3.7, finding the corresponding coefficients of thrust $C_{T}$ and power $C_{P}$, using a lookup table corresponding to Figure 3.6, and rearranging Equation 3.8 for thrust and Equation 3.12 for torque. 


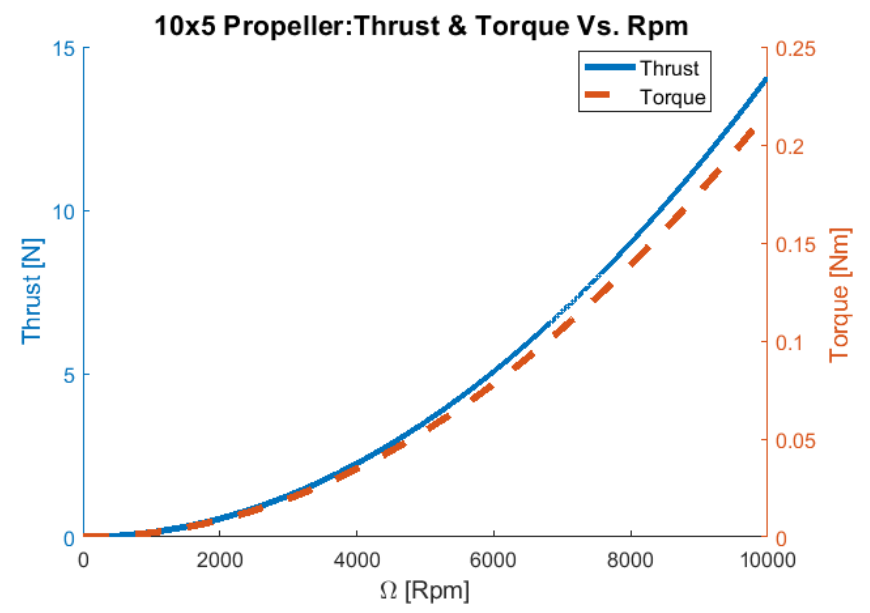

Figure 3.7: UIUC propeller $10 \times 5$ in propeller thrust and torque characteristics.

In Figure 3.8 the thrust values calculated using UIUC's thrust equation (Equation 3.8) as well as the G.S equation (Equation 3.6) are plotted against the measured static thrust. The solid black line is the measured thrust, the dashed red line is the G.S thrust, and the dotted blue line is the UIUC thrust. Although both G.S and UIUC's thrusts show good agreement with the measured data, UIUC had a better RMSE, $0.42 \mathrm{~N}$, when compared, to error of the G.S method, 0.75 N. Further, UIUC's data was empirically derived in wind conditions above what is expected in the simulation, thereby promising realistic thrust values in the model. As well, the ability to calculate drag induced torque is an added benefit for model fidelity. In this thesis, UIUC's propeller thrust and torque equations are implemented in the model.

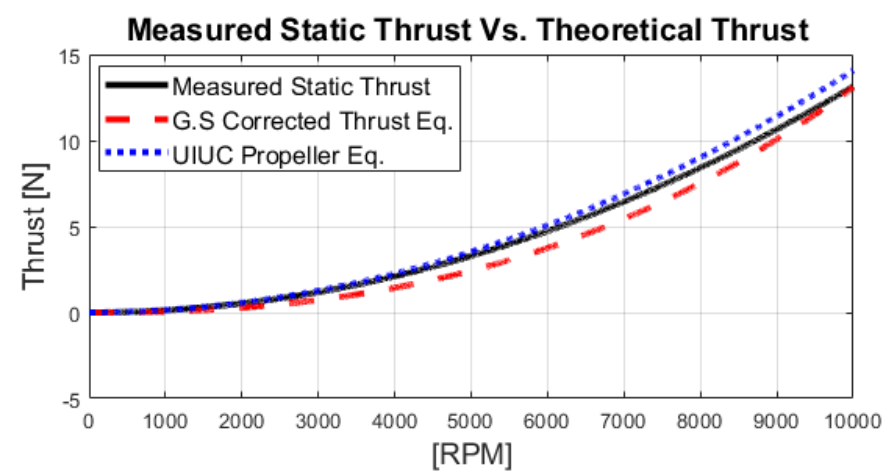

Figure 3.8: Static thrust comparison between measured data, theoretical calculations and empirically driven equations. 


\subsubsection{Ground Effect}

Ground effect was another aerodynamic phenomenon added to the model. Ground effect is a phenomenon experienced by both fixed wing and rotary wing aircraft which results in increased lift when the vehicle is in close proximity to the ground. It is well accepted that ground effect is the result of the propeller's slip-stream being deflected off the ground, ultimately reducing the velocity of the induced flow resulting in more lift [67]. Furthermore, the formation of rotor tip vorticies are obstructed by the ground which improves rotor performance. Typically, ground effect is encountered from the ground to a height of the propeller diametre [68]. Ultimately, propeller performance is increased by ground effect thus resulting in less power required to maintain thrust. Ground effect is often analyzed in terms of power requirements or thrust ratios. Cheeseman [68] empirically developed a thrust ratio $T R$ equation

$$
T R=\frac{1}{1-{\frac{d_{p}}{8 z_{U A V}}}^{2}},
$$

where $z_{U A V}$ is the distance to the ground. Cheeseman's thrust ratio is used in this thesis at propeller-height ratios of $z_{U A V} / d_{p} \leq 1$. In the simulation, the distance from each propeller to the ship deck is calculated and the thrust produced by that propeller is multiplied by $T R$. The next step in completing the simulator was formulating the UAV's aerodynamic properties.

\subsection{UAV Aerodynamics}

The drag force from the relative velocity between the UAV and the airfield is included in the simulator. The airfield is comprised of induced wind from UAV movement, wind gusts and atmospheric turbulence. The overall relative velocity between the UAV and the airfield is used to calculate the parasitic drag of the UAV. Parasitic 
drag is the combination of form and skin drag, where form drag is calculated based on the projected area of the UAV from the perspective of the relative wind velocity directional vector, and skin drag from the Reynolds number correlating to the relative wind velocity.

\subsubsection{UAV Form Drag}

To calculate the form drag, the relative wind velocity between the UAV and the airfield, referred to as True Air Speed (TAS) must be used. The TAS velocity $\vec{V}_{T A S}$ is calculated as

$$
\vec{V}_{T A S}=\vec{V}_{U A V}-\vec{V}_{W i n d}
$$

where $\vec{V}_{U A V}$ is the UAV's ground speed vector in the global frame, and $\vec{V}_{W i n d}$ is the atmospheric turbulence and wind gust velocity vector in the global frame. In order to find the projected area from the TAS perspective, a $3 \mathrm{D}$ point cloud $U A V_{x y z}$ representing the UAV follows two rotation sequences. The first rotation sequence orients the point cloud to match the UAV's position in the model, and the second to position the points from the perspective of the TAS directional vector $\hat{V}_{T A S}$. The unit TAS direction vector is found by

$$
\hat{V}_{T A S}=\frac{\vec{V}_{T A S}}{\left|\vec{V}_{T A S}\right|} .
$$

Rotation matrices corresponding to the UAV's Euler angles are used to rotate the point cloud from the UAV's body coordinates to global coordinates. Throughout this thesis rotations about the $\mathrm{x}, \mathrm{y}$ and $\mathrm{z}$ axes are denoted as $R_{x}, R_{y}$ and $R_{z}$ respectively, and follow the form 


$$
\begin{gathered}
R_{x}(\Phi)=\left[\begin{array}{ccc}
1 & 0 & 0 \\
0 & \cos (\Phi) & -\sin (\Phi) \\
0 & \sin (\Phi) & \cos (\Phi)
\end{array}\right], \\
R_{y}(\Theta)=\left[\begin{array}{ccc}
\cos (\Theta) & 0 & \sin (\Theta) \\
0 & 1 & 0 \\
-\sin (\Theta) & 0 & \cos (\Theta)
\end{array}\right], \\
R_{z}(\Psi)=\left[\begin{array}{ccc}
\cos (\Psi) & \sin (\Psi) & 0 \\
-\sin (\Psi) & \cos (\Psi) & 0 \\
0 & 0 & 1
\end{array}\right],
\end{gathered}
$$

where the rotation angles of matrices $R_{x}, R_{y}$ and $R_{z}$ are $\Phi, \Theta$ and $\Psi$ respectively, and follow the coordinate frame given in Figure 1.1. Using 3 rotation matrices the body frame point cloud $U A V_{x y z}$ is rotated to the global frame by

$$
{ }^{G}[U A V]_{x y z B}=R_{z}\left(\Psi_{U A V}\right) R_{y}\left(\Theta_{U A V}\right) R_{x}\left(\Phi_{U A V}\right)[U A V]_{x y z} .
$$

The subscript $B$ indicates the body frame and super script $G$ denotes the global frame, and the rotation is from its body frame to the global frame.

The UAV point cloud, now in global coordinates, is rotated once more to be orthogonal with vector $\hat{V}_{T A S}$. The rotation for the orthogonal projection can be accomplished with two rotation matrices corresponding to the angles that direction vector $\hat{V}_{T A S}$ forms between the principle axes. A z rotation accounts for the $\mathrm{x}$ and $\mathrm{y}$ components of $\hat{V}_{T A S}$, followed by a rotation about the x axis for the z component. The 
$\mathrm{z}$ axis rotation angle $\Psi_{\hat{V}_{T A S}}$ is found by taking the arctan of the $\mathrm{x}$ and $\mathrm{y}$ components of $\hat{V}_{T A S}$,

$$
\Psi_{\hat{V}_{T A S}}=\arctan \left(\frac{\hat{V}_{T A S x}}{\hat{V}_{T A S y}}\right) .
$$

The second rotation uses the angle formed between the $\mathrm{z}$ component of the TAS vector and its $\mathrm{x}$ and $\mathrm{y}$ components, solved for by

$$
\Phi_{\hat{V}_{T A S}}=\arccos \left(\hat{V}_{T A S z}\right) .
$$

The UAV point cloud is rotated to the perspective of the TAS directional vector through

$$
{ }^{T A S}[U A V]_{x y z G}=\left(R_{z}\left(\Psi_{\hat{V}_{T A S}}\right) R_{x}\left(\Phi_{\hat{V}_{T A S}}\right)\right)^{T}{ }^{G}\left[U A V_{x y z}\right]_{B}
$$

where, the rotation is from the global frame to the perspective of the TAS. There are three column vectors, $\vec{P}_{U A V_{x}}, \vec{P}_{U A V_{y}}, \vec{P}_{U A V_{z}}$ that make up the matrix ${ }^{T A S}[U A V]_{x y z G}$. The projected area is bounded by the border formed between the local maximums and minimums of $\vec{P}_{U A V x}$ and $\vec{P}_{U A V y}$. In the Simulink model, the $2 \mathrm{D}$ projected area $A_{2 D}$ was found using Matlab's boundary function to find the x-y border points followed by the polyarea function to calculate the bounded area. Figure 3.9a illustrates the projected area found using the TAS direction vector and the rotated UAV point cloud for a UAV during take off with no cross winds, here the projected area is only from the $\mathrm{z}$ axis prospective. Figure $3.9 \mathrm{~b}$ is the $2 \mathrm{D}$ projected area for a TAS in the $\mathrm{x}$ direction with a $\mathrm{UAV}$ pitched at $25^{\circ}$ resulting in a $2 \mathrm{D}$ projection of the front of the UAV. In Figure 3.9c, the UAV is pitched and rolled $25^{\circ}$ with equal $\mathrm{x}$ and $\mathrm{y}$ TAS components, which gives an isometric projection. The boundary, shown in the plots as a red line, may bound extra area between the fuselage and the arms increasing the total projected area. However, the added area is minimal with respect to the 


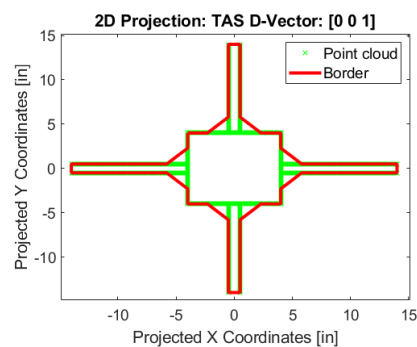

(a)

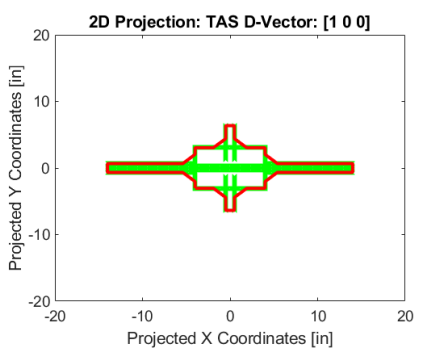

(b)

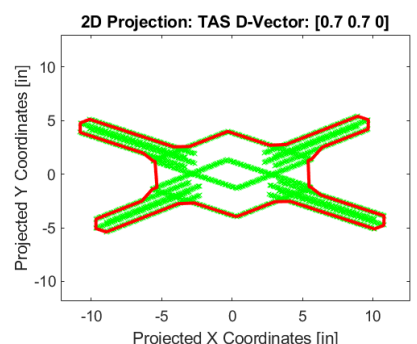

(c)

Figure 3.9: (a) 2D projection of UAV during accent with no cross winds. (b) 2D projection of pitched UAV travelling in head wind. (c) Rolled and pitched UAV traveling with equal $\mathrm{x}-\mathrm{y}$ velocity components.

total area and is assumed to have negligible contribution to the drag force. In the simulator, the point cloud rotation sequence is performed at every time step interval to ensure the projected area is representative of the UAV during flight.

Drag force $\vec{F}_{D \text { form }}$ is found using the 2 D project area of the UAV $A_{2 D}$, the magnitude of the TAS, and the TAS direction vector by

$$
\vec{F}_{D \text { form }}=\frac{1}{2} \rho\left|\vec{V}_{T A S}^{2}\right| C_{D b o d y} A_{2 D} \hat{V}_{T A S}
$$

where $\rho$ is air density and $C_{D b o d y}$ is the coefficient of drag. For the projected area, the coefficient of drag was taken as 0.8 , which is the coefficient of drag of a cube rotated $45^{\circ}$. The drag force $\vec{F}_{D \text { form }}$ does not account for the lift generated by the top of the UAV during flight. The top of the UAV was considered a flat plate, where when angled towards the wind, such as when in flight, will generate a negative lift pushing the UAV downward. Ortiz et al. [69] derived the relation between the Angle of Attack (AoA) $\alpha_{p}$ of a flat plate to its coefficient of lift. The coefficient of lift for a plate $C_{L_{p}}$ is defined as

$$
C_{L_{p}}=0.6 \sin (2 \alpha)
$$

The AoA of the UAV is taken as the angle between the UAV and the wind direction 


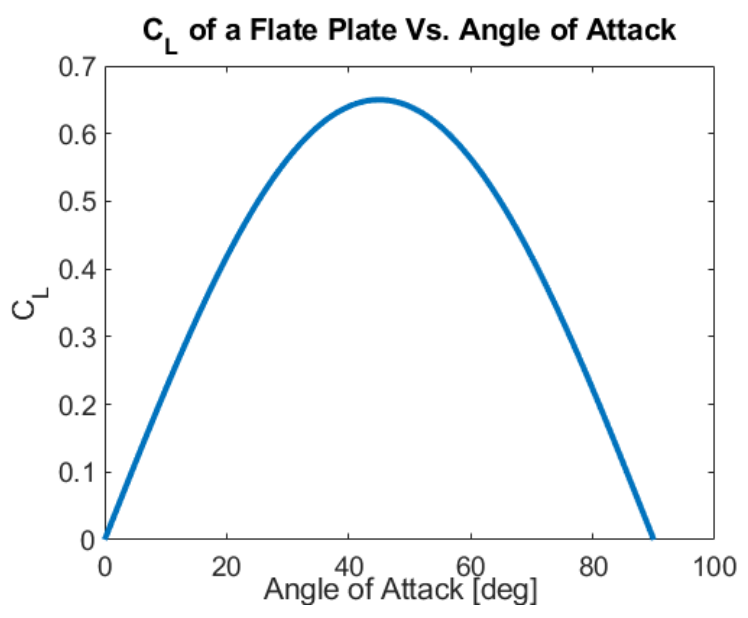

Figure 3.10: Coefficient of lift Vs. angle of attack for a flat plate.

vector. The lift generated by airflow over the top of the UAV $F_{L p}$ is expressed as

$$
F_{L p}=\frac{1}{2} \rho\left(\vec{V}_{T A S x}^{2}+\vec{V}_{T A S y}^{2}\right) C_{L_{p}} A_{p},
$$

where $A_{p}$ is the area of the top of the UAV's fuselage and arms. The influence of the AoA on the coefficient of lift is highlighted in Figure 3.10, the coefficient of lift follows a sinusoidal shape, with minimum values at $0^{\circ}$ and $90^{\circ}$, and reaches a maximum coefficient of lift at $45^{\circ}$ [70]. In a head wind, the lift generated is in the negative direction which exerts loading on the air frame, however in tail winds, the lift generated will decrease load on the motors. With form drag and lift analyzed, skin drag must also be examined.

\subsubsection{UAV Skin Drag}

Skin drag is also considered as a retardant force on the UAV, however due to the small surface area of the UAV, skin drag is almost negligible. To calculate the skin drag the Reynolds $R_{e}$ number across the fuselage is found using

$$
R_{e}=\frac{\left|\vec{V}_{T A S}\right| l_{\text {body }}}{v_{\text {air }}}
$$


where $l_{\text {body }}$ is the length of the fuselage, and $v_{a i r}$ is the kinematic viscosity of air. The coefficient of skin friction is then calculated using the Blasius friction law [71] which states

$$
C_{D \text { skin }}=\frac{1.328}{\sqrt{R_{e}}} .
$$

The skin friction drag force $\vec{F}_{D s k i n}$ is

$$
\vec{F}_{D s k i n}=\frac{1}{2} \rho \vec{V}_{T A S}^{2} A_{w e t} C_{D s k i n},
$$

where $A_{\text {wet }}$ is the wetted (total) surface area of the UAV. The net aerodynamic forces acting on the UAV is found through the summation of the form drag force, the lift generated by top of the UAV in the z direction and skin drag by

$$
\vec{F}_{U A V}=\vec{F}_{D f o r m}+\left[\begin{array}{c}
0 \\
0 \\
F_{L p}
\end{array}\right]+\vec{F}_{D s k i n} .
$$

With the propellers modeled in the last section and the drag forces established in this current section, the operating limits of the UAV can be found. Table 3.2 highlights the maximum operating bounds of the UAV, the corresponding propeller inflow velocities and drag forces. During take off the maximum rate of ascent is found to be $8.93 \mathrm{~m} / \mathrm{s}$, which is the maximum propeller inflow velocity before loss of thrust. For single axis traversal, at the UAV's maximum roll angle $\left(25^{\circ}\right)$, the y velocity was $16.8 \mathrm{~m} / \mathrm{s}$. The flow over the propeller did not reach the maximum inflow rate, indicating higher speeds may possibly be reached if the roll angle was increased. During forward flight, extra loading is applied on the UAV from overtop airflow, requiring added lift to remain at a constant altitude and lowering the 
maximum propeller inflow velocity before thrust is lost. While traversing in the $\mathrm{x}$ and y plane, the UAV was capable of reaching $20^{\circ}$ roll and pitch angles before the inflow velocity to the propellers exceeded the limit resulting in loss of altitude. The rotation sequences of the joints in the model influences the results of the maximum $\mathrm{x}-\mathrm{y}$ traversal velocities. In the model, the UAV's body is connected to the world frame by a series of revolute joints, a prismatic joint and a rectangular joint. For the revolute joints the rotation sequence follows roll then pitch then yaw, which enables a higher maximum y velocity than x velocity. Although an anomaly, the imbalance in maximum velocities does not hinder the UAV as it rarely travels at maximum speeds in both the $\mathrm{x}$ and $\mathrm{y}$ directions. The last row of Table 3.2 quantifies the maximum climb rate while traversing with $15^{\circ}$ roll \& pitch angles. The UAV is able to climb at $2 \mathrm{~m} / \mathrm{s}$, however, the downward $\mathrm{z}$ forces lower the maximum propeller inflow velocity before loss of thrust. From the values in Table 3.2 the UAV's TAS limits in wind gust conditions may be accessed using Equation 3.14. For instance, in a $10 \mathrm{~m} / \mathrm{s}$ head wind the UAV's maximum ground speed is $6.82 \mathrm{~m} / \mathrm{s}$. The following section investigates atmospheric turbulence and wind gusts pertaining to the synthetic environment in which the UAV will fly in.

Table 3.2: Operational limits of UAV.

\begin{tabular}{|l|c|c|c|c|c|c|c|c|c|}
\hline Flight Manoeuvre & $\begin{array}{c}\text { Roll } \\
\text { Angle } \\
{[\mathbf{d e g}]}\end{array}$ & $\begin{array}{c}\text { Pitch } \\
\text { Angle } \\
{[\mathbf{d e g}]}\end{array}$ & $\begin{array}{c}\text { TAS X } \\
{[\mathbf{m} / \mathbf{s}]}\end{array}$ & $\begin{array}{c}\text { TAS Y } \\
{[\mathbf{m} / \mathbf{s}]}\end{array}$ & $\begin{array}{c}\text { TAS Z } \\
{[\mathbf{m} / \mathbf{s}]}\end{array}$ & $\begin{array}{c}\text { Propeller } \\
\text { Inflow } \\
\text { Vel. }[\mathbf{m} / \mathbf{s}]\end{array}$ & $\begin{array}{c}\text { X drag } \\
{[\mathbf{N}]}\end{array}$ & $\begin{array}{c}\text { Y drag } \\
{[\mathbf{N}]}\end{array}$ & $\begin{array}{c}\text { Z drag } \\
{[\mathbf{N}]}\end{array}$ \\
\hline Max rate of ascent & 0 & 0 & 0 & 0 & 8.93 & 8.93 & 0.00 & 0.00 & 3.14 \\
\hline $\begin{array}{l}\text { Max single axis } \\
\text { traverse speed }\end{array}$ & 25 & 0 & 0 & 16.82 & 0 & 7.12 & 0.00 & 10.23 & 6.84 \\
\hline $\begin{array}{l}\text { Max x-y axes } \\
\text { traverse speeds at } \\
20^{\circ} \text { roll \& pitch }\end{array}$ & 20 & 20 & 11.16 & 11.93 & 0 & 8.00 & 8.09 & 8.65 & 7.18 \\
\hline $\begin{array}{l}\text { Max ascent \& x-y } \\
\text { traverse speeds at 15 } \\
\text { roll \& pitch }\end{array}$ & 15 & 15 & 10.79 & 11.16 & 2.09 & 7.54 & 6.04 & 6.25 & 7.58 \\
\hline
\end{tabular}




\subsection{Wind Modelling}

To increase the verisimilitude of the synthetic environment wind gusts and atmospheric turbulence matching the sea states were emulated. It is accepted that there is a strong correlation between wind speed and wave actions [72], and as such the wind in the model is correlated to the sea's significant wave height $H_{1 / 3}$ by rearranging Equation 2.2 from Section 2.2 as

$$
U_{19.5}=\sqrt{4.76 H_{1 / 3} g},
$$

where $U_{19.5}$ is the wind velocity at $19.5 \mathrm{ft}$ and $g$ is gravitational acceleration. The fetch, the length of the gust, is found as

$$
X_{w}=\frac{\widetilde{X_{w}}\left(U_{19.5} 1.026\right)^{2}}{g},
$$

where $\widetilde{X_{w}}$ is the fetch length of a fully developed wind $\left(\widetilde{X_{w}} \approx 2(10)^{4} \mathrm{~m}\right)$ [73]. Table 3.3 expresses the calculated expected wind velocities and fetch lengths using equations 3.30 and 3.31 with the significant wave heights used when creating the ship motion. The sea states range from 2-6, where sea state 2 has the lowest velocity and the smallest fetch and sea state 6 has the greatest wind velocity and fetch. Although residual waves persist after wind gusts subside, in this thesis it is assumed that the UAV will always encounter wind matching the sea state. The wind and fetch values from Table 3.3 are used for modelling wind gusts and atmospheric turbulence for the simulator.

\subsubsection{Wind Gusts}

Abiding by Military Specification MIL-F-8785C [74], wind gusts are modeled as a one minus cosine shape as portrayed in Figure 3.11, where the gust starts at a stand still 
and accelerates until it reaches a maximum velocity of $3.74 \mathrm{~m} / \mathrm{s}$. For an aircraft, the gust velocity $V_{\text {gust }}$ at a point in flight is taken as

$$
V_{\text {gust }}=\frac{1}{2} U_{19.5}\left(1-\cos \left(\frac{\pi x_{\text {gust }}}{x_{\text {gustMax }}}\right)\right)
$$

where $U_{19.5}$ is the wind velocity found in Table $3.3, x_{\text {gust }}$ is the distance the UAV has traveled into the wind field, and $x_{\text {gustMax }}$ is the distance the UAV has to travel to reach the maximum velocity. To quickly subject the UAV to the maximum expected wind velocities $x_{\text {gustMax }}$ is taken as $100 \mathrm{~m}$ in the model. The length of the fetch for all sea states is vastly greater than the maximum distance the UAV will travel in

Table 3.3: Expected wind velocities and fetch lengths for sea states 2-6.

\begin{tabular}{|l|l|l|l|}
\hline Sea State & $\begin{array}{l}\text { Significant Wave } \\
\text { Height } \mathbf{( m )}\end{array}$ & $\begin{array}{l}\text { Wind Velocity } \\
\mathbf{( m / s})\end{array}$ & $\begin{array}{l}\text { Fetch } \\
\mathbf{( k m})\end{array}$ \\
\hline 2 & 0.30 & 3.74 & 27.14 \\
\hline 3 & 0.88 & 6.40 & 60.62 \\
\hline 4 & 1.88 & 9.37 & 170.09 \\
\hline 5 & 3.75 & 13.24 & 339.27 \\
\hline 6 & 5.00 & 15.28 & 452.36 \\
\hline
\end{tabular}

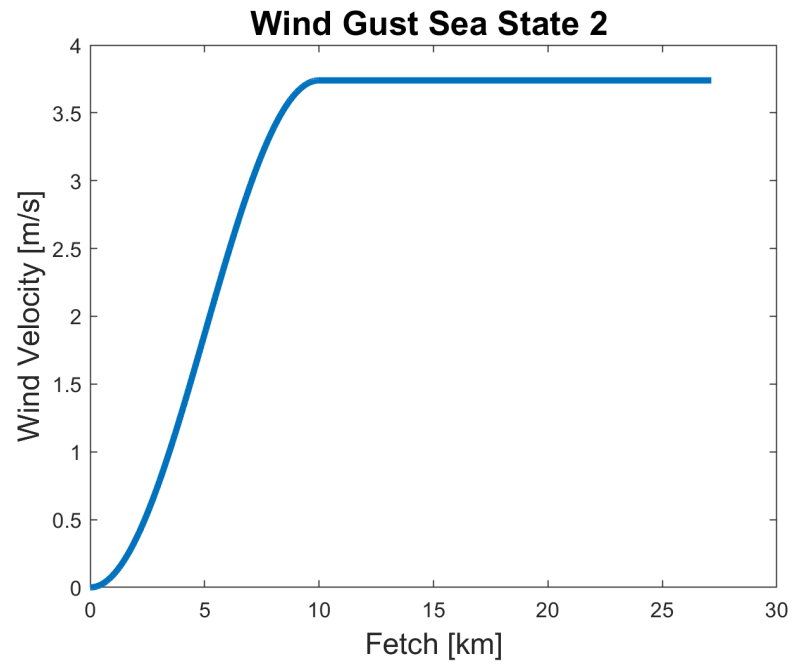

Figure 3.11: 1-cosine shape wind gust with a peak velocity reached at a fetch length of $10 \mathrm{~km}$. 
the simulation resulting in maintained gusts for the duration of the simulation. The direction of the wind gust correlates to the heading of the ship, where a heading of $0^{\circ}$ would be a longitudinal tail wind and a heading of $90^{\circ}$ is a lateral cross wind. Other headings not multiples of $90^{\circ}$ contain longitudinal and lateral components. Atmospheric turbulence is added to the wind gust to complete the wind profile.

\subsubsection{Atmospheric Turbulence}

Similar to wind gusts, turbulence is modeled as a spatially varying process and relies on the magnitude of the aircraft's velocity $\left|\vec{V}_{U A V}\right|$ through a frozen turbulence field. Turbulence is modeled by adding Gaussian white noise to an appropriate shaping filter in accordance to MIL-F-8785C [74]. The deterministic variables for shaping Gaussian noise are the turbulence spatial frequency $\Omega_{t}$, the aircraft's ground speed

velocity $\left|\vec{V}_{U A V}\right|$, the turbulence intensity $\sigma_{i}$ and the turbulence scale lengths $l_{i}$, where $i$ is either a longitudinal $u$, lateral $v$ or vertical $w$ component.

For low altitude flight, under $1000 \mathrm{ft}$, the vertical turbulence length scale $l_{w}$ is defined as the UAV's altitude $z_{U A V}$

$$
l_{w}=z_{U A V},
$$

and the longitudinal $l_{u}$ and lateral $l_{v}$ turbulence length scale's are functions of the UAVs altitude, such that

$$
l_{u}=l_{v}=\frac{z_{U A V}}{\left(0.177+0.000823 z_{U A V}\right)^{1.2}} .
$$

The turbulence intensities are calculated based on wind speed at $19.5 \mathrm{ft}(6 \mathrm{~m})$. The longitudinal intensity $\sigma_{u}$ and lateral intensity $\sigma_{v}$ are a function of the vertical turbulence intensity, where the vertical turbulence intensity is given by 


$$
\sigma_{w}=0.1 U_{19.5}
$$

and longitudinal and lateral intensities by

$$
\frac{\sigma_{u}}{\sigma_{w}}=\frac{\sigma_{v}}{\sigma_{w}}=\frac{1}{\left(0.177+0.000823 z_{U A V}\right)^{0.4}}
$$

The temporal frequency $\omega_{t}$ experienced by the aircraft in a turbulence field is found by multiplying the turbulence field's spatial frequency $\Omega_{t}$ by aircraft's velocity $\left|\vec{V}_{U A V}\right|$

$$
\omega_{t}=\left|\vec{V}_{U A V}\right| \Omega_{t}
$$

According to MIL-F-8785C, the velocity spectra in the longitudinal $\Phi_{u}\left(\omega_{t}\right)$, lateral $\Phi_{v}\left(\omega_{t}\right)$ and vertical $\Phi_{w}\left(\omega_{t}\right)$ directions are

$$
\begin{gathered}
\Phi_{u}\left(\omega_{t}\right)=\frac{2 \sigma_{u}^{2} l_{u}}{\pi\left|\vec{V}_{U A V}\right|} \frac{1}{1+\left(l_{u} \frac{\omega_{t}}{\left|\vec{V}_{U A V}\right|}\right)^{2}}, \\
\Phi_{v}\left(\omega_{t}\right)=\frac{\sigma_{v}^{2} l_{v}}{\pi\left|\vec{V}_{U A V}\right|} \frac{1+3\left(l_{v} \frac{\omega_{t}}{\left|\vec{V}_{U A V}\right|}\right)^{2}}{\left.1+\left(l_{v} \frac{\omega_{t}}{\left|\vec{V}_{U A V}\right|}\right)^{2}\right]^{2}}, \\
\Phi_{w}\left(\omega_{t}\right)=\frac{\sigma_{w}^{2} l_{w}}{\pi\left|\vec{V}_{U A V}\right|} \frac{1+3\left(l_{w} \frac{\omega_{t}}{\left|\vec{V}_{U A V}\right|}\right)^{2}}{\left[1+\left(l_{w} \frac{\omega_{t}}{\left|\vec{V}_{U A V}\right|}\right)^{2}\right]^{2}} .
\end{gathered}
$$

The square roots of spectral equations 3.38, 3.39 and 3.40 are used to derive the forming filters for which band limited white noise may be passed through to emulate turbulence. Matlab documentation provides the continuous Dryden turbulence transfer functions [75] for which white noise may be used to be 


$$
\begin{gathered}
H_{u}(s)=\sigma_{u} \sqrt{\frac{2 l_{u}}{\pi\left|\vec{V}_{U A V}\right|}} \frac{1}{1+\frac{l_{u}}{\left|\vec{V}_{U A V}\right|} s}, \\
H_{v}(s)=\sigma_{v} \sqrt{\frac{l_{v}}{\pi\left|\vec{V}_{U A V}\right|}} \frac{1+\frac{\sqrt{3} l_{v}}{\left|\vec{V}_{U A V}\right|} s}{\left(1+\frac{l_{v}}{\left|\vec{V}_{U A V}\right|} s\right)^{2}}, \\
H_{w}(s)=\sigma_{w} \sqrt{\frac{l_{w}}{\pi\left|\vec{V}_{U A V}\right|}} \frac{1+\frac{\sqrt{3} l_{w}}{\left|\vec{V}_{U A V}\right|} s}{\left(1+\frac{l_{w}}{\left|\vec{V}_{U A V}\right|} s\right)^{2}} .
\end{gathered}
$$

The transfer functions in equations $3.41,3.42$ and 3.43 give the atmospheric turbulence which is used in the model. The atmospheric turbulence is amalgamated with the wind gusts found in Section 3.4.1 forming the wind profile used to perturb the UAV. Figure 3.12 is an example of the amalgamated wind gusts and atmospheric turbulence the UAV would encounter when flying in sea state 6 with a wind direction of $60^{\circ}$. In this example, the lateral wind component dominates the longitudinal value due to the wind direction favoring the longitudinal access, the 1-cosine shape is observed in the first $10 \mathrm{~s}$. After UAV control is established in Section 3.5, Section 3.6 presents a study evaluating the UAV's trajectory tracking performance while flying through wind gusts and turbulence fields of various sea states. 


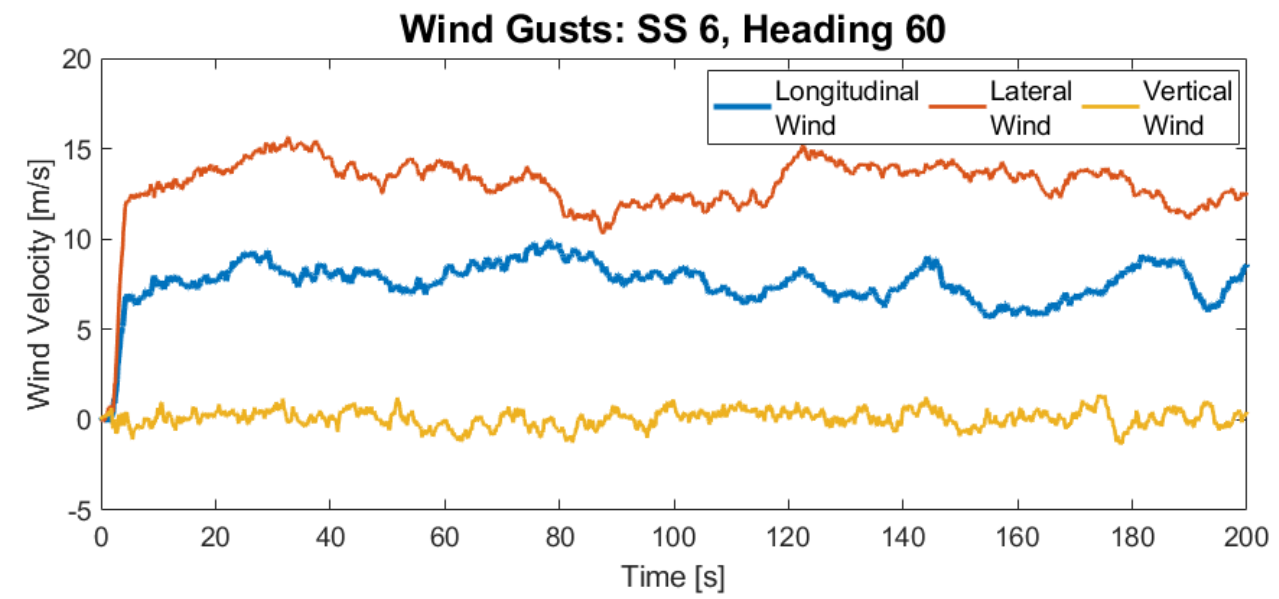

Figure 3.12: Atmospheric turbulence and wind gusts a UAV encounters when flying in sea state 6 with a wind direction of $60^{\circ}$

\subsection{UAV Control}

Proportion-Integral-Derivative (PID) controllers were used for the control and trajectory tracking of the QR UAV. PID controllers are a common control method for quad rotors and are used by companies such as DJI and PIX hawk [45]. Although nonlinear control techniques have been demonstrated to be effective [76-78], stable quad rotor control has been achieved with standard PID control $[43,44,79,80]$. In this thesis, satisfactory UAV control was achieved using the various PID tuning and control strategies outlined in this section. For model simplicity, the PID controllers are able to command bi-directional control signals, for example a positive roll command has a positive control output signal, where a negative roll command would have a negative control signal. Alternatively a non-zero bias threshold could have been set for direction control. A controller mixer is used to determine the aggregate of the altitude, yaw, roll, and pitch control signals. The UAV controllers were developed sequentially where the altitude controller was the first controller developed, followed by the yaw controller for heading stability and finally roll and pitch controllers. Due 
to the symmetry of the QR along the $\mathrm{x}-\mathrm{y}$ axes the same control strategy is used for roll and pitch control.

\subsubsection{Altitude Control}

For altitude control, a PID controller with a feed-forward loop was compared to a standard PID controller. For the feed-forward controller, a constant feed forward term was implemented to mitigate the effects of motor windup by maintaining a minimum motor speed close to the propeller operating point, thus increasing the responsivity of the UAV during take off. The feed-forward controller was found to be more responsive to an initial step response which came at the cost of an increased settling time. During flight, the response of the feed-forward controller was comparable to that of the standard PID controller. The improvement in the response during take off was not found to justify the increased settling time and a standard PID controller was chosen for altitude modulation. Figure 3.13 illustrates the altitude PID controller where, the error between the reference altitude and the sensor altitude is compensated using the sum of the proportional gain $K_{p A L T}$, the integral of gain $K_{i A L T}$ and the derivative of gain $K_{d A L T}$. The control signal saturates at $85 \%$ to ensure power remains for roll and pitch control. The remaining $15 \%$ of the total UAV control effort was split between the roll and pitch controllers and was found to be sufficient for maintaining roll and pitch stability during ship tracking. 


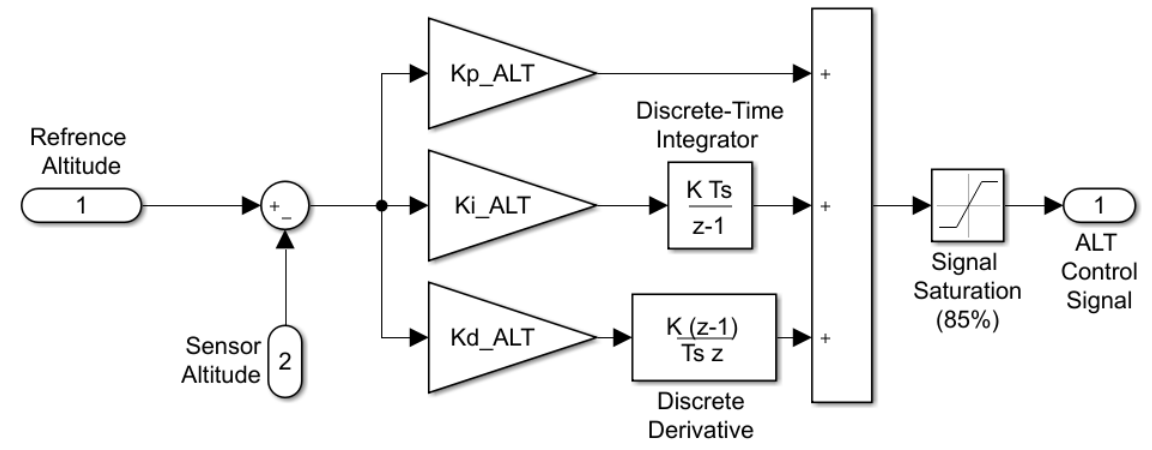

Figure 3.13: PID controller for UAV altitude.

All the controller's in this section were initially tuned using the Ziegler-Nicholas (Z-N) method [81]. Z-N tuning was performed by:

- Applying a step input to the controller

- The ultimate tuning proportional gain $K_{u}$ was increased until the system exhibited constant stable oscillations

- The oscillation period $T_{u}$ was then measured

- Using Z-N the PID controller and the associated gains having the following form

$$
u(s)=K_{p}\left(1+\frac{1}{T_{i} s}+T_{d} s\right) e(s)
$$

where,

$$
\begin{gathered}
K_{p}=0.6 K_{u}, \\
T_{i}=\frac{T_{u}}{2}, \\
T_{d}=\frac{T_{u}}{8} .
\end{gathered}
$$

Equation 3.44 may be expanded to solve for the integral gain $K_{i}$ and derivative gain 
$K_{d}$ as

$$
\begin{gathered}
K_{i}=\frac{K_{p}}{T_{i}}, \\
K_{d}=K_{p} T_{d} .
\end{gathered}
$$

Matlab Simulink's PID control system designer was then used to improve the altitude controller for a $1 \mathrm{~m}$ step input. Figure 3.14 illustrates the altitude PID controller's response, where the dotted black line is the step input, and the solid orange line is the UAV's response. The UAV exhibited a rise time of less than $1 \mathrm{~s}$, and settled within $2 \mathrm{~s}$. With linear control established on the z axis, yaw control was developed.

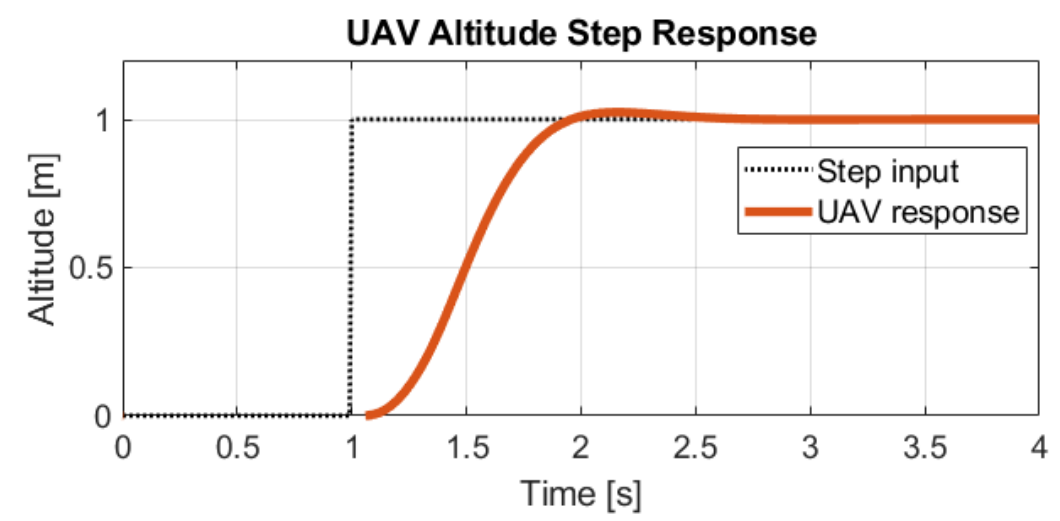

Figure 3.14: UAV altitude response to a step input of $1 \mathrm{~m}$.

\subsubsection{Yaw Control}

For yaw control, a standard PID controller was compared to a cascade P-PI yaw controller and tested for disturbance rejection. The cascade controller's outer-loop was a P-controller which aimed to compensate for angular displacement error and the inner-loop was a PI-controller to compensate for discrepancies in angular rate and operated at twice the rate of the outer-loop. Figure 3.15 is the cascaded yaw controller where the first error signal is the difference of the reference yaw and the sensor yaw, and the second error signal is the difference between the amplified yaw error signal and the yaw rate measurement. The yaw controller was saturated at $30 \%$ which is roughly 
half the power required to maintain a stable hover; although the yaw controller does not alter the net thrust of the UAV, it needs to be saturated to ensure it does not cut power to any of the motors. As yaw of a UAV is fully actuated, meaning the UAV is able to adjust yaw by adjusting the RPM of the propellers without translating, a P-PI controller was found to adequately compensate for perturbations. Further, the innerloop's faster operating frequency allows for more robust disturbance rejection [47]. The cascaded system showed better disturbance rejection compared to a standard PID controller. The yaw controller gains were tuned using the Z-N approach, however additional iterative tuning was performed as the Z-N method was found to be less effective in tuning yaw. After heuristically tuning the yaw gains, Simulink's Control System Designer was used to adjust the control gains of the inner-loop for angular velocity steps of $15 \mathrm{deg} / \mathrm{s}$ and the outer-loop for step inputs of $1^{\circ}$. The controller was tuned for small step inputs since the yaw reference signal remains zero during the simulation, as $\mathrm{x}$ and $\mathrm{y}$ translation is achieved through roll and pitch. Therefore, all yaw error signals are from wind and induced aerodynamic loading. Figure 3.16 highlights the disturbance rejection qualities of the P-PI controller compared to the standard PID, where the dotted black line is the reference signal, the P-PI controller is the solid orange line and the dashed yellow line is the PID controller. Gaussian noise with a bias of $2 \mathrm{~N}$ was applied in the $\mathrm{y}$ direction on the end of the UAV arm applying a moment about the UAV's CG. The P-PI controller was less disturbed and displayed quicker set-point convergence with less fluctuation from the set-point than the PID controller. With full actuation and control for the $\mathrm{z}$ axis, roll and pitch control can be developed. 


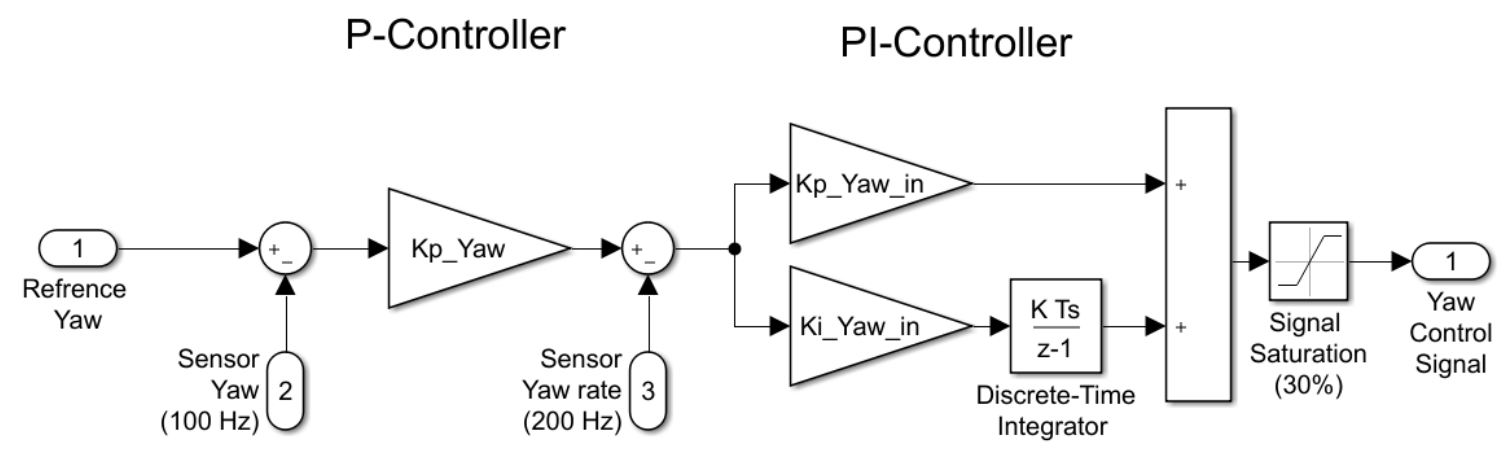

Figure 3.15: P-PI control strategy for UAV yaw.

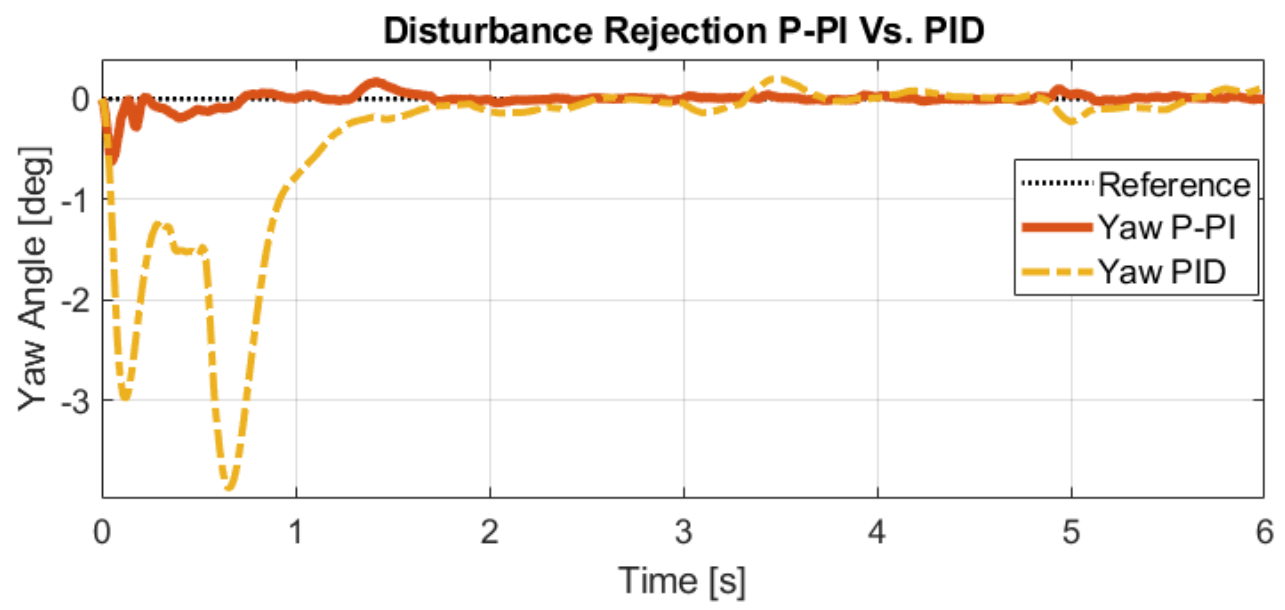

Figure 3.16: Yaw P-PI controller vs. PID controller disturbance rejection to a load applied on the UAV arm, resulting in a $0.7 \mathrm{Nm}$ moment about the CG.

\subsubsection{Roll and Pitch Control}

A PI-PID cascade controller, Figure 3.17, was found to be effective for accurately positioning the body angle while also rejecting wind perturbations. Similar to the yaw controller, the outer-loop PI-controller compensates for roll error and the inner-loop PID controller compensates the angular velocity differential between the outer-loop controller and the measured angular rate of the UAV. Again, the inner loop operates at $200 \mathrm{~Hz}$, twice the rate of the PI controller, thus compensating for disturbances without the need of the outer-loop to alter its signal. The outer-loop control signal is 
saturated at $60 \mathrm{deg} / \mathrm{s}$ to reduce angular acceleration. The first subplot of Figure 3.18 highlights the response of the roll controller for a $15^{\circ}$ step command, where the dotted black line is the reference signal and the solid orange line is the UAV's response. The UAV has a rise time of under $0.5 \mathrm{~s}$ with under $20 \%$ overshoot and a settling time of 2 s. The second subplot in Figure 3.17 highlights the inner-loops response to the control signal produced by the outer-loop, with the reference signal displayed as a solid blue line. The response is adequate when considering an instantaneous response is unfeasible due to rotational inertia. Further, the response shows quick convergence to the control subsequent to the step input. Due to the symmetry of the UAV, the same control strategy is also implemented for pitch control. The roll and pitch controllers complete the control system for fully actuating the UAV, however auxiliary controllers are required for trajectory tracking and positioning of the UAV.

PI-Controller

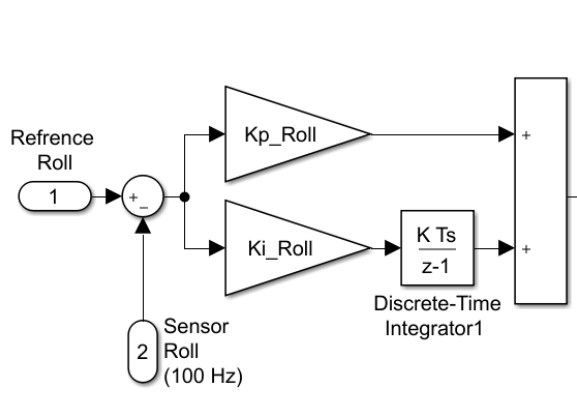

PID-Controller

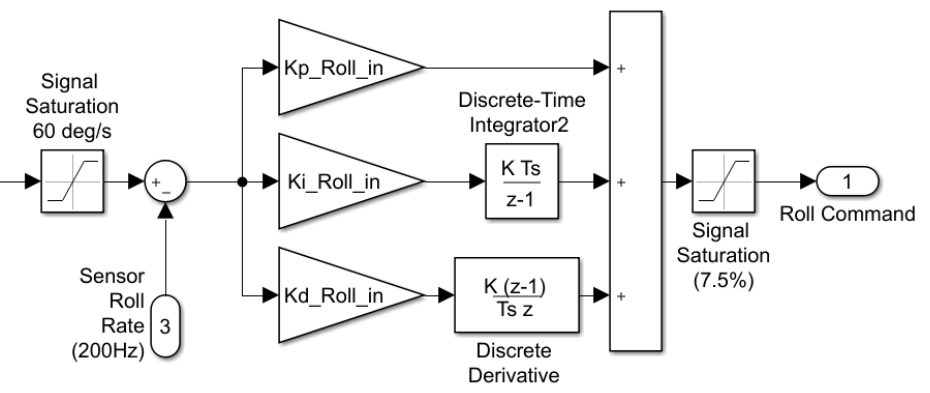

Figure 3.17: Cascade PI-PID control strategy for UAV roll and pitch controllers. 

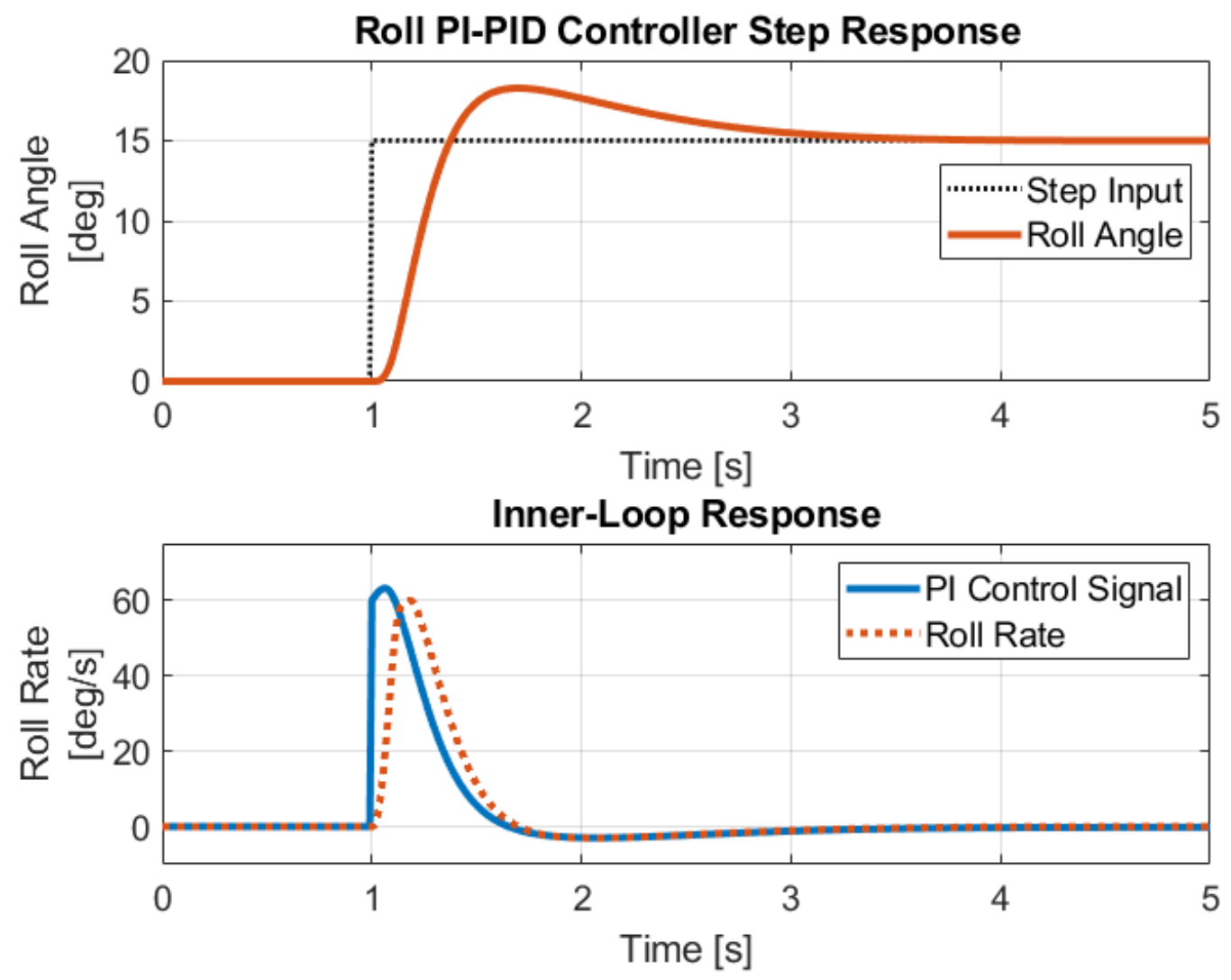

Figure 3.18: Outer and inner-loop response of a UAV roll controller subjected to a $15^{\circ}$ step input.

\subsubsection{Trajectory Tracking Control}

Currently, the UAV traverses using reference angles for the roll and pitch controllers, however, it is more useful to use coordinate information to direct the UAV. Figure 3.19 shows the trajectory tracking controller that uses a modulated PD controller with a saturated integral term to command the PI-PID roll and pitch controllers. The Pgain in Figure 3.19 is modulated to decrease by up to 5 times its value if the UAV is over $5 \mathrm{~m}$ away from its target. The modulation of the P-gain is used to decrease overshoot when the error signal is high, but increase tracking performance when in close proximity to the reference signal. P-gain modulation is implemented through a saturation block on the error term, with saturation values of 1 and 5 . The original 
P-gain is divided by the saturated error signal, thus modulating its gain to increase when the error is low and decrease when the error is high. An integral controller (Icontroller) with a saturation block, to prevent controller windup, is used to decrease steady state error which occurs during head wind conditions. Finally, a saturation block limits the maximum roll and pitch angles where a saturation of $25^{\circ}$ is found to keep the UAV stable in single axis flight, and saturation values of $20^{\circ}$ are used for $\mathrm{x}$ and y traversal. Saturation values of $20^{\circ}$ roll and $25^{\circ}$ pitch provided satisfactory results for ship tracking. Table 3.4 outlines the UAV gains used for UAV control and trajectory tracking.

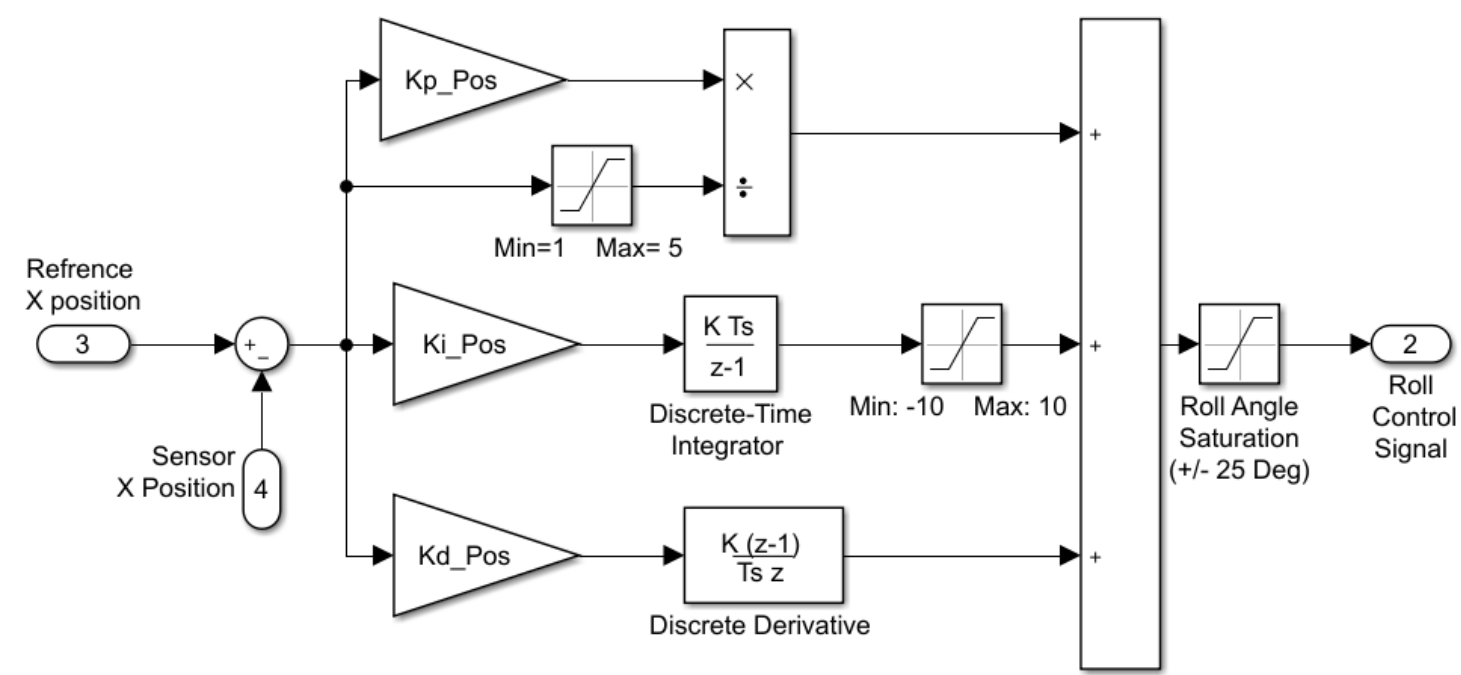

Figure 3.19: Control strategy for the UAV trajectory tracking controllers. 
Table 3.4: PID gains used for UAV control and trajectory tracking.

\begin{tabular}{|c|c|c|c|c|c|}
\hline Controller & \multicolumn{5}{|c|}{ Gains } \\
\hline \multirow{2}{*}{ Altitude } & P-Gain & I-Gain & D-Gain & & \\
\hline & 97.5 & 96.4 & 27.1 & & \\
\hline \multirow{3}{*}{ Yaw } & Outer-Loop & \multicolumn{2}{|c|}{ Inner-Loop } & & \\
\hline & P-Gain & P-Gain & PI-Gain & & \\
\hline & 9.75 & 2.1 & 45.6 & & \\
\hline \multirow{3}{*}{$\begin{array}{c}\text { Roll \& } \\
\text { Pitch }\end{array}$} & \multicolumn{2}{|c|}{ Outer-Loop } & \multicolumn{3}{|c|}{ Inner-Loop } \\
\hline & P-Gain & I-Gain & P-Gain & I-Gain & D-Gain \\
\hline & 8.25 & 3 & 0.5 & 0.75 & 0.025 \\
\hline & P-Gain & I-Gain & D-Gain & & \\
\hline $\begin{array}{c}\text { Trajectory } \\
\text { Tracking }\end{array}$ & 10.3 & 1 & 8.1 & & \\
\hline
\end{tabular}

Figure 3.20 is the UAV's response to a reference signal of $50 \mathrm{~m}$, where the blue line is the step input and the orange dotted line is the $\mathrm{x}$ position of the UAV. The response follows a sigmoid trajectory, a result of the P-gain modulation. A series of trajectory tracking tests are presented in the following section to access the UAV's flying performance in wind conditions matching the simulated sea states as well as its ability to track a ship's landing zone.

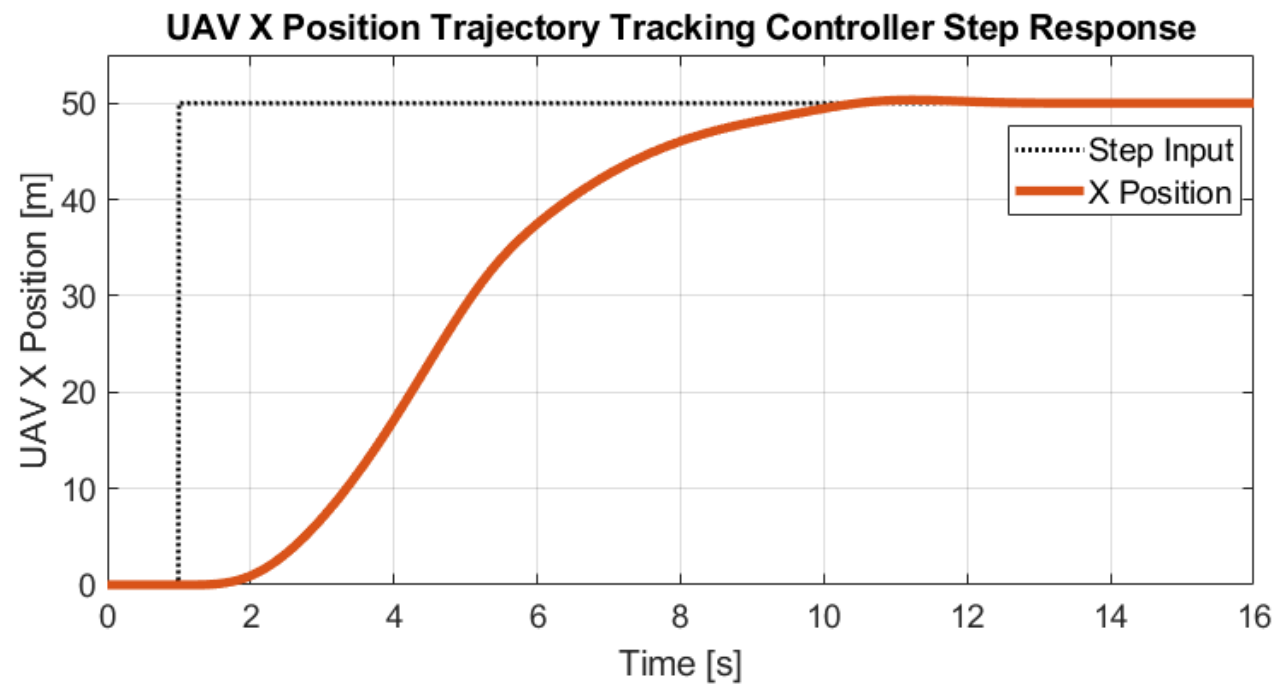

Figure 3.20: Trajectory tracking results for a $50 \mathrm{~m}$ step input. 


\subsection{Trajectory Tracking Results}

To evaluate the performance of the overall control structure with wind conditions the UAV performed a series of trajectory tracking tests. For the first series of tests, the UAV was to follow a spiral trajectory while wind gusts and atmospheric turbulence of the expected sea states perturbed the system. The UAV's starting position was with its $\mathrm{x}$-axis in line with north and y-axis with east. A spiral reference trajectory was formed by using two sinusoidal signals as reference inputs for the trajectory tracking controllers, where both signals had amplitudes and frequencies of $50 \mathrm{~m}$ and $0.1 \mathrm{rad} / \mathrm{s}$ respectively, and the $\mathrm{x}$ sinusoidal reference had a phase shift of $\pi / 2$. Figure $3.21 \mathrm{a}$ is an example of the UAV successfully following the spiral trajectory, where the solid black line is the reference trajectory and the red dashed line is the UAV's position. Table 3.5 details the results of all the spiral trajectory test. In total, there were 20 test cases ranging from sea states $2-6$, with wind headings ranging from $0^{\circ}$ to $90^{\circ}$. The UAV showed good tracking in winds up to sea state 4, where the average Euclidean distance between the UAV and reference trajectory was approximately $1 \mathrm{~m}$. The RMSE between the UAV's $\mathrm{x}$ position and the reference $\mathrm{x}$ trajectory was found to be higher on average than the y positions. The increased RMSE for $\mathrm{x}$ is due to the large error signal, $50 \mathrm{~m}$, between the UAV's starting x position and that of the sinusoidal signal. Whereas the initial y error signal started at $0 \mathrm{~m}$, leading to better set point tracking.

The UAV displayed poor performance in sea state 5 and 6 wind conditions. The average Euclidean for all cases was high, furthermore, in case 14 the UAV lost complete control and was blown away. The tracking performance significantly decreased in sea state 6 , where two of the four UAV's crashed. From the UAVs that did not crash, the overall tracking performance severely diminished. Figure 3.21b outlines the results from case 13, where the wind was blowing at $13.2 \mathrm{~m} / \mathrm{s}$ from south to north. The UAV, the red dashed line, was unable to overcome the induced drag from 
wind leading to unsatisfactory trajectory tracking. Figure 3.21c demonstrates the trajectory tracking results from case 14 , the UAV was blown significantly off course, however did not tumble and crash.

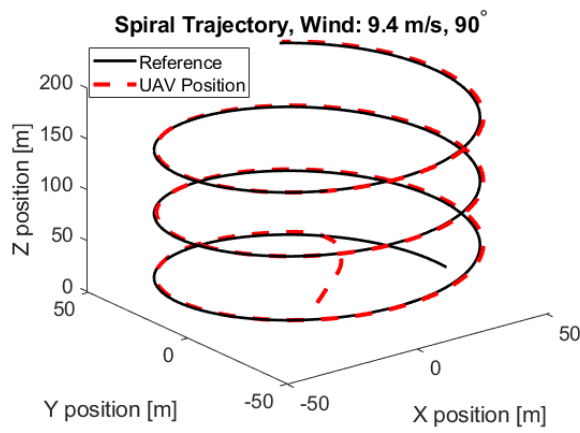

(a)

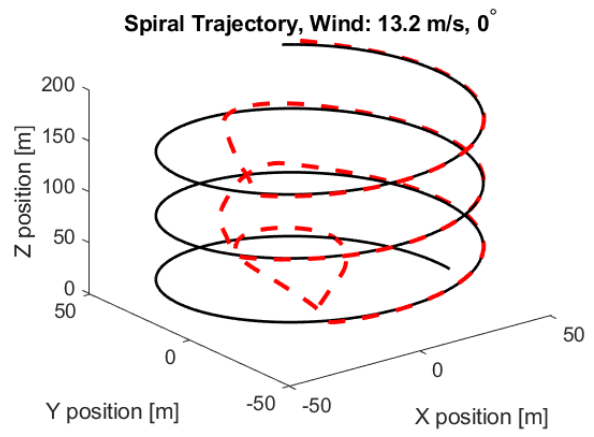

(b)

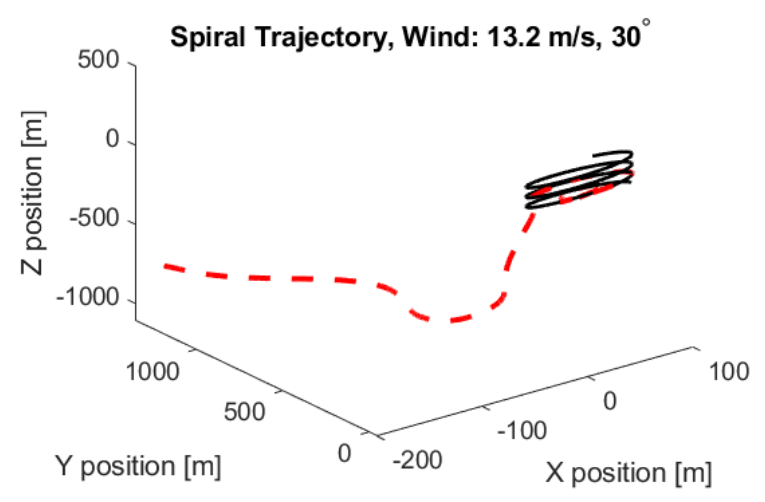

(c)

Figure 3.21: (a) Satisfactory UAV trajectory tracking of a spiral in sea state 4 winds with a wind direction of $90^{\circ}$. (b) Unsatisfactory trajectory tracking results of a UAV following a spiral in sea state 5 winds with a wind direction of $0^{\circ}$ (c)UAV unable to track spiral trajectory in sea state 5 winds blowing at $30^{\circ}$ 
Table 3.5: Spiral trajectory tracking results.

\begin{tabular}{|c|c|c|c|c|c|c|c|}
\hline Trial & $\begin{array}{c}\text { Sea } \\
\text { State }\end{array}$ & $\begin{array}{c}\text { Wind } \\
\text { Velocity } \\
{[\mathrm{m} / \mathrm{s}]}\end{array}$ & $\begin{array}{c}\text { Wind } \\
\text { Direction } \\
\text { [deg] }\end{array}$ & $\begin{array}{c}\text { RMSE } \\
\text { x } \\
{[\mathrm{m}]}\end{array}$ & $\begin{array}{c}\text { RMSE } \\
\text { y } \\
{[\mathrm{m}]}\end{array}$ & $\begin{array}{c}\text { RMSE } \\
\text { z } \\
{[\mathrm{m}]}\end{array}$ & $\begin{array}{c}\text { Avg. } \\
\text { Euclidean } \\
\text { Distance }[\mathrm{m}]\end{array}$ \\
\hline 1 & \multirow{4}{*}{2} & 3.75 & 0 & 1.06 & 0.18 & 0.002 & 1.03 \\
\hline 2 & & 3.75 & 30 & 1.06 & 0.20 & 0.002 & 1.04 \\
\hline 3 & & 3.75 & 60 & 1.07 & 0.20 & 0.002 & 1.07 \\
\hline 4 & & 3.75 & 90 & 1.05 & 0.19 & 0.002 & 1.04 \\
\hline 5 & \multirow{4}{*}{3} & 6.40 & 0 & 1.05 & 0.20 & 0.004 & 0.89 \\
\hline 6 & & 6.40 & 30 & 1.03 & 0.25 & 0.004 & 0.91 \\
\hline 7 & & 6.40 & 60 & 1.03 & 0.27 & 0.004 & 0.99 \\
\hline 8 & & 6.40 & 90 & 1.05 & 0.23 & 0.004 & 1.05 \\
\hline 9 & \multirow{4}{*}{4} & 9.40 & 0 & 0.85 & 0.23 & 0.008 & 0.75 \\
\hline 10 & & 9.40 & 30 & 0.83 & 0.33 & 0.008 & 0.79 \\
\hline 11 & & 9.40 & 60 & 1.01 & 0.43 & 0.008 & 0.94 \\
\hline 12 & & 9.40 & 90 & 1.06 & 0.34 & 0.007 & 1.09 \\
\hline 13 & \multirow{4}{*}{5} & 13.20 & 0 & 9.86 & 0.33 & 0.018 & 5.32 \\
\hline 14 & & 13.20 & 30 & 102.68 & 908.96 & 1024.687 & 1286.11 \\
\hline 15 & & 13.20 & 60 & 0.67 & 7.71 & 0.064 & 4.26 \\
\hline 16 & & 13.20 & 90 & 1.07 & 5.21 & 0.023 & 3.47 \\
\hline 17 & \multirow{4}{*}{6} & 15.30 & 0 & 42.29 & 0.36 & 0.021 & 29.55 \\
\hline 18 & & 15.30 & 30 & * & $*$ & $*$ & $*$ \\
\hline 19 & & 15.30 & 60 & $*$ & $*$ & $*$ & $*$ \\
\hline 20 & & 15.30 & 90 & 1.04 & 30.86 & 0.025 & 22.69 \\
\hline
\end{tabular}

The second series of trajectory tests was performed to evaluate how well the UAV could track the landing zone while in a low hover during sea state 6 motion with winds blowing at $10 \mathrm{~m} / \mathrm{s}$. Wind gusts in the model were saturated at $10 \mathrm{~m} / \mathrm{s}$ due to 
the UAV's maximum True Air Speed (TAS) limit, which was found to be $17 \mathrm{~m} / \mathrm{s}$ in Table 3.2. The maximum ship velocity in the simulation was $10 \mathrm{kn}(5.1 \mathrm{~m} / \mathrm{s})$; using Equation 3.14 the maximum head wind a UAV can fly in and still track the ship is $17-5.1=11 \mathrm{~m} / \mathrm{s}$, however, the UAV must also be able to travel faster than ship in order to track it. A wind velocity of $10 \mathrm{~m} / \mathrm{s}$ was found to be the upper limit for reaching and tracking the ship.

Figure 3.22 is an example of the UAV starting configurations for all ship tracking and landing simulations. The UAV's coordinate frame is always set with the $\mathrm{x}$-axis inline with the ship's velocity vector and takes off $50 \mathrm{~m}$ behind and $10 \mathrm{~m}$ to the port side of the ship.

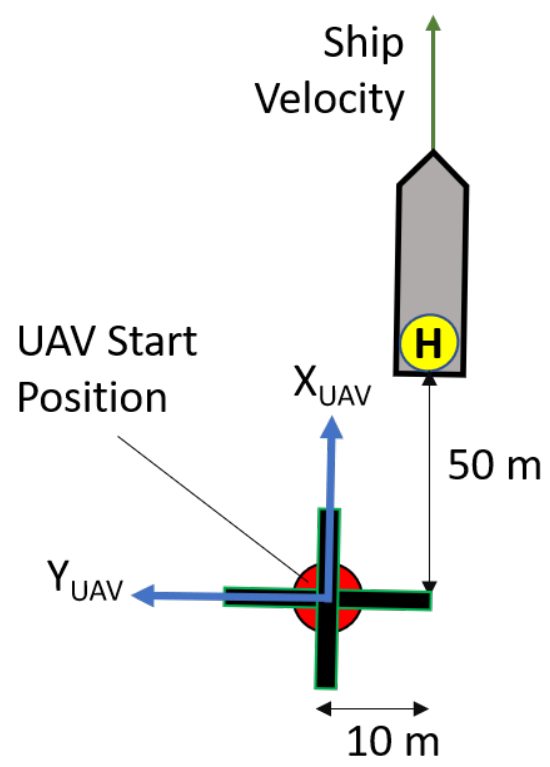

Figure 3.22: Starting position of UAV during simulations

At the start of the simulation the UAV climbs to its cruising altitude of $15 \mathrm{~m}$, tracks the ship, and descends to a low hover position of $5 \mathrm{~m}$ above the ship deck. Once the UAV descends from its cruising altitude to its low hover position the tracking results were recorded for the remainder of the $600 \mathrm{~s}$ simulation. Seven ship tracking tests were performed with ship headings ranging from $0^{\circ}$ to $180^{\circ}$ in increments of $30^{\circ}$. Figure 3.23 shows the XYZ tracking error results for Trial 3. A black circle highlights 
the $\mathrm{X}-\mathrm{Y}$ bounds of the landing zone and the error is plotted using a coloured line with a matching simulation time scale. Table 3.6 highlights the results for the 7 tracking conditions. The results obtained from this set of data ensure that the UAV is able to track the landing zone at the most volatile ship motion in wind conditions not exceeding $10 \mathrm{~m} / \mathrm{s}$. The UAV was able to track the ship motion with an average Euclidean distance less than $1.15 \mathrm{~m}$. In order to safely land on the ship, the ship motion and landing condition criteria must first be examined.

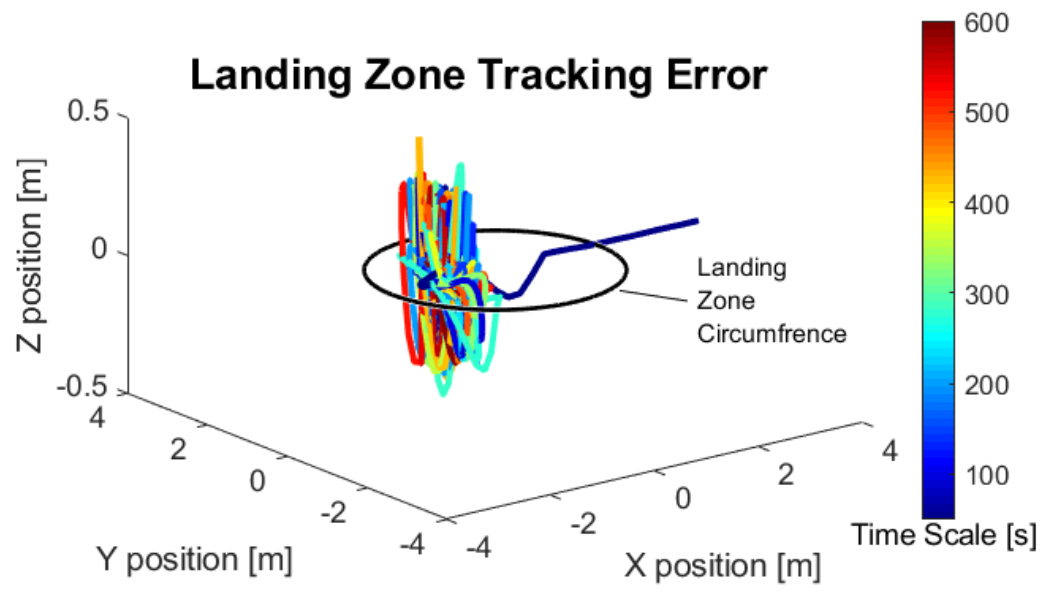

Figure 3.23: Tracking error between UAV and ship landing zone while maintaining a low hover of $5 \mathrm{~m}$.

Table 3.6: UAV ship tracking error for sea state 6 ship motion with winds of $10 \mathrm{~m} / \mathrm{s}$.

\begin{tabular}{|c|c|c|c|c|c|c|c|c|}
\hline Trial & $\begin{array}{c}\text { Ship Heading \& } \\
\text { Wind direction [deg] }\end{array}$ & $\begin{array}{c}\text { RMSE x } \\
\text { Distance [m] }\end{array}$ & $\begin{array}{c}\text { RMSE y } \\
\text { Distance [m] }\end{array}$ & $\begin{array}{c}\text { RMSE z } \\
\text { Distance }[\mathrm{m}]\end{array}$ & $\begin{array}{c}\text { Avg. Euclidean } \\
\text { Dist. [m] }\end{array}$ & $\begin{array}{c}\text { RMS Drag } \\
\text { Force x [N] }\end{array}$ & $\begin{array}{c}\text { RMS Drag } \\
\text { Force y [N] }\end{array}$ & $\begin{array}{c}\text { RMS Darag } \\
\text { Force z [N] }\end{array}$ \\
\hline 1 & 0 & 1.13 & 0.24 & 0.10 & 1.15 & 0.40 & 0.07 & 0.31 \\
\hline 2 & 30 & 1.12 & 0.27 & 0.09 & 1.16 & 0.37 & 0.71 & 0.47 \\
\hline 3 & 60 & 0.99 & 0.33 & 0.09 & 1.04 & 0.17 & 1.32 & 0.67 \\
\hline 4 & 90 & 0.69 & 0.28 & 0.07 & 0.74 & 1.20 & 2.07 & 1.04 \\
\hline 5 & 120 & 0.21 & 0.21 & 0.03 & 0.26 & 3.24 & 2.57 & 1.87 \\
\hline 6 & 150 & 0.39 & 0.19 & 0.02 & 0.38 & 4.03 & 1.29 & 2.30 \\
\hline 7 & 180 & 0.39 & 0.16 & 0.01 & 0.36 & 4.61 & 0.24 & 2.29 \\
\hline
\end{tabular}




\subsection{Ship Motion}

In this thesis the ship motion was created in ShipMo3D [22] and imported into the Simulink model. In the Simulink model wind and sea waves always flow south to north. Ship heading is illustrated in Figure 3.24 where on the left the ship has a heading of $30^{\circ}$ degrees north, and on the right a heading of $120^{\circ}$ north. Table 5.2 highlights the ship motion created using ShipMo3D. The ship was simulated in 5 sea states at 3 velocities and 7 heading angles, totaling 105 sets of ship motion. Ship headings which align closer to the north or south pole, Figure 3.24a, predominately face incoming or outgoing waves, thus increasing the pitch motion experienced by the ship. Lateral headings closer to the east or west poles, Figure 3.24b, increase the angle between the bow and incoming waves leading to higher roll motion.

Table 3.7: Ship motion cases created in ShipMo3D used in UAV-ship simulator.

\begin{tabular}{|l|l|}
\hline Ship motion Parameters & Range \\
\hline Sea State & 23456 \\
\hline Ship Velocity (kn) & 6810 \\
\hline Ship Heading (deg) & 0306090120150180 \\
\hline Total & 105 \\
\hline
\end{tabular}

Sea state, ship velocity, and wave direction vastly alter the ship's motion as it traverses through a sea. In higher sea states, the accessibility to safe landing times is often difficult to come across as well as short lived. The primary objective of this thesis is to land the UAV when the absolute value of the ship's roll and pitch are less than $5^{\circ}$ and $2^{\circ}$, respectively. When the ship's motion is below the roll and pitch thresholds it is deemed a Go condition, which is a green light for landing. If the ship motion is outside the threshold that is a NoGo condition meaning it is not safe to land. Figure 3.25 is an illustrative example of how the Go-NoGo conditions are 


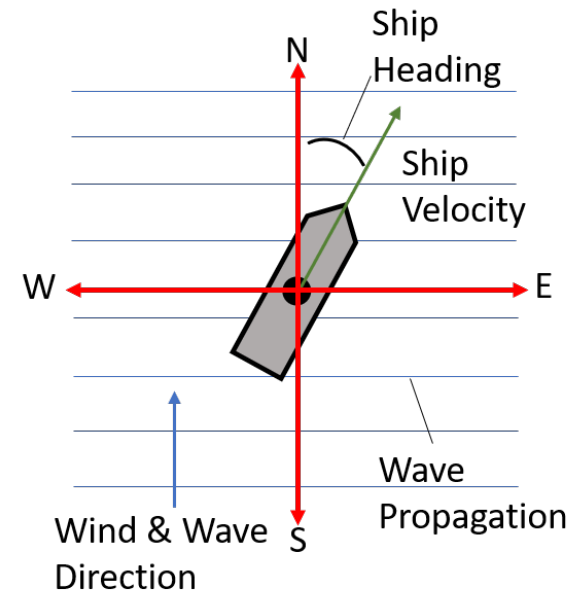

(a)

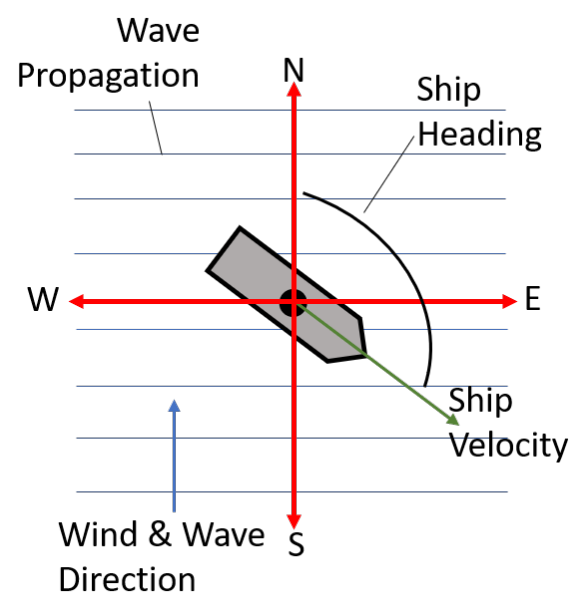

(b)

Figure 3.24: (a) $30^{\circ}$ ship heading,(b) $120^{\circ}$ ship heading.

defined with respect to the ship's roll and pitch motions. The first subplot in Figure 3.25 outlines the ship's roll motion and the corresponding threshold of $5^{\circ}$. For this set of ship motion roll never exceeds the $\pm 5^{\circ}$ thresholds. The second subplot is the pitch motion and its threshold of $\pm 2^{\circ}$. In the third subplot of Figure 3.25 the Go timing for when the roll and pitch are within their thresholds is indicated by a high signal. Sustained Go periods lasting at least $5 \mathrm{~s}$ are shaded in red and represent possible landing opportunities for the UAV. 


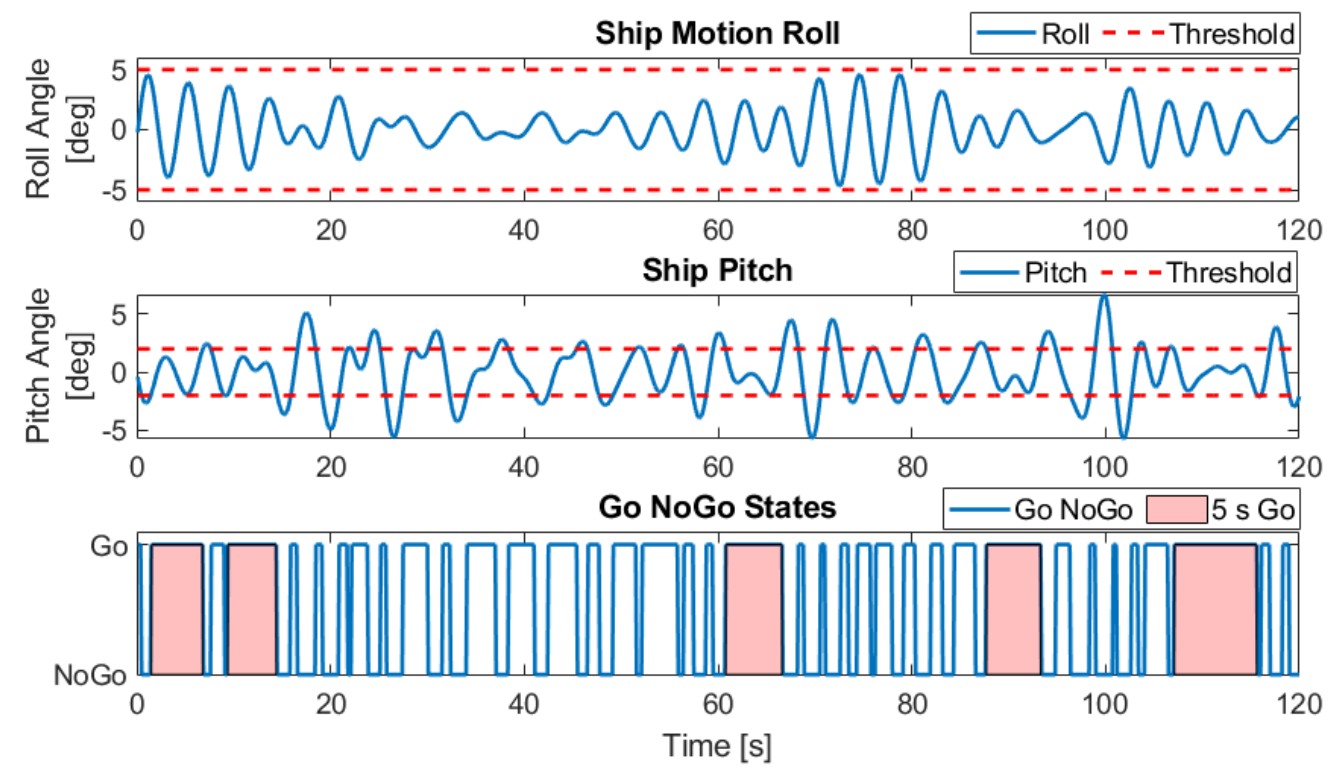

Figure 3.25: Roll and pitch angles of a ship travelling in sea state 3 with a $15^{\circ}$ heading and corresponding Go NoGo states.

Figure 3.26 outlines the RMS of roll, pitch and heave positions and velocities for a ship traversing in sea state 4 at $8 \mathrm{kn}$ as a function of the ship's heading into the sea, where the solid orange lines indicate position, and the dashed blue lines indicate velocity. The inverse relationship between roll and pitch is observed, where pitch motion is greater when the headings are closer to $0^{\circ}$ or $180^{\circ}$ and roll motion peaks the closer the heading is to $90^{\circ}$. The heave of the ship is strongly coupled with the pitch of the ship. Heading's towards the north $\left(0^{\circ}\right)$ leads to increased pitch and heave motion from the ship riding the waves, however when travelling south, ship-wave collisions dampen the pitch and heave motion. Higher sea states amplify the ship motion, thus a UAV has a significantly higher chance of landing successfully on ship travelling in lower sea states. 

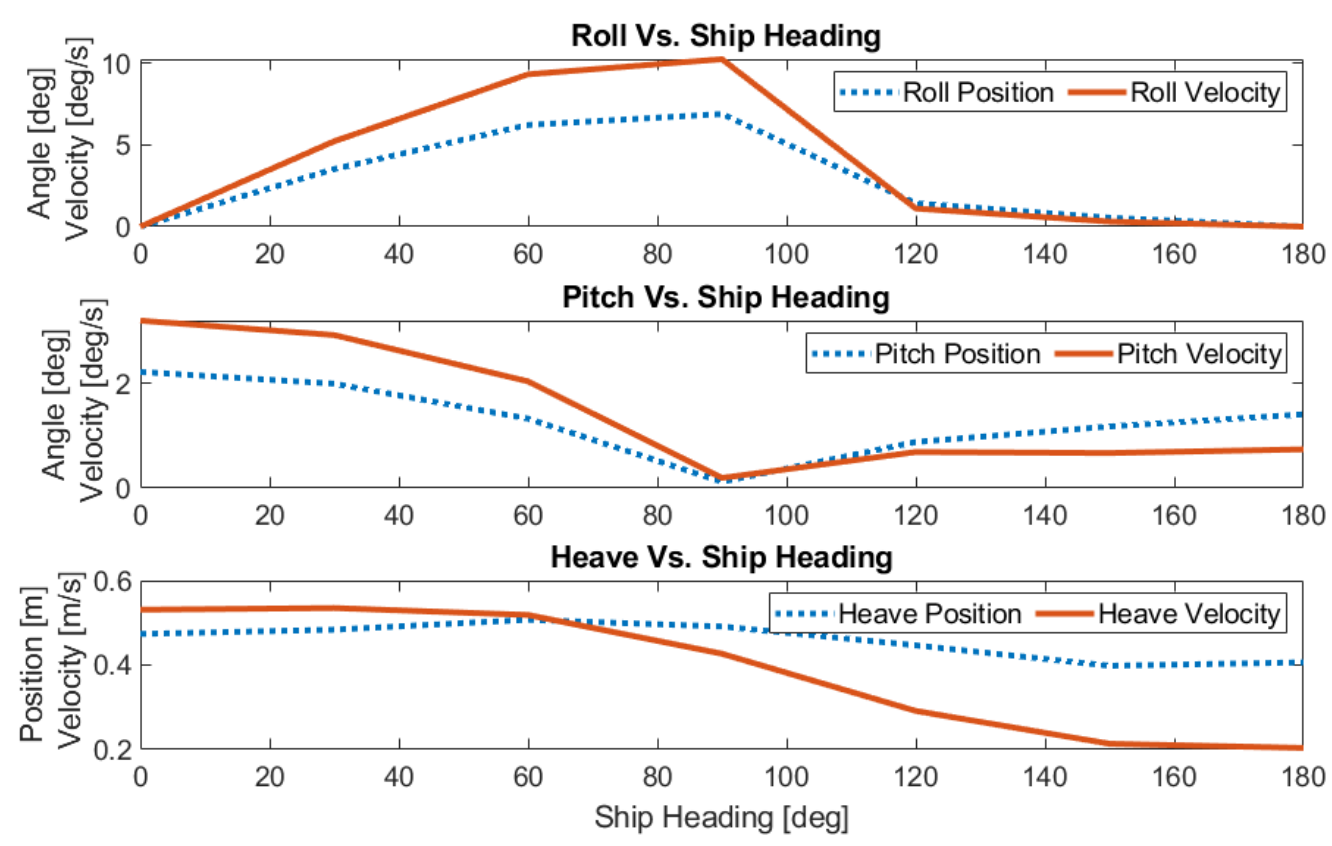

Figure 3.26: Ship heading angle influence on ship motion during sea state 4 .

To quantify the UAV's accessibility to safe landing times for the ship motion used in this thesis, Table 3.8 outlines the average time a Go period lasts for $5 \mathrm{~s}$ or longer for each set of sea state data. There is a significant drop in the amount of safe landing time as the sea states progress. As indicated by the increasing standard deviation, there is increased variability in the amount of sustained Go time at higher sea states, foreshadowing that some sets of ship motion have an increased risk of an unsuccessful landing due to the lack of landing opportunities. There are 3 instances in sea state 5 where there is never a suitable time to land, and 2 instances in sea state 6 . The absence of adequate Go time will hinder the UAV's landing performance as later highlighted in Section 5. In order to detect ship motion for landing, the UAV uses three on-board LIDARs and a ship pose detection algorithm. 
Table 3.8: Average Go time sustained for $5 s$ or more vs. sea state for simulated ship motion (21 sets of ship motion data per sea state).

\begin{tabular}{|c|c|c|c|c|c|}
\hline Sea State & 2 & 3 & 4 & 5 & 6 \\
\hline $\begin{array}{c}\text { Average Landing } \\
\text { Time >5 s }\end{array}$ & 600.0 & 479.5 & 279.4 & 141.0 & 140.1 \\
\hline Std. & 0 & 188.6 & 193.2 & 150.2 & 146.1 \\
\hline
\end{tabular}

\subsection{LIDAR and Ship Pose Detection}

The ability of an aircraft to independently determine the pose of a ship increases the cross-platform accessibility between the aircraft and ally ships. Current aerial systems may use time of flight instrumentation, such as LIDAR or SONAR to acquire ship pose information or optical instruments such as infrared and other digital cameras. Identifying ship orientation, without communication with the ship, may be achieved through coupling the UAV's Inertial Measurement Unit (IMU) with ranging or optical information.

In this research, 3 single element LIDARs were investigated to determine the feasibility of their use in determining ship pose. Carleton's Multi-Domain Lab owns multiple single entity LIDARs, Garmin LIDAR-Lite V1s [82], thus it would be beneficial to simulate existing hardware for later use. Further, ranging devices are less affected by lighting conditions and environmental visibility factors than their optical counterparts. By providing their own energy, LIDARs are unaffected by daylight visibility and are generally unimpaired by mist and fog [83]. If successful in simulation, the LIDAR-Lite may theoretically be used to construct an inexpensive aerial ship pose detection system for rotorcraft. 


\subsubsection{LIDAR Emulation}

The following section contains excerpts from a research paper published in proceedings of IEEE Oceans 18 Charleston titled Unmanned Aerial Vehicle Landing on Maritime Vessels using Signal Prediction of the Ship Motion [9]. Figure 3.27 illustrates the notation used in the following section to derive the output of each LIDAR. In order to simulate the LIDAR, the location of each LIDAR as well as the plane of the ship deck must be found. Three points $l_{i: 1,2,3}$ were placed under the UAV as a reference location for each LIDAR. Points $l_{i}$ are represented using position vectors $\vec{L}_{i}$ with respect to the global coordinate frame, where $i$ corresponds to either the $1^{\text {st }} 2^{\text {nd }}$ or $3^{r d}$ LIDAR. Using the cross product between vectors $\vec{L}_{i}$ the normal vector of the UAV is found by

$$
\vec{n}_{L}=\left(\vec{L}_{1}-\vec{L}_{2}\right) \times\left(\vec{L}_{1}-\vec{L}_{3}\right) .
$$

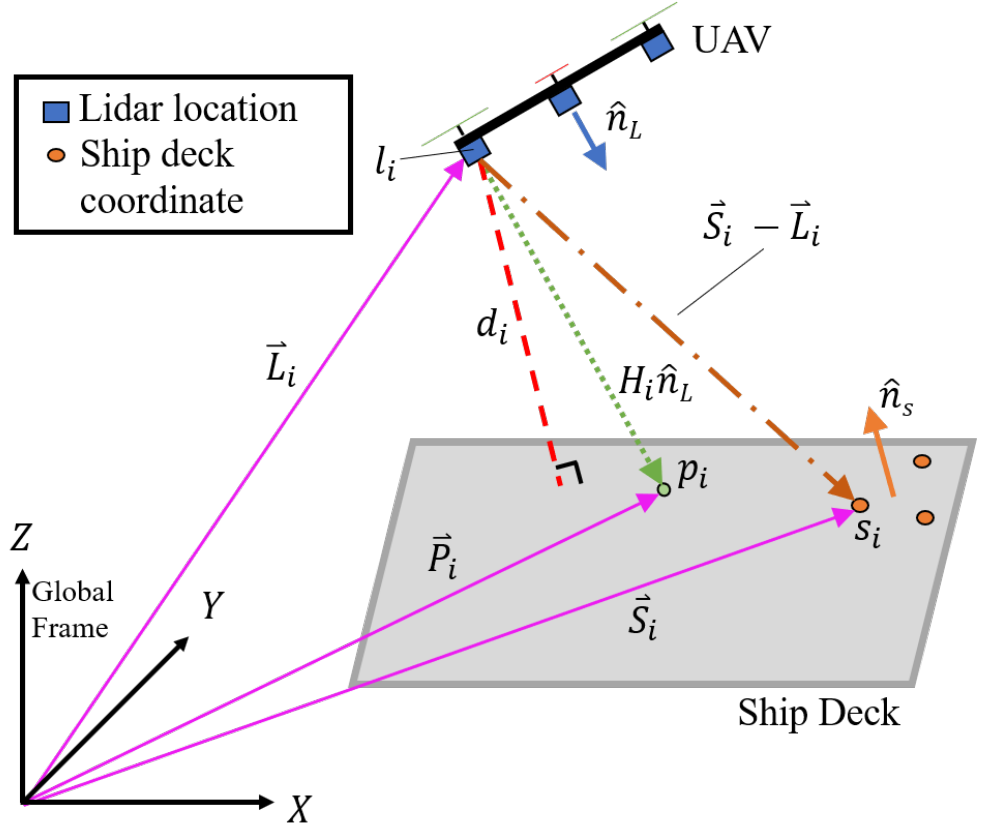

Figure 3.27: Schematic of UAV and the notation used to derive the simulated LIDAR outputs.

Vector $\vec{n}_{L}$ is normalized to form the UAV's unit normal vector $\hat{n}_{L}$. Similarly within 
the simulation, three points $s_{i: 1,2,3}$, and corresponding position vectors $\vec{S}_{i: 1,2,3}$, were placed on the deck of the ship. Using the cross product between position vectors $\vec{S}_{i}$ the normal vector of the ship is found and then normalized $\hat{n}_{s}$. Using the UAV and ship orientation, the simulated LIDAR outputs are found. Through vector addition, an equation which relates the magnitude and direction of the LIDAR output to its final and initial vectors is

$$
H_{i} \hat{n}_{L}=\vec{P}_{i}-\vec{L}_{i}
$$

where $H_{i}$ is the magnitude of the LIDAR output and $\vec{P}_{i}$ is the position vector of point $p_{i}$ where the LIDAR intersect the plane of the ship deck. The shortest distance between a LIDAR and the plane of the deck $d_{i}$ can be found by taking the dot product between the ship's normalized unit vector with any vector originating at the LIDAR and intersecting a known point on the ship's plane, thus

$$
d_{i}=\hat{n}_{S} \cdot\left(\vec{S}_{i}-\vec{L}_{i}\right)
$$

$d_{i}$ will always be perpendicular to the plane of the ship regardless of the pose of the UAV. The same offset may be expressed in terms of the unknown position vector $\vec{P}_{i}$ as

$$
d_{i}=\hat{n}_{S} \cdot\left(\vec{P}_{i}-\vec{L}_{i}\right)
$$

The magnitude of each LIDAR $H_{i}$ is then found by substituting Equation 3.51 into Equation 3.53,

$$
H_{i}=\frac{d_{i}}{\hat{n}_{s} \cdot \hat{n}_{L}}
$$

In practice, the physical LIDARs would output distance measurement $H_{i}$. Within 
the simulation, the magnitude of the LIDARs is found through the above process, and are used to determine the pose of the ship.

\subsubsection{Ship Pose Detection}

The LIDARs pass their magnitude information $H_{i}$ into a separate algorithm which calculates the ship pose based on variations in the LIDAR outputs and angular pose of the UAV. This ship pose algorithm would be implemented on a physical UAV to determine ship deck motion without the need for ship IMU information. Figure 3.28 illustrates the ship deck motion in relation to the UAV and notation used. The algorithm works by identifying the three points, $p_{U A V_{i}}$, where the lasers intersects the deck with respect to the UAV. Without any noise, the points of intersection will be equivalent to $p_{i}$ from the previous section, but will be found in the UAV's coordinate frame. Rotation of the UAV during flight alters the LIDARs directional vector which initially faces down $[0,0,-1]$. To properly orient the LIDAR vectors during UAV manoeuvres, the vectors are multiplied by a series of rotation matrices. Following the right hand rule, the UAV pitch, roll and yaw angles are taken from the UAV's IMU, where rotations about the x-axis are roll $\Phi_{U A V}$, rotations about the y-axis are pitch $\Theta_{U A V}$, and rotations about the $\mathrm{z}$ axis are yaw $\Psi_{U A V}$. The corresponding rotation matrices from equations 3.16, 3.17 and 3.18 are used to orient the LIDAR vectors in the UAV's coordinate frame. The position vectors for each LIDAR $P_{U A V_{i}}$ in the UAV's translating frame is

$$
\vec{P}_{U A V_{i}}=R_{z}\left(\Psi_{U A V}\right) R_{y}\left(\Theta_{U A V}\right) R_{x}\left(\Phi_{U A V}\right)\left(H_{i} \vec{c}+\vec{k}_{i}\right),
$$

where $H_{i} \vec{c}$ is the vector formed between the LIDARs magnitude and the original directional vector for each LIDAR, and $\vec{k}_{i}$ is the LIDARs positional offset from the UAV's centre of rotation. 
Similar to Equation 3.50, the unit normal vector of the ship $\hat{n}_{S}$ is found by normalizing the cross product between $\vec{P}_{U A V_{1}{ }_{3}}$. The first index of $\hat{n}_{S}$ is the ship's roll angle and the third index is the pitch angle. The ship's roll and pitch angles are computed in the UAV's non-rotating body frame and are therefore equivalent to the ship's pitch and roll in the global frame. These roll and pitch angles are subsequently fed to their respective SPA. Without LIDAR noise, the normalized unit vector will be equivalent to $\hat{n}_{s}$ found in the previous section. The addition of noise complicates the system and the potential of error increases.

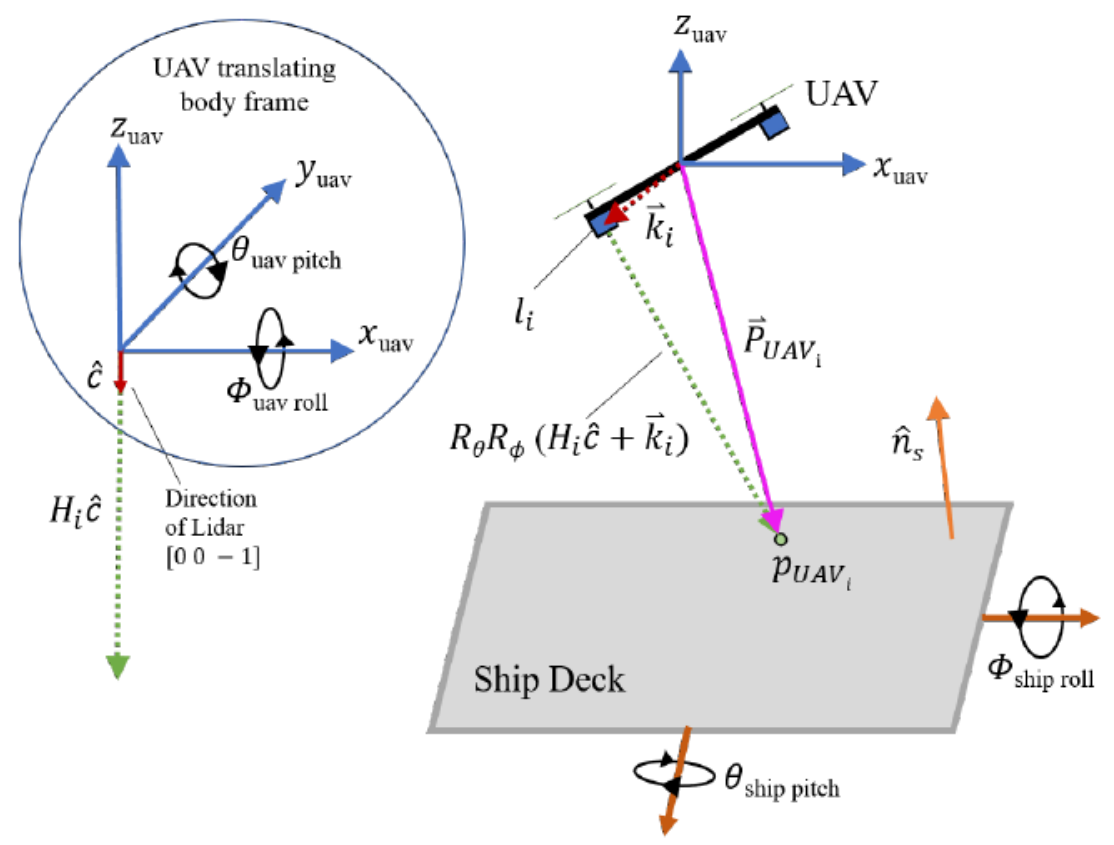

Figure 3.28: Ship pose detection illustrated notation.

\subsubsection{LIDAR Noise \& Filtering}

The Garmin LIDAR-Lite single pulse has a specified accuracy of $\pm 2.5 \mathrm{~cm}$. The LIDARs were initially simulated in idealized circumstances, however, Gaussian noise was added to the LIDAR signal. With added noise, discrepancies of $\pm 2.5 \mathrm{~cm}$ on each LIDAR results in large fluctuations in the calculated normal vector of the ship. Moreover, the closer the grouping of the LIDARs, the greater the associated error 
will be. Furthermore, the SPA will attempt to predict a noisy signal unless the input signal is filtered. Since noise cannot be assumed to be periodic, prediction error accumulates immediately; hence the noisier the signal, the more likely it is that the forecast signal will output false Go-NoGo states. It is therefore important that most noise in the LIDAR signals be filtered out before sending the signals to the SPA. Errors may be minimized by either changing the viewing angle of the LIDARs or spacing the LIDARs further apart. Changing the viewing angle of the LIDARs may be a solution if there is a large landing zone, however, a large viewing angle would increase the likelihood of the LIDAR pulse being intercepted by an object on the deck or potentially missing the deck all together. Depending on the size of the craft, or in this case, the length of the UAV arms, the LIDARs may be spaced out to decrease ship pose estimation error.

A sensitivity study was performed to evaluate the influence of LIDAR spacing and filtering. The study comprised of two parts, the first focused primarily on the effects of spacing and how much error could be mitigated by spreading the LIDARs apart. However, the length of the arms are finite and for that reason a second study was performed to evaluate the effectiveness of filtering the LIDARs. Figure 3.29 displays the RMSE of the roll and pitch values estimated by the UAV compared to the truth, where the spacing of the LIDARs ranged from $0.1 \mathrm{~m}$ to $5 \mathrm{~m}$. The second subplot displays how spacing affects the overall Signal-to-Noise Ratio (SNR). The set of ship motion used for this study was roll dominant and was less impacted by noise than pitch data due to the greater signal amplitude. Ideally, the grouping of LIDARs would be placed at least $2 \mathrm{~m}$ apart where the observed error is greatly reduced. On the UAV the maximum possible spacing of the LIDARs is $0.36 \mathrm{~m}$ (14 in) which has an associated RMSE of $2.5^{\circ}$ roll and $5^{\circ}$ pitch. Filtering strategies were tested to reduce the overall error for a LIDAR grouping spaced $0.36 \mathrm{~m}$ from the origin.

The second study compared the effects of filtering both the LIDAR signals and 

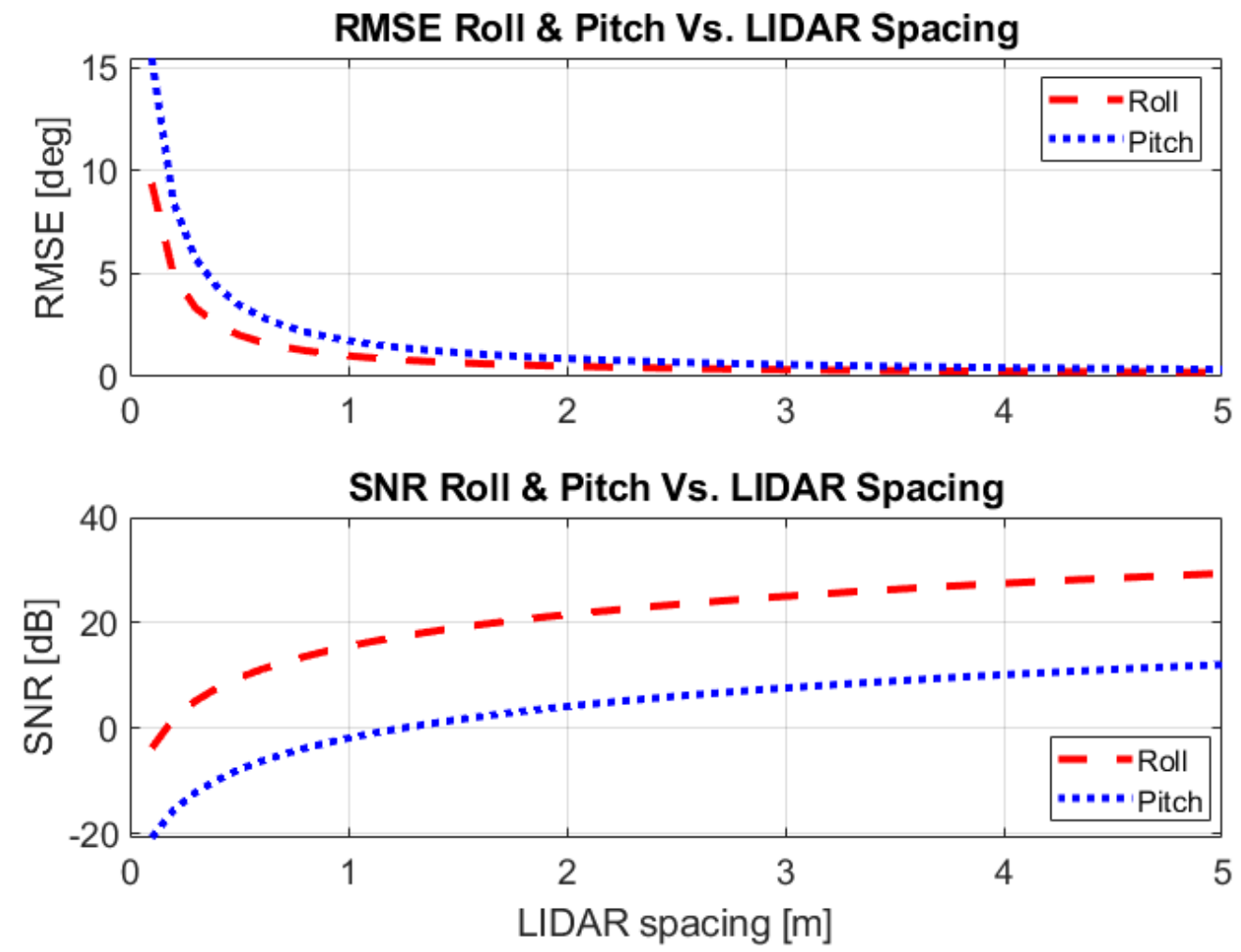

Figure 3.29: LIDAR spacing influence on gathered ship motion RMSE and SNR.

the estimated roll and pitch signals. In total 12 combinations of filters were compared and are highlighted in Table 3.9. The 3 filters used were a Kalman Filter (KF), a Moving Average Filter (MA), and an Exponential Moving Average Filter (EMA). A simple $\mathrm{KF}$ is used to smooth the initial LIDAR measurements by estimating the error and uncertainty in measured LIDAR signal. The KF is implemented in four steps. The first step initializes the system state $\hat{x}_{k}$ and error covariance $\epsilon_{k}$ by

$$
\begin{gathered}
\hat{x}_{k}=H_{k}^{-1} z_{k}, \\
\epsilon_{k}=H_{k}^{-1} R\left(H^{\top}\right)^{-1},
\end{gathered}
$$

where $H_{k}$ is the transformation between the state vector and measurement, in this case taken as $1, z_{k}$ is the current LIDAR measurement, and $R$ is the measurement covariance. Next, the Kalman gain $K_{k}$ is found by 


$$
K_{k}=\epsilon_{k} H_{k}^{\top}\left(H_{k} \epsilon_{k} H_{k}^{\top}+R\right)^{-1} .
$$

The Kalman gain is a weighting factor for the measurement signal; the closer the Kalman gain is to 1 , the more weight is applied to the measurement than the estimate, and vice versa. The third step is to solve fore the current state estimate $\hat{x}_{k}$ by

$$
\hat{x}_{k}=\hat{x}_{k}^{\prime}+K_{k}\left(z_{k}-H_{k} \hat{x}_{k}^{\prime}\right),
$$

where $\hat{x}_{k}^{\prime}$ is the system state of the prior time step. The covariance matrix is updated for the next time step by

$$
\epsilon_{k}=\left(I-K_{k} H\right) \epsilon_{k}^{\prime}\left(I-K_{k} H\right)^{\top}+K_{k} R K_{k}^{\top}+Q,
$$

where $I$ is an identity matrix equal to size $K_{k} \times H, \epsilon_{k}^{\prime}$ is the covariance matrix of the previous time step, and $Q$ is the process noise covariance. In the model, using a measurement noise covariance $R$ value of 0.1 and process noise covariance $Q$ value of 0.01 was found to give satisfactory filtering results.

The MA, which acts as a low pass filter, is given by

$$
\bar{x}_{k}=\frac{1}{n_{s}} \sum_{n=0}^{n_{s}-1} x[k-n]
$$

where $\bar{x}_{k}$ is the system output at time step $k, n_{s}$ is the number of samples being averaged, and $x[k-n]$ is the input signal vector over $n$ indices. The time delay $\Delta_{M A}$ from using the filter is given by

$$
\Delta_{M A}=\frac{n_{S}-1}{2} .
$$

For the MA, a 5 value average was observed to not introduce enough lag for the filtered signal to be dissonant from the real-time value. An EMA filter averages all the values 
of the past data and uses a weighting factor to determine how much influence newer data has over previous data. The weight factor $w_{k, \lambda}$ is given by

$$
w_{k, \lambda}=\lambda w_{k, \lambda}^{\prime}+1
$$

where $\lambda$ is the forgetting factor and $w_{k, \lambda}^{\prime}$ is the previous value of the weighting factor. The forgetting factor $\lambda$ ranges from 0 to 1 , with 1 giving the most weight to the past data. Using the weighting factor $w_{k, \lambda}$, the EMA filtered value $\bar{x}_{k, \lambda}$ is derived as

$$
\bar{x}_{k, \lambda}=\left(1-\frac{1}{w_{k, \lambda}}\right) \bar{x}_{k, \lambda}^{\prime}+\left(\frac{1}{w_{k, \lambda}}\right) x_{k},
$$

where $\bar{x}_{k, \lambda}^{\prime}$ is the value of the EMA of the previous time step and $x_{k}$ is the current signal value entering the filter. A forgetting factor of 0.9 was found to give the best performance for filtering the LIDARs with the least amount of phase lag.

The 12 filtering tests comprised of 3 subgroups, where each subgroup had a main filter on the LIDAR readings and a secondary filter on the estimated ship angles. The secondary filtering options were either no filter, meaning only the LIDAR signal was filtered, a KF, a MA, or an EMA. Figure 3.30 is a bar graph of the filtering combinations displayed in Table 3.9, where the shaded red bars are the roll values and the solid blue bars are the pitch values. Every filtering combination reduced the RMSE compared to the unfiltered signal. To further interpret the data the signal to noise ratio was found for each test case. Figure 3.31 outlines the SNR for the roll and pitch values, where the shaded red bars are roll and solid blue bars are pitch. Cross analysis of the results displayed in Figures 3.30 and 3.31 indicate that although the RMSE may be low for some filtering combinations, the signal may still have high levels of noise. It was found that using a KF as a primary filter and an EMA as a secondary filter had good reduction in RMSE with adequate SNR for roll and pitch. 
Table 3.9: LIDAR filtering combinations. Trial numbers correlate to $\mathrm{x}$-axis of Figures 3.30 . \& 3.31

\begin{tabular}{|c|c|c|c|c|c|c|c|c|c|c|c|c|c|}
\hline Trial & 1 & 2 & 3 & 4 & 5 & 6 & 7 & 8 & 9 & 10 & 11 & 12 & 13 \\
\hline $\begin{array}{c}\text { Primary } \\
\text { Filter }\end{array}$ & N.A & \multicolumn{9}{|c|}{ KF } & \multicolumn{5}{|c|}{ EMA } & \multicolumn{7}{c|}{ MA } \\
\hline $\begin{array}{c}\text { Secondary } \\
\text { Filter }\end{array}$ & N.A & N.A & KF & EMA & MA & N.A & KF & EMA & MA & N.A & KF & EMA & MA \\
\hline
\end{tabular}

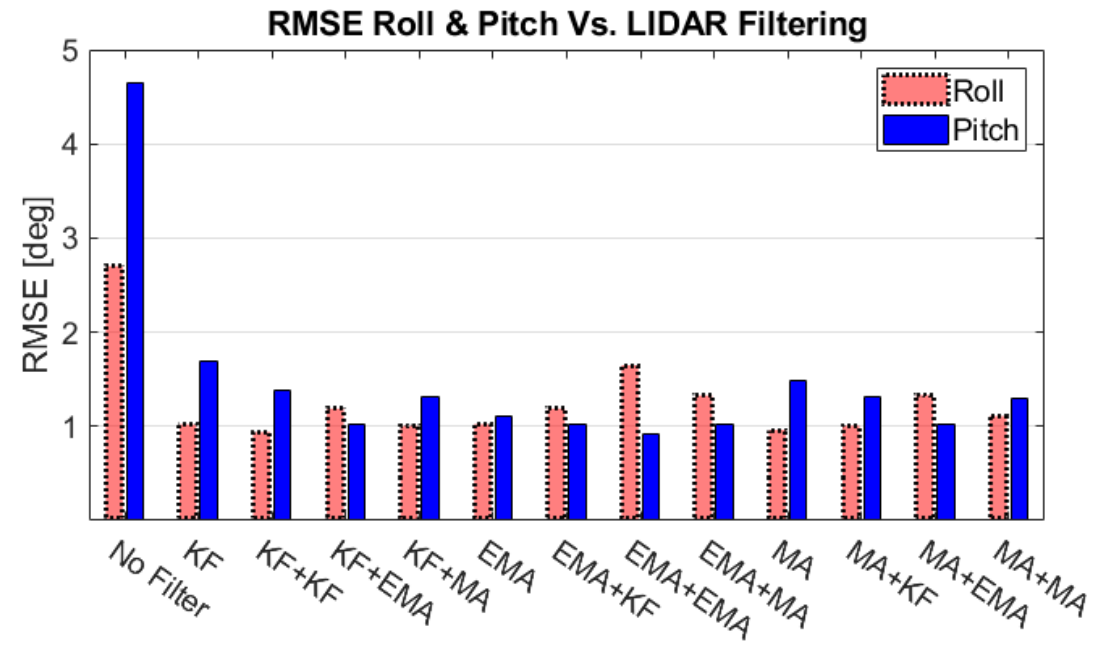

Filtering Method

Figure 3.30: LIDAR noise Filtering RMSE results for improving LIDAR based ship pose detection.

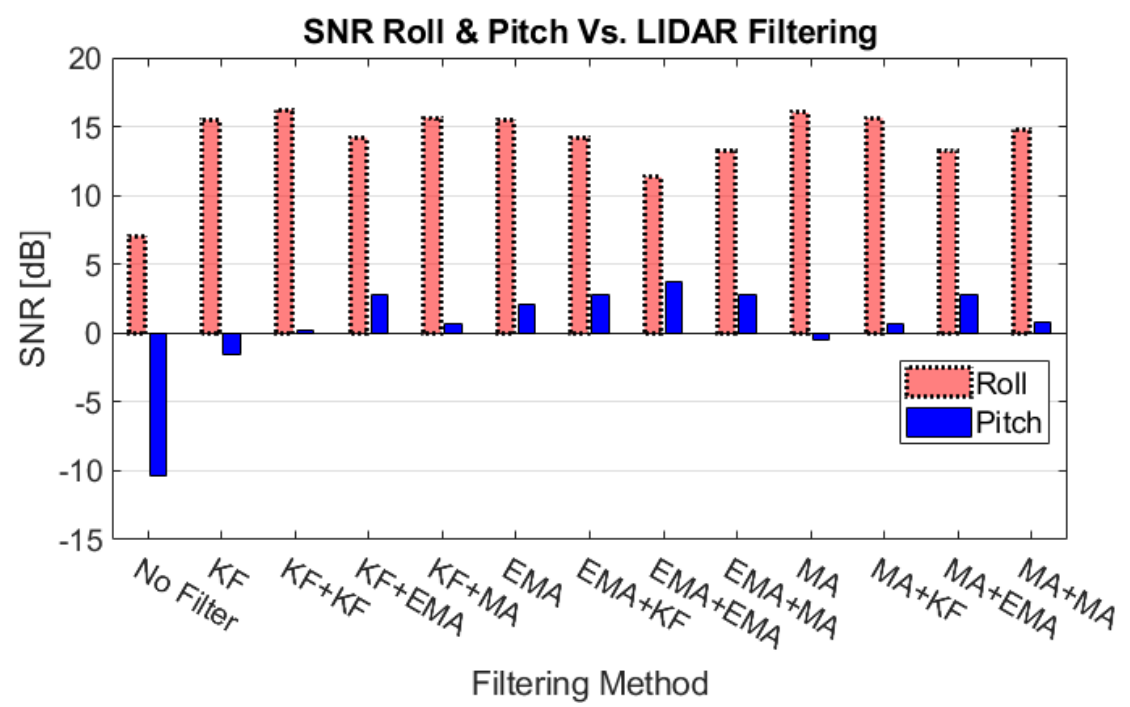

Figure 3.31: LIDAR noise Filtering SNR results for improving LIDAR based ship pose detection.

As previously stated, a KF is used as the primary filter and an EMA as the 
secondary. The combination of the KF and EMA was found to not introduce enough phase lag to degrade landing performance. Although the combination of the KF and EMA was found to be satisfactory for the simulation, the conditions in the synthetic environment remain under idealized circumstances as other sources of error were not included. Physical testing of the LIDARs should be performed to further validate this study. If sensor drift, propeller vibration, and ship deck texture are considered it is hypothesised that a more robust method of acquiring ship pose may be required. In future models and after physical testing, flash LIDARs may be introduced. A flash LIDAR incorporates a 2D array of photo diode sensors capable of detecting a point cloud at every time step. A large point cloud allows for a multitude of normal vectors to be found for the ship. Using neighbor polling, a technique which compares normal vectors in a point cloud to each other, it would be more likely normal that the true normal vector of the ship would be found. It was found that adding a fourth LIDAR and using the techniques outlined in Section 3.8.2 to estimate ship angles improved the RMSE by over $20 \%$ in some tests. Until ship estimation techniques are improved upon, the UAV uses an array of four LIDARs, with a KF on each LIDAR and a final EMA filter to smooth the roll and pitch angles determined by the pose detection algorithm. With the ship motion obtained, the UAV is now able to begin signal prediction for landing.

\subsection{Summary}

In this chapter the methodology used to develop a UAV capable of autonomous flight was described, contributing toward the first objective of this thesis. The fidelity of the synthetic environment was increased by including aerodynamic drag, atmospheric turbulence and wind gusts. The operational limits of the UAV were also found. A case study concluded that the UAV is capable of tracking a ship's landing zone movement 
in all sea states, however, the UAV is unable to do so in winds above $10 \mathrm{~m} / \mathrm{s}$. The effect of ship heading on ship motion was assessed and the opportune landing times for each sea state were highlighted. Finally, a LIDAR system was emulated and filtered for determining ship pose.

In the next chapter, the signal prediction algorithm used for determining landing Go states and active heave compensation is described, followed by the formulation of a self-tuning landing period indicator. 


\section{Chapter 4}

\section{Flight Control For Autonomous Landing at Sea}

In the previous chapter, the UAV was modeled and the synthetic environment was established. This chapter focuses on the flight control systems and simulated hardware used for landing the UAV on a ship. In Section 4.1, the signal prediction algorithm used for landing is examined and its operational parameters are highlighted. The development of an Active Heave Compensation (AHC) system that uses the Signal Prediction Algorithm (SPA) follows, and a case study reviewing its performance is presented. Section 4.3 details the formulation of a Landing Period Indicator (LPI) and how it may be trained on-line to forecast safe landing times without the use of signal prediction. A case study outlining the LPI's performance in comparison to the SPA is included. Finally, Section 4.4 closes the chapter with the introduction of a state-machine flight controller and how it is used to autonomously land the UAV using the various landing methodologies discussed in this chapter. 


\subsection{Signal Prediction Algorithm}

A signal prediction algorithm developed by Kuchler et al [84], used by Woodacre et al. [7], and further developed by McPhee and Irani [8] is used extensively throughout the presented work to predict ship motion. Ship motion, which is primarily influenced by wave actions, may be decomposed into a set of $N$ sine waves using an FFT such that the ship motion $s$ can be described as function of time $t$

$$
s(t)=\sum_{i=1}^{N} A_{i} \sin \left(2 \pi f_{i} t+\phi_{i}\right)+v(t),
$$

where $N$ is the number of modes observed, $A_{i}, f_{i}$, and $\phi$ are the amplitude, frequency, and phase of the $i^{\text {th }}$ mode, respectively, and $v$ is the offset for non zero mean bias. The FFT requires an initialization period $t_{F F T}$ where the ship motion is concatenated over the length of $t_{F F T}$ before the sequence can be decomposed into $N$ modes. The resolution $R_{F F T}$ of the FFT is given by

$$
R_{F F T}=\frac{f_{s}}{n_{s}}=\frac{1}{t_{F F T}},
$$

where $f_{s}$ is the sampling frequency of the ship motion, and $n_{s}$ is the number of samples in the bin of length equal to $t_{F F T}$. By the Nyquist Frequency Theorem the maximum observable frequency is half of the the sample rate $f_{s}$, for ship motion a high resolution is desired. Therefore, the FFT window $t_{F F T}$ should be selected to reflect the highest resolution necessary to obtain the modes required to predict the ship motion over the prediction horizon $T_{\text {Pred }}$. In order to use an FFT for on-line predictions an observer model is initialized. 


\subsubsection{SPA Derivation}

At every FFT interval, a peak detection algorithm is used to select the dominant modes and their corresponding amplitudes $A_{F F T}$ and frequencies $f_{F F T}$. The parameters of the modes identified by the FFT are then used to initialize a discrete observer model given by

$$
\hat{x}_{k+1}=\Lambda \hat{x}_{k}+L\left(s_{k}-\hat{s}_{k}\right), \quad \hat{s}_{k}=C \hat{x}_{k},
$$

where $\hat{x}$ is the vector of observed states, $k$ is the current time step, $\Lambda$ is the discrete system matrix, $L$ is a matrix of observer gains, $s$ is the measured signal, $\hat{s}$ is the estimated signal, and $C$ is the system output matrix. The system output matrix, discrete system matrix, observed states, and gain matrix, were implemented in the model in accordance to [84] and in collaboration with McPhee [8,9]. Summing over all modes and accounting for static offset $v_{t}$ the observer model $\dot{x}$ is

$$
\dot{x}=\left[\begin{array}{ccccc}
B_{1} & 0 & \cdots & \cdots & 0 \\
0 & B_{2} & \ddots & \cdots & \vdots \\
\vdots & \ddots & \ddots & \ddots & \vdots \\
\vdots & \ddots & \ddots & B_{N} & 0 \\
0 & \vdots & \vdots & 0 & 0
\end{array}\right]\left[\begin{array}{c}
x_{1} \\
x_{2} \\
\vdots \\
x_{N} \\
x_{v}
\end{array}\right] \quad s(t)=\left[\begin{array}{lllll}
C_{1} & C_{2} \cdots & C_{N} & 1
\end{array}\right],
$$

where $B_{i}$ is solved for every mode as

$$
B_{i}=\left[\begin{array}{cc}
0 & 1 \\
-\left(2 \pi f_{i}\left(t_{0}\right)\right)^{2} & 0
\end{array}\right]
$$

Equation 4.4 is then discretized for on-line implementation giving 


$$
x_{k+1}=\exp (B \Delta T) x_{k}, \quad s_{k}=C x_{k},
$$

where $\Delta T$ is the system sampling time and $B \Delta T$ represents the discrete system matrix $\Lambda$. The states for a single mode, estimated by the observer, may be written as

$$
\begin{gathered}
\hat{x}_{i, 1}=A_{O b s, i, k} \sin \left(2 \pi f_{i} t_{k}+\Phi_{o b s, i, k}\right), \\
\hat{x}_{i, 2}=2 \pi A_{O b s, i, k} f_{i} \cos \left(2 \pi f_{i} t_{k}+\Phi_{o b s, i, k}\right) .
\end{gathered}
$$

$\hat{x}_{i, 1}$ and $\hat{x}_{i, 2}$ may be re-arranged to solve for the adapted phase $\Phi_{O b s, i, k}$ and amplitude $A_{o b s, i, k}$ of the observer

$$
\begin{gathered}
\Phi_{O b s, i, k}=\arctan \left(\frac{2 \pi f_{i} \hat{x}_{i, 1}}{\hat{x}_{i, 2}}\right)-2 \pi f_{i} t_{k}, \\
A_{O b s, i, k}=\frac{\hat{x}_{i, 1}}{\sin \left(2 \pi f_{i} t_{k}+\Phi_{O b s, i, k}\right.} .
\end{gathered}
$$

The frequency content of the modes are not estimated using an observer due to the high accuracy of the FFT. Substituting the adapted parameters into Equation 4.1, the signal forecast over the prediction horizon is

$$
S_{\text {Pred }}\left(t_{0}+T_{\text {Pred }}\right)=\sum_{i=1}^{N} A_{\text {Obs }, i, k} \sin \left(2 \pi f_{i} t+\Phi_{\text {Obs }, i, k}\right)+v(t) .
$$

Equation 4.11 is what is used in the simulator for predicting ship motion. In summary, an FFT and peak detection algorithm are used to evaluate ship motion over a specified time interval corresponding to the desired frequency resolution. The modes detected by the FFT are used to initialize an observer which adapts the mode 
parameters online based on the disagreement between the predicted signal and the measured signal. The observer is required for on-line prediction as the mode parameters found by the FFT are mean values over the entire FFT window and would not result in an accurate prediction. The adapted observer parameters are recomposed into sine waves and summed over the prediction horizon $T_{\text {pred }}$, resulting in the predicted signal. The scope of this thesis relates to the implementation of the SPA, and more in depth analysis on the derivation of the SPA is provided in $[7,8,84]$.

To facilitate a safer landing, the SPA is used to predict ship motion over the prediction horizon and evaluate if the ship motion is within the Ship Helicopter Operating Limits (SHOL). To predict ship roll and pitch, independent SPA's must be used for each signal. Figure 4.1 provides the information structure of the SPA, where the inputs into the system are: FFT window $\left(t_{F F T}\right)$, ship motion, prediction horizon $\left(T_{P r e d}\right)$, and the Go Threshold. Two SPAs are used in parallel to predict the ship's roll motion and pitch, if both magnitudes of the predicted signals are less than their threshold values ( $5^{\circ}$ roll, $2^{\circ}$ pitch) a Go signal enables the UAV state transition from low hover to land.

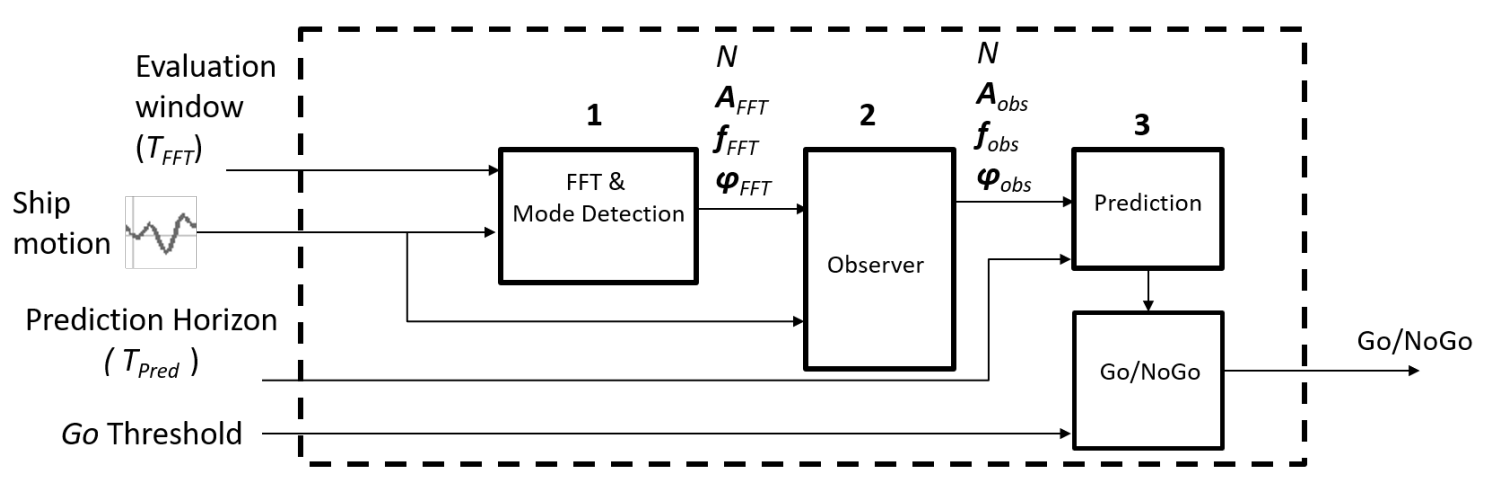

Figure 4.1: Information structure of the Signal Prediction Algorithm [9] 


\subsubsection{SPA Prediction Horizon for Landing}

During landing operations, the UAV transitions from its low hover state to a landing state if the predicted roll and pitch values are less than their thresholds. During landing the prediction horizon input to the SPA decreases as a function of the estimated remaining time to land. In this thesis, the UAV transition time from a low hover to a grounded state is allotted $5 \mathrm{~s}$. However, due to heave motion of the ship, the time required to land may be less or exceed $5 \mathrm{~s}$. Therefore, the prediction horizon of the SPA is constantly adjusted based off the estimated time to land. Figure 4.2, displays a flow diagram of the UAV landing procedure when using the SPA. Once above the Landing Zone (LZ) the SPA collects data to initialize. Once initialized, the UAV proceeds to its low hover position. The SPA's prediction horizon is adjusted based on the UAV's low hover position and landing velocity. If the roll and pitch SPA predict it is safe to land, the SPA outputs a Go command, and the UAV lowers its altitude while also shortening the prediction horizon. While landing, if a SPA predicts the ship motion will exceed the threshold values, the SPA outputs NoGo and the UAV aborts landing to return to its low hover position. However, if the UAV is within $0.5 \mathrm{~m}$ to the deck it will land, based on the assumption that it is safer to land than abort while in close proximity to the ship.

Depending on the user selected simulation settings, the flight controller will enter into one of three landing modes. Each landing mode affects how the prediction horizon is calculated, however, each landing mode attempts to land the UAV within $5 \mathrm{~s}$. The low hover position of the UAV is either $2.5 \mathrm{~m}$ or $5 \mathrm{~m}$, where a $5 \mathrm{~m}$ low hover with a $5 \mathrm{~s}$ landing time is common for rotor crafts [85], and $2.5 \mathrm{~m}$ would be considered lower

than average. Landing Mode $1\left(M_{1}\right)$ corresponds to a low hover position of $2.5 \mathrm{~m}$ above the ship deck and a ramp landing velocity $V_{L 1}$ of $0.5 \mathrm{~m} / \mathrm{s}$, giving the prediction horizon 


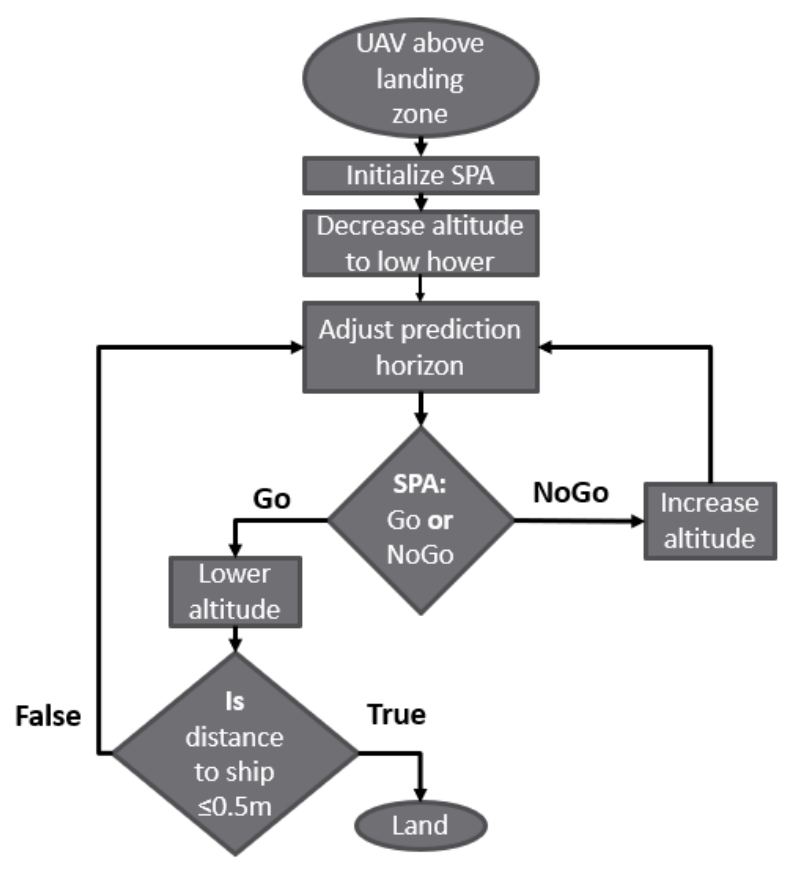

Figure 4.2: UAV landing protocol using the SPA.

$$
T_{\text {Pred }}\left(M_{1}\right)=\frac{L_{d}}{V_{L 1}}
$$

where $L_{d}$ is the distance to the ship deck measured by the UAV's LIDARs. Landing Mode $2\left(M_{2}\right)$ has a low hover altitude of $5 \mathrm{~m}$ and follows a piece-wise function to determine its linear-ramp landing velocity given by

$$
V_{L 2}\left(M_{2}\right)=\left\{\begin{array}{ll}
-2 \frac{m}{s}, & L_{d}>3 m \\
-1 \frac{m}{s}, & 3 m \leq L_{d}>1 m \\
-0.5 \frac{m}{s}, & 1 m \leq L_{d}
\end{array} .\right.
$$

The landing velocities from Equation 4.13 are used to calculate how long the prediction horizon has to be based on the UAV's low hover position by

$$
T_{\text {Pred }}\left(M_{2}\right)=\left\{\begin{array}{ll}
\frac{L_{d}-3}{\left|V L_{2}\right|}+4, & L_{d}>3 m \\
\frac{L_{d}-1}{\left|V L_{2}\right|}+2, & 3 m \leq L_{d}>1 m \\
\frac{L_{d}}{\left|V L_{2}\right|} & , \quad 1 m \leq L_{d}
\end{array},\right.
$$


where the constants 4 and 2 are the remaining time it will take the UAV to cycle through the next piece-wise function(s) until the UAV is landed. Landing Mode 3 $\left(M_{3}\right)$ has a low hover position of either $2.5 \mathrm{~m}$ or $5 \mathrm{~m}$ and has a dynamic landing velocity that is determined by the AHC system. The AHC system attempts to land the UAV in $5 \mathrm{~s}$ or less regardless of its position, thus the prediction horizon is

$$
T_{\text {Pred }}\left(M_{3}\right)=5-\Delta t_{\text {land }}
$$

where $\Delta t_{\text {land }}$ is the elapsed time since the Go signal was received.

\subsubsection{Go-NoGo Command Latching}

To remove unwanted fluctuation in the Go-NoGo command signal, McPhee and Irani [8] proposed a latching algorithm that latches to a Go or NoGo command. The proposed latching algorithm evaluates the incoming Go-NoGo commands, determined by the SPA, and latches to a command if no change is observed in the Go-NoGo command over an evaluation interval. Before re-evaluating and re-latching, a latched state will run for the selected time period, regardless of the incoming Go-NoGo states. For the purposes of this thesis, an evaluation interval of $0.25 \mathrm{~s}$ and latching period of $0.5 \mathrm{~s}$ were found to remove chatter and false Go signals in the SPA. In practice, physical tests should be used to evaluate both intervals which may change based on UAV response times and rates-of-descent.

\subsection{Active Heave Compensation using Signal Pre- diction}

While landing and station keeping at a low hover the risk of ship-UAV collision increases. Using the SPA to predict the future heave motion of the ship and com- 
pensate by systematically altering the UAV's altitude with respect to the deck lowers the chances of collisions. An estimation of the future position of the deck may also allow for lower low hover positions of the craft, even for crafts with slower dynamics. Further, the same AHC may be used to plan landing trajectories for the UAV when a Go signal to land is received.

\subsubsection{Active Heave Compensation - Low Hover}

The AHC algorithm utilizes a similar SPA to that used for roll and pitch predictions, however, instead of a Go or NoGo signal, the heave SPA returns an array of 51 points of the predicted heave positions for the next $5 \mathrm{~s}$. Operating at a $100 \mathrm{~Hz}$, a new prediction array is calculated every 0.01 seconds. An operating rate of $100 \mathrm{~Hz}$ was selected based off implementation of the SPA in $[7,8]$. To increase the resolution of the prediction, a piece-wise spline is fit to the data to increase the array size to 501 points. To reduce the risk of ship-UAV collision, the AHC system evaluates the predicted spline data for incoming high heave motion, where if high, the AHC system will command the UAV to preemptively raise its altitude. The time into the future $t_{A H C}$ that the AHC system will search for incoming heave motion and compensate the UAVs position should be tuned according to a UAVs dynamics. In this thesis, the UAV had a maximum ascent rate of $0.5 \mathrm{~m} / \mathrm{s}$ while in a low hover state and using $t_{A H C}=0.5 \mathrm{~s}$ was found to be effective in reducing UAV-ship collisions while at a $2.5 \mathrm{~m}$ low hover. The AHC was found not to be needed for a low hover position of $5 \mathrm{~m}$.

\subsubsection{Active Heave Compensation - Landing}

Aside from actively searching for future positions of the ship deck and adjusting the UAV's low hover position, the AHC algorithm continually updates landing trajectories. The goal while trajectory planning is to search for a landing velocity which will 
result in a desired impact velocity between the UAV and ship. As the UAV attempts to maintain a fixed distance between the UAV and the ship when in a low hover, the required landing distance remains fairly constant. However, once the UAV begins to land the distance between the ship and UAV will change based on ship motion and UAV landing velocity. The trajectory planner operates as follows:

- The AHC searches for UAV landing trajectories by taking the difference between the UAV's current position and the position of the ship deck for the next 5 seconds. Figure 4.3 exemplifies landing trajectories found by the AHC by showing the UAV's current position as a red dot, and 5 different landing paths, dotted magenta lines, the UAV could take to the predicted position of the deck. The predicted heave motion is the red dotted-dashed line coming off the solid blue line which is the past heave motion. The black crosses at the end of the landing trajectories are the estimated points of touch down for the UAV. Each landing path requires a unique landing velocity to reach the ship deck at the specified time. In Figure 4.3 only 5 landing trajectories were displayed, however, a landing trajectory is planned to reach every point in the predicted heave position array.

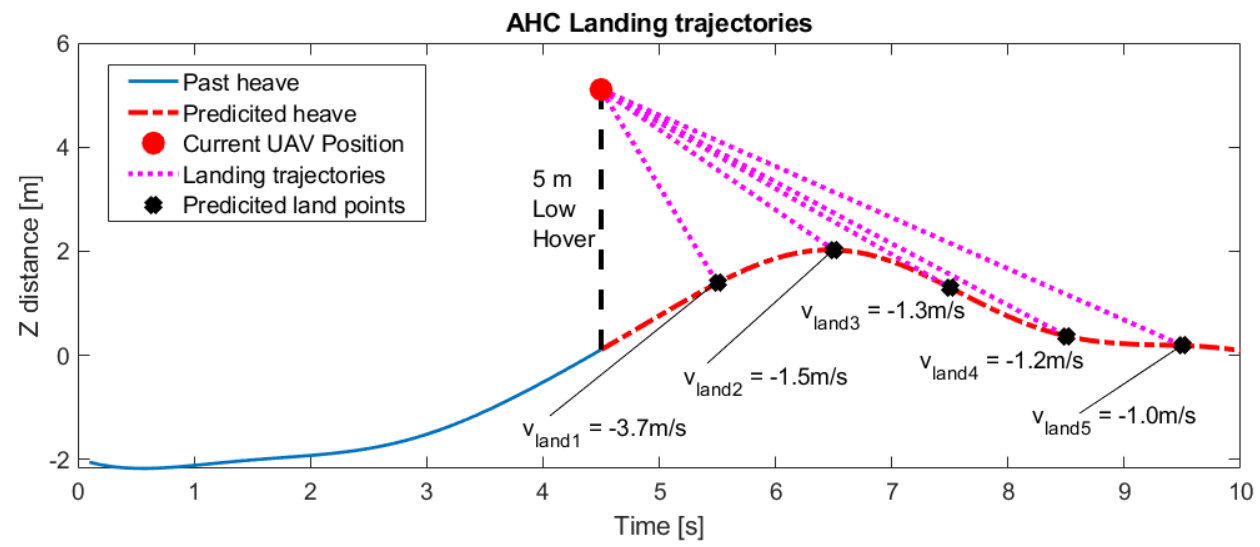

Figure 4.3: Using the signal prediction algorithm to develop landing trajectories for the UAV.

- The predicted impact velocity between the UAV and the ship is found for each 
of the landing velocities by taking the difference between the landing velocities and the derivative of the ship's positions at the corresponding index. Figure 4.4 displays the corresponding absolute impact velocity values between the UAV and ship for $t=5.5 \mathrm{~s}$ to $9.5 \mathrm{~s}$. The target impact velocity $0.5 \mathrm{~m} / \mathrm{s}$ and the greatest allowable impact velocity $1 \mathrm{~m} / \mathrm{s}$ are indicated by the dashed black and red lines respectively. For this set of data there are two times, $t=7.4 \mathrm{~s}$ and $t=9.4 \mathrm{~s}$, where the impact velocity meets the goal value, and a total of $2.5 \mathrm{~s}$ that are less than threshold limit of $1 \mathrm{~m} / \mathrm{s}$.

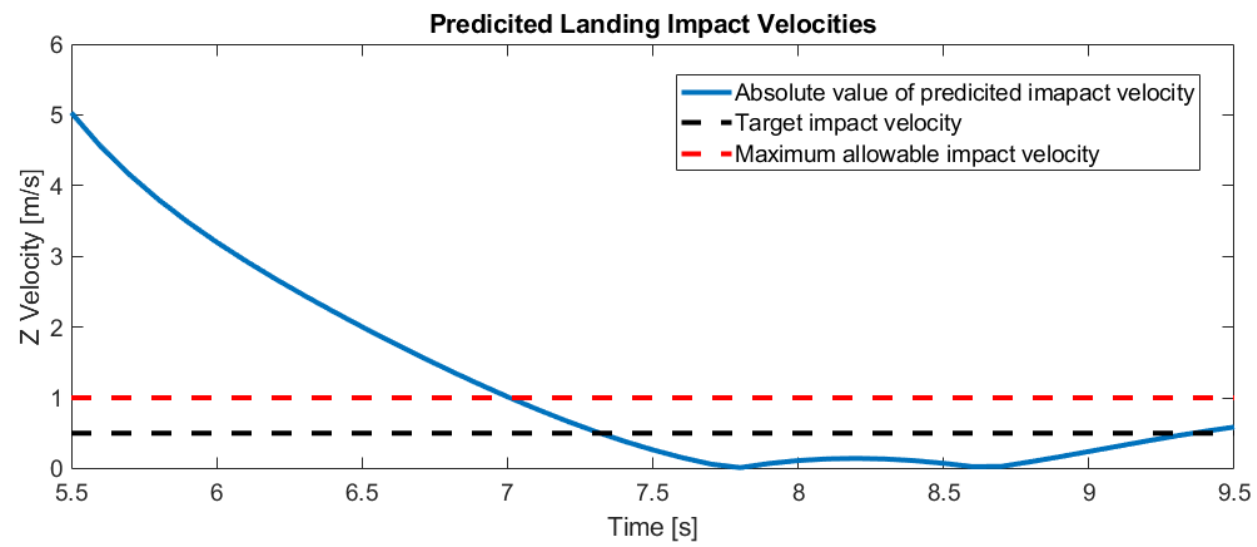

Figure 4.4: Predicted impact velocities from the AHC landing trajectories.

- A gradient search is performed on the array of 501 impact velocities to search for a landing velocity which most closely meets the impact velocity goal of $0.5 \mathrm{~m} / \mathrm{s}$. If that impact velocity is unobtainable, a search is performed for a impact velocity of $0.5 \pm 0.1 \mathrm{~m} / \mathrm{s}$ and so on until a match is found. If multiple landing trajectories meet the goal value, the trajectory closest in time is selected. However, landing trajectories will only be considered if the required landing velocity is less than $1.5 \mathrm{~m} / \mathrm{s}$.

- If a Go command is received for landing, the UAV will follow the planned landing trajectory. During descent, the UAV continues to search for landing trajectories that are closest to the impact velocity goal, and if one is found the 
UAV will alter its descent path.

- At anytime during landing, if a NoGo signal is received the UAV will return to its low hover position unless the relative distance between the vessel and the craft is less than $0.5 \mathrm{~m}$.

The SPA requires a zero mean signal to have an accurate prediction, or if biased, the offset must be known. The need for a zero mean signal complicates the acquisition of heave motion at the LZ as heave is not oscillating about a fixed axis. Further, heave motion is coupled with ship pitch and as a result of the LZ's location at the stern of the ship, the heave motion observed at the LZ is amplified. As the UAV has to acquire the heave motion absent deck infrastructure, one solution is to define ship movement in the global frame. Obtaining the heave motion in the global frame is accomplished by subtracting the UAV's altitude from the LIDAR's measurements as depicted in Figure 4.5. Working in the global frame however does not address the issue of finding the heave oscillatory axis zero, due to the water line not matching the global sea level. Instead, the static water line correlates to sea swell and wave actions. A moving average filter with a $10 \mathrm{~s}$ window was used to estimate the bias between the sea line and the global frame zero. The bias is then subtracted from the heave motion resulting in zero mean heave motion which may be used by the SPA for heave compensation. With the AHC system developed, its performance can be evaluated. 


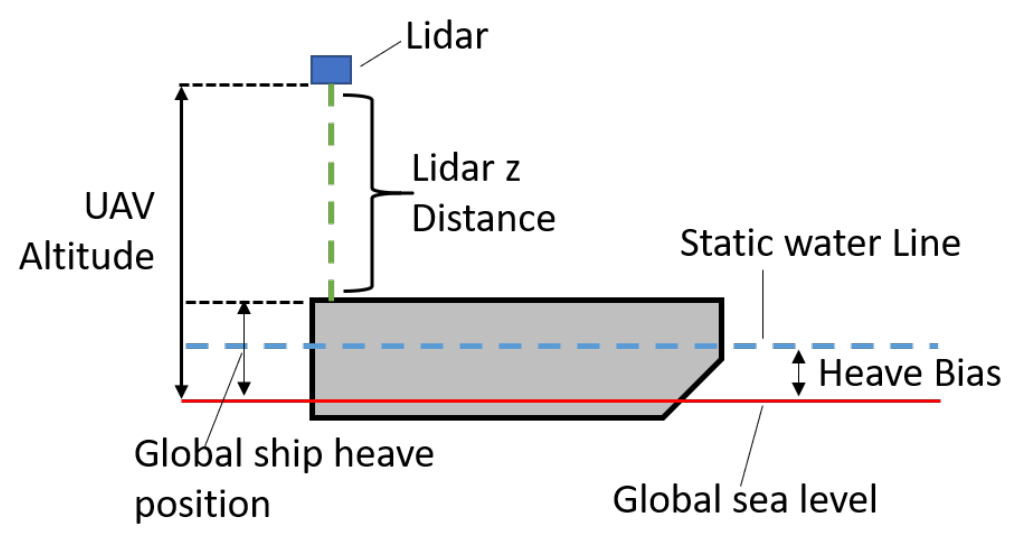

Figure 4.5: Determining ship heave motion in the global coordinate frame.

\subsubsection{Performance of AHC for landing}

The performance of the AHC system for landing greatly depends on the reliability of the predictions, where a poor prediction may result in a higher impact velocity than if it were to land using one of the ramp landing velocity methods described in Equations 4.12 and 4.13. To evaluate the performance of the AHC to the standard ramp landing velocity methods, four test groups were assembled. Two of the test groups landed using the AHC algorithm and two landed using the standard ramp methods. Low hover positions of $2.5 \mathrm{~m}$ and $5 \mathrm{~m}$ were used for each landing method. The tests comprised of ship motion in sea states 4,5 and 6 with ship heading's of $30^{\circ}$, $60^{\circ}$, and $90^{\circ}$. To ensure a controlled testing environment, atmospheric turbulence and wind gusts were disabled in the model and each UAV was commanded to land at $200 \mathrm{~s}$ into the simulation. Table 4.1 summarizes the results obtained from the 48 tests. The total number of simulations which had an impact velocity greater than $1 \mathrm{~m} / \mathrm{s}$ is reported, along with the average landing velocity and the standard deviation. Both the $2.5 \mathrm{~m}$ and $5 \mathrm{~m}$ low hover test cases using the AHC had 3 UAVs land with an impact velocity greater than $1 \mathrm{~m} / \mathrm{s}$. Without the AHC system, the UAV with a $2.5 \mathrm{~m}$ low hover had 6 UAVs land with an impact velocity greater than $1 \mathrm{~m} / \mathrm{s}$, and the $5 \mathrm{~m}$ low hover had 7 . Using the AHC, the average landing velocities for 
both low hover cases were below $1 \mathrm{~m} / \mathrm{s}$, however, the average did not meet the goal impact velocity of $0.5 \mathrm{~m} / \mathrm{s}$. The UAVs which landed based on the ramp functions each had higher impact velocities than their AHC counter parts, and the average landing velocity for the $5 \mathrm{~m}$ low hover case exceeds the maximum allowable value of $1 \mathrm{~m} / \mathrm{s}$. Further, the standard deviation values are lower with the AHC system indicating that the AHC system is more consistent in landing the UAV with an impact velocity closer to the goal value. The results indicate that using signal prediction for determining landing trajectories can improve landing performance, the next section seeks to improve landing performance without the use of signal prediction.

Table 4.1: Impact velocity comparison between using the standard landing methods and using the active heave compensation algorithm for headings of $0^{\circ}, 30^{\circ}, 60^{\circ}$, and $90^{\circ}$ in sea states 4,5 , and 6 .

\begin{tabular}{|c|c|c|c|c|}
\hline $\begin{array}{c}\text { Landing } \\
\text { Mode }\end{array}$ & $\begin{array}{c}\text { Low Hover } \\
\text { Position } \\
{[\mathrm{m}]}\end{array}$ & $\begin{array}{c}\text { \# of Trials with } \\
\text { Landing Velocity } \\
>1 \mathrm{~m} / \mathrm{s} \text { (out of } 12)\end{array}$ & $\begin{array}{c}\text { Avg. Landing } \\
\text { Velocity }[\mathrm{m} / \mathrm{s}]\end{array}$ & $\begin{array}{c}\text { Std. Landing } \\
\text { Velocity }[\mathrm{m} / \mathrm{s}]\end{array}$ \\
\hline No AHC & 2.5 & 6 & 0.94 & 0.51 \\
\hline AHC & 2.5 & 3 & 0.68 & 0.38 \\
\hline No AHC & 5 & 7 & 1.16 & 0.52 \\
\hline AHC & 5 & 3 & 0.80 & 0.41 \\
\hline
\end{tabular}

\subsection{Landing Period Indicator}

Alternative methods of indicating landing conditions were investigated during this research. Ferrier et al. [86] used the energy index (EI) to predict quiescent periods in the ship motion using

$$
E I=a_{1} \dot{w}^{2}+a_{2} \ddot{w}^{2}+a_{3} \dot{j}^{2}+a_{4} \ddot{j}^{2}+a_{5} \dot{p}^{2}+a_{6} \ddot{p}^{2}+a_{7} \dot{q}^{2}+a_{8} \ddot{q}^{2},
$$


where $a_{1-8}$ are weighted dynamic coefficients, $w$ is heave, $j$ is sway, $p$ is roll, and $q$ is pitch. Ferrier et al. [86] state that the dynamic coefficients are tuned on-line based off the "aircraft limitation scale" and ship motion parameters. Ferrier et al. [86] claim good agreement between their predicted energy and ship motion, however it is unclear to this author how they established their dynamic coefficients. Furthermore, EI only considers the cumulative motion of the ship and does not consider if the ship is within safe thresholds. Based off Ferrier et al.'s principle of EI, the author of this thesis developed a Landing Period Indicator (LPI) independent of vessel and aircraft type without the need for ship-UAV communication or signal prediction. The developed LPI system is presented using ship motion from sea state 3 . The initiative of the LPI was to develop a system which can analyze past ship data, find correlations in the motion of when it was safe to land and use that information to have a higher chance of a successful landing without the use of signal prediction. Furthermore, the LPI was developed to be computationally inexpensive while having comparable or improved results over the SPA.

\subsubsection{Proposed Landing Period Indicator}

Ship motion in sea state 3 was analyzed to assess whether correlations exist between the ship's dynamic states and periods of sustained quiescence where the ship motion was within the roll and pitch thresholds, $5^{\circ}$ and $2^{\circ}$ respectively. An algorithm was developed to observe ship motion over a specified time and collect data from when the ship's motion was within the Go threshold values for $5 \mathrm{~s}$ or more, however, if no data is found, the LPI searches for the next grouping of Go times closest to 5 s. Figure 4.6 displays the values obtained during the initialization phase of the algorithm, where the blue line, when high, indicates that the ship motion is less than the limit and when low exceeds the limit. The red box indicates periods where the ship motion was within the threshold limits for an excess of 5 seconds. For this set of data, there were 
5 instances which met the criteria for landing.

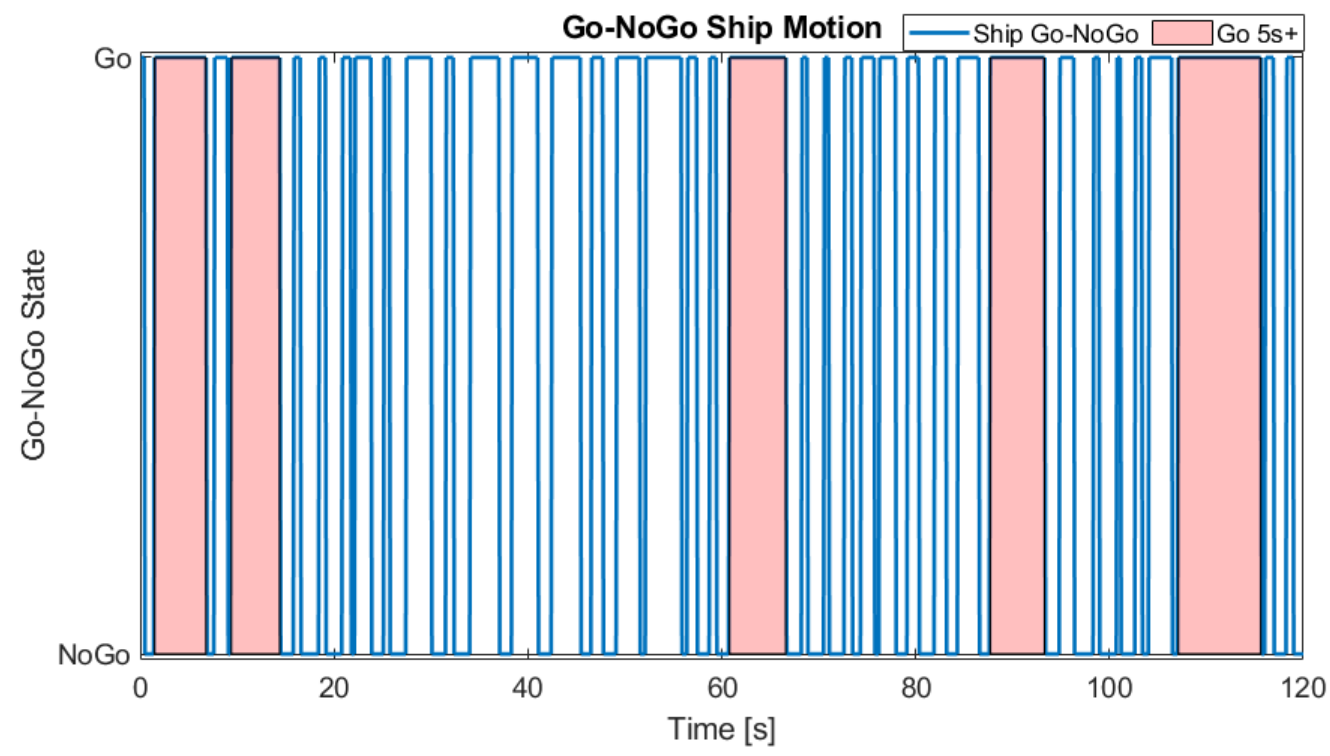

Figure 4.6: 120 second evaluation of ship motion for Go periods lasting longer than 5 seconds.

Next, the dynamic ship motion behaviours were observed during the instances where it would have been safe to land. Ship roll velocity, pitch velocity, and heave velocity were the three dynamic properties under evaluation. Ideally the energy $K_{e}$ of the ship would be evaluated for each signal using

$$
K_{e}=\frac{1}{2} m v_{i}^{2},
$$

where $m$ is the mass of the ship, and $v_{i}$ is the velocity component of either roll, pitch or heave. It is assumed that the mass of the ship is unknown, therefore as a metric for ship energy, the Energy Index (EI) will be taken as the square of the velocity terms $v_{i}^{2}$. Figure 4.7 displays the EI of the ship over the evaluation period of $120 \mathrm{~s}$, where the first subplot is the square of the ship's roll velocity, the second subplot is the square of the ships pitch velocity and the third subplot is the square of the heave velocity. The red boxes in Figure 4.7 indicate the Go periods sustained for $5 \mathrm{~s}$ or longer and are the values from Figure 4.6. The units for heave energy $\left(\mathrm{m}^{2} / \mathrm{s}^{2}\right)$ are 

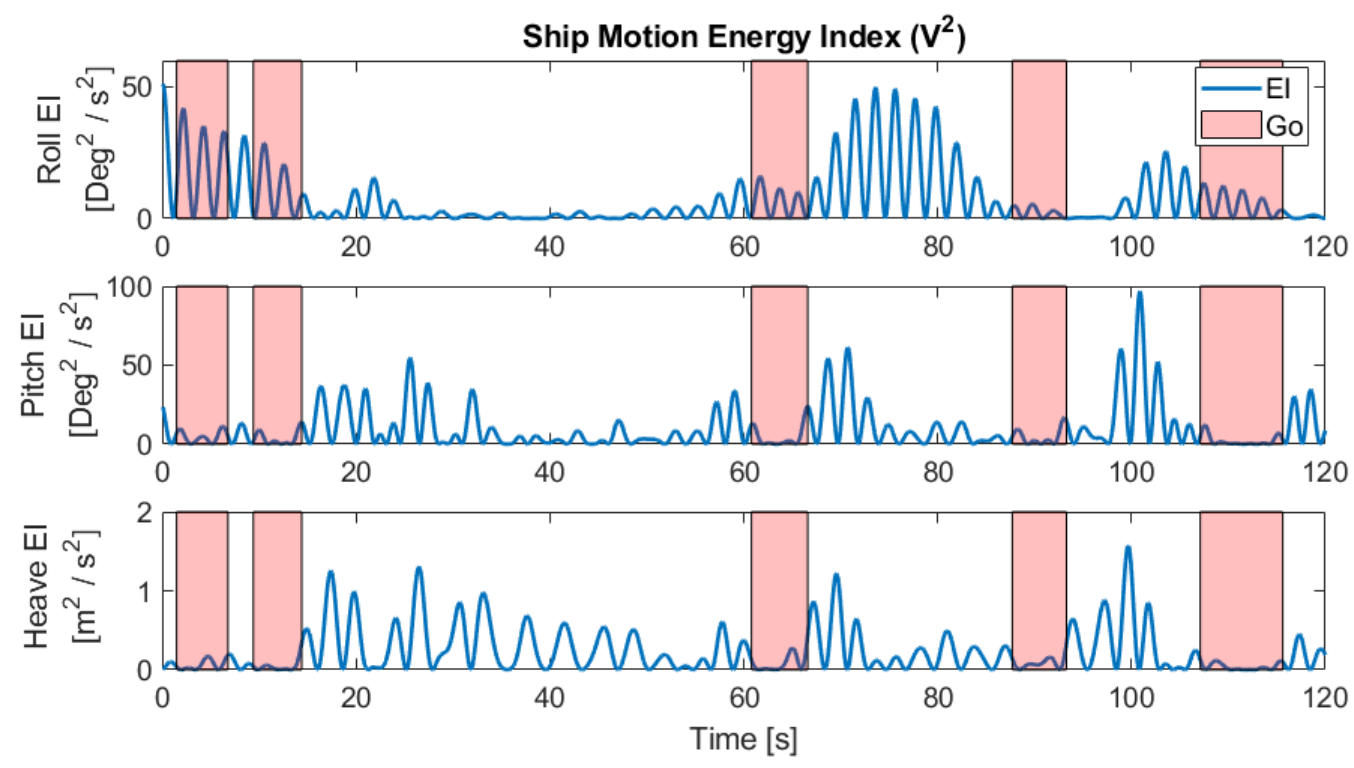

Figure 4.7: Energy index of ship roll pitch and heave.

incompatible with roll and pitch units $\left(\operatorname{deg}^{2} / \mathrm{s}^{2}\right)$. To rectify the unit difference, each set of energy data was normalized using the average maximum value observed during the sustained Go periods. The normalization of Figure 4.7 is displayed in Figure 4.8, where the red box amplitudes are at a value of 1 for each sustained Go period.

To further disparage data which may be an anomaly or insignificant to the set, the calculated normalization coefficients were only computed from the Go period values which were within half of a standard deviation from the cumulative average. The normalization routine is used for finding the normalizing coefficient's for roll, pitch and heave denoted as $N_{p}, N_{q}, N_{w}$ respectively. The total indicated EI of the ship is represented as the average value of the sum of the ship's normalized EIs, where a value greater than 1 indicates high ship energy not suitable for landing. The total value of $E I$ is written as

$$
E I=\frac{1}{3}\left(N_{p} \dot{p}^{2}+N_{q} \dot{q}^{2}+N_{w} \dot{w}^{2}\right)
$$

where $\dot{p}, \dot{q}, \dot{w}$ are the ship's roll, pitch, and heave velocities. The normalized rate of 

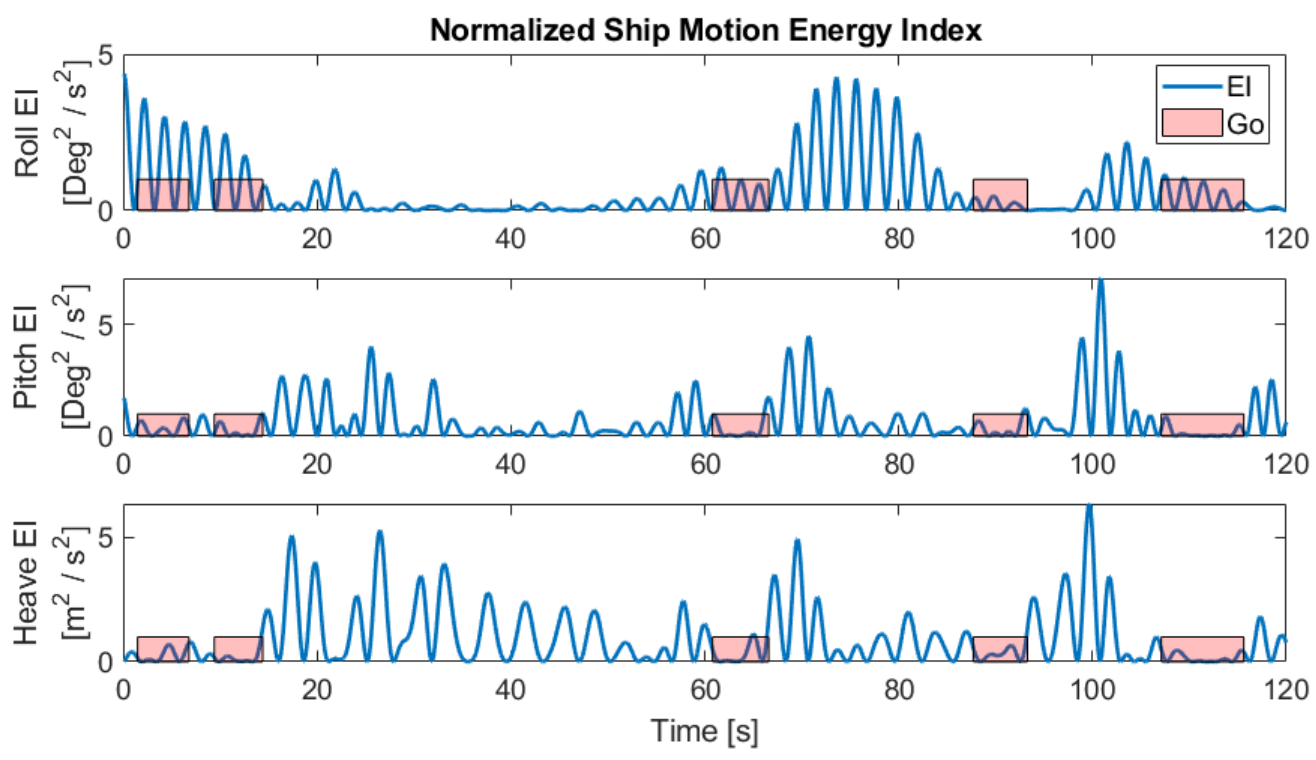

Figure 4.8: Normalized energy index of ship roll pitch and heave.

change of the energy index $\dot{E} I$ is taken as the absolute value of the derivative of the calculated EI, written as

$$
\dot{E I}=N_{D E I}\left|\frac{\partial E I}{\partial t}\right|,
$$

where $N_{D E I}$ is the normalizing coefficient for $\dot{E I}$, and is found using the same routine used to calculate $N_{p}, N_{q}$, and $N_{w}$. The calculated energy index $E I$ and its rate of change $\dot{E} I$ are weighted and amalgamated to form the landing period indicator given as

$$
L P I=G_{1} E I+G_{2} \dot{E} I
$$

where weighting gains $G_{1}$ and $G_{2}$ are used to alter the influence of each parameters. For example, by weighting $G_{2}$ heavier than $G_{1}$, the value of $L P I$ would be more sensitive to the rate of change of energy rather than the current energy. The weighting factors may be tuned to desired performance, yet noting that they must always sum to 1. It was found that equal weighting had satisfactory results from the cases studied. 


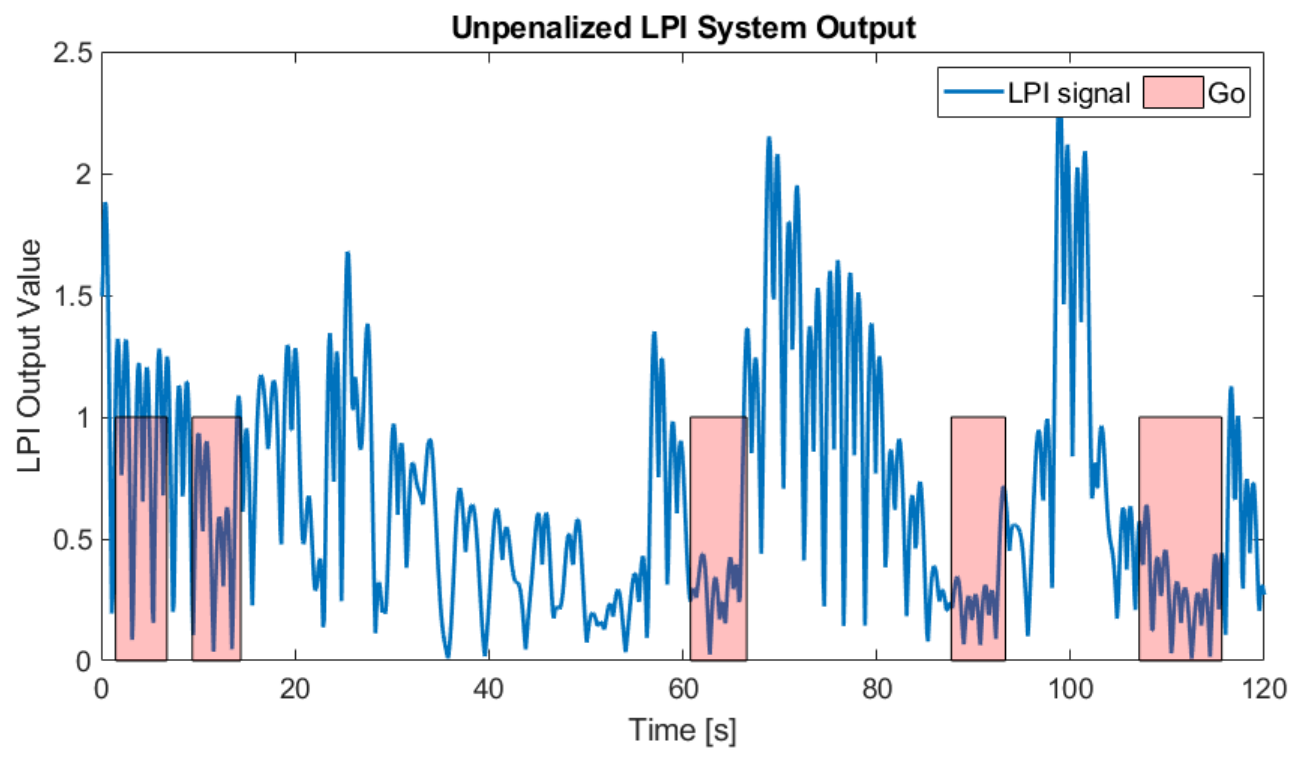

Figure 4.9: Un-penalized landing period indicator.

Figure 4.9 displays the LPI over the $120 \mathrm{~s}$ observation period, where values under 1 are considered opportune for landing. There are times when the values of the LPI are less than 1 even though it is not a favorable time to land. One scenario that leads to false LPI outputs is low frequency ship motion where the ship motion exceeds the threshold limits, but the system depicts the energy as low, to combat this during real time execution, the LPI will only output a value less than 1, meaning it's safe to land, if the current ship motion is within the threshold limits.

A ship motion penalty function $P_{L P I}$ was introduced to the system to improve the LPI performance. The penalty function serves to amplify the value of the LPI output when ship motion values are high. The penalty function is formulated to normalize the roll and pitch values about their respective threshold limits, take the average value of the two, scale that value by a gain and multiply the final penalty value by the LPI's original output. An effective gain was found to be the product of the RMS values of roll and pitch from the observation period. The penalty function takes the form of 


$$
P_{L P I}=p_{R M S} q_{R M S} \frac{1}{2}\left(\left|\frac{q}{q_{\max }}\right|+\left|\frac{p}{p_{\max }}\right|\right),
$$

where $q_{\max }$ and $p_{\max }$ are the thresholds values for pitch and roll respectively. If the roll and pitch RMS values are found to be less than 1, they will be set to 1 to not lower the value of the penalty function, abiding by the piece-wise functions

$$
\begin{aligned}
& p_{R M S}=\left\{\begin{array}{ll}
1, & p_{R M S} \leq 1 \\
p_{R M S}, & p_{R M S}>1
\end{array},\right. \\
& q_{R M S}=\left\{\begin{array}{ll}
1, & q_{R M S} \leq 1 \\
q_{R M S}, & q_{R M S}>1
\end{array} .\right.
\end{aligned}
$$

The final value of the penalty function is also restricted to be greater than 1 such that

$$
P_{L P I}=\left\{\begin{array}{lll}
1 & , & P_{L P I} \leq 1 \\
P_{L P I}, & P_{L P I}>1
\end{array},\right.
$$

which is intended to never decrease the LPI value and only penalize it. Using the penalty function, the final Landing Period Indicator equation is rewritten as

$$
L P I=P_{L P I}\left(G_{1} E I+G_{2} \dot{E I}\right) .
$$

Figure 4.10 displays the value of the LPI with the penalty function where values of the LPI are the blue solid lines, and the opportune landing times are the red boxes, the LPI system will output a Go signal if the LPI value is less than 1. The penalty function significantly changes the scale between safe and unsafe landing times, improving the effectiveness of the LPI system. The final step for preparing the LPI system for an on-line implementation was to disable the system from outputting a Go signal immediately after a high LPI value was observed. A time delay function 


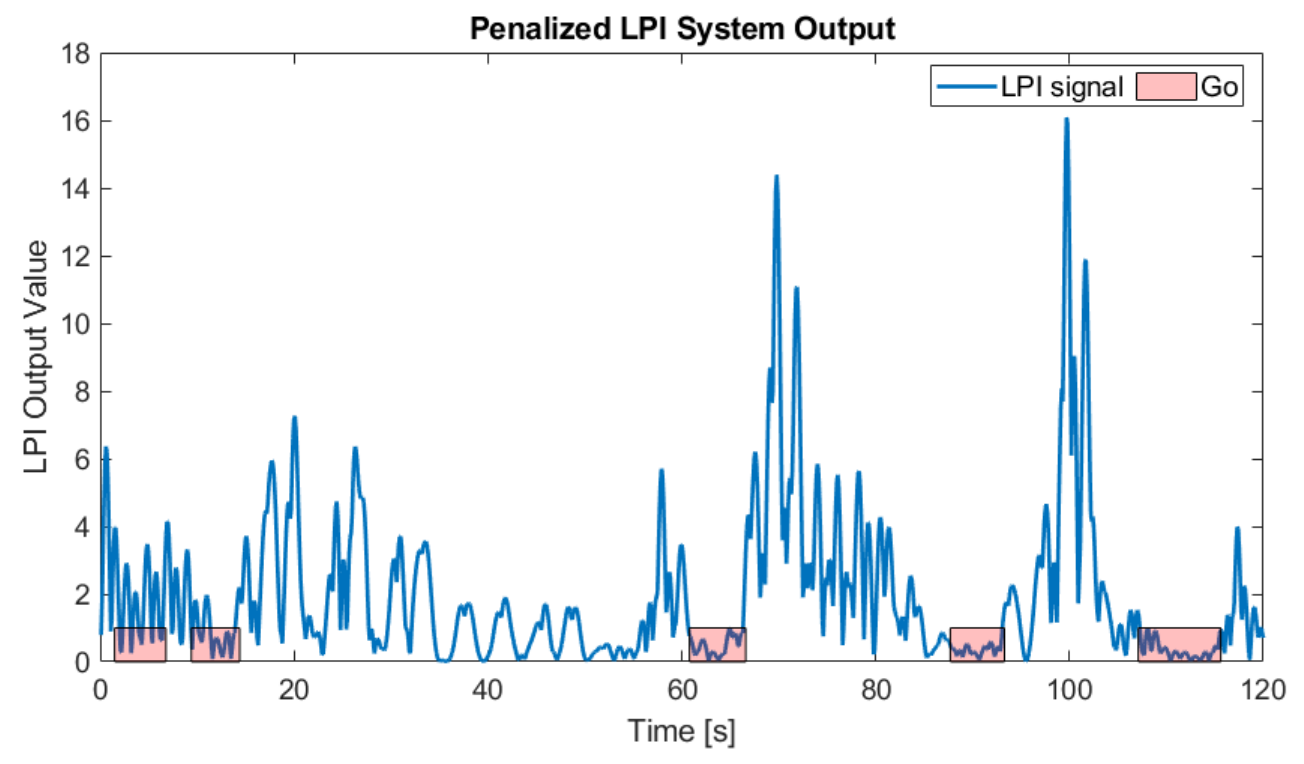

Figure 4.10: Landing period indicator with penalty function.

was implemented to not allow the system to indicate it is safe to land directly after a high LPI value is observed. What is essentially a timer, the delay function decreases the likelihood of a false LPI signal from occurring during dips in the LPI signal which may occur during ship velocity direction reversal.

Figure 4.11 is the Simulink block code use for on-line implementation of the LPI within the simulation. The derivative of the ship roll, pitch, and heave ascertained from the ship pose detection algorithm are taken using the discrete filtered derivative blocks. The ship's heave, roll, and heave velocities are squared then normalized using the coefficients $N_{w}, N_{q}, N_{r}$ respectively, following the form of Equation 4.18. Next, the energy index EI is calculated by averaging the sum of the normalized values. The energy index's derivative is taken using a filtered derivative block, made absolute value, and normalized with coefficient $N_{D E I}$ as written in Equation 4.19. Weighting gains $G_{1}$ and $G_{2}$ are applied to $E I$ and $\dot{E} I$ respectively and their values are summed forming the unpenalized LPI, Equation 4.20. The value of the penalty function is found using the current ship motion multiplied by the RMS values of the roll and pitch found during initialization as described in Equation 4.21. The penalized $L P I$ value is 


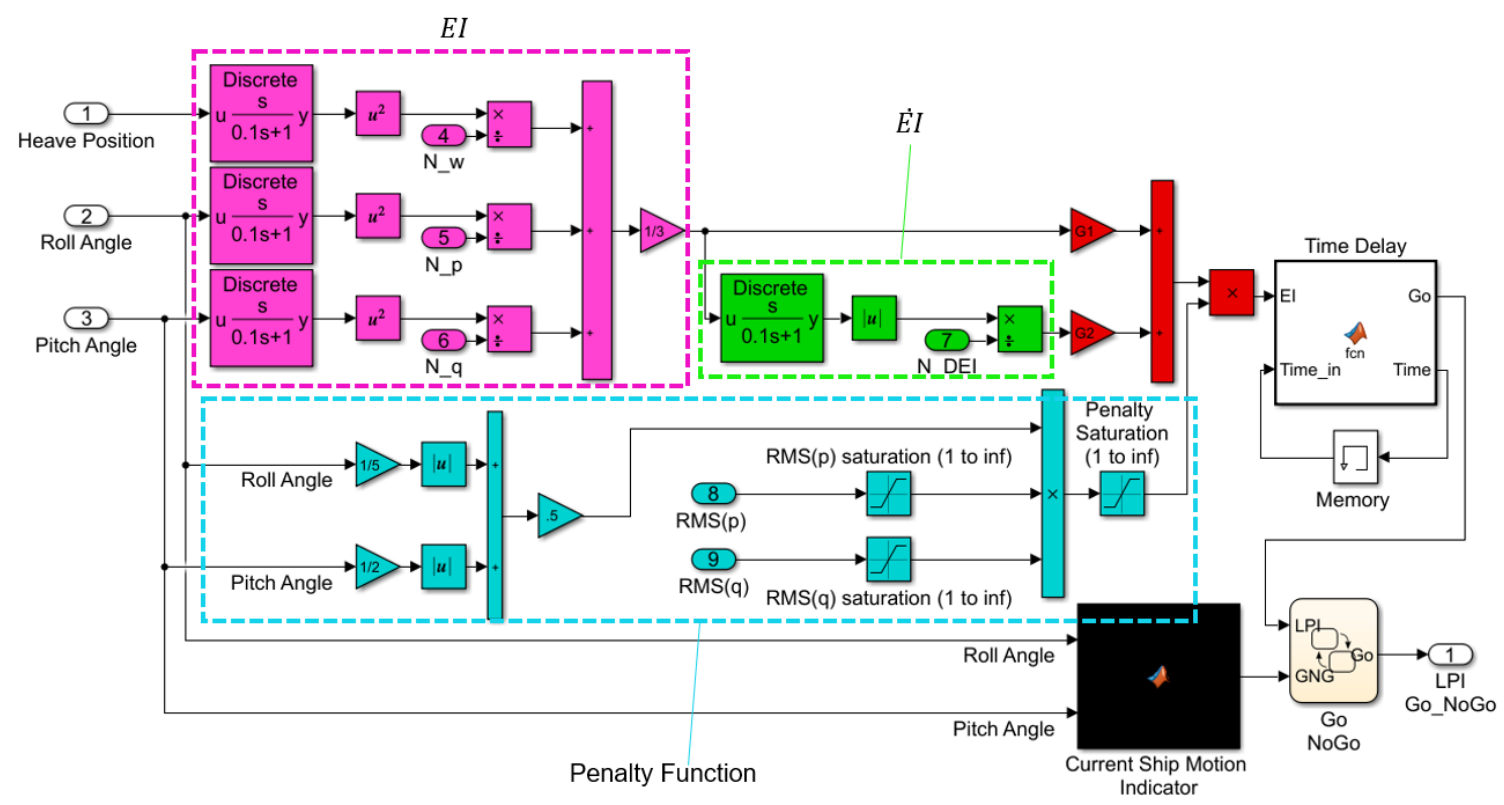

Figure 4.11: Simulink block structure for real time implementation of LPI.

found by taking the product of the penalty function and the unpenalized $L P I$ value corresponding to Equation 4.25. Finally, the LPI signal enters the delay function which will not allow the LPI system to output a Go command immediately after a high energy value is detected, a delay of 1 s performed satisfactorily. For landing, the UAV compares the LPI output to the current ship motion, if both indicate it is safe to land the UAV proceeds to land and will only output NoGo if the LPI reading indicates NoGo and the current ship motion is outside the threshold limits. With the LPI system developed, its effectiveness in determine Go states may be examined.

\subsubsection{Comparison of LPI and SPA}

A study was conducted to evaluate the performance of the LPI to the SPA. The LPI and the SPA were both given the same set of ship motion and 120 s to initialize. The Go signals from both systems were then evaluated for the remainder of the $440 \mathrm{~s}$ of ship motion against the ideal Go times lasting for more than $5 \mathrm{~s}$. The results from the study also include the amount of time each system displayed a Go signal for at 
least $3 \mathrm{~s}$ and how often a false Go signal was indicated. The number of state changes between a Go and NoGo command is also reported. In total there were 14 sets of data used, ranging from sea states $2-6$ and headings of $30^{\circ}, 60^{\circ}$ and $90^{\circ}$. Table 4.2 outlines the efficiencies of the LPI and SPA, where efficiency is deemed the percentage of time the systems correctly identified the sustained Go signals to how often they were on, and was calculated for $5 \mathrm{~s}$ and $3 \mathrm{~s}$ of sustained Go time. The average efficiency for each system is presented at the bottom of the table. The overall performance of each system are remarkably similar, for $5 \mathrm{~s}$, the LPI and SPA had efficiencies of $56 \%$ and $59 \%$ respectively, while, at $3 \mathrm{~s}$, LPI and SPA had respective efficiencies of $70 \%$ and $72 \%$. The average number of state changes from Go to NoGo was also similar, 25 to 21 for the LPI to the SPA respectively. For trial 15, the ship motion did not sustain a Go condition for longer than $5 \mathrm{~s}$. From these results it is expected that the results of using the LPI on the UAV will be similar to using the SPA.

In this thesis the LPI was tuned offline for all 105 sets of ship motion, using the first $120 \mathrm{~s}$ of the corresponding ship data. The tuning factors $N_{w}, N_{q}, N_{r}, p_{R M S}$, and $q_{R M S}$ were imported into the work space for each simulation. In the simulation, once the UAV reached the ship it would loiter for $120 \mathrm{~s}$ to emulate training, and then would proceed to its low hover for landing. To test the various landing methodologies from this chapter, a flight controller state machine is implemented. 
Table 4.2: Efficiencies and state changes for LPI system and SPA

\begin{tabular}{|c|c|c|c|c|c|c|c|c|}
\hline Trial & $\begin{array}{c}\text { Sea } \\
\text { State }\end{array}$ & $\begin{array}{c}\text { Heading } \\
\text { [Deg] }\end{array}$ & $\begin{array}{c}\text { LPI eff } \\
5 \mathrm{~s}\end{array}$ & $\begin{array}{c}\text { SPA eff } \\
5 \mathrm{~s}\end{array}$ & $\begin{array}{c}\text { LPI eff } \\
3 \mathrm{~s}\end{array}$ & $\begin{array}{c}\text { SPA eff } \\
3 \mathrm{~s}\end{array}$ & $\begin{array}{c}\text { Go State } \\
\text { Change } \\
\text { LPI }\end{array}$ & $\begin{array}{c}\text { Go State } \\
\text { Change } \\
\text { SPA }\end{array}$ \\
\hline 1 & \multirow{3}{*}{2} & 30 & 1.00 & 1.00 & 1.00 & 1.00 & 1 & 1 \\
\hline 2 & & 60 & 1.00 & 1.00 & 1.00 & 1.00 & 1 & 1 \\
\hline 3 & & 90 & 1.00 & 1.00 & 1.00 & 1.00 & 2 & 1 \\
\hline 4 & \multirow{3}{*}{3} & 30 & 0.91 & 0.92 & 0.91 & 0.92 & 10 & 20 \\
\hline 5 & & 60 & 0.71 & 0.90 & 0.73 & 0.91 & 24 & 31 \\
\hline 6 & & 90 & 0.63 & 0.75 & 0.68 & 0.81 & 44 & 32 \\
\hline 7 & \multirow{3}{*}{4} & 30 & 0.47 & 0.63 & 0.66 & 0.77 & 53 & 40 \\
\hline 8 & & 60 & 0.39 & 0.61 & 0.51 & 0.75 & 46 & 38 \\
\hline 9 & & 90 & 0.62 & 0.51 & 0.84 & 0.67 & 28 & 37 \\
\hline 10 & \multirow{3}{*}{5} & 30 & 0.14 & 0.17 & 0.24 & 0.32 & 44 & 11 \\
\hline 11 & & 60 & 0.18 & 0.24 & 0.53 & 0.62 & 28 & 12 \\
\hline 12 & & 90 & 0.26 & 0.14 & 0.63 & 0.47 & 16 & 22 \\
\hline 13 & \multirow{3}{*}{6} & 30 & null & null & 0.38 & 0.27 & 33 & 19 \\
\hline 14 & & 60 & 0.54 & 0.58 & 0.69 & 0.68 & 20 & 19 \\
\hline 14 & & 90 & 0.48 & 0.41 & 0.73 & 0.61 & 23 & 29 \\
\hline \multicolumn{3}{|c|}{ Average } & 0.56 & 0.59 & 0.70 & 0.72 & 25 & 21 \\
\hline
\end{tabular}

\subsection{Flight Controller}

Flight control law was developed using a state-machine within Simulink. Figure 4.12 is a state flow diagram of the flight controller. In total there are 12 states, labeled A-G, that the flight controller may enter, however only a single state may be active at a time. In block $\mathbf{A}$ the state machine activates with the start of the simulation bringing the UAV from its landed position to its cruising altitude. In this block the UAV is commanded not to move and has a VTOL velocity of $1 \mathrm{~m} / \mathrm{s}$. States will only 
transition from one to another if the specified criteria identified by the text beside the arrows are met. For example, if the UAV reaches or exceeds its cruising altitude altitude the state-machine will transition from state $\mathbf{A}$ to $\mathbf{B}$. Once the UAV reaches its cruising altitude, the UAV enters state $\mathbf{B}$ and begin to track the track the ship. Once the UAV is within $3 \mathrm{~m}$ to $\mathrm{LZ}$, the UAV will transition from its cruising altitude to a high hover position of $15 \mathrm{~m}$ [85]. While in state $\mathbf{C}$ the UAV powers on the LIDARs and begins to relay ship pose data to SPA or LPI system. If the UAV is using the SPA to land, the UAV will transition to a low hover position after the FFT window is reached and the SPA is predicting ship motion. When using the LPI, the UAV will transition to state $\mathbf{D}$ after the $120 \mathrm{~s}$ training period is complete. For the unregulated system, which lands solely based on the current conditions, the UAV will transition to a high hover and immediately proceeded to a low hover position. If at anytime during state $\mathbf{D}$ the UAV is unable to track the ship it will return to its high hover position, if for some reason there is significant distance error between the UAV and the ship the UAV will begin to ascend to its cruising altitude while simultaneously closing the distance to the LZ. In the simulations the UAV never had to transition back to a high a hover from a low hover. While in state $\mathbf{D}$, the UAV is on standby awaiting for a Go command to land. Depending on the simulation parameters, the Go command will either be given by the SPA, the current ship states, or from the LPI. Once a Go command is received the state-machine will transition into either state $\mathbf{E}, \mathbf{F}$, or $\mathbf{G}$. If the UAV is in Mode 1 it will follow state $\mathbf{E}$ flow. In Mode 1 the UAV has a low hover position of $2.5 \mathrm{~m}$, and will descend at a rate of $0.5 \mathrm{~m} / \mathrm{s}$ to the ship deck. For landing, the altitude controller's reference signal is the difference between the current altitude and the LIDAR distance measurement. If a NoGo command is received while landing the UAV will abort and return to its low hover position. However, if the distance to the ship deck is less than $0.5 \mathrm{~m}$, the UAV will enter state $\mathbf{E} 2$ and will ignore an abort command as the assumption is that it will be safer to continue to land rather than 
abort when within half a metre to the ship deck. Once the UAV reaches the deck the state transitions to $\mathbf{H}$ stopping the simulation.

When in Mode 2, the low hover position is $5 \mathrm{~m}$ and the UAV follows state flow $\mathbf{F}$. In $\mathbf{F} 1$ the UAV descends at $2 \mathrm{~m} / \mathrm{s}$ until it is within $3 \mathrm{~m}$ to the ship deck. Transitioning to $\mathbf{F} 2$, the UAV has a downward velocity of $1 \mathrm{~m} / \mathrm{s}$ until it is within $1 \mathrm{~m}$ of the deck where it will enter state F3. In F3 the UAV will descend at $0.5 \mathrm{~m} / \mathrm{s}$. If at anytime in states F1-F3 a NoGo signal is received the UAV will abort landing and return the low hover position. Within $0.5 \mathrm{~m}$ to the deck the UAV enters $\mathbf{F} 4$ and will always continue to land regardless of a NoGo signal.

If the Mode is set to 3, the UAV uses the active heave compensation system for landing. After a Go signal is received to land the state transitions to G. While landing in Mode 3 the UAV's position and velocity is set by the AHC outlined in Section 4.2. Similar to states $\mathbf{E}$ and $\mathbf{F}$, the UAV will will not abort landing after it is within $0.5 \mathrm{~m}$ to the deck. Once landed, the state changes to $\mathbf{F}$ and the simulation ends. The completion of the state machine flight controller concludes the model. The UAV will now be simulated to track and land on a ship for all ship motion sets and landing methods. 


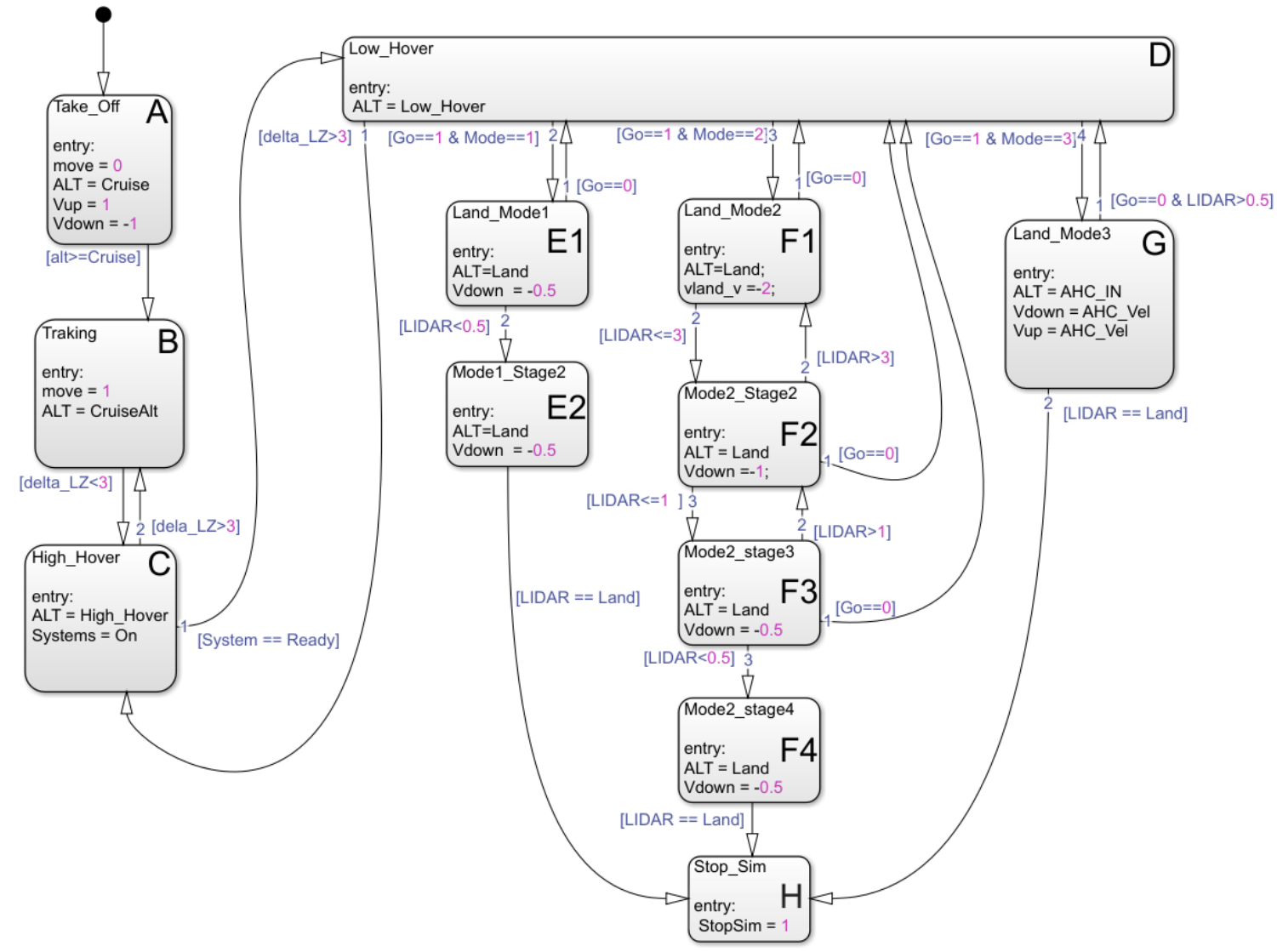

Figure 4.12: State machine flight controller

\subsection{Summary}

In this section the SPA used to determine landing Go times was introduced. The SPA was used to develop an AHC system, a contribution of the thesis, to improve the low hover and landing performance of the UAV. Another contribution within this chapter was the development of a self-training LPI system that improves landing performance without the use of signal prediction. Finally, the state-machine flight controller for autonomous flight and landing was described

In the the following chapter, the results of using the LPI, SPA, and AHC system for landing the UAV are presented. 


\section{Chapter 5}

\section{Results}

To test if the SPA, LPI and AHC will improve the landing performance of a quad rotor UAV a series of batch simulations were performed. A summary off all the testing conditions are highlighted in Table 5.1. Each test case was performed twice, once with a Low Hover (LH) of $2.5 \mathrm{~m}$ and second with a $\mathrm{LH}$ of $5 \mathrm{~m}$. When landing from a $2.5 \mathrm{~m}$ $\mathrm{LH}$, the UAV used a $0.5 \mathrm{~m} / \mathrm{s}$ landing velocity and when landing from a $5 \mathrm{~m} \mathrm{LH}$, the UAV followed a piece-wise landing velocity given in Equation 4.14. However, if using AHC, the AHC system will determine the landing trajectory for the UAV. Test 1, the unregulated control, had the UAV land if the current ship motion was below the roll and pitch threshold limits. Test 2 used the SPA to predict when the roll and pitch values would be below their respective thresholds for at least 5 seconds and landed the UAV accordingly. For test 3, the SPA was used to determine Go states for landing and the AHC was used to assist the UAV in maintaining a minimum low hover position above the ship and to plan landing trajectories. During Test 4, the LPI described in Section 4.3 was used to determine the Go and NoGo commands for landing. Test 5 combined the LPI and SPA, where the UAV would land if a Go state was determined by the SPA and the LPI. Case 6 investigated the effectiveness of using the AHC with the LPI and the SPA. Finally, case 7 used the LPI to determine 
Go states and the AHC to plan landing trajectories.

Table 5.1: Ship Landing Test Cases

\begin{tabular}{|l|l|}
\hline Test Case & Description \\
\hline 1 & No SPA \\
\hline 2 & $\mathrm{SPA}$ \\
\hline 3 & $\mathrm{SPA}+\mathrm{AHC}$ \\
\hline 4 & $\mathrm{LPI}$ \\
\hline 5 & $\mathrm{LPI}+\mathrm{SPA}$ \\
\hline 6 & $\mathrm{LPI}+\mathrm{SPA}+\mathrm{AHC}$ \\
\hline 7 & $\mathrm{LPI}+\mathrm{AHC}$ \\
\hline
\end{tabular}

During each simulation the UAV was set to take off, track, and land on a ship for various types of ship motion. Table 5.2 is a summary of all possible ship motion combinations that was presented in Section 3.7. The sea states ranged from calm, sea state 2 , to very rough, sea state 6 , and the heading of the ship varied from $0^{\circ}$ to $180^{\circ}$ in increments of $30^{\circ}$. The wind direction was also changed to match the heading of the ship. For each heading and sea state, the ship was modeled traveling at speeds of 6, 8 and $10 \mathrm{kn}$. In total, there were 105 sets of ship motion used to test each landing method. Wind gusts were saturated at $10 \mathrm{~m} / \mathrm{s}$ as per the analysis in Section 3.6.

Table 5.2: Ship Motion Combinations

\begin{tabular}{|l|l|}
\hline Ship motion Variables & Range \\
\hline Sea State & 23456 \\
\hline Ship Velocity (kn) & 6810 \\
\hline Ship Heading (deg) & 0306090120150180 \\
\hline Total Runs & 105 \\
\hline
\end{tabular}




\subsection{Summary of Results}

A landing is deemed successful if the UAV landed when the ship's pitch was less than $2^{\circ}$, roll less than $5^{\circ}$ and with a relative impact velocity less than or equal to $1 \mathrm{~m} / \mathrm{s}$. For each batch of simulations, the number of UAVs which did not land or landed within a NoGo state were recorded. In this section, the average number of times the flight controller changed from a Go to a NoGo state is also reported. Table 5.3 is a summary of all the results from the $2.5 \mathrm{~m} \mathrm{LH}$ test cases. Using the LPI, SPA and AHC all improved the landing performance of the UAV, and combining the LPI, SPA and AHC resulted in the highest number of successful landings. The UAV that landed using the AHC system during Go states agreed on by the LPI and SPA $(\mathrm{LPI}+\mathrm{SPA}+\mathrm{AHC})$ had 93 successful landings. From the remaining 12 failed landings, 8 were due to the UAV landing in periods of high ship motion and 6 were from high impact velocities, meaning that there was an overlap of 2 trials which failed to land within the motion thresholds and velocity limit. For the LPI+SPA+AHC case, 7 of the failed landings occurred during a NoGo condition indicating that either the UAV was intercepted by the ship while attempting to maintain a low hover or subsequent to an aborted landing, although the later was more frequently observed when using AHC. The average number of state changes between Go and NoGo states were all similar between the trials, although the unregulated UAV and the UAV using AHC each had one more landing attempt on average than the other landing methods. The landing systems which did not use signal prediction for determining Go states, i.e the unregulated, LPI and LPI+AHC UAVs, had the least number of landings occur during NoGo states, however, that did not correlate to an increase in the total number of successful landings.

The UAV using the LPI for landing had improved results compared to the unregulated UAV, however, all simulations using signal prediction outperformed the standard LPI system for the $2.5 \mathrm{~m}$ LH cases. It was observed that pairing the LPI+AHC 
resulted in the least number of landings with high impact velocities. Based off the results in Table 5.3 the ranking from best performing to worst performing systems for landing a UAV from a $2.5 \mathrm{~m} \mathrm{LH}$ are:

- $\mathrm{LPI}+\mathrm{SPA}+\mathrm{AHC}$

- $\mathrm{SPA}+\mathrm{AHC}$

- $\mathrm{LPI}+\mathrm{SPA}$

- $\mathrm{LPI}+\mathrm{AHC}$

- SPA

- LPI

- Unregulated

Table 5.3: Executive summary of $2.5 \mathrm{~m}$ low hover results.

\begin{tabular}{|l|c|c|c|c|c|c|}
\hline Landing & $\begin{array}{c}\text { Successful } \\
\text { Landing } \\
(\mathbf{1 0 5})\end{array}$ & $\begin{array}{c}\text { Failed to Land } \\
\text { within Roll } \\
\text { or Pitch Limit }\end{array}$ & $\begin{array}{c}\text { Exceeded } \\
\text { Impact Velocity } \\
\text { Limit }\end{array}$ & $\begin{array}{c}\text { UAV } \\
\text { did not } \\
\text { Land }\end{array}$ & $\begin{array}{c}\text { Landed NoGo } \\
\text { in } \\
\text { State }\end{array}$ & $\begin{array}{c}\text { Avg. Landing } \\
\text { State Change } \\
\text { (NoGo-Go) }\end{array}$ \\
\hline Unregulated & 70 & 16 & 28 & 0 & 4 & 3 \\
\hline SPA & 82 & 11 & 18 & 0 & 11 & 2 \\
\hline SPA+AHC & 87 & 8 & 12 & 0 & 9 & 3 \\
\hline LPI & 79 & 19 & 24 & 0 & 4 & 2 \\
\hline LPI+SPA & 87 & 12 & 15 & 0 & 9 & 2 \\
\hline LPI+SPA+AHC & 93 & 8 & 6 & 0 & 7 & 2 \\
\hline LPI+AHC & 87 & 16 & 6 & 0 & 4 & 2 \\
\hline
\end{tabular}

Table 5.4 highlights the executive results for the $5 \mathrm{~m} \mathrm{LH}$ landing cases. Similar to the the $2.5 \mathrm{~m} \mathrm{LH}$ results, the UAV using $\mathrm{LPI}+\mathrm{SPA}+\mathrm{AHC}$ outperformed all other landing methods. Using the LPI+SPA+AHC, 94 out of the 105 trials had successful landings with 1 failed landing due to ship motion and 1 due to impact velocity, during this test case $9 \mathrm{UAVs}$ did not land within the $600 \mathrm{~s}$ simulation. The UAV using LPI+AHC also had 94 successful simulations, however, all 105 UAV landed, resulting in greater number of unsafe landings. For the $5 \mathrm{~m} \mathrm{LH}$ simulations, every landing method which used an SPA to determine Go landing states had UAVs that 
did not land, which is not unexpected, as there are sets of ship motion that do not have 5 s of consecutive Go time, and the SPAs are tuned for 5 s prediction.

Overall, every UAV incorporating either the SPA or LPI outperformed the unregulated UAV. It was observed that the UAVs utilizing the SPA had a significant increase in the number of landings within the ship motion thresholds than those that did not. The systems utilizing AHC also had significantly lower unsafe landings due to impact velocities. The LPI was found to improve UAV landing performance compared to the unregulated system, however, when not combined with either the SPA or AHC system, the results were still poor in comparison to the other systems using SPA and AHC. Similar to the $2.5 \mathrm{~m}$ LH cases, the state changes from Go to NoGo were all within range of each other, with the LPI having the least number of state changes. For the $5 \mathrm{~m}$ LH cases, the unregulated system not only performed the worst, but was also the only case with a high number of landings during NoGo conditions. The UAV using the SPA had 2 cases during sea state 6 where the UAV lost stability and crashed due to rapid landing state changes and violent ship movement which overwhelmed the UAV's control efforts. The overall ranking for best performing to worst performing landing systems for the $5 \mathrm{~m} \mathrm{LH}$ test cases are:

- $\mathrm{LPI}+\mathrm{SPA}+\mathrm{AHC}$

- $\mathrm{LPI}+\mathrm{AHC}$

- $\mathrm{SPA}+\mathrm{AHC}$

- $\mathrm{LPI}+\mathrm{SPA}$

- SPA

- LPI

- Unregulated 
Table 5.4: Executive summary of $5 \mathrm{~m}$ low hover results.

\begin{tabular}{|c|c|c|c|c|c|c|}
\hline $\begin{array}{l}\text { Landing } \\
\text { System }\end{array}$ & $\begin{array}{l}\text { Successful } \\
\text { Landing } \\
\text { (105) }\end{array}$ & $\begin{array}{l}\text { Failed to Land } \\
\text { within Roll } \\
\text { or Pitch Limit }\end{array}$ & $\begin{array}{c}\text { Exceeded } \\
\text { Impact Velocity } \\
\text { Limit }\end{array}$ & $\begin{array}{c}\text { UAV } \\
\text { did not } \\
\text { Land }\end{array}$ & $\begin{array}{c}\text { Landed } \\
\text { in NoGo } \\
\text { State }\end{array}$ & $\begin{array}{c}\text { Avg. Landing } \\
\text { State Change } \\
\text { (NoGo-Go) }\end{array}$ \\
\hline Unregulated & 70 & 20 & 31 & 0 & 12 & 4 \\
\hline SPA & 85 & 4 & 13 & $5^{*}$ & 0 & 3 \\
\hline $\mathrm{SPA}+\mathrm{AHC}$ & 87 & 3 & 7 & 8 & 0 & 4 \\
\hline LPI & 78 & 19 & 27 & 0 & 1 & 2 \\
\hline $\mathrm{LPI}+\mathrm{SPA}$ & 85 & 5 & 10 & 5 & 1 & 4 \\
\hline $\mathrm{LPI}+\mathrm{SPA}+\mathrm{AHC}$ & 94 & 1 & 1 & 9 & 0 & 3 \\
\hline $\mathrm{LPI}+\mathrm{AHC}$ & 94 & 11 & 6 & 0 & 1 & 3 \\
\hline
\end{tabular}

\subsection{Results - Low Hover, $2.5 \mathrm{~m}$}

A detailed summary of the UAV's landing performance in each sea state using the various landing systems is provided in Table 5.5. The $2.5 \mathrm{~m} \mathrm{LH}$ trials all had a $100 \%$ success rate for landing in sea states 2 and 3, except when using the LPI, which had 1 case where the ship motion threshold and impact velocity limit were exceeded. The UAV using LPI+SPA was the only landing system with a $100 \%$ success rate in sea state 4, followed by the LPI and LPI+SPA+AHC systems, which each had 19 out of 21 successful landings. There was a sharp decline in landing performance for all test cases in sea state 5 , with the UAVs landing with LPI+SPA and LPI+SPA+AHC having the best performances, each having 14 successful landings; the other regulated systems were up to 2 successful landings behind, whereas the unregulated system had only 8 successful landings in sea state 5 . Interestingly, all the regulated landing systems that used the AHC system had more successful landings in sea sate 6 than in sea state 5, whereas, the majority of the other systems saw a steady decline in the number of successful landings. The improvement in landing performance in sea state 6 for the UAVs utilizing the AHC system may be attributed to a shorter landing 
distance due to the larger heave amplitudes. Overall there is a correlation between the increase in sea state and the total number of failures due to ship motion and impact velocities as well as a general rise in the number of landing state changes and landings during NoGo conditions.

Table 5.5: Summary of $2.5 \mathrm{~m}$ low hover results.

\begin{tabular}{|c|c|c|c|c|c|c|c|}
\hline $\begin{array}{l}\text { Landing } \\
\text { System }\end{array}$ & $\begin{array}{c}\text { Sea } \\
\text { State }\end{array}$ & $\begin{array}{l}\text { Successful } \\
\text { Landing } \\
(21)\end{array}$ & $\begin{array}{l}\text { Failed to Land } \\
\text { within Roll or } \\
\text { Pitch Limit }\end{array}$ & $\begin{array}{c}\text { Exceeded } \\
\text { Impact Velocity } \\
\text { Limit }\end{array}$ & $\begin{array}{c}\text { UAV } \\
\text { did not } \\
\text { Land }\end{array}$ & $\begin{array}{c}\text { Landed } \\
\text { in NoGo } \\
\text { State }\end{array}$ & $\begin{array}{c}\text { Avg. Landing } \\
\text { State Change } \\
\text { (NoGo-Go) }\end{array}$ \\
\hline \multirow{5}{*}{ Unregulated } & 2 & 21 & 0 & 0 & 0 & 0 & 1 \\
\hline & 3 & 21 & 0 & 0 & 0 & 0 & 3 \\
\hline & 4 & 11 & 4 & 6 & 0 & 0 & 4 \\
\hline & 5 & 8 & 6 & 10 & 0 & 1 & 4 \\
\hline & 6 & 9 & 6 & 12 & 0 & 3 & 4 \\
\hline \multirow{5}{*}{ SPA } & 2 & 21 & 0 & 0 & 0 & 0 & 1 \\
\hline & 3 & 21 & 0 & 0 & 0 & 0 & 1 \\
\hline & 4 & 18 & 0 & 3 & 0 & 0 & 4 \\
\hline & 5 & 13 & 4 & 6 & 0 & 4 & 4 \\
\hline & 6 & 9 & 7 & 9 & 0 & 7 & 2 \\
\hline \multirow{5}{*}{$\mathrm{SPA}+\mathrm{AHC}$} & 2 & 21 & 0 & 0 & 0 & 0 & 1 \\
\hline & 3 & 21 & 0 & 0 & 0 & 0 & 2 \\
\hline & 4 & 17 & 1 & 3 & 0 & 1 & 3 \\
\hline & 5 & 13 & 4 & 4 & 0 & 5 & 4 \\
\hline & 6 & 15 & 3 & 5 & 0 & 3 & 3 \\
\hline \multirow{5}{*}{ LPI } & 2 & 21 & 0 & 0 & 0 & 0 & 1 \\
\hline & 3 & 20 & 1 & 1 & 0 & 0 & 1 \\
\hline & 4 & 19 & 1 & 2 & 0 & 0 & 2 \\
\hline & 5 & 11 & 8 & 9 & 0 & 1 & 2 \\
\hline & 6 & 8 & 9 & 12 & 0 & 3 & 2 \\
\hline \multirow{5}{*}{$\mathrm{LPI}+\mathrm{SPA}$} & 2 & 21 & 0 & 0 & 0 & 0 & 1 \\
\hline & 3 & 21 & 0 & 0 & 0 & 0 & 1 \\
\hline & 4 & 21 & 0 & 0 & 0 & 0 & 4 \\
\hline & 5 & 14 & 5 & 5 & 0 & 5 & 3 \\
\hline & 6 & 10 & 7 & 10 & 0 & 7 & 2 \\
\hline \multirow{5}{*}{$\mathrm{LPI}+\mathrm{SPA}+\mathrm{AHC}$} & 2 & 21 & 0 & 0 & 0 & 0 & 1 \\
\hline & 3 & 21 & 0 & 0 & 0 & 0 & 1 \\
\hline & 4 & 19 & 2 & 0 & 0 & 0 & 2 \\
\hline & 5 & 14 & 4 & 3 & 0 & 4 & 2 \\
\hline & 6 & 18 & 2 & 3 & 0 & 3 & 2 \\
\hline \multirow{5}{*}{$\mathrm{LPI}+\mathrm{AHC}$} & 2 & 21 & 0 & 0 & 0 & 0 & 1 \\
\hline & 3 & 21 & 0 & 0 & 0 & 0 & 1 \\
\hline & 4 & 18 & 3 & 0 & 0 & 0 & 2 \\
\hline & 5 & 12 & 8 & 3 & 0 & 1 & 2 \\
\hline & 6 & 15 & 5 & 3 & 0 & 3 & 3 \\
\hline
\end{tabular}

Table 5.6 outlines the the average total landing time, simulation time, impact velocity, and landing angle between the UAV and ship for each landing system using a $2.5 \mathrm{~m} \mathrm{LH}$. The standard deviation for each parameter in Table 5.6 is also given. The landing angle between the UAV and ship deck was not a metric for determining a successful landing, however, a higher landing angle was found to correlate to a higher 
number of unsuccessful landings. The landing angle was not considered a metric for successful landing as the UAV has to articulate its body in order to track the ship, whereas, a helicopter would primarily change the orientation of its swash plate, therefore, it is expected that during periods of high ship motion and strong winds that the UAVs landing angle will be higher. The unregulated UAV had the shortest average landing and simulation times, however had the greatest average landing velocity, and the second greatest average landing angle. All of the systems utilizing the AHC, LPI, or SPA had similar average simulation and landing times, however, the UAVs using the AHC had average landing velocities approximately equal to the goal impact velocity of $0.5 \mathrm{~m} / \mathrm{s}$. The standard deviation for the cases using the AHC were also less than the cases which did not use AHC, indicating that using the AHC leads to more consistent landing impact velocities. The average landing impact velocity and land angle for the UAV using $\mathrm{LPI}+\mathrm{SPA}+\mathrm{AHC}$ were the lowest which corroborates their performance in Table 5.5. Case by case results for all the $2.5 \mathrm{~m}$ landing methods are given in appendix A.

Table 5.6: Average land time, simulation time, impact velocity and land angle for the $2.5 \mathrm{~m}$ low hover test cases.

\begin{tabular}{|c|c|c|c|c|c|}
\hline \multirow{2}{*}{ Land System } & Avg / Std. & $\begin{array}{c}\text { Total Landing } \\
\text { Time }[\mathbf{s}]\end{array}$ & $\begin{array}{c}\text { Simulation } \\
\text { Time }[\mathbf{s}]\end{array}$ & $\begin{array}{c}\text { Impact } \\
\text { Velocity } \\
{[\mathbf{m} / \mathbf{s}]}\end{array}$ & $\begin{array}{c}\text { UAV-Ship } \\
\text { Land Angle } \\
{[\mathbf{d e g}]}\end{array}$ \\
\hline \multirow{2}{*}{ Unregulated } & Avg. & 5.24 & 65.05 & 0.85 & 3.61 \\
\cline { 2 - 6 } & Std. & 3.36 & 56.24 & 0.59 & 2.73 \\
\hline \multirow{2}{*}{ SPA } & Avg. & 8.26 & 207.29 & 0.83 & 4.30 \\
\cline { 2 - 6 } & Std. & 9.32 & 49.68 & 0.55 & 4.02 \\
\hline \multirow{2}{*}{ SPA+AHC } & Avg. & 7.09 & 203.87 & 0.66 & 3.79 \\
\cline { 2 - 6 } & Std. & 11.15 & 63.38 & 0.51 & 2.62 \\
\hline \multirow{2}{*}{ LPI } & Avg. & 6.70 & 185.53 & 0.82 & 4.16 \\
\cline { 2 - 6 } & Std. & 7.49 & 35.76 & 0.53 & 3.73 \\
\hline \multirow{2}{*}{ LPI+SPA } & Avg. & 9.54 & 208.73 & 0.76 & 3.72 \\
\cline { 2 - 6 } & Std. & 12.69 & 69.73 & 0.50 & 2.92 \\
\hline \multirow{2}{*}{ LPI+SPA+AHC } & Avg. & 7.54 & 203.58 & 0.49 & 3.32 \\
\cline { 2 - 6 } & Std. & 9.60 & 64.99 & 0.39 & 2.21 \\
\hline \multirow{2}{*}{ LPI+AHC } & Avg. & 6.16 & 188.57 & 0.52 & 3.36 \\
\cline { 2 - 6 } & Std. & 6.76 & 36.64 & 0.34 & 2.32 \\
\hline
\end{tabular}




\subsection{Results - Low Hover, $5 \mathrm{~m}$}

Table 5.7 highlights the landing results for the $5 \mathrm{~m}$ LH test cases. All landing methods had a $100 \%$ success rate for landing in sea state 2 , and only the UAVs using AHC had $100 \%$ success in sea state 3 . Higher than sea state 3 , the unregulated UAV had a sharp decline in landing performance, whereas the other systems had a more gradual decline. Only the UAVs using LPI+AHC and LPI+AHC+SPA had a 100\% landing success rate in sea state 4, and the UAV using LPI+SPA was one off from perfect. The highest performing system in sea state 5 was LPI+AHC, which had 15 successful landings. During sea state 5 there were multiple instances where the UAVs using a SPA were unable to land, this could be corrected by shortening the SPA's prediction horizon to accommodate for the shorter intervals of safe landing times. The results in Table 5.7 indicate that using the SPA leads to safer UAV operation, however, the potential inability to land in higher sea states needs to be addressed. Further, the only UAVs that crashed during any of the simulations were using the SPA. The LPI+AHC was found to be the most successful landing system in sea state 5, having landed 15 UAVs safely. The top performing UAV in sea state 6 used the LPI+AHC+SPA, where 18/21 UAVs landed successfully. Similar to the $2.5 \mathrm{~m} \mathrm{LH}$ cases, there was a correlation between an increase in the number of state changes and failed landings to the increase in sea state.

Table 5.8 outlines the average values and accompanying standard deviations of the total landing time, simulation time, impact velocity and UAV-ship landing angle for

the $5 \mathrm{~m}$ LH test cases. The results reflect the observations made for Table 5.6, where the unregulated UAV had the shortest landing and simulation times. The unregulated system also had the highest average impact velocity and average largest landing angle compared to the other systems. The UAVs using the AHC system also had similar trends to those found in Table 5.6, where the average landing impact velocities using 
Table 5.7: Summary of $5 \mathrm{~m}$ low hover results.

\begin{tabular}{|c|c|c|c|c|c|c|c|}
\hline $\begin{array}{l}\text { Landing } \\
\text { System }\end{array}$ & $\begin{array}{c}\text { Sea } \\
\text { State }\end{array}$ & $\begin{array}{l}\text { Successful } \\
\text { Landing } \\
(21)\end{array}$ & $\begin{array}{l}\text { Failed to Land } \\
\text { within Roll or } \\
\text { Pitch Limit }\end{array}$ & $\begin{array}{c}\text { Exceeded } \\
\text { Impact Velocity } \\
\text { Limit }\end{array}$ & $\begin{array}{c}\text { UAV } \\
\text { did not } \\
\text { Land }\end{array}$ & $\begin{array}{c}\text { Landed } \\
\text { in NoGo } \\
\text { State }\end{array}$ & $\begin{array}{c}\text { Avg. Landing } \\
\text { State Change } \\
\text { (NoGo-Go) }\end{array}$ \\
\hline \multirow{5}{*}{ Unregulated } & 2 & 21 & 0 & 0 & 0 & 0 & 1 \\
\hline & 3 & 19 & 0 & 2 & 0 & 0 & 1 \\
\hline & 4 & 15 & 4 & 5 & 0 & 3 & 3 \\
\hline & 5 & 7 & 9 & 12 & 0 & 6 & 9 \\
\hline & 6 & 8 & 7 & 12 & 0 & 3 & 6 \\
\hline \multirow{5}{*}{ SPA } & 2 & 21 & 0 & 0 & 0 & 0 & 1 \\
\hline & 3 & 21 & 0 & 0 & 0 & 0 & 1 \\
\hline & 4 & 17 & 0 & 4 & 0 & 0 & 3 \\
\hline & 5 & 12 & 2 & 4 & 3 & 0 & 5 \\
\hline & 6 & 14 & 2 & 5 & $2^{*}$ & 0 & 6 \\
\hline \multirow{5}{*}{$\mathrm{SPA}+\mathrm{AHC}$} & 2 & 21 & 0 & 0 & 0 & 0 & 1 \\
\hline & 3 & 21 & 0 & 0 & 0 & 0 & 2 \\
\hline & 4 & 16 & 3 & 2 & 0 & 0 & 3 \\
\hline & 5 & 14 & 0 & 1 & 6 & 0 & 7 \\
\hline & 6 & 15 & 0 & 4 & 2 & 0 & 8 \\
\hline \multirow{5}{*}{ LPI } & 2 & 21 & 0 & 0 & 0 & 0 & 1 \\
\hline & 3 & 18 & 3 & 3 & 0 & 1 & 1 \\
\hline & 4 & 14 & 5 & 7 & 0 & 0 & 2 \\
\hline & 5 & 14 & 6 & 7 & 0 & 0 & 3 \\
\hline & 6 & 11 & 5 & 10 & 0 & 0 & 3 \\
\hline \multirow{5}{*}{$\mathrm{LPI}+\mathrm{SPA}$} & 2 & 21 & 0 & 0 & 0 & 0 & 1 \\
\hline & 3 & 20 & 0 & 0 & 0 & 0 & 1 \\
\hline & 4 & 20 & 1 & 0 & 0 & 0 & 3 \\
\hline & 5 & 13 & 1 & 2 & 5 & 0 & 4 \\
\hline & 6 & 11 & 3 & 8 & 0 & 1 & 4 \\
\hline \multirow{5}{*}{$\mathrm{LPI}+\mathrm{SPA}+\mathrm{AHC}$} & 2 & 21 & 0 & 0 & 0 & 0 & 1 \\
\hline & 3 & 21 & 0 & 0 & 0 & 0 & 1 \\
\hline & 4 & 21 & 0 & 0 & 0 & 0 & 3 \\
\hline & 5 & 13 & 1 & 0 & 7 & 0 & 5 \\
\hline & 6 & 18 & 0 & 1 & 2 & 0 & 5 \\
\hline \multirow{5}{*}{$\mathrm{LPI}+\mathrm{AHC}$} & 2 & 21 & 0 & 0 & 0 & 0 & 1 \\
\hline & 3 & 21 & 0 & 0 & 0 & 0 & 1 \\
\hline & 4 & 21 & 0 & 0 & 0 & 0 & 3 \\
\hline & 5 & 15 & 6 & 4 & 0 & 1 & 5 \\
\hline & 6 & 16 & 5 & 2 & 0 & 0 & 4 \\
\hline
\end{tabular}

the AHC were all lower than the other methods. The average landing velocities found for the UAVs using the AHC did not meet the goal of $0.5 \mathrm{~m} / \mathrm{s}$ however they were still below the maximum value of $1 \mathrm{~m} / \mathrm{s}$ and on average had the lowest standard deviation for impact velocity. Although the UAV using LPI+SPA+AHC had the best overall landing performance, it also had the longest average simulation time, with the greatest standard deviation. A correlation was not found between average landing and simulation time to overall landing performance. Appendix B contains the final landing result values for each $5 \mathrm{~m}$ low hover case. 
Table 5.8: Average land time, simulation time, impact velocity and land angle for the $5 \mathrm{~m}$ low hover test cases.

\begin{tabular}{|c|c|c|c|c|c|}
\hline \multirow{2}{*}{ Land System } & Avg. / Std. & $\begin{array}{c}\text { Total Landing } \\
\text { Time }[\mathbf{s}]\end{array}$ & $\begin{array}{c}\text { Simulation } \\
\text { Time }[\mathbf{s}]\end{array}$ & $\begin{array}{c}\text { Impact } \\
\text { Velocity } \\
{[\mathbf{m} / \mathbf{s}]}\end{array}$ & $\begin{array}{c}\text { UAV-Ship } \\
\text { Land Angle } \\
{[\mathbf{d e g}]}\end{array}$ \\
\hline \multirow{2}{*}{ Unregulated } & Avg. & 9.79 & 67.22 & 1.35 & 7.77 \\
\cline { 2 - 6 } & Std. & 14.98 & 60.90 & 1.66 & 13.71 \\
\hline \multirow{2}{*}{ SPA } & Avg. & 16.58 & 235.48 & 0.76 & 3.59 \\
\cline { 2 - 6 } & Std. & 23.47 & 107.02 & 0.66 & 2.33 \\
\hline \multirow{2}{*}{ SPA+AHC } & Avg. & 15.56 & 247.15 & 0.65 & 2.99 \\
\cline { 2 - 6 } & Std. & 20.61 & 132.69 & 0.57 & 1.76 \\
\hline \multirow{2}{*}{ LPI } & Avg. & 10.59 & 190.08 & 0.83 & 4.26 \\
\cline { 2 - 6 } & Std. & 13.47 & 49.79 & 0.43 & 3.47 \\
\hline \multirow{2}{*}{ LPI+SPA } & Avg. & 17.41 & 241.87 & 0.74 & 3.66 \\
\cline { 2 - 6 } & Std. & 24.36 & 125.80 & 0.60 & 3.92 \\
\hline \multirow{2}{*}{ LPI+SPA+AHC } & Avg. & 15.72 & 256.34 & 0.64 & 2.91 \\
\cline { 2 - 6 } & Std. & 19.06 & 138.22 & 0.19 & 1.67 \\
\hline \multirow{2}{*}{ LPI+AHC } & Avg. & 12.46 & 205.74 & 0.71 & 3.12 \\
\cline { 2 - 6 } & Std. & 16.90 & 65.15 & 0.26 & 2.15 \\
\hline
\end{tabular}

\subsection{Discussion}

From the given results it is clear that regulating the UAVs landing using the methods described in this thesis enhance UAV landing performance. Both the LPI and SPA were effective in modulating Go states, however, were most effective when paired with each other. The AHC was found to significantly reduce the impact velocity between the UAV and ship. For the $2.5 \mathrm{~m}$ LH case, the reduction in NoGo landings between the SPA and SPA+AHC case is indicative that the AHC was effective in reducing ship-UAV interceptions.

The overall best landing performance for both LH cases was when the LPI, SPA, and AHC were combined with each other. Therefore, it would be recommended to implement the LPI, SPA and AHC on a UAV. However, each system has its pros and cons in certain applications. For instance, the non-regulated system is best suited for landing during sea states 3 and below. Not only was the landing success rate the same as the regulated systems, but on average the UAV was able to land within the first $66 \mathrm{~s}$ of the simulation, whereas, the other methods had a cumulative average of $215 \mathrm{~s}$ 
simulations. In higher sea states, using the LPI or the SPA would be advantageous. The SPA showed overall better performance than the LPI, however, there were cases for the $5 \mathrm{~m} \mathrm{LH}$ where when using the SPA, the UAV was unable to land. The UAV being unable to land may be viewed as positive or negative. That is to say, it can be argued that not landing at all is better than landing unsafely, however, there may be times when it might be more dangerous to loiter above the ship deck in adverse conditions than it would be to attempt a landing. Therefore, it may be useful to have a self-tuning SPA that adapts the prediction horizon based off past ship motion trends. Although the LPI performed worse than the SPA, it could potentially be developed further to better estimate Go states. An advantage of the LPI is its simple implementation and computational efficiency and effectiveness for a non-predictive system. The LPI would be suitable as a stand alone landing system up to sea state 4, whereafter, the decreased forecast performance may endanger the UAV or deck crew. From the results, it would not be recommended to use the SPA as a standalone system above sea states 4 , however, if forced to land during higher sea states, using the SPA will drastically increase the potential of a safe landing. The AHC can be used in any sea state, however, it is most recommended to be used in any sea state greater than 3. As stated, combining the LPI, SPA, and AHC significantly increases the likelihood of a successful landing, it is recommended to be used in any sea state greater than 3. However, a rotorcraft should always attempt to land within the recommended SHOLs, but if forced to land in hazardous conditions, using the LPI+SPA+AHC could potentially avert dangerous scenarios. 


\section{Chapter 6}

\section{Conclusions and Future Work}

The main objectives of this thesis were to:

1. Develop a simulator for autonomously landing an Unnamed Aerial Vehicle (UAV) on a maritime vessel

2. Integrate the Signal Prediction Algorithm (SPA) to improve UAV landing performance

3. Evaluate the performance of the landing systems

The key contributions of this thesis were:

1. Using the SPA to develop an Active Heave Compensation (AHC) system for:

- maintaining a safe low hover position above a ship deck

- landing the UAV with a goal impact velocity

2. The development of a self-tuning Landing Period Indicator (LPI) to improve landing performance without the use of signal prediction

3. A portion of this research has been presented and published in the proceedings of the IEEE Oceans 18 Conference [9] 
The first objective of this thesis, developing a simulator, was achieved through using Simulink to develop the actuation and control of a modeled UAV. The fidelity of the synthetic environment was enhanced by introducing wind gusts and atmospheric turbulence to perturb the UAV. LIDARs were emulated and added to the UAV for aerial ship pose detection.

The second objective of this thesis was to integrate the SPA to improve UAV landing performance. This objective was accomplished through using a LIDAR-based ship pose detection system with the SPA to predict landing Go states. This objective was further expanded, with the completion of the first contribution of this research; the development of an AHC system that uses the SPA to predict future heave motion and preemptively adjust UAV position to reduce the possibility of ship-UAV interceptions. The AHC system was also used to plan trajectories to land the UAV with a specified impact velocity. An initiative was also taken to develop a system to improve landing performance that does not use signal prediction. The second contribution of this thesis was the development of the LPI system. The LPI system was found to be an improvement over the unregulated landing system, however, the LPI was most effective when combined with the AHC system. A state-machine flight controller was introduced to the model to autonomously land the UAV based on Go signals from the SPA and LPI. The flight controller used three different landing modes:

- In Mode 1, the flight controller landed the UAV from a $2.5 \mathrm{~m}$ low hover position with a $0.5 \mathrm{~m} / \mathrm{s}$ velocity

- In Mode 2, the flight controller landed the UAV from a $5 \mathrm{~m}$ low hover with a linear piece-wise velocity

- In Mode 3, the flight controller landed the UAV based on trajectories formulated by the AHC from either a $2.5 \mathrm{~m}$ or $5 \mathrm{~m}$ low hover

The third objective was to analyze the effectiveness of each system and highlight 
their operating bounds. It was found that the unregulated system is proficient for landing up to sea state 3 . The LPI was found to safely land the UAV in up to sea state 4. However, when the LPI is combined with the AHC there was up to $76 \%$ success rate for landing in sea state 6 . As a standalone system the SPA is effective for safely landing up to sea state 4, and the performance was improved when combined with the AHC. The overall best landing system was found to be when the LPI, SPA and $\mathrm{AHC}$ were combined, having up to a $90 \%$ success rate for all the simulations with potential to reach $98 \%$ with further tuning of the SPA.

\subsection{Future Work}

For future work, a higher fidelity model could be implemented by introducing and correcting for Inertial Measurement Unit (IMU) drift and noise. The current model assumes the UAV has exact information about its orientation, which is rarely the case. Typically, sensor fusion between GPS data, IMU measurements, magnetic field readings, and visual feedback are used to estimate UAV orientation [87-90]. Future models may also benefit from a CFD analysis of the UAV during various stages of flight to better capture induced drag, propeller vortices, and superstructure air wake. Further, a vibrational sensitivity analysis could be performed to determine the feasibility of using single entity LIDARs on a UAV in close proximity to the motors.

In this thesis aircraft control was found to be satisfactory, however, more robust control techniques could be investigated to further improve the trajectory tracking performance. More robust control may even be necessary with the implementation of superstructure air wake turbulence. Future models could also benefit from UAV-deck interaction modelling, such as, sliding and landing gear impact force analysis. FEM modelling may be useful for modelling UAV-deck interactions, however, standard dynamic analysis could also be used. 
In the current model, four single entity LIDARs were coupled with the UAV's IMU data to determine ship deck displacements. Despite the satisfactory results found in this thesis, it is hypothesized that landing performance would be improved by having more accurate SPA predictions, which may be achieved if LIDAR noise is reduced. It is suggested that other pose estimation techniques, such as using a flash LIDAR should be investigated. Future work may also benefit from sensor fusion between LIDAR, sonar, and visual mapping techniques. It is also recommended that landing zone tracking techniques independent of ship communication should be investigated to further improve the cross-platform capability between UAVs and ally ships.

However, before future models are developed it would be useful to first validate aspects of the current model, such as the method used for ship pose detection and LIDAR filtering. Further, it is important to validate the effectiveness of the SPA for real ship motion as the simulated ship motion can be considered deterministic compared to physical ship motion which is stochastic by nature. Overall, the results in this thesis are a demonstration of the potential improvement the SPA can provide to rotorcraft-ship landings, but until further validation, remains a proof-of-concept. 


\section{Bibliography}

[1] S.O. Young et al. A maritime surveillance project using small uav. 2007 IEEE Conference on Emerging Technologies and Factory Automation, pages 904-907, 2007.

[2] M. Faria et al. Coordinating uavs and auvs for oceanographic field experiments: Challenges and lessons learned. 2014 IEEE International Conference on Robotics and Automation (ICRA), pages 6606-6611, 2014.

[3] J. Fortuna et al. Using low cost open source uavs for marine wild life monitoringfield report. IFAC Proceedings Volumes, 46(30):291-295, 2013.

[4] P. Westall et al. Vision-based uav maritime search and rescue using point target detection. In Proceedings of Twelfth Australian International Aerospace Congress, 2007.

[5] United States Coast Guard. Shipboard-helicopter operational procedures manual. pages 464-490, 2001.

[6] S. Arora et al. Infrastructure-free shipdeck tracking for autonomous landing. 2013 IEEE International Conference on Robotics and Automation, 2013.

[7] J.K Woodacre et al. Hydraulic valve-based active-heave compensation using a model-predictive controller with non-linear valve compensations. Ocean Engineering, 152:47-56, 2018. 
[8] J. McPhee et al. On-line determination of a go-nogo state using a continous estimation of the system response. In Proceedings of the Canadian Society of Mechanical Engineering International Congress 2018, 2018.

[9] S. Abujoub et al. Unmanned aerial vehicle landing on maritime vessels using signal prediction of the ship motion. In OCEANS 2018 MTS/IEEE Charleston, pages $1-9,2018$.

[10] M. Markowitz. German helicopter development 1932 to 1945 . https: //defensemedianetwork.com/series/nazi_rotors_german_helicopter_ development_1932-1945/, 2014. [Accessed: 10-May-2019].

[11] K.M. Rosen. A prospective: The importance of propulsion technology to the development of helicopter systems with a vision for the future. Journal of the American Helicopter Society, 53(4):307-337, 2008.

[12] R.G. Fox. The history of helicopter safety. International Helicopter Safety Symposium, pages 1-17, 2005.

[13] FAA. 2001-2014 helicopter forecasts. (8):VI.1-VI.8, 2002.

[14] Gov.Canada. Statistical summary: Air transportation occurrences in 2017. Technical report, 2018.

[15] C.R Aragon. Airflow hazard visualization for helicopter pilots: Flight simulation study results. Annual Forum Proceedings-American Helicopter Society, 61(1):1, 2005.

[16] FAS. Flight deck and operations. https://fas.org/man/dod-101/navy/docs/ swos/deck/STU15 2.html, 2015. [Accessed: April-2019].

[17] Curtiss-Wright. Curtiss-wright-naval-handling-systems. 
[18] Heligrid. Heligrid anchoring landings platform stanag 1276.

[19] M.T Silva. Ocean surface wave spectrum. Technical report, 2015.

[20] W.J. Pierson et al. A proposed spectral form for fully developed wind seas based on the similarity theory of sa kitaigorodskii. Journal of Geophysical Research, 69(24):5181-5190, 1964 .

[21] T.Pérez et al. Simulation of ship motion in seaway. Technical report, 2002.

[22] DSA-Ltd. Shipmo3d. https://dsa-ltd.ca/shipmo3d/overview/. [Accessed: Dec-2018].

[23] K. McTaggart. Validation of shipmo3d version 1.0 user applications for simulation of ship motion. Technical report, Defence Research and Development Canada, 2007.

[24] WMO. Oceanic conditions. https://wmo.int/pages/prog/amp/mmop/faq. html, 2018. [Accessed: 15-May-2019].

[25] WES. Douglas sea scale. https://encyclopedia.com/science/ encyclopedias-almanacs-transcripts-and-maps/douglas-sea-scale, 2003. [Accessed: 15-May-2019].

[26] H.J. Isemer et al. The scientific beaufort equivalent scale: Effects on wind statistics and climatological air-sea flux estimates in the north atlantic ocean. Journal of Climate, 4(8):819-836, 1991.

[27] J.L Sanchez-Lopez et al. Toward visual autonomous ship board landing of a vtol uav. 2013 International Conference on Unmanned Aircraft Systems, pages 779-788, 2013. 
[28] S.R. Oh et al. Autonomous helicopter landing on a moving platform using a tether. Proceedings of the 2005 IEEE International Conference on Robotics and Automation, pages 3960-3965, 2005.

[29] W.Kong et al. Autonomous landing of an uav with a ground-based actuated infrared stereo vision system. 2013 IEEE/RSJ International Conference on Intelligent Robots and Systems, pages 2963-2970, 2013.

[30] M. Garratt et al. Non-linear control of heave for an unmanned helicopter using a neural network. Journal of Intelligent $\mathcal{E}$ Robotic Systems, 66(4):495-504, 2012.

[31] P. Moriarty et al. Neural networks to aid the autonomous landing of a uav on a ship. 2017 28th Irish Signals and Systems Conference, pages 1-4, 2017.

[32] J.R. Hervas et al. Automatic landing control of unmanned aerial vehicles on moving platforms. 2014 IEEE 23rd international symposium on industrial electronics, pages 69-74, 2014.

[33] T.D. Ngo et al. Nonlinear helicopter and ship models for predictive control of ship landing operations. AIAA Guidance, Navigation, and Control Conference, page 1298, 2014.

[34] C.K. Fourie. The autonomous landing of an unmanned helicopter on a moving platform. PhD thesis, 2015.

[35] B. Ferrier et al. Simulation and testing of the landing period designator helicopter recovery aid. Naval Engineers Journal, 110(1):189-205, 1998.

[36] G.G. Huang et al. Short-term prediction of ship pitching motion based on artificial neural networks. ASME 2016 35th International Conference on Ocean, Offshore and Arctic Engineering, 2016. 
[37] X.Y. PENG et al. Research on real-time prediction algorithm of ship attitude motion. Journal of System Simulation, 2, 2007.

[38] Y.M. Chen et al. Experiment of extremely short-term prediction of ship motion. Ship $\&$ Ocean Engineering, 39(1):13-15, 2010.

[39] X. Zhao et al. Ship-motion prediction: algorithms and simulation results. 2004 IEEE International Conference on Acoustics, Speech, and Signal Processing, 5:120-125, 2004.

[40] J.C. Chung et al. A note on ship-motion prediction based on wave-excitation input estimation. IEEE Journal of Oceanic Engineering, 15(3):244-250, 1990.

[41] J.Li et al. Dynamic analysis and pid control for a quadrotor. 2011 IEEE International Conference on Mechatronics and Automation, pages 573-578, 2011.

[42] D. Gautam et al. Control of a quadrotor using a smart self-tuning fuzzy pid controller. International Journal of Advanced Robotic Systems, 10(11):380, 2013.

[43] S. Bouabdallah et al. Pid vs lq control techniques applied to an indoor micro quadrotor. 2004 IEEE International Conference on Intelligent Robots and Systems, 3:2451-2456, 2004.

[44] A.L Salih et al. Flight pid controller design for a uav quadrotor. Scientific Research and Essays, 5(23):3660-3667, 2010.

[45] Pixhawk. Multicopter pid tuning guide px4 v1.9.0. https://docs.px4.io/v1. 9.0/en/config_mc/pid_tuning_guide_multicopter.html. [Accessed: Oct2019].

[46] Ardupilot. Advanced tuning copter documentation. http://ardupilot.org/ copter/docs/tuning.html, 2019. [accessed: Oct-2019]. 
[47] P. Wang et al. Dynamics modelling and linear control of quadcopter. 2016 International Conference on Advanced Mechatronic Systems (ICAMechS), pages 498-503, 2016.

[48] J.D Anderson et al. Computational fluid dynamics, volume 206. Springer, 1995.

[49] W. Zuo. Introduction of computational fluid dynamics. FAU Erlange-Nümberg, 2005.

[50] K.Hughes et al. Application of the finite element method to predict the crashworthy response of a metallic helicopter under floor structure onto water. International journal of impact engineering, 35(5):347-362, 2008.

[51] O.A. Bauchau et al. Modeling rotorcraft dynamics with finite element multibody procedures. In Elsevier Mathematical and Computer Modelling, 33(10-11):11131137, 2001.

[52] M.C Bogstad et al. Computational-fluid-dynamics based advanced ship-airwake database for helicopter flight simulators. Journal of Aircraft, 39(5):830-838, 2002.

[53] R.Steijl et al. A framework for cfd analysis of helicopter rotors in hover and forward flight. International journal for Numerical Methods in Fluids, 51(8):819$847,2006$.

[54] T.Takami et al. A numerical simulation method for predicting global and local hydroelastic response of a ship based on cfd and fea coupling. Marine Structures, 59:368-386, 2018.

[55] M.Aoki. State space modeling of time series. Springer Science, 2013.

[56] R.Pintelon et al. System identification: a frequency domain approach. John Wiley \& Sons, 2012. 
[57] L.R. Ribeiro. Uav autopilot controllers test platform using matlab/simulink and x-plane. 2010 IEEE Frontiers in Education Conference, 2010.

[58] L.D. Minh et al. Modeling and control of quadrotor mav using vision-based measurement. International Forum on Strategic Technology 2010, pages 70-75, 2010.

[59] DJI. Dji-specification sheet-phantom. https://dji.com/ca/phantom-3-pro/ info, 2019. [Accessed: Sept-2019].

[60] DJI. Dji-specification sheet, martice-200 series. https://dji.com/ca/ matrice-200-series/info, 2019. [Accessed: Sept-2019].

[61] FlyBrushless.com. Rcn 600/20 motor specification data sheet. http:// flybrushless.com/motor/view/191. [Accessed: Sept-2019].

[62] E.M. Greitzer et al. Thermodynamics and propulsion. 2011. MIT OpenCourseWare.

[63] G. Staples. Propeller static and dynamic thrust calculation. https://electricrcaircraftguy.com/2014/04/ propeller-static-dynamic-thrust-equation-background.html, 2014. Accessed: 3-Oct-2019.

[64] R.W. Deters et al. Reynolds number effects on the performance of small-scale propellers. 32nd Applied Aerodynamics Conference, 2014.

[65] J. Brandt et al. Propeller performance data at low reynolds numbers. 49th AIAA Aerospace Sciences Meeting, page 1255, 2011.

[66] MIT. Dc motor \& propeller matching. http://web.mit.edu/drela/Public/ web/qprop/motorprop.pdf, March 2005. [Accessed: March-2019]. 
[67] A. Betz. The ground effect on lifting propellers. Technical report, 1937.

[68] I.C Cheeseman et al. The effect of ground on a helicopter rotor in forward flight. 1955.

[69] X. Ortiz et al. Forces and moments on flat plates of small aspect ratio with application to pv wind loads and small wind turbine blades. Energies, 8(4):24382453,2015 .

[70] Engineer Tool Box. Drag coefficient, 2004.

[71] R.S. Shevell. Fundamentals of Flight. Prentice Hall, 1989.

[72] A.H. Techet. Ocean waves. ocw.mit.edu/courses/mechanical-engineering/ 2-22-design-principles-for-ocean-vehicles-13-42-spring-2005/ readings/lec6_wavespectra.pdf, 2005. [Accessed: 7-May-2019].

[73] K. Hasselmann et al. Measurements of wind-wave growth and swell decay during the joint north sea wave project. Ergänzungsheft 8-12, 1973.

[74] U.S. Military. Military specification mil-f-8785c. 1980.

[75] MathWorks. Dryden wind turbulence model (continuous). https://mathworks . com/help/aeroblks/drydenwindturbulencemodelcontinuous.html, 2006. [Accessed: April-2019].

[76] G.V. Raffo et al. An integral predictive/nonlinear h control structure for a quadrotor helicopter. Automatica, 46(1):29-39, 2010.

[77] A. Mian et al. Modeling and backstepping-based nonlinear control strategy for a 6 dof quadrotor helicopter. Chinese Journal of Aeronautics, 21(3):261-268, 2008 . 
[78] Y.M. Al-Younes et al. Linear vs. nonlinear control techniques for a quadrotor vehicle. Yth International Symposium on Mechatronics and its Applications, pages $1-10,2010$.

[79] J. Junior et al. Stability control of a quad-rotor using a pid controller. Brazilian Journal of Instrumentation and Control, 1(1):15-20, 2013.

[80] S.Khatoon et al. Dynamic modeling and stabilization of quadrotor using pid controller. 2014 International Conference on Advances in Computing, Communications and Informatics (ICACCI), pages 746-750, 2014.

[81] J. Zhong. Pid controller tuning: A short tutorial. Mechanical Engineering, Purdue University, pages 1-10, 2006.

[82] Garmin. Lidar lite operation manual and technical specifications, 2016.

[83] P. Church. Lidar architecture for harsh environment applications. EPJ Web of Conferences, 119, 2016.

[84] S.Küchler et al. Active control for an offshore crane using prediction of the vessels motion. IEEE/ASME Transactions on Mechatronics, 16(2):297-309, 2010.

[85] Jeff Dodge. Mq-8 and unmanned launch and recovery, 2016.

[86] B. Ferrier et al. Fire scout launch and recovery considerations in unexpected ship roll motion conditions. American Society of Naval Engineers Journal, 129(4):87$98,2017$.

[87] A.Z Azfar et al. A simple approach on implementing imu sensor fusion in pid controller for stabilizing quadrotor flight control. 2011 IEEE 7th International Colloquium on Signal Processing and its Applications, pages 28-32, 2011. 
[88] M. Tailanián et al. Design and implementation of sensor data fusion for an autonomous quadrotor. 2014 IEEE International Instrumentation and Measurement Technology Conference Proceedings, pages 1431-1436, 2014.

[89] S. Wang et al. Quadrotor aircraft attitude estimation and control based on kalman filter. Proceedings of the 31st Chinese Control Conference, pages 5634$5639,2012$.

[90] L.Kis et al. Sensor fusion and actuator system of a quadrotor helicopter. Periodica Polytechnica Electrical Engineering, 53(3-4):139-150, 2011. 
Appendix A

Full Results - Low Hover, $2.5 \mathrm{~m}$ 
Table A.1: Unregulated 2.5m low hover full results.

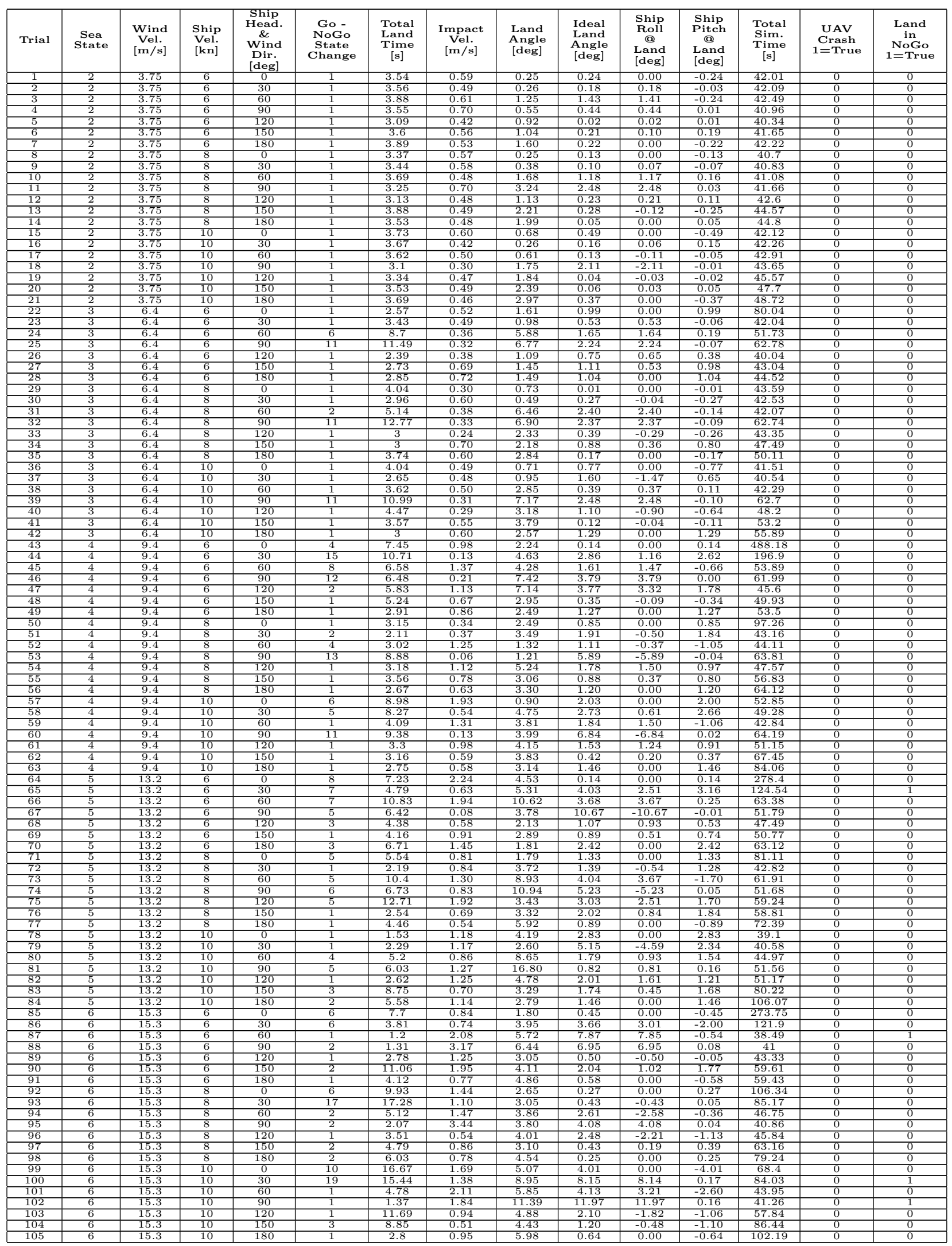


Table A.2: SPA 2.5m low hover full results.

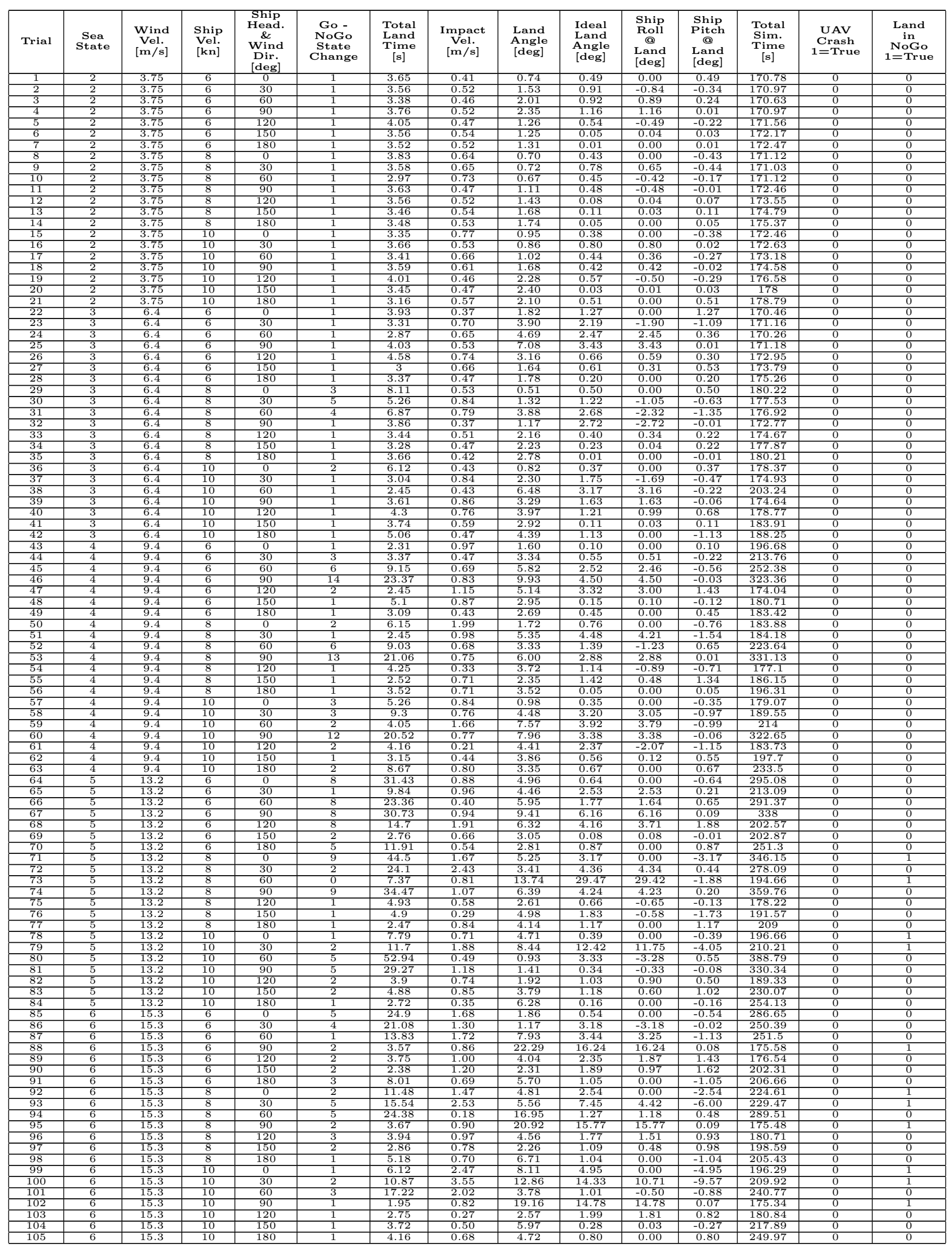


Table A.3: SPA+AHC 2.5m low hover full results.

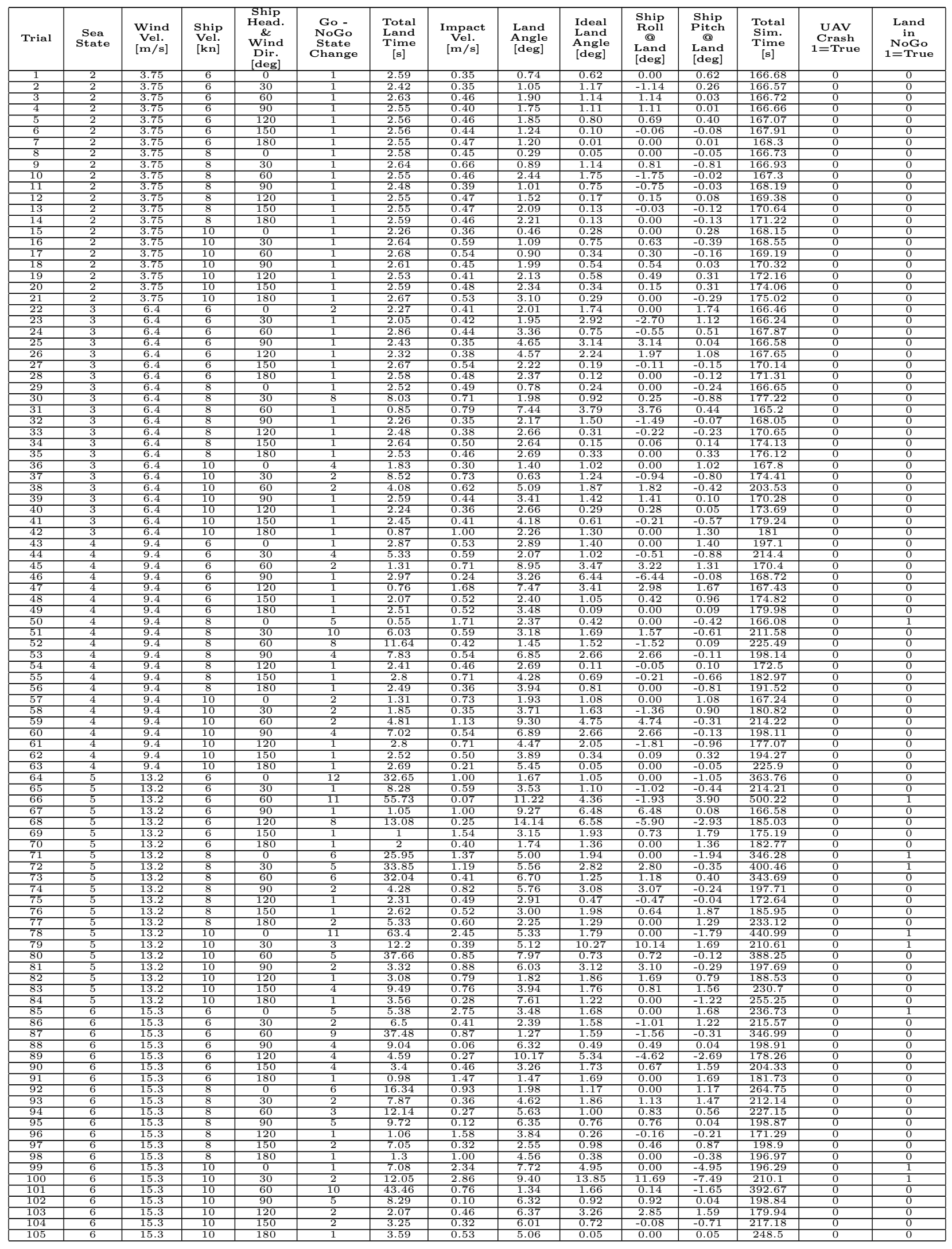


Table A.4: LPI 2.5m low hover full results.

\begin{tabular}{|c|c|c|c|c|c|c|c|c|c|c|c|c|c|c|}
\hline Trial & $\begin{array}{l}\text { Sea } \\
\text { State }\end{array}$ & $\begin{array}{l}\text { Wind } \\
\text { Vel. } \\
{[\mathrm{m} / \mathrm{s}]}\end{array}$ & $\begin{array}{l}\text { Ship } \\
\text { Vel } \\
\text { [kn] }\end{array}$ & $\begin{array}{l}\text { Ship } \\
\text { Head. } \\
\& \text { \& } \\
\text { wind } \\
\text { Dir. } \\
\text { [deg] }\end{array}$ & $\begin{array}{c}\text { Go- } \\
\text { NoGo } \\
\text { State } \\
\text { Change }\end{array}$ & $\begin{array}{c}\text { Total } \\
\text { Land } \\
\text { Time } \\
{[\mathrm{s}]}\end{array}$ & $\begin{array}{c}\text { Impact } \\
\text { Vel. } \\
{[\mathbf{m} / \mathrm{s}]}\end{array}$ & $\begin{array}{c}\text { Land } \\
\text { Angle } \\
\text { [deg] }\end{array}$ & $\begin{array}{l}\text { Ideal } \\
\text { Land } \\
\text { Angle } \\
\text { [deg] }\end{array}$ & $\begin{array}{l}\text { Ship } \\
\text { Roll } \\
\text { @ } \\
\text { Land } \\
\text { [deg] }\end{array}$ & $\begin{array}{l}\text { Ship } \\
\text { Pitch } \\
\text { @ } \\
\text { Land } \\
\text { [deg] }\end{array}$ & $\begin{array}{l}\text { Total } \\
\text { Sim. } \\
\text { Time } \\
{[\text { [s] }}\end{array}$ & $\begin{array}{c}\text { UAV } \\
\text { Crash } \\
1=\text { True }\end{array}$ & $\begin{array}{c}\text { Land } \\
\text { in } \\
\text { NoGo } \\
1=\text { True }\end{array}$ \\
\hline$\frac{1}{2}$ & $\frac{2}{2}$ & $\begin{array}{l}3.75 \\
3.75\end{array}$ & $\frac{6}{6}$ & $\begin{array}{c}0 \\
0 \\
30\end{array}$ & $\frac{1}{1}$ & $\begin{array}{l}3.34 \\
3.74 \\
\end{array}$ & $\begin{array}{l}0.45 \\
0.59\end{array}$ & $\begin{array}{l}0.22 \\
1.46\end{array}$ & $\begin{array}{l}0.11 \\
0.93\end{array}$ & $\begin{array}{l}0.00 \\
0.93 \\
\end{array}$ & $\begin{array}{c}-0.11 \\
-0.01\end{array}$ & $\begin{array}{l}160.81 \\
160.77\end{array}$ & 0 & 0 \\
\hline 3 & 2 & 3.75 & 6 & 60 & & 3.06 & 0.65 & 0.43 & 1.26 & -1.26 & 0.00 & 160.63 & 0 & 0 \\
\hline \begin{tabular}{|l}
4 \\
5
\end{tabular} & $\frac{2}{2}$ & $\begin{array}{l}3.75 \\
3.75\end{array}$ & $\frac{6}{6}$ & $\begin{array}{l}90 \\
120 \\
\end{array}$ & $\frac{1}{1}$ & $\begin{array}{l}3.15 \\
3.5\end{array}$ & 0.40 & $\begin{array}{l}0.79 \\
120\end{array}$ & $\begin{array}{l}1.11 \\
0.38\end{array}$ & \begin{tabular}{|l|}
-1.11 \\
0.33
\end{tabular} & $\begin{array}{c}-0.02 \\
0.18\end{array}$ & 160.56 & 0 & 0 \\
\hline & $\frac{2}{2}$ & $\frac{3.75}{3.75}$ & 6 & $\frac{120}{150}$ & $\frac{1}{1}$ & $\begin{array}{l}3.5 \\
3.45\end{array}$ & $\frac{0.54}{0.53}$ & $\frac{1.20}{0.99}$ & 0.38 & $\begin{array}{l}0.33 \\
0.09\end{array}$ & $\frac{0.18}{0.20}$ & $\frac{161.21}{162.06}$ & $\frac{0}{0}$ & $\frac{0}{0}$ \\
\hline 7 & 2 & 3.75 & 6 & 180 & 1 & $\begin{array}{l}0.40 \\
3.57 \\
\end{array}$ & 0.50 & 1.26 & $\begin{array}{l}0.21 \\
0.01 \\
\end{array}$ & $\begin{array}{l}0.09 \\
0.00\end{array}$ & $\begin{array}{c}0.20 \\
-0.01 \\
\end{array}$ & $\frac{162.06}{162.5}$ & 0 & 0 \\
\hline $\begin{array}{l}8 \\
9\end{array}$ & $\frac{2}{2}$ & $\begin{array}{l}3.75 \\
3.75 \\
\end{array}$ & 8 & $\begin{array}{l}0 \\
30\end{array}$ & 1 & $\begin{array}{l}3.62 \\
365\end{array}$ & $\begin{array}{l}0.63 \\
0.48\end{array}$ & $\begin{array}{l}0.77 \\
0.66\end{array}$ & $\begin{array}{l}0.32 \\
0.74\end{array}$ & $\begin{array}{l}0.00 \\
-0.74\end{array}$ & $\begin{array}{l}-0.32 \\
0.05\end{array}$ & 160.77 & 0 & 0 \\
\hline $\begin{array}{l}9 \\
10\end{array}$ & $\frac{2}{2}$ & $\begin{array}{l}3.75 \\
3.75\end{array}$ & $\frac{8}{8}$ & $\frac{30}{60}$ & $\frac{1}{1}$ & $\begin{array}{l}3.65 \\
3.45\end{array}$ & $\frac{0.48}{0.56}$ & $\begin{array}{l}0.66 \\
1.64\end{array}$ & $\begin{array}{l}0.74 \\
0.32\end{array}$ & $\frac{-0.74}{0.26}$ & $\begin{array}{l}0.05 \\
0.18\end{array}$ & $\frac{161.34}{161.44}$ & $\frac{0}{0}$ & $\frac{0}{0}$ \\
\hline 11 & & $\begin{array}{l}.75 \\
3.75 \\
\end{array}$ & 8 & 90 & & 3.67 & 0.48 & 1.09 & 0.00 & 0.00 & 0.00 & 162.3 & 0 & 0 \\
\hline$\frac{12}{13}$ & $\frac{2}{2}$ & $\begin{array}{r}3.75 \\
3.75 \\
\end{array}$ & $\frac{8}{8}$ & 120 & 1 & $\begin{array}{l}3.39 \\
3.48\end{array}$ & 0.46 & $\begin{array}{l}1.52 \\
1.56\end{array}$ & $\begin{array}{l}0.07 \\
0.06\end{array}$ & $\frac{-0.07}{0.01}$ & $\frac{-0.02}{0.06}$ & 163.34 & 0 & 0 \\
\hline 13 & $\frac{2}{2}$ & $\begin{array}{l}3.15 \\
3.75 \\
\end{array}$ & 8 & 180 & $\frac{1}{1}$ & $\begin{array}{l}3.48 \\
3.48 \\
\end{array}$ & $\frac{0.53}{0.51}$ & $\begin{array}{l}1.56 \\
1.54 \\
\end{array}$ & $\begin{array}{l}0.06 \\
0.09\end{array}$ & $\begin{array}{l}0.01 \\
0.00\end{array}$ & $\begin{array}{l}0.06 \\
0.09\end{array}$ & $\begin{array}{l}164.79 \\
165.35\end{array}$ & $\frac{0}{0}$ & $\frac{0}{0}$ \\
\hline 15 & $\frac{2}{2}$ & $\begin{array}{l}3.75 \\
\end{array}$ & 10 & 0 & & $\begin{array}{l}3.41 \\
361\end{array}$ & 0.60 & $\begin{array}{l}0.28 \\
0.76\end{array}$ & 0.23 & 0.00 & $\begin{array}{l}-0.23 \\
-0.34\end{array}$ & $\begin{array}{l}162.64 \\
62.68\end{array}$ & 0 & 0 \\
\hline $\begin{array}{l}16 \\
17\end{array}$ & $\frac{2}{2}$ & $\begin{array}{r}3.75 \\
3.75\end{array}$ & $\frac{10}{10}$ & $\begin{array}{l}30 \\
60\end{array}$ & $\frac{1}{1}$ & \begin{tabular}{|l|l|}
3.61 \\
4.27
\end{tabular} & $\begin{array}{l}0.68 \\
0.36\end{array}$ & $\begin{array}{l}0.76 \\
0.45\end{array}$ & $\begin{array}{l}0.45 \\
1.09\end{array}$ & $\begin{array}{l}-0.30 \\
-1.09\end{array}$ & $\begin{array}{c}-0.34 \\
-0.04\end{array}$ & $\frac{162.68}{163.58}$ & $\frac{0}{0}$ & $\frac{0}{0}$ \\
\hline 18 & 2 & 3.75 & 10 & 90 & 1 & 3.79 & 0.56 & 1.44 & $\begin{array}{l}1.05 \\
0.60\end{array}$ & $\frac{-1.09}{-0.60}$ & $\frac{-0.04}{-0.01}$ & $\begin{array}{l}103.08 \\
164.74\end{array}$ & 0 & 0 \\
\hline $\begin{array}{l}19 \\
20\end{array}$ & $\frac{2}{2}$ & $\begin{array}{l}3.75 \\
3.75\end{array}$ & $\frac{10}{10}$ & $\begin{array}{l}120 \\
150\end{array}$ & 1 & $\begin{array}{l}3.55 \\
3.77\end{array}$ & 0.43 & $\frac{1.82}{2.699}$ & 0.07 & $\begin{array}{l}-0.04 \\
-0.16\end{array}$ & $\begin{array}{l}-0.06 \\
\end{array}$ & 166.2 & 0 & 0 \\
\hline$\frac{20}{21}$ & $\frac{2}{2}$ & $\begin{array}{l}3.75 \\
3.75\end{array}$ & $\begin{array}{l}\frac{10}{10} \\
10\end{array}$ & $\begin{array}{l}\frac{150}{180} \\
\end{array}$ & $\frac{1}{1}$ & \begin{tabular}{|l|l|}
3.77 \\
3.49
\end{tabular} & $\begin{array}{l}0.43 \\
0.45\end{array}$ & $\begin{array}{l}2.69 \\
2.48\end{array}$ & $\begin{array}{l}0.42 \\
0.06\end{array}$ & $\frac{-0.16}{0.00}$ & $\begin{array}{c}-0.39 \\
0.06\end{array}$ & $\frac{168.24}{168.84}$ & $\frac{0}{0}$ & $\frac{0}{0}$ \\
\hline 22 & 3 & $\begin{array}{r}0.4 \\
\end{array}$ & 6 & 0 & 1 & $\begin{array}{l}0.49 \\
2.79 \\
\end{array}$ & 0.28 & $\begin{array}{l}2.48 \\
1.12 \\
\end{array}$ & $\begin{array}{l}0.06 \\
0.11 \\
\end{array}$ & $\begin{array}{l}0.00 \\
0.00 \\
\end{array}$ & $\begin{array}{r}0.06 \\
-0.11 \\
\end{array}$ & $\begin{array}{l}100.046 \\
\end{array}$ & 0 & 0 \\
\hline$\frac{23}{24}$ & $\begin{array}{l}3 \\
3 \\
\end{array}$ & \begin{tabular}{|l|}
6.4 \\
6.4
\end{tabular} & $\frac{6}{6}$ & $\begin{array}{l}30 \\
60\end{array}$ & 1 & \begin{tabular}{|l|l|}
3.34 \\
3.08
\end{tabular} & $\frac{0.72}{0.26}$ & $\begin{array}{l}3.66 \\
5.32 \\
\end{array}$ & \begin{tabular}{|l|}
2.73 \\
1.07
\end{tabular} & $\begin{array}{l}2.73 \\
0.84\end{array}$ & $\begin{array}{l}0.02 \\
0.67\end{array}$ & $\begin{array}{l}164.57 \\
16767\end{array}$ & 0 & 0 \\
\hline 25 & $\frac{3}{3}$ & $\begin{array}{l}0.4 \\
6.4\end{array}$ & 6 & 90 & $\frac{1}{1}$ & $\frac{3.08}{2.07}$ & $\frac{0.26}{0.50}$ & $\begin{array}{l}5.32 \\
5.05 \\
\end{array}$ & $\frac{1.07}{2.07}$ & $\frac{0.84}{2.07}$ & 0.05 & $\frac{167.61}{159.64}$ & $\frac{0}{0}$ & 0 \\
\hline $\begin{array}{l}26 \\
27\end{array}$ & 3 & $\begin{array}{l}6.4 \\
6.4\end{array}$ & 6 & 120 & 1 & 3.21 & 0.52 & 2.67 & 1.09 & 0.96 & 0.52 & 161.76 & 0 & 0 \\
\hline$\frac{27}{28}$ & $\frac{3}{3}$ & $\begin{array}{l}6.4 \\
6.4\end{array}$ & $\frac{6}{6}$ & $\frac{150}{180}$ & 1 & $\begin{array}{l}3.09 \\
3.46\end{array}$ & $\frac{0.51}{0.52}$ & $\begin{array}{l}1.42 \\
1.57 \\
1.57\end{array}$ & $\begin{array}{l}0.40 \\
0.02\end{array}$ & $\begin{array}{l}0.19 \\
0.00\end{array}$ & $\begin{array}{l}0.35 \\
0.02\end{array}$ & $\begin{array}{l}163.78 \\
165.41\end{array}$ & $\frac{0}{0}$ & 0 \\
\hline 28 & $\frac{3}{3}$ & $\begin{array}{l}6.4 \\
6.4\end{array}$ & $\frac{6}{8}$ & $\frac{180}{0}$ & $\frac{1}{1}$ & $\begin{array}{l}3.46 \\
4.32\end{array}$ & $\frac{0.52}{0.90}$ & $\frac{1.57}{2.02}$ & $\begin{array}{l}0.02 \\
1.27\end{array}$ & $\begin{array}{l}0.00 \\
0.00\end{array}$ & $\begin{array}{r}0.02 \\
-1.28\end{array}$ & $\frac{165.41}{165.43}$ & $\frac{0}{0}$ & $\frac{0}{0}$ \\
\hline 30 & 3 & 6.4 & 8 & 30 & 1 & $\begin{array}{l}2.84 \\
3.7\end{array}$ & 0.81 & $\begin{array}{l}3.79 \\
\end{array}$ & 3.49 & 3.11 & -1.58 & 163.23 & 0 & 0 \\
\hline$\frac{31}{32}$ & 3 & $\begin{array}{l}6.4 \\
\end{array}$ & 8 & 60 & 1 & $\begin{array}{l}3.7 \\
3.82\end{array}$ & 0.90 & $\begin{array}{l}4.75 \\
127\end{array}$ & 1.57 & 1.56 & -0.19 & 161.09 & 0 & 0 \\
\hline 32 & 3 & $\begin{array}{r}6.4 \\
6.4\end{array}$ & 8 & $\frac{90}{120}$ & $\frac{1}{1}$ & $\begin{array}{l}3.82 \\
3.75\end{array}$ & $\frac{0.43}{0.34}$ & $\frac{1.27}{2.84}$ & $\begin{array}{l}0.16 \\
0.94\end{array}$ & $\frac{-0.16}{-0.77}$ & $\frac{-0.01}{-0.55}$ & $\frac{162.27}{164.7}$ & 0 & $\begin{array}{l}0 \\
0 \\
0\end{array}$ \\
\hline 34 & 3 & 6.4 & 8 & 150 & 1 & $\begin{array}{l}0.10 \\
3.39\end{array}$ & 0.47 & $\frac{2.04}{2.20}$ & 0.12 & $\frac{-0.11}{0.01}$ & $\frac{-0.00}{0.12}$ & 168 & 0 & 0 \\
\hline 35 & 3 & 6.4 & 8 & 180 & 1 & 3.8 & 0.44 & 3.09 & 0.23 & 0.00 & -0.23 & 170.43 & 0 & 0 \\
\hline 36 & 3 & $\begin{array}{l}6.4 \\
6\end{array}$ & 10 & 0 & $\frac{1}{2}$ & 2.93 & 0.19 & 1.83 & 2.06 & 0.00 & 2.00 & 163.96 & 0 & 0 \\
\hline $\begin{array}{l}37 \\
38\end{array}$ & 3 & $\begin{array}{l}6.4 \\
6.4\end{array}$ & $\frac{10}{10}$ & $\frac{30}{60}$ & $\frac{2}{4}$ & $\begin{array}{l}7.94 \\
6.88\end{array}$ & 1.08 & $\begin{array}{l}2.46 \\
3.48\end{array}$ & $\begin{array}{l}2.25 \\
3.24\end{array}$ & $\begin{array}{c}0.53 \\
-3.12\end{array}$ & $\begin{array}{r}-2.19 \\
-0.87\end{array}$ & $\begin{array}{l}178.33 \\
200.87\end{array}$ & 0 & 0 \\
\hline 39 & & 6.4 & 10 & 90 & 1 & 4.33 & 0.75 & 1.94 & 1.88 & -1.88 & -0.03 & 165.06 & 0 & \\
\hline 40 & 3 & 6.4 & 10 & 120 & 1 & 4.59 & 0.49 & 3.18 & 0.93 & -0.80 & -0.48 & 168.9 & 0 & 0 \\
\hline 41 & 3 & $\begin{array}{l}6.4 \\
6.4\end{array}$ & 10 & 150 & $T$ & $\begin{array}{l}3.23 \\
3009\end{array}$ & 0.64 & $\begin{array}{l}2.99 \\
323\end{array}$ & 0.81 & 0.36 & 0.72 & $\begin{array}{l}173.62 \\
\end{array}$ & 0 & 0 \\
\hline $\begin{array}{l}42 \\
43 \\
\end{array}$ & $\begin{array}{l}3 \\
4 \\
\end{array}$ & $\begin{array}{l}6.4 \\
9.4\end{array}$ & $\frac{10}{6}$ & $\frac{180}{0}$ & $\frac{1}{3}$ & \begin{tabular}{|l|l|}
3.09 \\
4.43
\end{tabular} & 0.70 & \begin{tabular}{|l|l|}
3.23 \\
3.22 \\
\end{tabular} & $\begin{array}{l}0.56 \\
1.70\end{array}$ & $\begin{array}{l}0.00 \\
0.00\end{array}$ & $\begin{array}{l}0.56 \\
1.70 \\
1.0\end{array}$ & $\begin{array}{l}176.94 \\
1972\end{array}$ & 0 & 0 \\
\hline$\frac{45}{44}$ & $\frac{4}{4}$ & $\begin{array}{l}5.4 \\
9.4\end{array}$ & $\frac{0}{6}$ & 30 & $\frac{3}{1}$ & $\begin{array}{l}.458 \\
2.98 \\
\end{array}$ & $\frac{1.11}{1.00}$ & $\begin{array}{l}3.22 \\
4.18 \\
\end{array}$ & 0.38 & 0.00 & $\frac{1.05}{0.05}$ & $\begin{array}{l}191.2 \\
202.39\end{array}$ & 0 & 0 \\
\hline 45 & 4 & $\begin{array}{l}9.4 \\
9.4\end{array}$ & & 60 & 3 & 8.71 & 0.80 & 5.97 & $\begin{array}{l}2.66 \\
2.74\end{array}$ & \begin{tabular}{|c|}
-1.80 \\
2.74
\end{tabular} & $\begin{array}{l}-1.95 \\
0.02\end{array}$ & $\begin{array}{l}176.88 \\
35.5\end{array}$ & 0 & 0 \\
\hline $\begin{array}{l}46 \\
47\end{array}$ & $\frac{4}{4}$ & $\begin{array}{l}9.4 \\
9.4\end{array}$ & $\frac{6}{6}$ & $\frac{90}{120}$ & $\frac{6}{1}$ & $\begin{array}{l}18.94 \\
3.11\end{array}$ & $\frac{0.39}{0.25}$ & $\frac{7.60}{2.28}$ & $\begin{array}{l}2.74 \\
0.32\end{array}$ & $\begin{array}{r}2.74 \\
-0.26\end{array}$ & $\begin{array}{l}0.02 \\
-0.18\end{array}$ & $\begin{array}{l}315.25 \\
16308\end{array}$ & $\frac{0}{0}$ & 0 \\
\hline 48 & 4 & 9.4 & 6 & 150 & 1 & $\begin{array}{l}0.19 \\
4.49\end{array}$ & 0.52 & 2.96 & 0.03 & $\begin{array}{r}-0.20 \\
-0.01\end{array}$ & $\begin{array}{r}-0.10 \\
0.03 \\
\end{array}$ & 170.36 & 0 & 0 \\
\hline $\begin{array}{l}49 \\
50\end{array}$ & 4 & \begin{tabular}{|l|l}
9.4 \\
9.4
\end{tabular} & $\begin{array}{l}6 \\
8\end{array}$ & 180 & $\frac{1}{6}$ & $\begin{array}{l}3.97 \\
20.97\end{array}$ & 0.49 & $\begin{array}{l}3.10 \\
148\end{array}$ & $\begin{array}{l}0.00 \\
0.39\end{array}$ & $\begin{array}{l}0.00 \\
0.00\end{array}$ & $\begin{array}{l}0.01 \\
-0.39 \\
\end{array}$ & 174.6 & 0 & 0 \\
\hline 50 & $\frac{4}{4}$ & $\begin{array}{l}9.4 \\
9.4\end{array}$ & $\frac{8}{8}$ & $\frac{0}{30}$ & $\frac{6}{4}$ & $\frac{20.97}{7.19}$ & $\begin{array}{l}0.31 \\
0.99\end{array}$ & $\begin{array}{l}1.48 \\
4.41\end{array}$ & $\begin{array}{l}0.39 \\
0.85\end{array}$ & $\begin{array}{l}0.00 \\
0.84\end{array}$ & $\begin{array}{c}-0.39 \\
0.09\end{array}$ & $\begin{array}{l}197.46 \\
184.96\end{array}$ & $\frac{0}{0}$ & $\frac{0}{0}$ \\
\hline 52 & 4 & 9.4 & 8 & 60 & $T$ & 3.65 & 1.09 & 8.79 & $\frac{0.00}{2.64}$ & $\begin{array}{l}.04 \\
2.62\end{array}$ & $\frac{0.33}{0.32}$ & 161.48 & 0 & 0 \\
\hline 54 & 4 & $\begin{array}{l}9.4 \\
9.4\end{array}$ & 8 & 90 & 4 & $\frac{10.03}{4.46}$ & 0.91 & $\begin{array}{l}4.79 \\
290\end{array}$ & $\begin{array}{l}0.70 \\
0.33\end{array}$ & $\begin{array}{l}0.69 \\
0.23\end{array}$ & 0.02 & 205.06 & 0 & 0 \\
\hline 55 & $\frac{4}{4}$ & $\begin{array}{l}9.4 \\
9.4 \\
\end{array}$ & 8 & 150 & $\frac{1}{1}$ & $\frac{4.40}{2.52}$ & 0.01 & $\frac{2.90}{2.97}$ & 0.55 & 0.40 & 0.18 & $\begin{array}{l}10.411 \\
175.99\end{array}$ & 0 & 0 \\
\hline 56 & 4 & 9.4 & 8 & 180 & t & 2.78 & 0.71 & 3.18 & 0.69 & 0.00 & 0.69 & 185.43 & 0 & 0 \\
\hline $\begin{array}{l}57 \\
58\end{array}$ & $\frac{4}{4}$ & $\begin{array}{l}9.4 \\
9.4\end{array}$ & $\frac{10}{10}$ & $\frac{0}{30}$ & $\frac{1}{4}$ & $\begin{array}{l}3.15 \\
10.5\end{array}$ & $\frac{0.93}{0.94}$ & $\begin{array}{l}1.33 \\
0.79\end{array}$ & $\begin{array}{l}1.50 \\
0.76\end{array}$ & 0.00 & $\begin{array}{l}1.50 \\
\end{array}$ & $\begin{array}{l}163.5 \\
\end{array}$ & $\frac{0}{0}$ & 0 \\
\hline 59 & & $\begin{array}{l}9.4 \\
9.4\end{array}$ & $\frac{10}{10}$ & 60 & $\frac{4}{6}$ & $\frac{10.0}{15.23}$ & $\frac{0.94}{1.71}$ & $\frac{0.19}{5.56}$ & $\begin{array}{l}0.10 \\
3.59\end{array}$ & $\frac{-0.02}{2.86}$ & $\frac{-0.00}{-2.17}$ & $\frac{192.49}{294.26}$ & 0 & 0 \\
\hline 60 & 4 & 9.4 & 10 & 90 & $\frac{2}{2}$ & 3.43 & 0.51 & 6.91 & 4.07 & 4.07 & 0.08 & 166.58 & 0 & 0 \\
\hline$\frac{61}{62}$ & $\begin{array}{l}4 \\
4\end{array}$ & $\begin{array}{l}9.4 \\
9.4\end{array}$ & $\frac{10}{10}$ & $\frac{120}{150}$ & $\frac{1}{1}$ & $\begin{array}{l}2.32 \\
4.2 \\
\end{array}$ & $\frac{1.00}{0.43}$ & $\begin{array}{l}5.94 \\
3.56 \\
\end{array}$ & 2.18 & $\begin{array}{l}1.74 \\
-0.05\end{array}$ & $\begin{array}{l}1.32 \\
-0.10\end{array}$ & $\begin{array}{l}170.91 \\
189.03\end{array}$ & 0 & 0 \\
\hline 62 & 4 & $\begin{array}{l}9.4 \\
9.4\end{array}$ & $\frac{10}{10}$ & $\frac{150}{180}$ & $\frac{1}{1}$ & $\begin{array}{l}4.2 \\
2.82\end{array}$ & $\begin{array}{l}.45 \\
0.64\end{array}$ & $\begin{array}{l}3.50 \\
5.08\end{array}$ & $\begin{array}{l}0.11 \\
0.70\end{array}$ & $\frac{-0.05}{0.00}$ & $\frac{--1.10}{0.70}$ & $\frac{189.03}{319.21}$ & 0 & $\frac{0}{0}$ \\
\hline 64 & 5 & 13.2 & 6 & 0 & 13 & 54.75 & 0.55 & 0.03 & 2.09 & 0.00 & -2.00 & 332.14 & 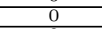 & 0 \\
\hline 65 & 5 & $\begin{array}{l}13.2 \\
32\end{array}$ & $\frac{6}{6}$ & $\frac{30}{60}$ & 2 & $\begin{array}{l}15.98 \\
18.33 \\
\end{array}$ & 0.46 & 2.64 & $\begin{array}{l}.87 \\
4.24\end{array}$ & -1.72 & -0.75 & 214.59 & $\frac{0}{0}$ & 0 \\
\hline 60 & 5 & $\begin{array}{l}13.2 \\
13.2\end{array}$ & $\frac{6}{6}$ & 90 & $\frac{1}{3}$ & $\frac{18.33}{6.56}$ & $\frac{1.14}{1.81}$ & $\frac{8.81}{16.65}$ & $\frac{4.24}{10.20}$ & $\frac{4.19}{10.20}$ & $\frac{-0.65}{0.00}$ & $\frac{252.04}{185.15}$ & 0 & 0 \\
\hline 68 & 5 & $\frac{13.2}{13.2}$ & 6 & 120 & $\frac{1}{1}$ & 2.33 & 0.31 & $\begin{array}{l}3.79 \\
\end{array}$ & 1.84 & 1.63 & 0.86 & $\begin{array}{l}162.46 \\
\end{array}$ & & 0 \\
\hline 69 & 5 & 13.2 & 6 & 150 & 1 & 3.89 & 0.89 & 2.90 & 0.66 & 0.46 & 0.48 & 17 & & 0 \\
\hline 70 & 5 & 13.2 & 6 & 180 & 1 & 3.8 & 0.52 & 3.83 & 0.29 & 0.00 & -0.29 & 178.09 & 0 & 0 \\
\hline 71 & $\frac{5}{5}$ & 13.2 & 8 & 0 & 3 & 20.24 & 0.09 & $\begin{array}{l}3.14 \\
8\end{array}$ & 0.60 & 0.00 & 0.60 & 202.63 & & 0 \\
\hline 73 & $\frac{5}{5}$ & $\frac{13.2}{132}$ & $\frac{8}{8}$ & $\frac{30}{60}$ & $\frac{3}{1}$ & $\frac{12.83}{9.51}$ & $\frac{1.58}{0.81}$ & 8. & $\begin{array}{l}7.00 \\
29.47\end{array}$ & $\frac{6.40}{29.42}$ & $\frac{-2.85}{-1.88}$ & $\frac{18 .}{199}$ & & 0 \\
\hline 74 & 5 & 13.2 & 8 & 90 & $\frac{1}{2}$ & 6.4 & 1.84 & 16 & 9.97 & $\begin{array}{c}29.42 \\
9.97\end{array}$ & $\frac{-1.88}{0.02}$ & 184.15 & & 1 \\
\hline 75 & 5 & 13.2 & 8 & 120 & 1 & 4.8 & 0. & 5. & 1. & 1.56 & 1.17 & 16 & & 0 \\
\hline 76 & 5 & 13.2 & 8 & 150 & 1 & 4.8 & 0.2 & 5. & 1.8 & -0.57 & -1.71 & 180.87 & & 0 \\
\hline 77 & 5 & 13.2 & 8 & 180 & 2 & 4.8 & 0.84 & 3.8 & 1.61 & 0.00 & 1.61 & $20 !$ & 0 & 0 \\
\hline $\begin{array}{l}78 \\
79\end{array}$ & $\frac{5}{5}$ & $\begin{array}{l}13.2 \\
13.2\end{array}$ & $\frac{10}{10}$ & $\frac{0}{30}$ & $\frac{3}{2}$ & $\begin{array}{l}8.09 \\
7.73\end{array}$ & $\frac{1.21}{2.51}$ & $\begin{array}{l}0.57 \\
6.34\end{array}$ & $\begin{array}{l}0.38 \\
6.05\end{array}$ & $\begin{array}{l}0.00 \\
1.46\end{array}$ & $\begin{array}{r}-0.38 \\
-5.87 \\
\end{array}$ & 179.62 & $\frac{0}{0}$ & 0 \\
\hline 80 & & 132 & 10 & 60 & $\frac{4}{4}$ & 36 & 2. & 8. & 7.04 & & -4.42 & 293.92 & 0 & 0 \\
\hline 81 & 5 & 13.2 & 10 & 90 & 2 & 5.79 & 1.85 & 15.69 & 9.73 & 9.73 & 0.01 & 185.14 & 0 & 0 \\
\hline $\begin{array}{l}82 \\
83\end{array}$ & $\frac{5}{5}$ & $\frac{13.2}{13.2}$ & $\frac{10}{10}$ & $\frac{120}{150}$ & & $\frac{1 .}{2 .}$ & $\frac{1.4}{0.9}$ & 8. & 3. & $\frac{2 .}{0 .}$ & \begin{tabular}{|l|l|}
2.16 \\
0.85
\end{tabular} & $\frac{170.95}{193.9}$ & & $\frac{0}{0}$ \\
\hline 84 & 5 & 13.2 & $\frac{10}{10}$ & $\frac{100}{180}$ & 2 & 8. & 0.82 & $\begin{array}{l}5.09 \\
5.09\end{array}$ & 1.18 & $\begin{array}{l}0.33 \\
0.00\end{array}$ & $\frac{0.85}{1.18}$ & $\frac{19}{275}$ & 0 & 0 \\
\hline 85 & 6 & 15.3 & 6 & 0 & 4 & 14 & 1. & 1 & 2. & 0.00 & -2.16 & 19 & 0 & 0 \\
\hline 86 & 6 & 15.3 & 6 & 30 & $\frac{4}{4}$ & 19 & 1 & & & 0. & -3 & & 0 & 0 \\
\hline$\frac{87}{88}$ & 6 & $\begin{array}{l}15.3 \\
15.3\end{array}$ & $\frac{6}{6}$ & $\begin{array}{l}60 \\
90\end{array}$ & $\frac{\pi}{4}$ & 1. & 2 & $\begin{array}{l}3.35 \\
15.98 \\
\end{array}$ & $\begin{array}{l}4.24 \\
9.45\end{array}$ & \begin{tabular}{|l|}
-3.69 \\
9.45
\end{tabular} & -2.11 & & 0 & $\frac{1}{0}$ \\
\hline 88 & 6 & $\begin{array}{l}15.3 \\
15.3\end{array}$ & $\frac{6}{6}$ & $\frac{90}{120}$ & & 5. & $\frac{2.05}{0.66}$ & $\begin{array}{c}15.98 \\
3.71\end{array}$ & $\begin{array}{l}9.45 \\
2.09\end{array}$ & $\begin{array}{l}9.45 \\
1.83\end{array}$ & $\frac{-0.06}{1.02}$ & $\frac{184}{162}$ & 0 & $\frac{0}{0}$ \\
\hline 90 & 6 & 15.3 & 6 & 150 & & 2. & 1.22 & 2. & 1.8 & 1. & 1.56 & 172 & & 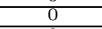 \\
\hline 91 & 6 & 15.3 & 6 & 180 & $\overline{1}$ & 2.7 & 0.87 & 2.8 & 0.8 & 0. & 0. & & & 0 \\
\hline$\frac{92}{93}$ & 6 & $\begin{array}{l}15.3 \\
15.3\end{array}$ & 8 & 0 & $\frac{5}{2}$ & 18. & $\frac{1.4}{1.04}$ & $\begin{array}{ll}4.8 \\
7.6\end{array}$ & 2.5 & 0.00 & $\begin{array}{l}-2.54 \\
-151\end{array}$ & 22 & 0 & $\frac{1}{0}$ \\
\hline $\begin{array}{r}93 \\
94\end{array}$ & 6 & $\begin{array}{l}15.3 \\
153\end{array}$ & 8 & 60 & $\frac{2}{5}$ & $\begin{array}{l}13.41 \\
17.77\end{array}$ & $\frac{1.4}{0.6}$ & 7. & $\begin{array}{l}5.08 \\
1.19\end{array}$ & 4. & $\frac{-1.51}{0.63}$ & $\frac{18}{221}$ & & $\frac{0}{0}$ \\
\hline 05 & 6 & 15.3 & 8 & 0 & & & 0. & & & 9 & & & 0 & 0 \\
\hline 96 & 6 & 15.3 & 8 & 120 & $\begin{array}{lll} & \end{array}$ & 1.96 & 1. & 5. & 2.73 & 2.24 & 1 & & 0 & 0 \\
\hline $\begin{array}{l}97 \\
98\end{array}$ & $\frac{6}{6}$ & $\begin{array}{l}15.3 \\
15.3\end{array}$ & $\frac{8}{8}$ & $\frac{150}{180}$ & 1 & \begin{tabular}{|l|l|}
6.51 \\
2.89
\end{tabular} & $\frac{0 .}{0.8}$ & $\frac{3.8}{3.8}$ & $\begin{array}{l}0.18 \\
0.76\end{array}$ & 0. & $\frac{0 .}{-0}$ & & $\begin{array}{l}0 \\
0\end{array}$ & $\frac{0}{0}$ \\
\hline 98 & 6 & $\begin{array}{l}15.3 \\
15.3\end{array}$ & 8 & 180 & 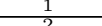 & 2. & 0 & 3 & 0.76 & $\begin{array}{l}0 \\
0 \\
\end{array}$ & 66 & & 0 & $\frac{0}{0}$ \\
\hline 100 & 6 & $\begin{array}{l}1.0 \\
15.3\end{array}$ & 10 & 30 & 1 & 9 & $\frac{0.3}{2.7}$ & 7. & . & 4. & -6 & & & 0 \\
\hline 101 & 6 & 15.3 & 10 & 60 & $\frac{1}{1}$ & 24 & 0. & & & & & & & \\
\hline 102 & 6 & 15.3 & 10 & & & & & & & & & & & 0 \\
\hline 103 & 6 & 15.3 & 10 & 120 & 1 & 2. & 1. & 5.54 & 2 & 2 & 1 & & 0 & 0 \\
\hline $\begin{array}{l}104 \\
105 \\
\end{array}$ & $\begin{array}{ll}6 \\
6\end{array}$ & $\begin{array}{l}15.3 \\
15.3 \\
\end{array}$ & $\frac{10}{10}$ & $\frac{150}{180}$ & 1 & \begin{tabular}{|l|l|}
2.06 \\
4.29 \\
\end{tabular} & \begin{tabular}{|l|l}
1.09 \\
0.69
\end{tabular} & \begin{tabular}{|l|l|}
3.75 \\
4.79 \\
\end{tabular} & \begin{tabular}{|l|l|}
1.06 \\
0.79
\end{tabular} & $\begin{array}{l}0.39 \\
0.00\end{array}$ & \begin{tabular}{|l|l}
0.99 \\
0.79
\end{tabular} & $\begin{array}{l}194.13 \\
249.94\end{array}$ & $\frac{0}{0}$ & $\frac{0}{0}$ \\
\hline
\end{tabular}


Table A.5: LPI+SPA 2.5m low hover full results.

\begin{tabular}{|c|c|c|c|c|c|c|c|c|c|c|c|c|}
\hline Trial & $\begin{array}{l}\text { Sea } \\
\text { State }\end{array}$ & $\begin{array}{l}\text { Wind } \\
\text { Vel. } \\
{[\mathrm{m} / \mathrm{s}]}\end{array}$ & $\begin{array}{l}\text { Ship } \\
\text { Vel. } \\
{[\mathbf{k n n}}\end{array}$ & $\begin{array}{l}\text { Ship } \\
\text { Head. } \\
\text { \& } \\
\text { wind } \\
\text { Dir. } \\
\text { [deg] }\end{array}$ & $\begin{array}{c}\text { Go- } \\
\text { NoGo } \\
\text { State } \\
\text { Change }\end{array}$ & $\begin{array}{c}\text { Total } \\
\text { Land } \\
\text { Time } \\
{[\mathrm{s}]}\end{array}$ & $\begin{array}{c}\text { Impact } \\
\text { Vel. } \\
{[\mathrm{m} / \mathrm{s}]}\end{array}$ & $\begin{array}{c}\text { Land } \\
\text { Angle } \\
\text { [deg] }\end{array}$ & $\begin{array}{c}\text { Ideal } \\
\text { Land } \\
\text { Angle } \\
\text { [deg] }\end{array}$ & $\begin{array}{l}\text { Ship } \\
\text { Roll } \\
\text { a } \\
\text { Land } \\
\text { [deg] }\end{array}$ & $\begin{array}{l}\text { Ship } \\
\text { Pitch } \\
\text { @ } \\
\text { Land } \\
\text { [deg] }\end{array}$ & $\begin{array}{l}\text { Total } \\
\text { Sim. } \\
\text { Time } \\
{[\mathrm{s}]}\end{array}$ \\
\hline$\frac{1}{2}$ & 2 & 3.75 & 6 & $\frac{1}{0}$ & 1 & 3.34 & 0.45 & 0.22 & 0.11 & 0.00 & -0.11 & 160.81 \\
\hline 2 & & $\begin{array}{l}3.75 \\
3.75\end{array}$ & $\frac{6}{6}$ & $\frac{30}{60}$ & & \begin{tabular}{|l|}
3.74 \\
3.06
\end{tabular} & 0.59 & $\begin{array}{l}1.46 \\
0.43\end{array}$ & $\begin{array}{l}0.93 \\
1.26\end{array}$ & $\begin{array}{r}0.93 \\
-1.26 \\
\end{array}$ & $\frac{-0.01}{0.00}$ & $\begin{array}{l}160.77 \\
160.63\end{array}$ \\
\hline$\frac{3}{4}$ & & 3.75 & $\frac{6}{6}$ & $\frac{60}{90}$ & & $\frac{3.06}{3.15}$ & $\frac{0.65}{0.40}$ & 0.43 & $\frac{1.26}{1.11}$ & $\frac{-1.26}{-1.11}$ & $\frac{0.00}{-0.02}$ & $\frac{100.03}{160.56}$ \\
\hline $\begin{array}{l}4 \\
5\end{array}$ & 2 & $\begin{array}{l}3.15 \\
3.75 \\
\end{array}$ & 6 & 120 & $\frac{1}{1}$ & $\frac{3.10}{3.5}$ & 0.54 & 1.20 & $\begin{array}{l}1.11 \\
0.38\end{array}$ & $\begin{array}{r}-1.11 \\
0.33 \\
\end{array}$ & $\begin{array}{r}-0.02 \\
0.18 \\
\end{array}$ & $\begin{array}{l}100.00 \\
161.21\end{array}$ \\
\hline$\frac{6}{67}$ & 2 & 3.75 & 6 & 150 & 1 & 3.45 & 0.53 & 0.99 & 0.21 & 0.09 & 0.20 & 162.06 \\
\hline $\begin{array}{l}7 \\
8\end{array}$ & $\frac{2}{2}$ & $\begin{array}{l}3.75 \\
3.75 \\
\end{array}$ & $\frac{6}{8}$ & 180 & 1 & $\begin{array}{l}3.57 \\
3.62\end{array}$ & 0.50 & 1.26 & $\begin{array}{l}0.01 \\
0.32\end{array}$ & 0.00 & $\begin{array}{r}-0.01 \\
\end{array}$ & $\begin{array}{l}162.5 \\
\end{array}$ \\
\hline 9 & $\frac{2}{2}$ & $\begin{array}{l}3.75 \\
3.75\end{array}$ & $\frac{8}{8}$ & $\frac{0}{30}$ & $\frac{1}{1}$ & $\begin{array}{l}\frac{3.62}{3.65} \\
3.65\end{array}$ & $\frac{0.63}{0.48}$ & $\frac{0.77}{0.66}$ & $\frac{0.32}{0.74}$ & $\begin{array}{r}0.00 \\
-0.74\end{array}$ & $\frac{-0.32}{0.05}$ & $\frac{160.77}{161.34}$ \\
\hline 10 & 2 & 3.75 & 8 & 60 & $\frac{1}{1}$ & 3.45 & $\frac{0.48}{0.56}$ & $\frac{0.00}{1.64}$ & $\frac{0.74}{0.32}$ & $\frac{-0.74}{0.26}$ & 0.05 & 161.44 \\
\hline$\frac{11}{12}$ & $\frac{2}{2}$ & 3.75 & 8 & 90 & 1 & 3.67 & 0.48 & 1.09 & 0.00 & 0.00 & 0.00 & 162.3 \\
\hline 13 & & $\begin{array}{l}3.75 \\
3.75\end{array}$ & $\frac{8}{8}$ & $\frac{120}{150}$ & & $\begin{array}{l}3.39 \\
3.48\end{array}$ & 0.46 & $\begin{array}{l}1.52 \\
1.56\end{array}$ & 0.07 & $\frac{-0.07}{0.01}$ & $\frac{-0.02}{0.06}$ & $\begin{array}{l}163.34 \\
164.79\end{array}$ \\
\hline 14 & $\frac{2}{2}$ & 3.75 & 8 & 180 & & $\begin{array}{l}3.48 \\
3.48\end{array}$ & $\frac{0.53}{0.51}$ & $\begin{array}{l}1.56 \\
1.54\end{array}$ & $\begin{array}{l}0.06 \\
0.09\end{array}$ & 0.01 & $\begin{array}{l}0.06 \\
0.09\end{array}$ & $\frac{164.79}{165.35}$ \\
\hline 15 & 2 & 3.75 & 10 & 0 & & 3.41 & 0.60 & 0.28 & 0.23 & 0.00 & -0.23 & 162.64 \\
\hline 16 & 2 & 3.75 & 10 & 30 & & 3.61 & 0.68 & 0.76 & 0.45 & -0.30 & -0.34 & 162.68 \\
\hline$\frac{17}{18}$ & $\frac{2}{2}$ & $\begin{array}{l}3.75 \\
3.75\end{array}$ & 10 & 60 & & $\begin{array}{l}4.27 \\
3.79 \\
\end{array}$ & 0.36 & 0.45 & 1.09 & -1.09 & -0.04 & 163.58 \\
\hline 19 & $\frac{2}{2}$ & $\begin{array}{l}3.75 \\
3.75\end{array}$ & $\frac{10}{10}$ & $\frac{90}{120}$ & $\frac{1}{1}$ & $\begin{array}{l}3.79 \\
3.55\end{array}$ & 0.56 & $\frac{1.44}{1.82}$ & $\frac{0.60}{0.07}$ & $\begin{array}{c}-0.60 \\
-0.04\end{array}$ & $\begin{array}{l}-0.01 \\
\end{array}$ & $\begin{array}{l}164.74 \\
166.2\end{array}$ \\
\hline 20 & 2 & 3.75 & 10 & 150 & 1 & 3.77 & $\frac{0.45}{0.43}$ & $\frac{1.82}{2.69}$ & $\frac{0.07}{0.42}$ & $\frac{-0.04}{-0.16}$ & $\frac{-0.06}{-0.39}$ & $\frac{166.2}{168.24}$ \\
\hline 21 & 2 & 3.75 & 10 & 180 & 1 & 3.49 & 0.45 & $\frac{2.09}{2.48}$ & 0.42 & $\frac{-0.10}{0.00}$ & $\frac{-0.59}{0.06}$ & 160.24 \\
\hline 22 & 3 & 6.4 & 6 & 0 & & 2.79 & 0.28 & 1.12 & 0.11 & 0.00 & -0.11 & 160.66 \\
\hline & 3 & 6.4 & 6 & 30 & 1 & 3.34 & 0.72 & 3.66 & 2.73 & 2.73 & 0.02 & 164.57 \\
\hline 24 & & 6.4 & 6 & 60 & 1 & 3.08 & 0.26 & 5.32 & 1.07 & 0.84 & 0.67 & 167.61 \\
\hline 25 & $\frac{3}{3}$ & 6.4 & 6 & 90 & 1 & $\begin{array}{l}2.07 \\
3.21\end{array}$ & 0.50 & 5.05 & 2.07 & 2.07 & 0.05 & 159.64 \\
\hline$\frac{26}{27}$ & $\begin{array}{l}3 \\
3\end{array}$ & $\frac{0.4}{6.4}$ & $\frac{0}{6}$ & $\frac{120}{150}$ & & $\begin{array}{r}3.21 \\
3.09\end{array}$ & $\frac{0.52}{0.51}$ & $\begin{array}{l}2.67 \\
1.42\end{array}$ & $\begin{array}{l}1.09 \\
0.40\end{array}$ & $\begin{array}{l}0.96 \\
0.19\end{array}$ & $\frac{0.52}{0.35}$ & $\begin{array}{l}161.76 \\
163.78\end{array}$ \\
\hline 28 & 3 & 6.4 & 6 & 180 & & 3.46 & 0.52 & 1.57 & 0.02 & 0.00 & 0.02 & 165.41 \\
\hline 29 & $\frac{3}{3}$ & 6.4 & 8 & 0 & 1 & $\begin{array}{l}4.32 \\
2.84\end{array}$ & 0.90 & $\frac{2.02}{3.79}$ & 1.27 & 0.00 & -1.28 & 165.43 \\
\hline 31 & $\frac{3}{3}$ & $\begin{array}{l}6.4 \\
6.4\end{array}$ & $\frac{8}{8}$ & $\frac{30}{60}$ & 1 & $\frac{2.84}{3.7}$ & $\frac{0.81}{0.90}$ & $\frac{3.79}{4.75}$ & $\begin{array}{l}3.49 \\
1.57\end{array}$ & $\begin{array}{l}3.11 \\
1.56\end{array}$ & $\frac{-1.58}{-0.19}$ & $\frac{163.23}{161.09}$ \\
\hline 32 & 3 & 6.4 & 8 & 90 & $\frac{1}{1}$ & 3.82 & 0.43 & 1.27 & 0.16 & -0.16 & -0.01 & 162.27 \\
\hline$\frac{33}{34}$ & $\begin{array}{l}3 \\
3\end{array}$ & \begin{tabular}{|l|l|}
6.4 \\
6.4
\end{tabular} & $\frac{8}{8}$ & 120 & $\frac{1}{1}$ & \begin{tabular}{|l|l|}
3.75 \\
3.39
\end{tabular} & 0.34 & $\begin{array}{l}2.84 \\
2.20\end{array}$ & $\begin{array}{l}0.94 \\
\end{array}$ & $\begin{array}{l}-0.77 \\
0.01\end{array}$ & $\begin{array}{r}-0.55 \\
\end{array}$ & $\begin{array}{l}164.7 \\
168\end{array}$ \\
\hline 35 & & $\frac{6.4}{6.4}$ & & $\frac{150}{180}$ & $\frac{1}{1}$ & $\begin{array}{l}3.39 \\
3.8\end{array}$ & $\frac{0.47}{0.44}$ & $\frac{2.20}{3.09}$ & 0.22 & $\frac{0.01}{0.00}$ & $\frac{0.12}{-0.23}$ & $\frac{168}{170.43}$ \\
\hline 36 & & 6.4 & 10 & 0 & & 2.93 & $\frac{0.44}{0.19}$ & 1.83 & 2.06 & $\frac{0.00}{0.00}$ & $\frac{-0.20}{2.00}$ & 160.456 \\
\hline 37 & & 6.4 & 10 & 30 & & 7.94 & 1.08 & 2.46 & 2.25 & 0.53 & -2.19 & 178.33 \\
\hline 38 & 3 & 6.4 & 10 & 60 & 4 & 6.88 & 0.71 & 3.48 & 3.24 & -3.12 & -0.87 & 200.87 \\
\hline$\frac{39}{40}$ & & $\begin{array}{l}6.4 \\
6.4\end{array}$ & 10 & $\frac{90}{120}$ & & $\begin{array}{r}4.33 \\
4.59 \\
\end{array}$ & $\begin{array}{l}0.75 \\
0.49\end{array}$ & $\frac{1.94}{3.18}$ & $\begin{array}{l}1.88 \\
0.93\end{array}$ & $\frac{-1.88}{-0.80}$ & $\begin{array}{c}-0.03 \\
-0.48\end{array}$ & 165.06 \\
\hline 41 & & 6.4 & 10 & 150 & $\frac{1}{1}$ & $\begin{array}{l}4.59 \\
3.23\end{array}$ & $\frac{0.49}{0.64}$ & $\begin{array}{l}2.18 \\
2.99\end{array}$ & 0.81 & $\frac{-0.80}{0.36}$ & $\frac{-0.48}{0.72}$ & $\begin{array}{l}168.9 \\
173.62\end{array}$ \\
\hline 42 & & 6.4 & 10 & 180 & 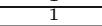 & 3.09 & 0.70 & 3.23 & 0.56 & 0.00 & 0.56 & 176.94 \\
\hline 43 & & 9.4 & 6 & 0 & 7 & 4.43 & 0.71 & 3.22 & 1.70 & 0.00 & 1.70 & 197.2 \\
\hline 44 & 4 & $\begin{array}{l}9.4 \\
9.4\end{array}$ & 6 & 30 & $\frac{1}{3}$ & $\begin{array}{l}2.98 \\
871\end{array}$ & 1.00 & 4.18 & 0.38 & 0.38 & 0.05 & 202.39 \\
\hline$\frac{40}{46}$ & $\frac{4}{4}$ & $\begin{array}{l}9.4 \\
9.4\end{array}$ & $\frac{6}{6}$ & $\frac{60}{90}$ & $\frac{3}{6}$ & $\begin{array}{l}8.71 \\
18.94\end{array}$ & $\frac{0.80}{0.39}$ & $\begin{array}{l}5.97 \\
7.60\end{array}$ & $\begin{array}{l}\frac{2.66}{2.74} \\
2.74\end{array}$ & $\frac{-1.80}{2.74}$ & $\frac{-1.95}{0.02}$ & $\frac{176.88}{315.25}$ \\
\hline 47 & 4 & 9.4 & 6 & 120 & Th & $\frac{10.11}{3.11}$ & 0.25 & 2.28 & 0.32 & $\frac{2.14}{-0.26}$ & $\frac{0.02}{-0.18}$ & 163.08 \\
\hline 48 & 4 & 9.4 & 6 & 150 & 1 & 4.49 & 0.52 & 2.96 & 0.03 & -0.01 & 0.03 & 170.36 \\
\hline 49 & & $\begin{array}{l}9.4 \\
9.4\end{array}$ & 6 & 180 & $\frac{1}{6}$ & 3.97 & 0.49 & 3.10 & 0.00 & 0.00 & 0.01 & 174.6 \\
\hline 51 & $\frac{4}{4}$ & $\frac{3.4}{9.4}$ & 8 & 30 & 4 & 7.19 & $\begin{array}{l}0.31 \\
0.99\end{array}$ & $\frac{1.48}{4.41}$ & $\frac{0.39}{0.85}$ & $\frac{0.00}{0.84}$ & $\frac{-0.39}{0.09}$ & $\frac{197.46}{184.96}$ \\
\hline 52 & & & 8 & 60 & & 3.65 & $\frac{1.09}{1.09}$ & 8.79 & $\frac{2.64}{2.64}$ & $\frac{2.62}{2.62}$ & 0.32 & 161.48 \\
\hline 53 & 4 & 9.4 & 8 & 90 & 4 & 10.03 & 0.91 & 4.79 & 0.70 & 0.69 & 0.02 & 205.06 \\
\hline 54 & 4 & 9.4 & 8 & 120 & 1 & 4.46 & 0.61 & 2.90 & 0.33 & 0.23 & 0.24 & 167.41 \\
\hline 55 & 4 & $\begin{array}{l}9.4 \\
9.4\end{array}$ & 8 & 150 & 1 & $\begin{array}{l}2.52 \\
\end{array}$ & 0.68 & $\begin{array}{l}2.97 \\
3.18\end{array}$ & 1.25 & 0.40 & 1.18 & 99 \\
\hline 56 & $\frac{4}{4}$ & $\begin{array}{l}9.4 \\
9.4\end{array}$ & $\frac{8}{10}$ & $\frac{180}{0}$ & $\frac{1}{1}$ & $\begin{array}{l}2.78 \\
3.15 \\
\end{array}$ & 0.71 & 3.18 & $\begin{array}{l}0.69 \\
1.50\end{array}$ & $\begin{array}{l}0.00 \\
0.00\end{array}$ & $\begin{array}{l}0.69 \\
1.50\end{array}$ & $\begin{array}{l}185.43 \\
163.5\end{array}$ \\
\hline 58 & 4 & $\begin{array}{l}9.4 \\
9.4\end{array}$ & 10 & 30 & 4 & $\begin{array}{l}0.15 \\
10.5\end{array}$ & 0.95 & 0.79 & $\begin{array}{l}1.00 \\
0.76\end{array}$ & $\frac{0.00}{-0.52}$ & $\begin{array}{l}1.00 \\
-0.56\end{array}$ & $\begin{array}{l}103.5 \\
192.49\end{array}$ \\
\hline 59 & 4 & 9.4 & 10 & 60 & 6 & 15.23 & 1.71 & 5.56 & 3.59 & 2.86 & -2.17 & 294.26 \\
\hline $\begin{array}{l}60 \\
61 \\
\end{array}$ & $\frac{4}{4}$ & $\begin{array}{r}9.4 \\
9.4\end{array}$ & $\frac{10}{10}$ & $\frac{90}{120}$ & 2 & $\begin{array}{l}3.43 \\
2.32 \\
\end{array}$ & $\begin{array}{l}0.51 \\
100\end{array}$ & $\begin{array}{l}6.91 \\
5.94\end{array}$ & \begin{tabular}{|l|l|}
2.07 \\
2.18
\end{tabular} & $\begin{array}{l}4.07 \\
1.74\end{array}$ & 0.08 & $\begin{array}{l}166.58 \\
17091\end{array}$ \\
\hline 62 & 4 & $\begin{array}{l}9.4 \\
9.4\end{array}$ & 10 & $\frac{120}{150}$ & 1 & 2.52 & 0.43 & $\begin{array}{l}0.94 \\
3.56\end{array}$ & $\frac{2.18}{0.11}$ & $\frac{1.14}{-0.05}$ & $\frac{1.22}{-0.10}$ & $\begin{array}{l}189.03 \\
189\end{array}$ \\
\hline 63 & 4 & 9.4 & 10 & 180 & 1 & 2.82 & 0.64 & 5.08 & 0.70 & 0.00 & 0.70 & 319.21 \\
\hline 64 & 5 & $\frac{13.2}{13}$ & 6 & 0 & $\frac{13}{2}$ & 54.75 & 0.55 & 0.03 & 2.09 & 0.00 & -2.00 & 33 \\
\hline$\frac{65}{66}$ & & $\frac{13.2}{13.2}$ & $\frac{0}{6}$ & $\frac{30}{60}$ & $\frac{2}{1}$ & $\begin{array}{l}15.98 \\
18.33\end{array}$ & 0.46 & $\begin{array}{l}2.64 \\
8.81\end{array}$ & 1.87 & -1.72 & -0.75 & 214.59 \\
\hline 67 & 5 & 13.2 & 6 & 90 & $\frac{1}{3}$ & $\frac{1.50}{6.56}$ & $\frac{1.14}{1.81}$ & $\frac{.61}{16.65}$ & $\frac{4.24}{10.20}$ & $\frac{4.19}{10.20}$ & $\begin{array}{l}-0.00 \\
0.00\end{array}$ & 185 \\
\hline 68 & 5 & 13.2 & 6 & 120 & $\frac{1}{1}$ & 2.33 & $\frac{1.01}{0.31}$ & $\begin{array}{l}10.79 \\
\end{array}$ & 1.84 & 1.63 & 0.86 & 162.46 \\
\hline 69 & 5 & 13.2 & 6 & 150 & 1 & & & & & 0.46 & & 172.1 \\
\hline 70 & 5 & 13.2 & 6 & 180 & $\overline{1}$ & 3.8 & 0.52 & 3.83 & 0.29 & 0.00 & -0.29 & 178.09 \\
\hline$\frac{11}{72}$ & $\frac{5}{5}$ & $\frac{13.2}{13.2}$ & $\frac{8}{8}$ & $\frac{0}{30}$ & $\frac{3}{3}$ & $\begin{array}{l}20.24 \\
12.83\end{array}$ & $\frac{0.09}{1.58}$ & $\begin{array}{l}3.14 \\
8.33\end{array}$ & $\frac{0.60}{7.00}$ & $\frac{0.00}{6.40}$ & $\begin{array}{l}0.60 \\
-2.85 \\
\end{array}$ & $\frac{202}{184}$ \\
\hline 73 & 5 & 13.2 & 8 & 60 & 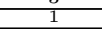 & 9. & 0. & $\begin{array}{l}0.05 \\
13.73\end{array}$ & $\frac{1.0}{29 .}$ & $\frac{0.40}{29.42}$ & $\frac{-2.00}{-1.88}$ & $\frac{184}{194}$ \\
\hline 74 & 5 & 13.2 & 8 & 90 & 2 & 6.4 & 1.84 & 16.06 & 9.97 & 9.97 & 0.02 & 185.15 \\
\hline 76 & & $\begin{array}{l}\frac{13.2}{13.2} \\
13.2\end{array}$ & 8 & $\frac{120}{150}$ & & $\begin{array}{r}4.82 \\
4.88 \\
\end{array}$ & 0.8 & $\begin{array}{r}5.35 \\
5.21 \\
\end{array}$ & 1.5 & $\begin{array}{l}1.56 \\
0.57\end{array}$ & 1.17 & \\
\hline 76 & 5 & $\begin{array}{l}13.2 \\
13.2\end{array}$ & 8 & $\frac{150}{180}$ & & $\begin{array}{l}4.88 \\
485\end{array}$ & 0.23 & $\frac{5.21}{3.81}$ & 1.80 & $\frac{-0.57}{0.07}$ & $\frac{-1.71}{1.61}$ & $\frac{180.87}{20938}$ \\
\hline & & & 10 & 0 & & $\begin{array}{l}.09 \\
\end{array}$ & 1.21 & 0.5 & & 0.00 & -0.38 & 179.62 \\
\hline 79 & 5 & 13.2 & 10 & 30 & 2 & 7.73 & 2.51 & 6.34 & 6.05 & 1.46 & -5.87 & 178.44 \\
\hline 80 & 5 & 13.2 & 10 & 60 & $\frac{4}{2}$ & 36 & 2. & 8 & 7. & $\begin{array}{l}5.49 \\
0.72\end{array}$ & $\frac{-4.42}{-0.42}$ & 29 \\
\hline$\frac{81}{82}$ & 5 & $\begin{array}{l}3.2 \\
13.2\end{array}$ & 10 & 90 & 2 & $\begin{array}{l}5.79 \\
1.58\end{array}$ & 1.41 & $\frac{15.69}{8.88}$ & $\begin{array}{l}9.73 \\
3.58\end{array}$ & \begin{tabular}{|l|}
9.73 \\
2.86
\end{tabular} & \begin{tabular}{|l|l|}
0.01 \\
2.16
\end{tabular} & $\begin{array}{l}185.14 \\
170.95\end{array}$ \\
\hline$\frac{02}{83}$ & 5 & $\frac{10.2}{13.2}$ & 10 & 150 & & $\frac{1.0}{2.2}$ & & 3. & 0. & 2.86 & $\frac{2}{0 .}$ & $\frac{11}{19}$ \\
\hline 84 & 5 & 13.2 & 10 & 180 & $\frac{2}{2}$ & 8. & 0. & 5.09 & 1. & 0. & 1. & \\
\hline $\begin{array}{l}85 \\
86\end{array}$ & 6 & $\begin{array}{l}15.3 \\
\end{array}$ & 6 & 0 & & 14.46 & 1.50 & 1.67 & 2.16 & 0.00 & -2.16 & 196.03 \\
\hline $\begin{array}{l}86 \\
87\end{array}$ & 6 & $\begin{array}{l}15.3 \\
15.3\end{array}$ & 6 & $\frac{30}{60}$ & & $\frac{19.82}{1.57}$ & $\frac{1.39}{1.41}$ & $\begin{array}{l}2.64 \\
3.35\end{array}$ & $\frac{3.51}{4.24}$ & $\begin{array}{r}0.18 \\
-3.369\end{array}$ & & \\
\hline 88 & $\frac{0}{6}$ & $\frac{15.5}{15.3}$ & $\frac{0}{6}$ & ov & & 1. & $\frac{1.4}{20}$ & 3 & $\frac{4.2}{9}$ & $\frac{-3 .}{9}$ & $\begin{array}{l}-2.11 \\
\end{array}$ & \\
\hline 89 & $\frac{0}{6}$ & $\frac{15.5}{15.3}$ & $\frac{0}{6}$ & $\frac{90}{120}$ & $\frac{4}{1}$ & $\frac{3 .}{1.9}$ & $\frac{2.05}{0.66}$ & 3.90 & $\begin{array}{l}3.400 \\
2.09\end{array}$ & $\frac{3.45}{1.83}$ & $\frac{-0.06}{1.02}$ & $\frac{184.95}{162.61}$ \\
\hline 90 & 6 & 15.3 & 6 & 150 & $\sqrt{2}$ & 2.16 & 1.2 & 2.80 & 1.85 & 1.00 & 1.56 & 172.75 \\
\hline 91 & 6 & 15.3 & 6 & 180 & 1 & 2.74 & $0 . \varepsilon$ & 2.8 & 0.81 & 0.1 & 0.8 & \\
\hline 92 & 6 & 15.3 & 8 & 0 & 5 & 18.92 & 1.47 & 4.81 & 2.54 & 0.00 & -2.54 & 224.61 \\
\hline$\frac{93}{94}$ & 6 & $\begin{array}{l}15.3 \\
15.3\end{array}$ & 8 & 30 & $\frac{2}{5}$ & $\begin{array}{l}13.41 \\
17.77\end{array}$ & 0 & 7.68 & 5.0 & 4. & -1 & 18 \\
\hline$\frac{34}{95}$ & $\frac{0}{6}$ & & 8 & & & & & & $\frac{1}{9}$ & & & \\
\hline 96 & 6 & $\frac{1.3}{15.3}$ & 8 & 120 & 3 & 4.89 & 2.08 & $\begin{array}{l}15.0 \% \\
5.99\end{array}$ & 9.10 & $\frac{9.10}{2.24}$ & $\frac{-0.08}{1.57}$ & $\begin{array}{l}184.92 \\
16709\end{array}$ \\
\hline 97 & 6 & $\frac{15.3}{15.3}$ & 8 & 150 & & $\frac{1.5}{6.5}$ & 0.8 & $\frac{3.8}{3.8}$ & 0. & 2. & $\frac{1}{0}$ & 18 \\
\hline 98 & 6 & 15.3 & 8 & 180 & 1 & 2. & 0 & 3 & 0 & 0 & & \\
\hline 99 & 6 & 15.3 & 10 & 0 & 2 & 8.2 & 0.34 & 1.56 & 0.24 & 0.00 & -0.24 & 178 \\
\hline 100 & $\begin{array}{ll}6 \\
6\end{array}$ & $\begin{array}{l}15.3 \\
15.3\end{array}$ & 10 & $\begin{array}{l}30 \\
60\end{array}$ & $\frac{1}{1}$ & $\begin{array}{c}9.7 \\
24.44\end{array}$ & 2.75 & 7.70 & $\begin{array}{l}7.97 \\
6.70\end{array}$ & $\begin{array}{l}4.22 \\
6.59 \\
6.59\end{array}$ & -6.77 & $\begin{array}{l}184.45 \\
252.01\end{array}$ \\
\hline 102 & 6 & 15.3 & 10 & 90 & & 5.24 & 2. & 15 & 8.85 & 8. & -0 & $\frac{20201}{184.91}$ \\
\hline 103 & 6 & 15.3 & 10 & 120 & +2 & 2.08 & 1.13 & 5.54 & 2.88 & 2.51 & 1.41 & 187.27 \\
\hline 104 & $\frac{6}{6}$ & 15.3 & 10 & 180 & 1 & 429 & 0.69 & 479 & $\begin{array}{l}1.06 \\
0.79\end{array}$ & 000 & 0.79 & $\frac{194.13}{249.94}$ \\
\hline
\end{tabular}


Table A.6: $\mathrm{LPI}+\mathrm{SPA}+\mathrm{AHC} 2.5 \mathrm{~m}$ low hover full results.

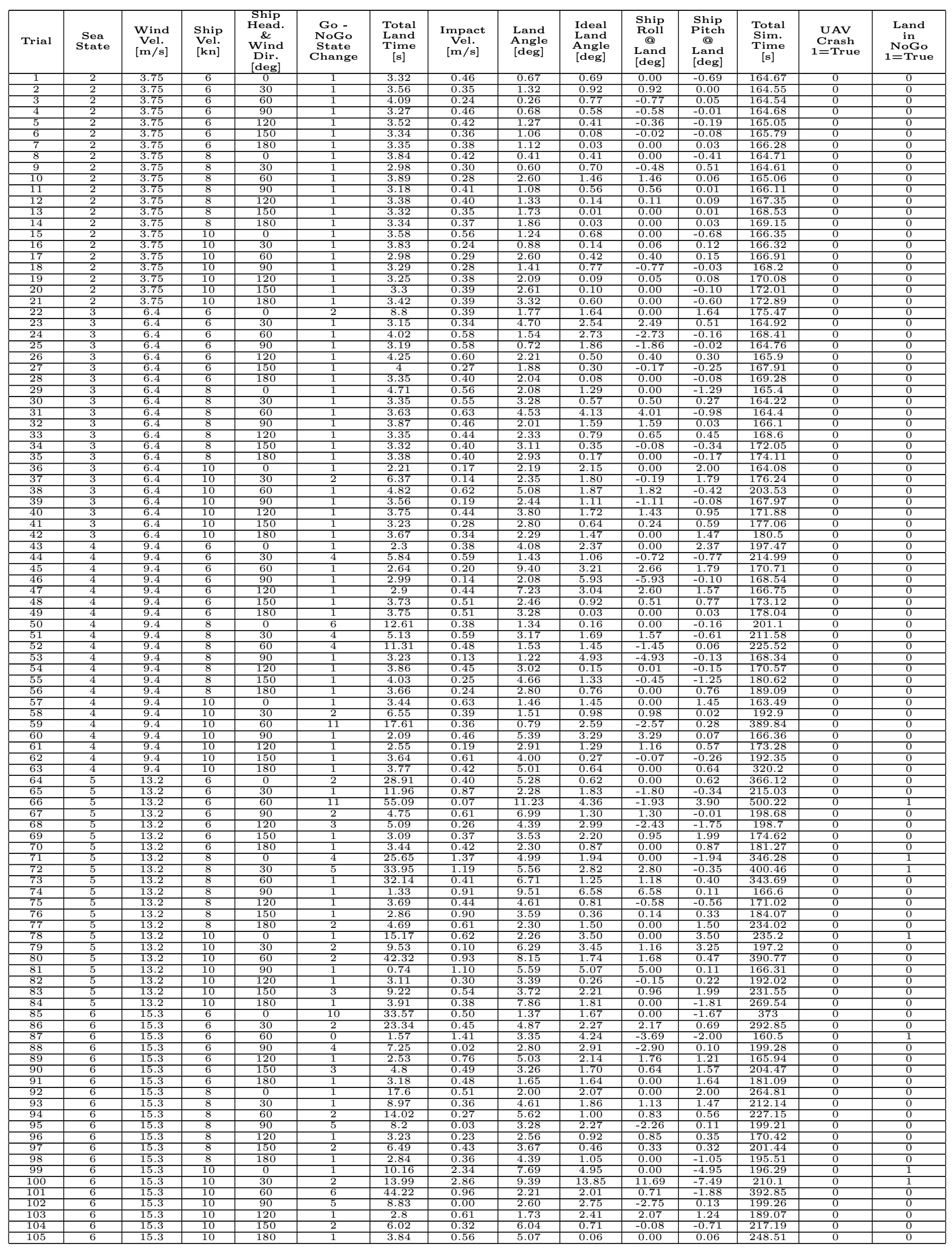


Table A.7: LPI+AHC 2.5m low hover full results.

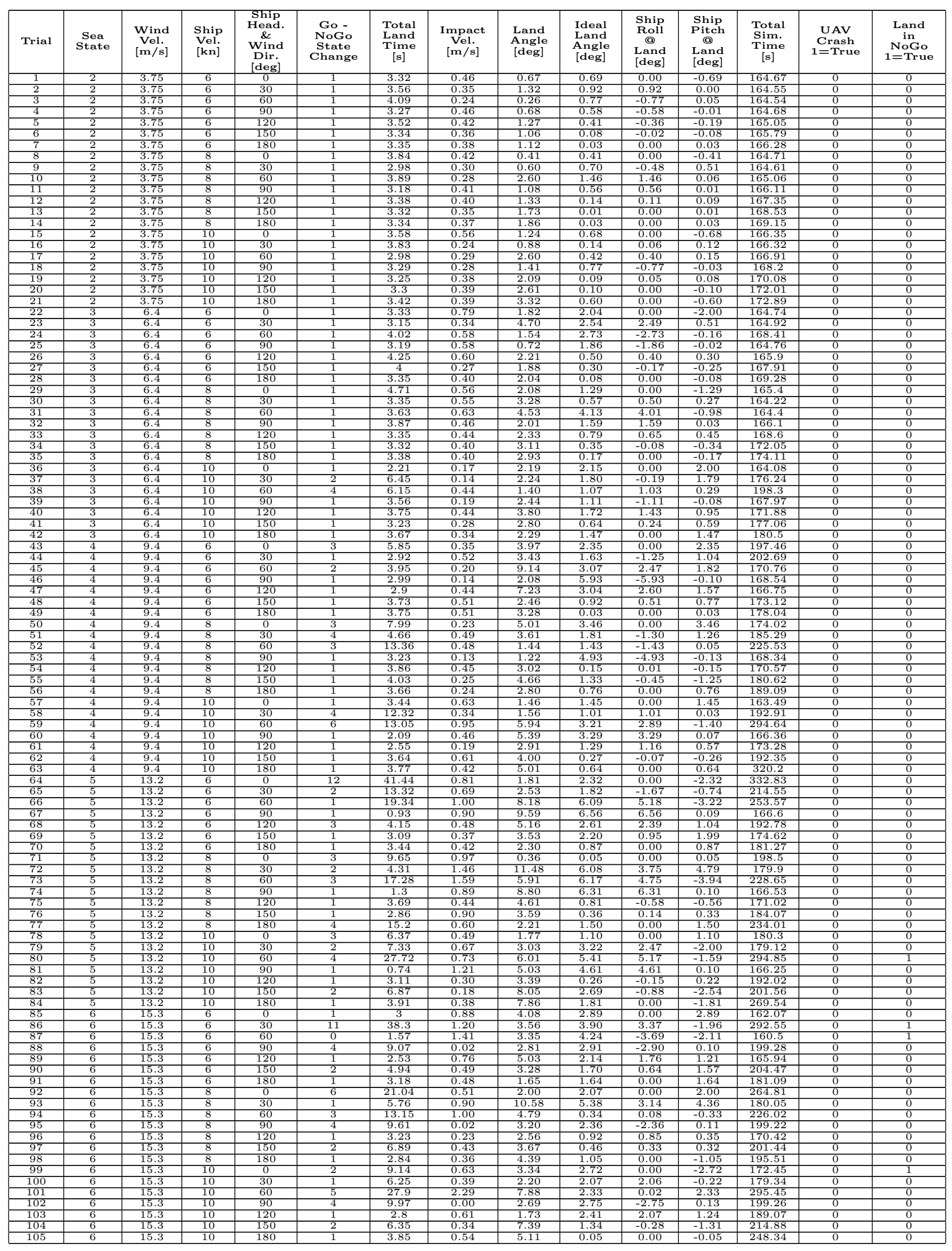


Appendix B

Full Results - Low Hover, 5 m 
Table B.1: Unregulated 5m low hover full results.

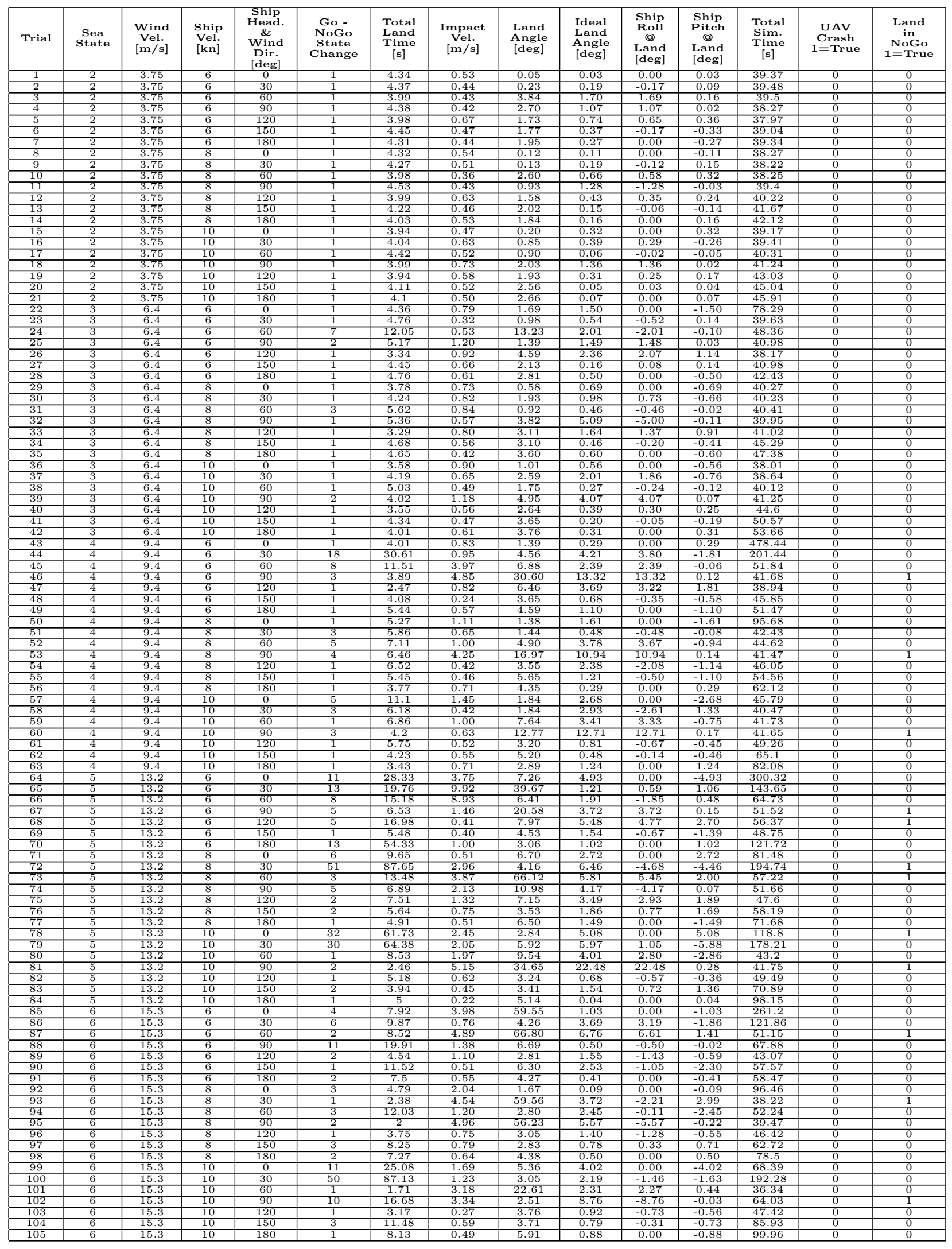


Table B.2: SPA 5m low hover full results.

\begin{tabular}{|c|c|c|c|c|c|c|c|c|}
\hline Trial & $\begin{array}{c}\text { Sea } \\
\text { State }\end{array}$ & $\begin{array}{l}\text { Wind } \\
\text { Vel. } \\
{[\mathrm{m} / \mathrm{s}]}\end{array}$ & $\begin{array}{l}\text { Ship } \\
\text { Vel } \\
\text { [kn] }\end{array}$ & $\begin{array}{l}\text { Ship } \\
\text { Head. } \\
\text { \& } \\
\text { wind } \\
\text { Dir. } \\
\text { [deg] }\end{array}$ & $\begin{array}{c}\text { Go- } \\
\text { NoGo } \\
\text { State } \\
\text { Change }\end{array}$ & $\begin{array}{c}\text { Total } \\
\text { Land } \\
\text { Time } \\
{[\mathrm{s}]}\end{array}$ & $\begin{array}{c}\text { Impact } \\
\text { Vel } \\
{[\mathbf{m} / \mathrm{s}]}\end{array}$ & $\begin{array}{c}\text { Land } \\
\text { Angle } \\
\text { [deg] }\end{array}$ \\
\hline$\frac{1}{2}$ & $\begin{array}{l}\frac{2}{2} \\
2\end{array}$ & $\begin{array}{l}3.75 \\
3.75 \\
\end{array}$ & $\frac{6}{6}$ & $\frac{0}{30}$ & $\frac{1}{1}$ & 4.35 & 0.57 & 0.58 \\
\hline 3 & 2 & $\begin{array}{l}3.15 \\
3.75 \\
\end{array}$ & $\frac{0}{6}$ & $\begin{array}{l}30 \\
60\end{array}$ & 1 & $\frac{4.34}{4.15}$ & $\begin{array}{l}0.61 \\
0.42\end{array}$ & $\frac{1.11}{0.11}$ \\
\hline 4 & 2 & & 6 & 90 & & 4.11 & 0.44 & 0.72 \\
\hline 5 & 2 & 3.75 & 6 & 120 & 1 & 3.85 & 0.49 & 0.77 \\
\hline 6 & 2 & 3.75 & 6 & 150 & 1 & 4.28 & 0.49 & 1.24 \\
\hline 7 & 2 & $\begin{array}{l}3.75 \\
\end{array}$ & 6 & 180 & 1 & 4.21 & 0.51 & $\begin{array}{l}.33 \\
\end{array}$ \\
\hline$\frac{8}{9}$ & $\frac{2}{2}$ & $\begin{array}{r}3.75 \\
3.75\end{array}$ & $\begin{array}{l}8 \\
8\end{array}$ & $\begin{array}{l}0 \\
30\end{array}$ & 1 & $\begin{array}{l}3.96 \\
3.98\end{array}$ & $\begin{array}{l}0.43 \\
0.74\end{array}$ & 0.62 \\
\hline$\frac{9}{10}$ & $\frac{2}{2}$ & $\begin{array}{r}3.75 \\
3.75\end{array}$ & 8 & 60 & $\frac{1}{1}$ & $\begin{array}{r}3.98 \\
4.33\end{array}$ & 0.74 & $\begin{array}{l}2.30 \\
3.05\end{array}$ \\
\hline 11 & 2 & $\begin{array}{l}3.75 \\
275\end{array}$ & 8 & 90 & 1 & 4.29 & 0.50 & 1.10 \\
\hline 12 & 2 & 3.75 & 8 & 120 & & 4.13 & 0.49 & 1.62 \\
\hline$\frac{13}{14}$ & $\frac{2}{2}$ & $\begin{array}{r}3.75 \\
33.75\end{array}$ & $\frac{8}{8}$ & $\begin{array}{l}150 \\
180\end{array}$ & $\frac{1}{1}$ & $\begin{array}{l}4.27 \\
4.23\end{array}$ & 0.51 & 1.99 \\
\hline & & 3.75 & & 0 & & $\frac{4.25}{4.21}$ & 0.40 & 0.13 \\
\hline 16 & & 3.75 & 10 & 30 & $\frac{1}{1}$ & 3.7 & 0.43 & $\frac{0.00}{1.60}$ \\
\hline 17 & 2 & 3.75 & 10 & 60 & 1 & 4.11 & 0.63 & $\begin{array}{l}1.46 \\
\end{array}$ \\
\hline 18 & 2 & $\begin{array}{l}3.75 \\
275\end{array}$ & 10 & 90 & 1 & 4.25 & 0.51 & 2.43 \\
\hline 19 & $\frac{2}{2}$ & $\begin{array}{l}3.75 \\
3.75\end{array}$ & 10 & 120 & 1 & $\begin{array}{l}3.91 \\
\end{array}$ & 0.48 & 1.97 \\
\hline$\frac{20}{21}$ & $\frac{2}{2}$ & $\begin{array}{r}3.75 \\
.75\end{array}$ & $\begin{array}{l}10 \\
10\end{array}$ & $\begin{array}{l}\frac{150}{180} \\
180\end{array}$ & $\frac{1}{1}$ & $\begin{array}{l}3.95 \\
.08\end{array}$ & $\begin{array}{l}0.56 \\
0.58\end{array}$ & $\frac{2.10}{2.61}$ \\
\hline 22 & 3 & 6.4 & $\frac{10}{6}$ & $\frac{180}{0}$ & 1 & $\begin{array}{l}4.08 \\
3.82\end{array}$ & $\begin{array}{l}0.58 \\
1.00\end{array}$ & $\frac{2.01}{2.17}$ \\
\hline 23 & 3 & 6.4 & 6 & 30 & 1 & 4.47 & 0.80 & 3.99 \\
\hline 24 & & 6.4 & 6 & 60 & 1 & 3.95 & 0.22 & 1.48 \\
\hline 25 & 3 & & 6 & 90 & 1 & 4.02 & 0.28 & 0.65 \\
\hline 27 & $\frac{3}{3}$ & $\begin{array}{l}6.4 \\
6\end{array}$ & 6 & 120 & 1 & $\begin{array}{l}3.94 \\
\end{array}$ & 0.15 & $\begin{array}{l}4.18 \\
205\end{array}$ \\
\hline 28 & $\frac{3}{3}$ & $\frac{0.4}{6.4}$ & $\frac{6}{6}$ & $\frac{150}{180}$ & $\frac{1}{1}$ & $\frac{4.27}{4.05}$ & $\frac{0.58}{0.57}$ & $\frac{2.05}{2.09}$ \\
\hline 29 & 3 & 6.4 & 8 & 0 & 1 & 5.12 & 0.41 & 0.47 \\
\hline 30 & 3 & 6.4 & 8 & 30 & 7 & 7.54 & 0.98 & 2.51 \\
\hline 1 & & 6.4 & 8 & 60 & 4 & 9.77 & 0.96 & 6.09 \\
\hline 32 & 3 & 6.4 & 8 & 90 & 1 & 4.56 & 0.50 & 1.67 \\
\hline 33 & 3 & 6.4 & 8 & 120 & 1 & 4.26 & 0.53 & 2.33 \\
\hline$\frac{34}{35}$ & & 6.4 & 8 & $\begin{array}{l}150 \\
180\end{array}$ & 1 & $\begin{array}{l}3.85 \\
\end{array}$ & 0.01 & $\frac{2.24}{2}$ \\
\hline 36 & 3 & $\begin{array}{l}0.4 \\
6.4\end{array}$ & $\frac{8}{10}$ & $\frac{180}{0}$ & $\frac{1}{1}$ & $\begin{array}{r}3.9 \\
4.16\end{array}$ & 0.54 & $\begin{array}{l}2.40 \\
1.48\end{array}$ \\
\hline 37 & 3 & 6.4 & 10 & 30 & 2 & 5.92 & 0.79 & 0.45 \\
\hline 38 & & 6.4 & 10 & 60 & 1 & 3.53 & 0.55 & 7.13 \\
\hline 39 & 3 & 6.4 & 10 & 90 & 1 & 4.38 & 0.54 & 5.63 \\
\hline 40 & 3 & 6.4 & 10 & 120 & 1 & 5.41 & 0.41 & 3.65 \\
\hline $\begin{array}{ll}41 \\
4\end{array}$ & & 6.4 & 10 & $\frac{150}{180}$ & $\frac{1}{1}$ & 4.69 & 0.44 & 3.93 \\
\hline$\frac{42}{43}$ & $\begin{array}{l}3 \\
4\end{array}$ & $\begin{array}{l}6.4 \\
9.4\end{array}$ & $\frac{10}{6}$ & $\frac{100}{0}$ & 1 & $\begin{array}{l}4.66 \\
3.27\end{array}$ & $\frac{0.30}{1.00}$ & $\frac{4.43}{0.06}$ \\
\hline 44 & 4 & 9.4 & 6 & 30 & 3 & 3.86 & 4.35 & 8.61 \\
\hline 45 & 4 & 9.4 & 6 & 60 & 6 & 15.37 & 0.82 & 4.88 \\
\hline 46 & 4 & 9.4 & 6 & 90 & 11 & 38.87 & 0.56 & 7.47 \\
\hline 47 & 4 & 9.4 & 6 & 120 & 3 & 7.21 & 1.00 & 5.08 \\
\hline 48 & 4 & $\begin{array}{l}9.4 \\
\end{array}$ & 6 & 150 & 1 & 4.18 & 0.13 & 3.43 \\
\hline $\begin{array}{l}49 \\
50\end{array}$ & 4 & $\begin{array}{l}9.4 \\
9.4\end{array}$ & $\begin{array}{l}6 \\
8\end{array}$ & 180 & 1 & 3.67 & $\frac{0.66}{2.25}$ & $\begin{array}{l}2.56 \\
1.75\end{array}$ \\
\hline 51 & 4 & $\begin{array}{l}9.4 \\
9.4\end{array}$ & 8 & 0 & 5 & $\frac{8}{13.19}$ & $\begin{array}{l}2.35 \\
0.90\end{array}$ & $\begin{array}{l}1.15 \\
3.26\end{array}$ \\
\hline 2 & 4 & 9.4 & 8 & 60 & & 14.14 & 0.57 & \\
\hline 53 & 4 & 9.4 & 8 & 90 & 11 & 37.35 & 0.66 & 6.88 \\
\hline 54 & & 9.4 & 8 & 120 & 1 & 3.75 & 0.62 & 3.39 \\
\hline 55 & 4 & 9.4 & 8 & 150 & 1 & 4.09 & 0.74 & 3.03 \\
\hline $\begin{array}{l}56 \\
57\end{array}$ & 4 & \begin{tabular}{|l|}
9.4 \\
9.4
\end{tabular} & 88 & 180 & $\begin{array}{l}1 \\
3\end{array}$ & 5.33 & $\begin{array}{l}0.44 \\
0.86\end{array}$ & $\begin{array}{l}4.63 \\
104\end{array}$ \\
\hline $\begin{array}{l}57 \\
58\end{array}$ & $\frac{4}{4}$ & $\begin{array}{r}9.4 \\
9.4\end{array}$ & $\begin{array}{l}10 \\
10\end{array}$ & $\begin{array}{c}0 \\
30\end{array}$ & $\begin{array}{l}3 \\
4\end{array}$ & $\begin{array}{l}11.83 \\
12.81\end{array}$ & $\begin{array}{l}0.86 \\
0.80\end{array}$ & $\begin{array}{l}1.04 \\
4.53\end{array}$ \\
\hline 59 & $\frac{4}{4}$ & $\frac{9.4}{9.4}$ & $\frac{10}{10}$ & $\frac{50}{60}$ & $\frac{4}{2}$ & $\frac{12.01}{5.91}$ & $\frac{0.00}{1.53}$ & $\frac{4.05}{5.39}$ \\
\hline 60 & 4 & 9.4 & 10 & 90 & 2 & 5.65 & $\frac{1.53}{0.70}$ & 8.65 \\
\hline 61 & 4 & 9.4 & 10 & 120 & 2 & 2.4 & 4.98 & 11.14 \\
\hline 62 & 4 & 9.4 & 10 & 150 & 1 & 3.52 & 0.64 & 3.22 \\
\hline 63 & 4 & 9.4 & 10 & 180 & 2 & 9.48 & 0.67 & 4.95 \\
\hline$\frac{64}{65}$ & 5 & $\begin{array}{l}13.2 \\
132\end{array}$ & $\frac{6}{6}$ & 0 & 10 & 47.18 & 0.58 & $\frac{2.41}{2.86}$ \\
\hline 66 & 5 & $\frac{1.2}{13.2}$ & $\frac{0}{6}$ & $\frac{50}{60}$ & $\frac{1}{12}$ & $\frac{11.01}{75.02}$ & $\frac{0.37}{1.13}$ & $\begin{array}{l}2.86 \\
2.87\end{array}$ \\
\hline 67 & 5 & $\frac{13.2}{13.2}$ & $\frac{0}{6}$ & 90 & 10 & 33.25 & 0.65 & 9.62 \\
\hline 68 & 5 & 13.2 & 6 & 120 & 2 & 5.32 & 1.29 & 7.93 \\
\hline 69 & 5 & 13.2 & 6 & 150 & $\overline{2}$ & 8.6 & 0.69 & 3.23 \\
\hline 70 & 5 & 13.2 & 6 & 180 & 1 & 6.38 & 0.12 & 5.40 \\
\hline $\begin{array}{l}71 \\
72\end{array}$ & 5 & $\begin{array}{l}13.2 \\
132\end{array}$ & 8 & 0 & 13 & 69.83 & 1.22 & 1.10 \\
\hline 72 & $\frac{5}{5}$ & $\begin{array}{l}13.2 \\
132\end{array}$ & $\frac{8}{8}$ & $\begin{array}{r}30 \\
60\end{array}$ & $\frac{5}{3}$ & $\begin{array}{l}73.47 \\
3365\end{array}$ & 0.98 & 2.29 \\
\hline 74 & 5 & $\begin{array}{l}13.2 \\
13.2\end{array}$ & 8 & 90 & 9 & 39.57 & 0.81 & 6.79 \\
\hline 75 & 5 & 13.2 & 8 & 120 & 1 & 3.9 & 0.53 & 4.45 \\
\hline 76 & 5 & 13.2 & 8 & 150 & 2 & 6.35 & 0.33 & 4.92 \\
\hline 77 & 5 & 13.2 & 8 & 180 & 3 & 13.54 & 0.57 & 2.69 \\
\hline $\begin{array}{l}78 \\
79\end{array}$ & $\begin{array}{l}5 \\
5\end{array}$ & $\begin{array}{l}13.2 \\
13.2\end{array}$ & $\begin{array}{l}10 \\
10\end{array}$ & $\begin{array}{l}0 \\
30 \\
\end{array}$ & $\frac{10}{6}$ & $\frac{119.99}{91.64}$ & & \\
\hline & & 13.2 & 10 & 60 & 3 & 41.32 & 0. & \\
\hline 81 & 5 & 13.2 & 10 & 90 & 6 & 27.41 & 1.06 & 3.62 \\
\hline $\begin{array}{l}82 \\
83\end{array}$ & $\frac{5}{5}$ & $\begin{array}{l}13.2 \\
13.2\end{array}$ & $\frac{10}{10}$ & $\frac{120}{150}$ & $\frac{2}{3}$ & $\frac{4.69}{12.69}$ & 0. & $\begin{array}{l}1.92 \\
3.87\end{array}$ \\
\hline 84 & 5 & $\begin{array}{l}13.2 \\
13.2\end{array}$ & $\frac{10}{10}$ & 180 & $\frac{3}{1}$ & $\begin{array}{r}12.69 \\
4.46\end{array}$ & $\begin{array}{l}0.00 \\
0.19\end{array}$ & $\begin{array}{l}3.81 \\
7.76\end{array}$ \\
\hline 85 & 6 & 15.3 & 6 & 0 & 3 & 10.5 & & \\
\hline $\begin{array}{l}86 \\
87\end{array}$ & 6 & 15.3 & 6 & 30 & 9 & 64.27 & 1.98 & 5.45 \\
\hline $\begin{array}{l}87 \\
88\end{array}$ & 6 & $\begin{array}{r}15.3 \\
153\end{array}$ & 6 & 60 & 7 & $\begin{array}{r}27.53 \\
53.83\end{array}$ & 0.79 & $\begin{array}{l}1.53 \\
\end{array}$ \\
\hline 88 & $\frac{6}{6}$ & $\begin{array}{l}15.3 \\
15.3\end{array}$ & $\frac{6}{6}$ & $\begin{array}{l}90 \\
120\end{array}$ & $\frac{13}{2}$ & $\begin{array}{r}53.86 \\
3.75\end{array}$ & 0.83 & $\begin{array}{l}5.06 \\
4.74\end{array}$ \\
\hline 90 & 6 & & 6 & -7 & & 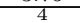 & & \\
\hline 91 & 6 & 15.3 & 6 & 180 & 1 & 8.97 & 0.74 & 3.80 \\
\hline 92 & 6 & 15.3 & & 0 & 10 & 25.33 & 0.75 & 1.71 \\
\hline 93 & 6 & 15.3 & 8 & 30 & 13 & 91.6 & 1.09 & 5.8 \\
\hline 94 & 6 & $\begin{array}{l}15.3 \\
\end{array}$ & 8 & 60 & 3 & 10.44 & 0.45 & 5.16 \\
\hline 95 & 6 & $\frac{1.5}{15.3}$ & $\frac{8}{8}$ & $\frac{90}{120}$ & $\frac{10}{1}$ & $\frac{47.48}{8.66}$ & $\frac{1.11}{0.58}$ & $\frac{8.56}{2.86}$ \\
\hline 97 & & & 8 & 1 & & 5 & 0 & 3 \\
\hline 98 & 6 & 15.3 & 8 & 180 & 1 & 5.78 & 0.15 & 4.84 \\
\hline 99 & 6 & 15.3 & 10 & 0 & 11 & 71.98 & 0.70 & 1.48 \\
\hline 100 & 6 & 15.3 & 10 & 30 & 5 & 53.67 & 1.00 & 5.47 \\
\hline 101 & 6 & 15.3 & 10 & 60 & 8 & 49.1 & & \\
\hline $\begin{array}{l}102 \\
103\end{array}$ & 6 & $\begin{array}{l}15.3 \\
15.3\end{array}$ & $\frac{10}{10}$ & $\begin{array}{l}90 \\
120\end{array}$ & $\frac{11}{1}$ & $\begin{array}{l}55.37 \\
3.59\end{array}$ & $\begin{array}{l}1.32 \\
0.79\end{array}$ & $\begin{array}{r}2.99 \\
7.09\end{array}$ \\
\hline 104 & 6 & 15.3 & 10 & 150 & 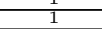 & 4.21 & 0.52 & 6.14 \\
\hline 105 & 6 & 15.3 & 10 & 180 & 1 & 4.78 & 0.02 & $\frac{0.14}{4.83}$ \\
\hline
\end{tabular}


Table B.3: SPA+AHC $5 \mathrm{~m}$ low hover full results.

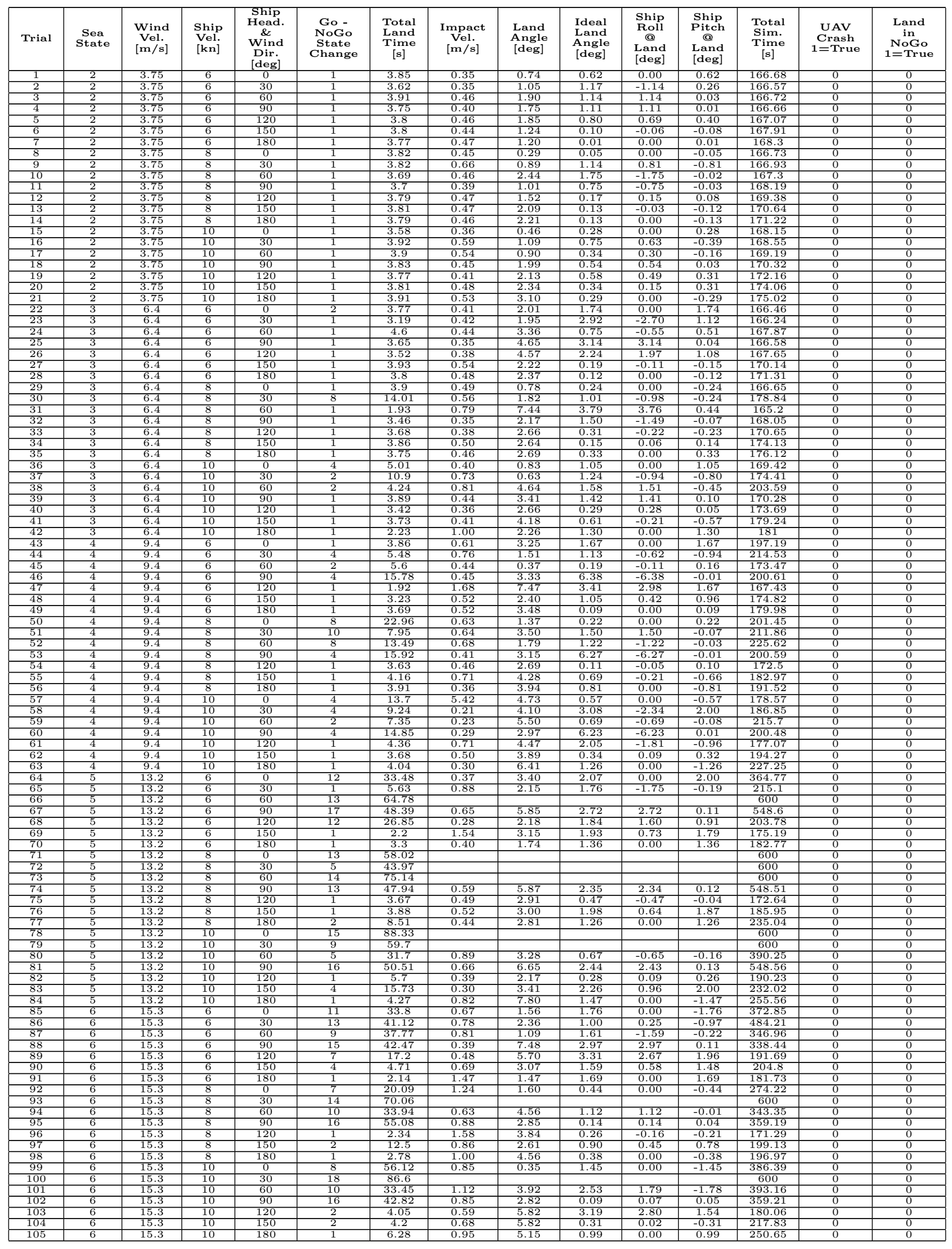


Table B.4: LPI $5 \mathrm{~m}$ low hover full results.

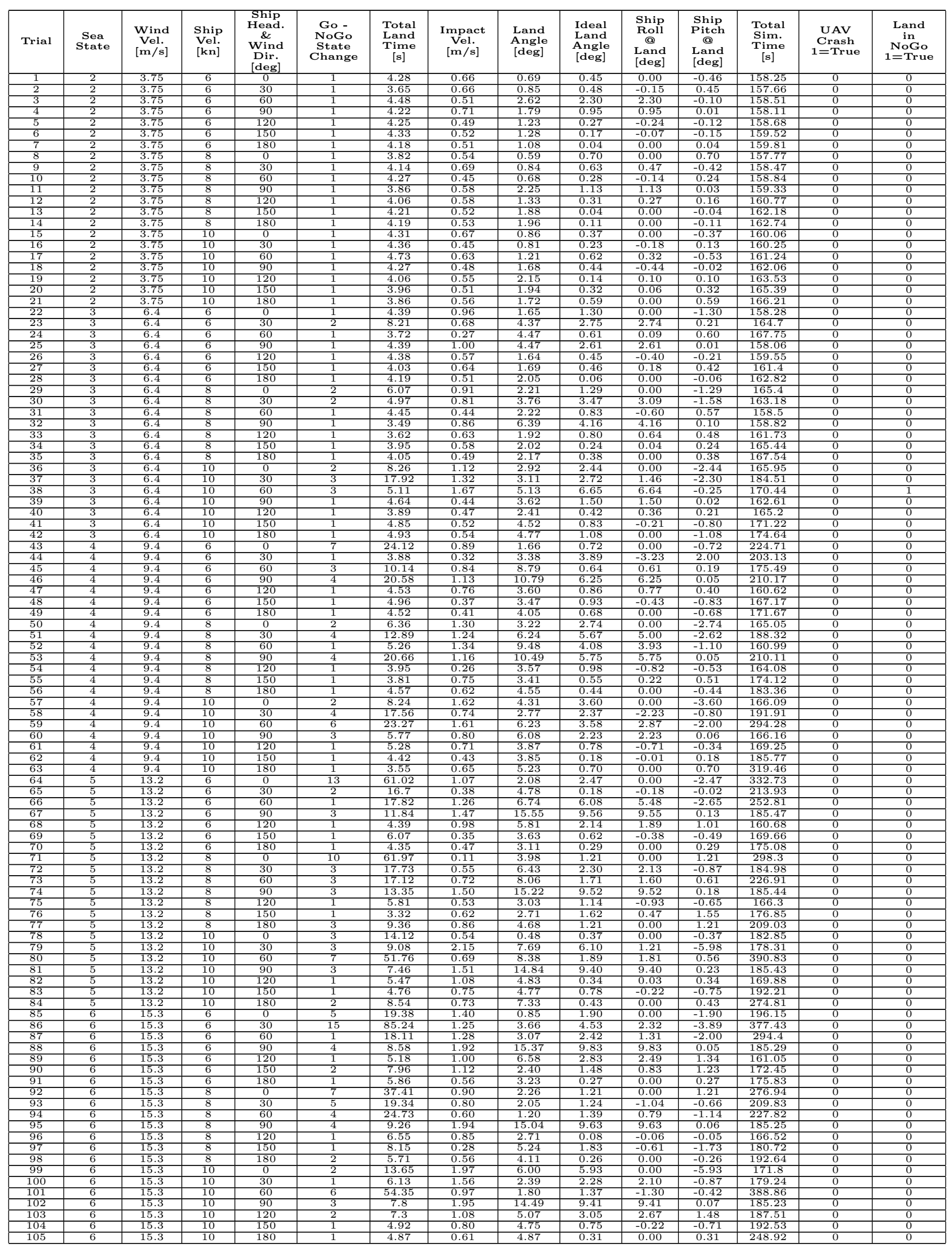


Table B.5: LPI+SPA 5m low hover full results.

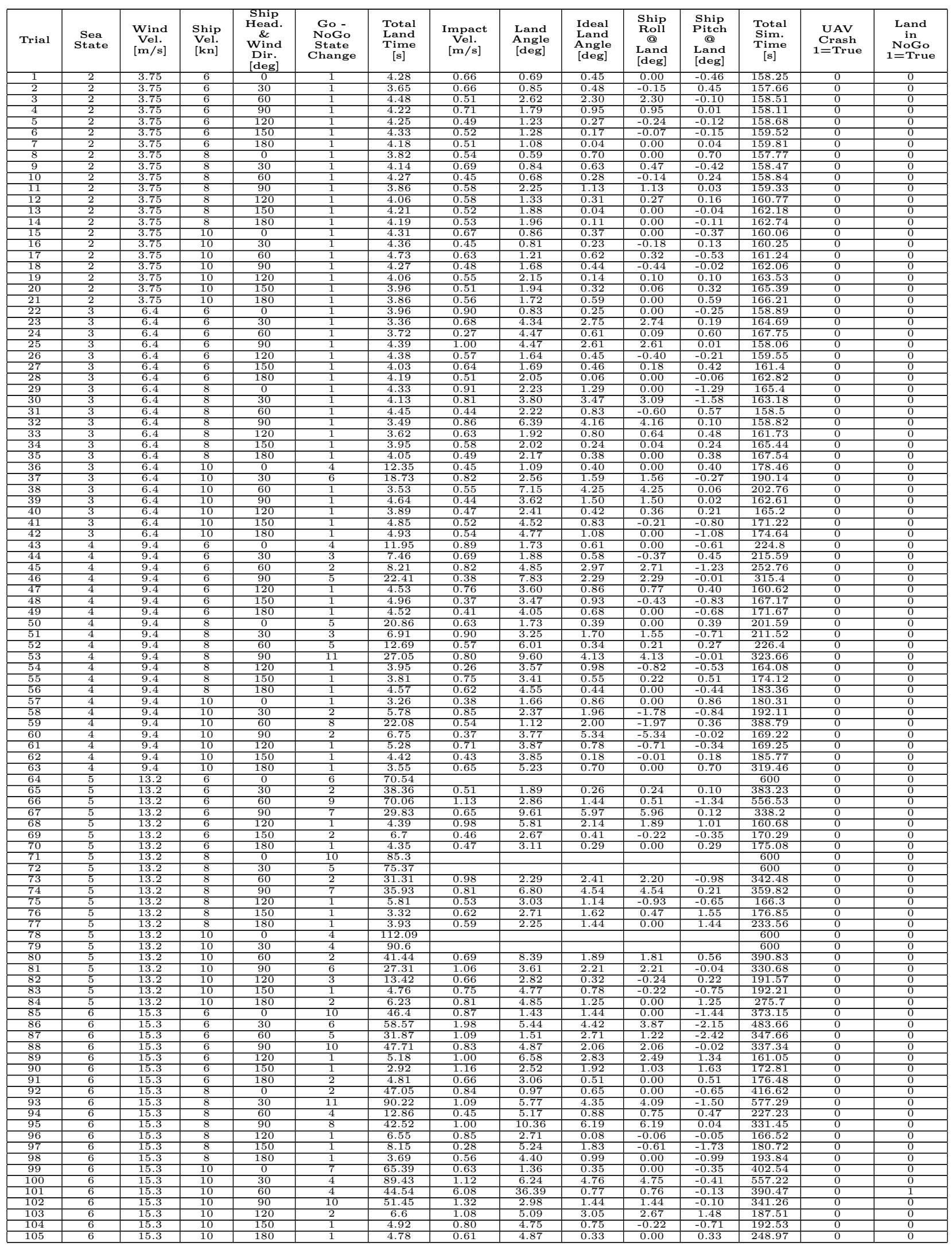


Table B.6: LPI+SPA+AHC 5m low hover full results.

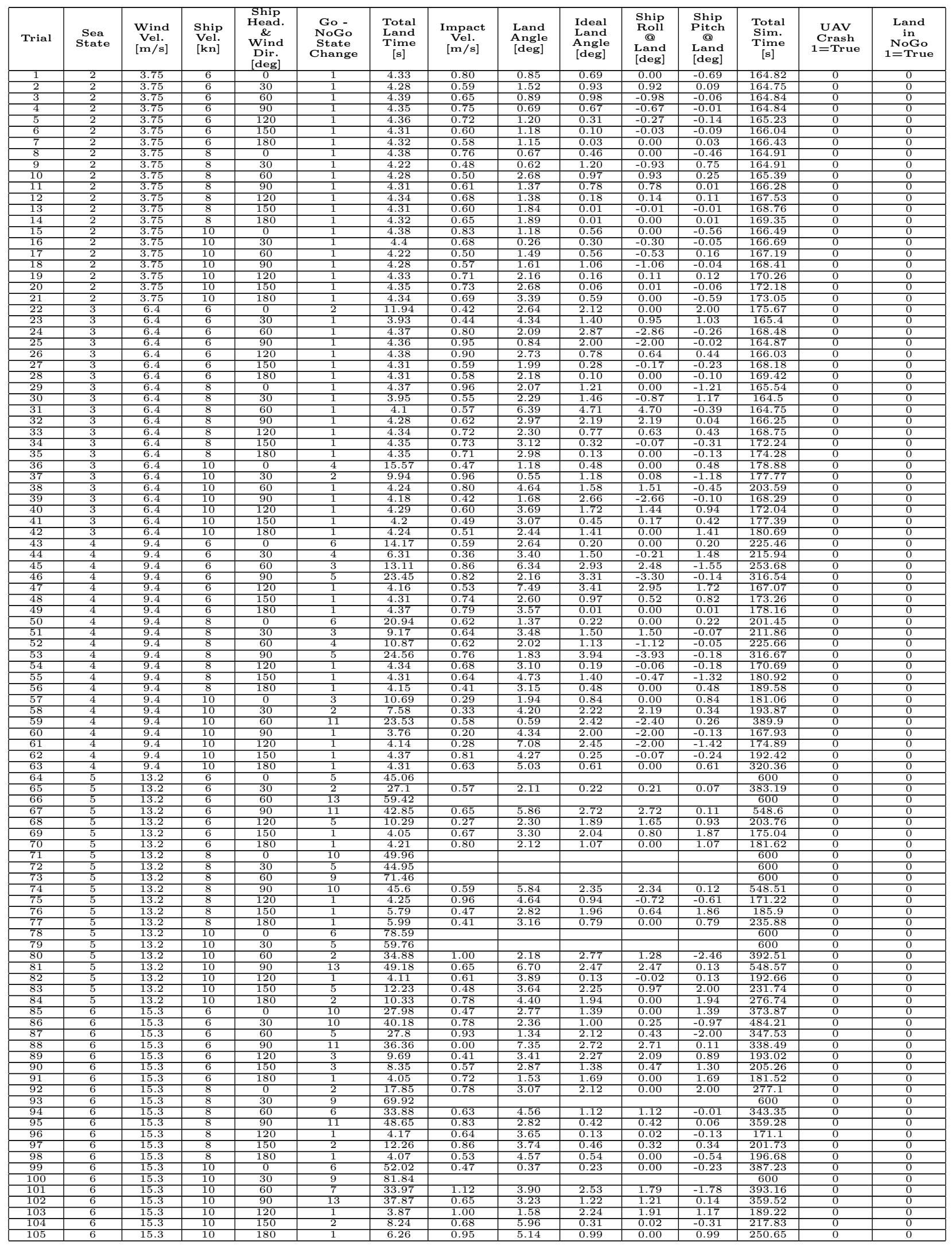


Table B.7: LPI+AHC 5m low hover full results.

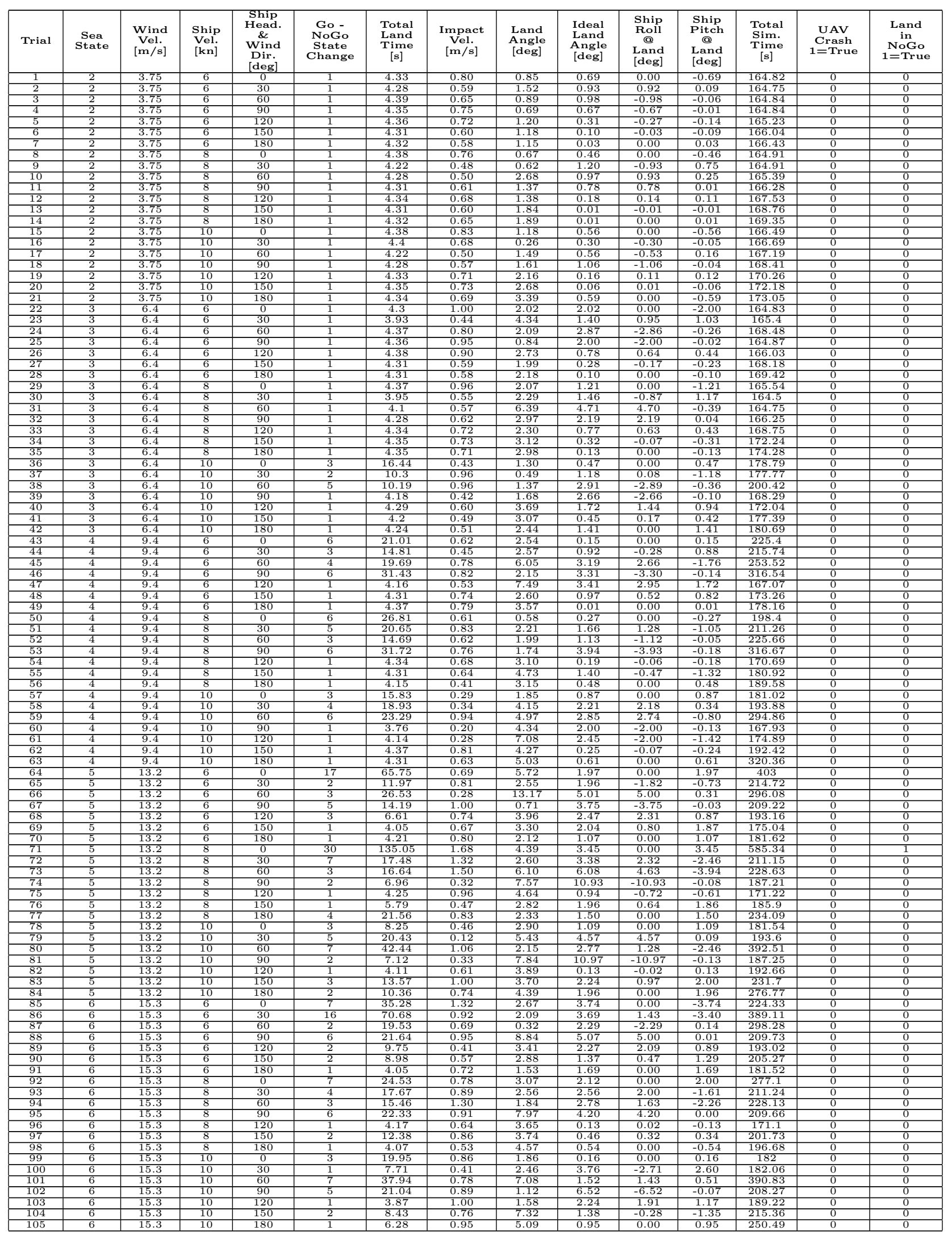

